\title{
Modeling and Simulation of a Free-Piston Engine with Electrical Generator Using $\mathrm{HCCl}$ Combustion
}

\author{
Mohammad Alrbai
}

Follow this and additional works at: https://researchrepository.wvu.edu/etd

\section{Recommended Citation}

Alrbai, Mohammad, "Modeling and Simulation of a Free-Piston Engine with Electrical Generator Using HCCI Combustion" (2016). Graduate Theses, Dissertations, and Problem Reports. 5086.

https://researchrepository.wvu.edu/etd/5086

This Dissertation is protected by copyright and/or related rights. It has been brought to you by the The Research Repository @ WVU with permission from the rights-holder(s). You are free to use this Dissertation in any way that is permitted by the copyright and related rights legislation that applies to your use. For other uses you must obtain permission from the rights-holder(s) directly, unless additional rights are indicated by a Creative Commons license in the record and/ or on the work itself. This Dissertation has been accepted for inclusion in WVU Graduate Theses, Dissertations, and Problem Reports collection by an authorized administrator of The Research Repository @ WVU.

For more information, please contact researchrepository@mail.wvu.edu. 


\title{
Modeling and Simulation of a Free-Piston Engine with Electrical Generator Using $\mathrm{HCCl}$ Combustion
}

\author{
Mohammad Alrbai \\ Dissertation Submitted to the
}

Benjamin M. Statler College of Engineering and Mineral Resources at West

Virginia University

in partial fulfillment of the requirements for the degree of

Doctorate of Philosophy in Mechanical Engineering

Nigel Clark, Ph.D., Chair

Hailin Li, Ph.D.

Cosmin Dumitrescu, Ph.D.

Terence Musho, Ph.D.

Fernando V. Lima, Ph.D.

Department of Mechanical and Aerospace Engineering

Morgantown, West Virginia

2016

Keywords: Free Piston Engine, Electrical Generator, Chemical kinetics, $\mathrm{HCCl}$ Combustion, Model Control, EGR, Equivalence Ratio.

Copyright 2016 Mohammad Alrbai 


\section{ABSTRACT}

\section{Modeling and Simulation of a Free-Piston Engine with Electrical Generator using $\mathrm{HCCl}$ Combustion}

\section{Mohammad Alrbai}

Free-piston engines have the potential to challenge the conventional crankshaft engines by their design simplicity and higher operational efficiency. Many studies have been performed to overcome the limitations of the free-piston devices especially the stability and control issues

The investigations within the presented dissertation aim to satisfy many objectives by employing the approach of chemical kinetics to present the combustion process in the free-piston engine. This approach in addition to its advanced accuracy over the empirical methods, it has many other features like the ability to analyze the engine emissions. The effect of the heat release rate (HRR) on the engine performance is considered as the main objective. Understanding the relation between the HRR and the piston dynamics helps in enhancing the system efficiency and identifying the parameters that affect the overall performance. The dissertation covers some other objectives that belongs to the combustion phasing. Exhaust gas recirculation (EGR), equivalence ratio and the intake temperature represent the main combustion parameters, which have been discussed in this dissertation. To obtain the stability in system performance, the model requires a proper controller to simulate the operation and manage the different system parameters; for this purpose, different controlling techniques have been employed. In addition, the dissertation considers some other topics like engine emissions, fuels and fuels mechanisms.

The model of the study describes the processes within a single cylinder, two stroke engine, which includes springs to support higher frequencies, reduce cyclic variations and sustain the engine compression ratio. An electrical generator presents the engine load; the generator supports different load profiles and play the key role in controlling the system. The $1^{\text {st }}$ law of thermodynamics and Newton's $2^{\text {nd }}$ law are applied to couple the piston dynamics with the engine thermodynamics. The model governing equations represent a single zone perfectly stirred reactor (PSR) which contain a perfect mixing ideal gas mixture. The chemical kinetics approach is applied using Cantera/ MATLAB ${ }^{\circ}$ toolbox, which presents the combustion process. In this research, a homogenous charge compression ignition ( $\mathrm{HCCl}$ ) at different operational conditions is used. $\mathrm{HCCl}$ engines have high efficiencies and low emissions and can work within a wide range of fuels.

The results have been presented in a multi-cycle simulation and a parametric study forms. In the case of the multi-cycle simulation, a 100 cycles of the engine operation have been simulated. The overall work that is delivered to the electrical generator presents $47 \%$ of the total fuel energy. The model indicates an average frequency of $125 \mathrm{H}_{\mathrm{z}}$ along the operational cycles. In order to eliminate the cyclic variations and ensure a continuous operation, a proportional derivative (PD) controller has been employed. The controller adjusts the generator load in order to minimize the difference between the bottom dead center (BDC) locations along the operation cycles. The PD controller shows weakness in achieving the full steady state operation, for this purpose; a proportional integral (PI) controller has been implemented. The PI controller seeks to achieve a specific compression ratio. The results show that; the PI controller indicates 
unique behavior after 15 cycles of operation where the model ended to fluctuate between two compression ratios only. The complex relation between the thermodynamics and the dynamics of the engine is the greatest challenge in examining the effectiveness of the PI controller.

In the parametric investigations, EGR examinations show that $\mathrm{NO}_{\mathrm{x}}$ emission is reduced to less than the half, as $30 \%$ of EGR is used; this occurs due to the EGR thermal and dilution effects, which cause significant drop in the peak bulk temperature and CO emissions as well. Under the applied conditions, EGR has the ability to raise the work output ratio by increasing the engine compression ratio. The examination of the EGR temperature on the engine performance indicates that cooled EGR charges have the advantage over the hot EGR mixtures on enhancing the work output ratio. At the same time, EGR temperature affects the NOx formation by speeding its instantaneous reactions rate. The dissertation includes a study of the effect of the intake temperature and the equivalence ratio $(\phi)$ as well. The increasing in the intake temperature reduce the time needed for ignition, but leads to a reduction in the work output ratio at the same time. Such results can help in studying high knock resistance fuels where ignition delay is a matter. In the case of the equivalence ratio, lean mixtures show efficiencies that exceed $50 \%$ compared to those at the stoichiometric conditions. In the case of the ultra-lean $(\phi<0.5)$ combustion, the results show that the $\mathrm{NO}_{\mathrm{x}}$ emission is with the minimal levels as well as the $\mathrm{CO}$ and the unburned hydrocarbons (UHC) emissions.

Sensitivity analysis to the chemical kinetic mechanism for the fuel combustion has been presented also in the dissertation. Many mechanisms for different fuels have been investigated, for example; a modified mechanism for Methane that includes 36 species and 222 reactions has been compared with the full GRI 3.0 mechanism ( 53 species and 325 reactions). The results of this comparison indicate that the modified mechanism has the potential to replace the full one in some cases like in demonstrating the engine operation, but not in the engine emissions analysis. 


\section{Dedication}

I dedicate this work to my parents and to all of my family members. 


\section{Acknowledgments}

First, I would like to thank Allah for his guidance and care during my whole life. I could not have met the challenges of this research without him.

All of the appreciations and thanks go to my advisor Dr. Nigel Clark and my Co. Advisor Dr. Hailin Li. In addition, I want to thank all of the other committee members, Dr. Terence Musho, Dr. Cosmin Dumitrescu, and Dr. Fernando V. Lima. Their help and Advices were the motivators of my work.

Special thanks goes to Dr. Hany Ammar for his continuous support along my study. At the same time, many thanks go to George Berry Chair for the financial support during this research.

Finally, thanks to all of those friends, colleagues, instructors, and mentors who gave some of their time to make this work successful. 


\section{Table of Contents}

Dedication.

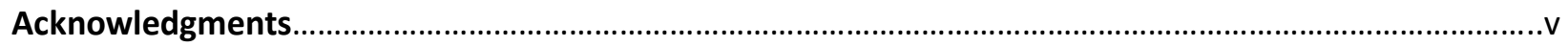

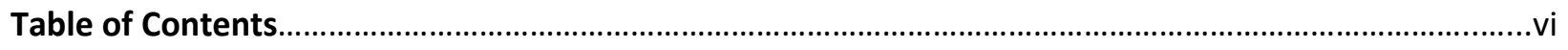

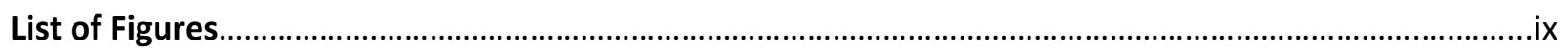

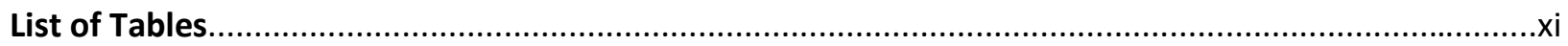

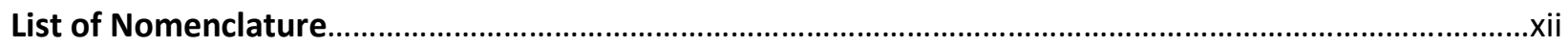

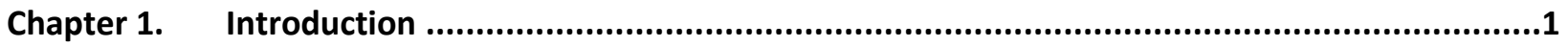

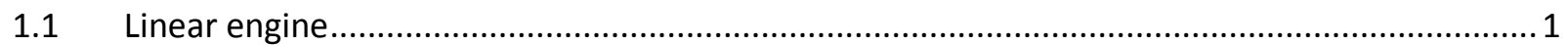

$1.2 \quad$ Homogeneous charge compression ignition engines .......................................................... 1

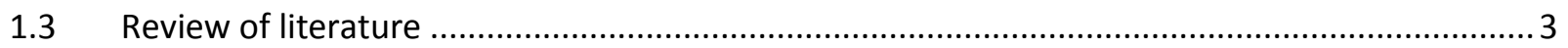

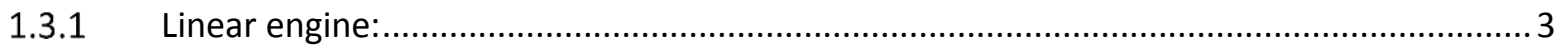

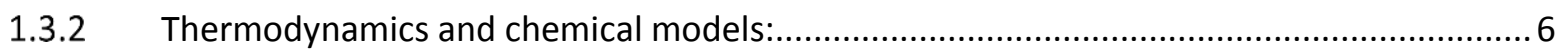

1.3.3 Thermo-chemical models used in free-piston engines....................................................... 8

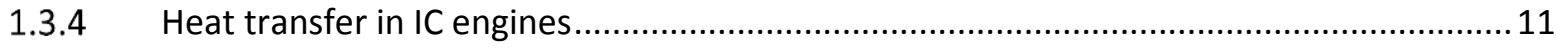

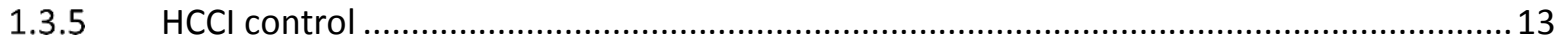

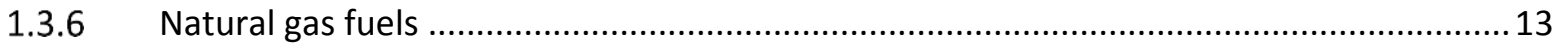

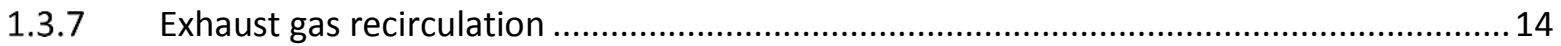

1.3.8 Intake temperature effect on engine performance .................................................... 15

1.3.9 The equivalence ratio effect on engine performance ................................................. 16

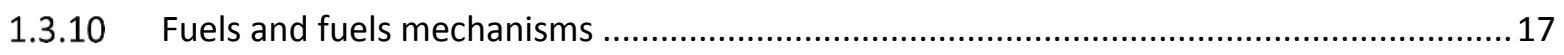

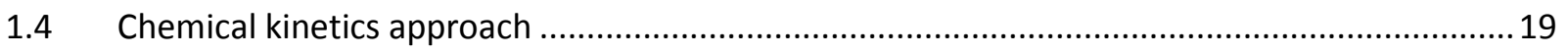

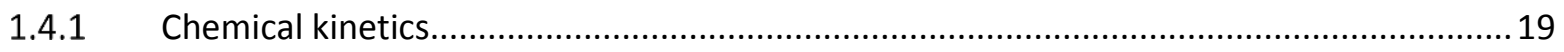

1.4.2 Species governing equations:

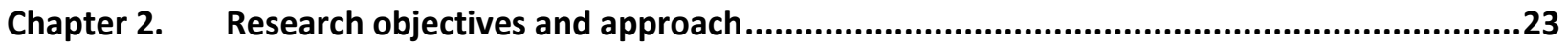

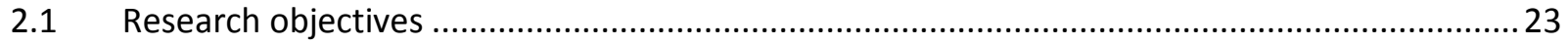

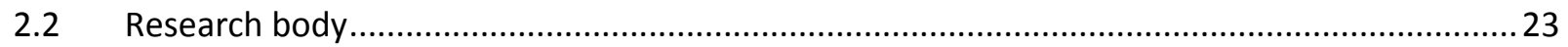

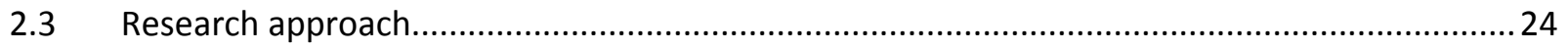

Chapter 3. Free-piston and chemical kinetics approach..........................................................26

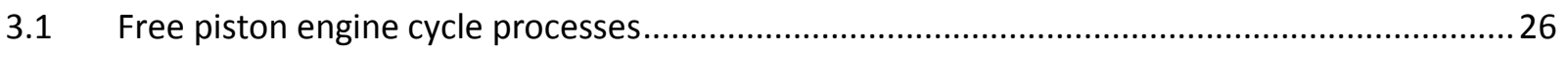

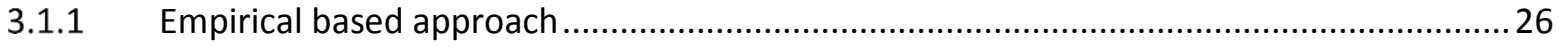

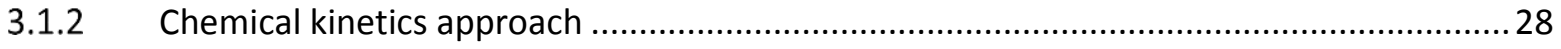




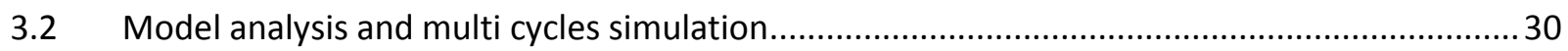

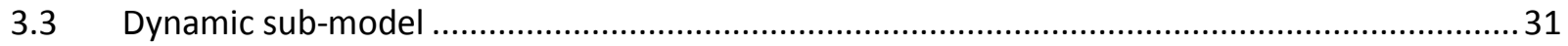

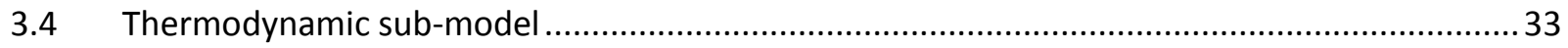

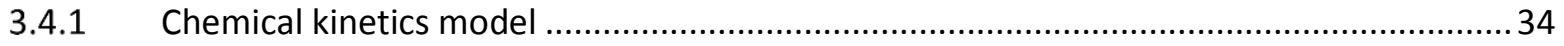

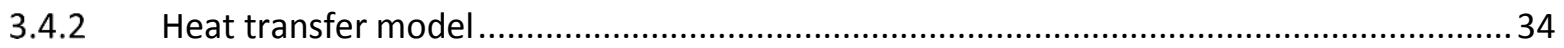

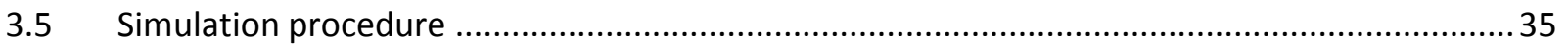

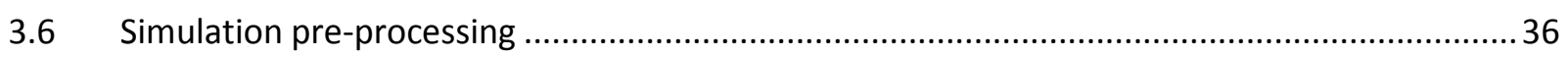

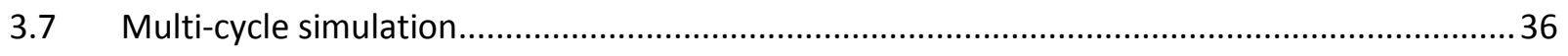

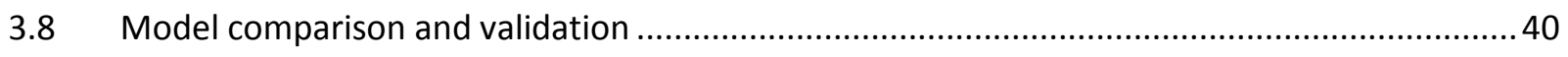

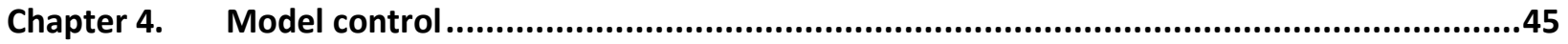

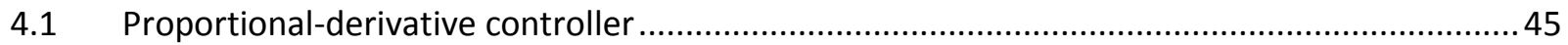

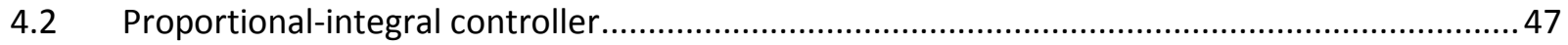

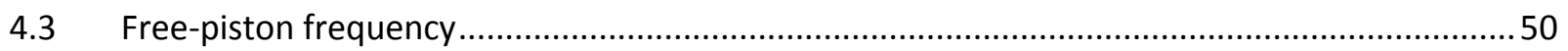

Chapter 5. Exhaust gas recirculation (EGR) impact on free-piston engine.................................52

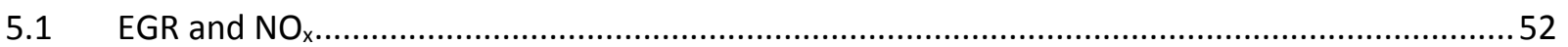

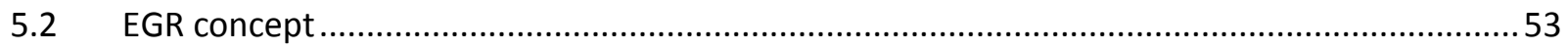

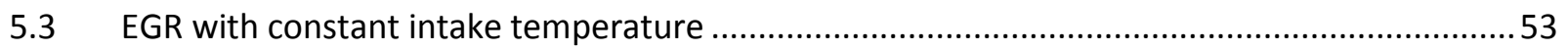

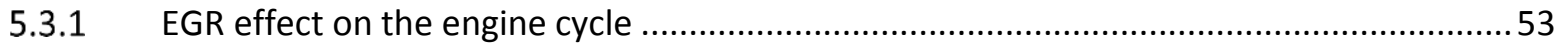

5.3.2 EGR effects on free-piston engine emissions. ............................................................ 60

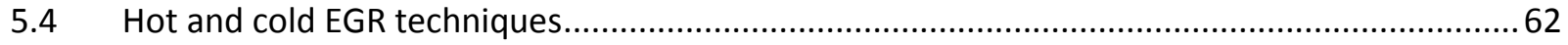

Chapter 6. Intake temperature and equivalence ratio effects....................................................68

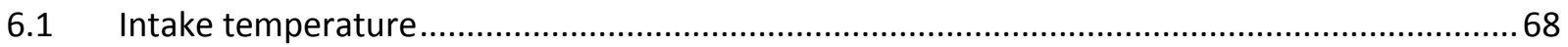

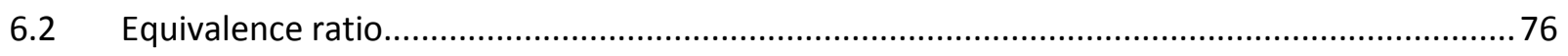

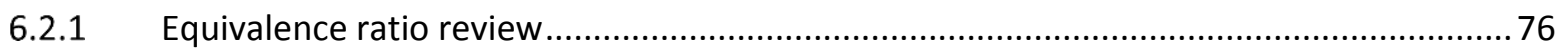

6.2.2 Variable equivalence ratio at constant intake temperature: .......................................... 77

6.2.3 Equivalence ratio effect at variable intake temperature.................................................. 88

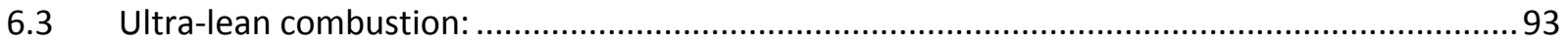

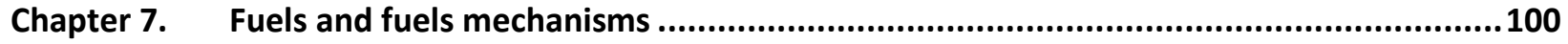

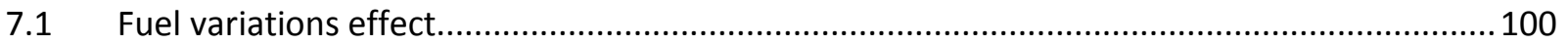

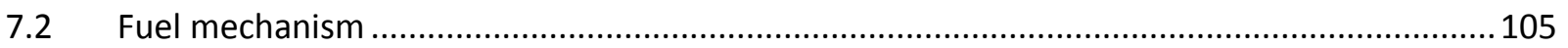

Chapter 8. Summary and conclusions ....................................................................................111

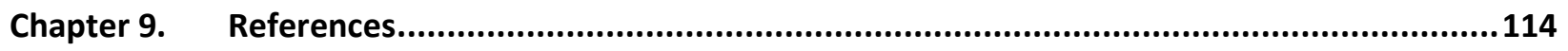

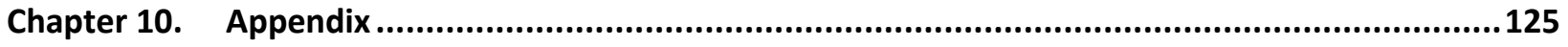




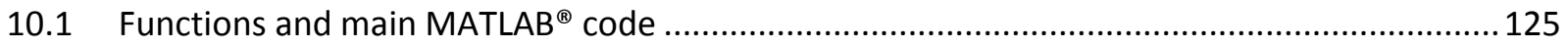

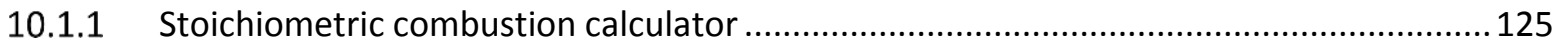

10.1.2 Piston position, velocity and acceleration calculation ............................................... 126

10.1.3 Main MATLAB ${ }^{\circledR}$ code for multi-cycle calculations ….................................................... 128

10.2 Modified version of GRI 3.0 mechanism (species reactions) ...........................................136 


\section{List of Figures}

Figure 1 Sandia's Free Piston Engine - Linear Alternator Prototype [21] ….......................................... 4

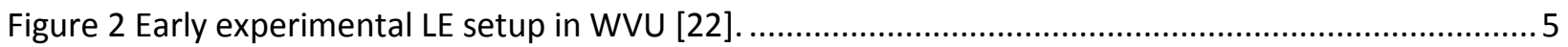

Figure 3 Device schematic of Newcastle University's free piston engine [27]. ......................................... 5

Figure 4 Associated input and output files for the chemical kinetic model [51] .................................... 10

Figure 5 Interaction between chemical kinetics and gas dynamics [52] ............................................. 11

Figure 6 Schematic Representation of a Well Mixed Reactor Module [110] . .........................................2 21

Figure 7 In-Cylinder Pressure vs. Piston Assembly Displacement [23] ..................................................2

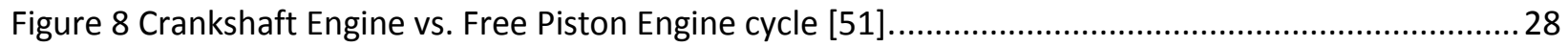

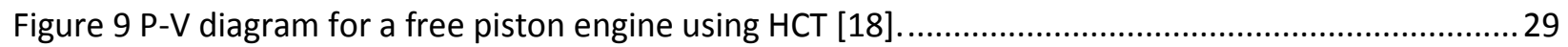

Figure 10 Cycle pressure including the combustion event using HCT [18] ............................................29

Figure 11 (a) Free piston engine schematic, (b) Dynamic and thermodynamic model diagram................31

Figure 12 Effect of load profile of the piston dynamics [41] ................................................................ 33

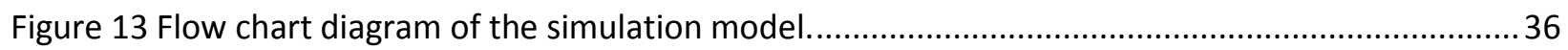

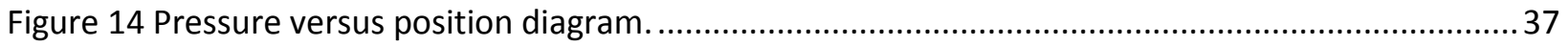

Figure 15 Position, velocity, and acceleration versus time for the first 0.2 seconds of simulated

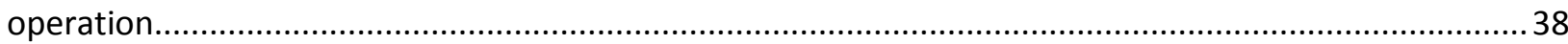

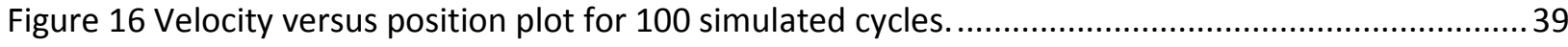

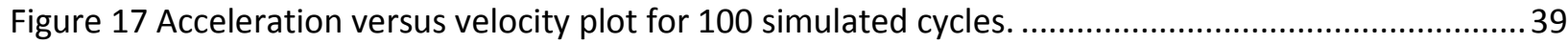

Figure 18 The first four cycles of pressure and temperature versus time.................................................40

Figure 19 In-cylinder pressure for Cantera and Chemkin ${ }^{\circledR}$ models. .......................................................42

Figure 20 In-cylinder temperature for Cantera and Chemkin ${ }^{\circledR}$ models............................................... 42

Figure 21 Pressure-volume diagram for Cantera and Chemkin models..............................................43

Figure 22 Single cycle comparison between current model with Cantera and the SNL model. ................ 44

Figure 23 Proportional- derivative (PD) Controller diagram used in the simulation............................. 45

Figure 24 Cycle to cycle variations of piston mean velocity over 100 cycles for two controllers. .............46

Figure 25 Cycle to cycle variations of cylinder compression ratio over 100 cycles for two controllers..... 47

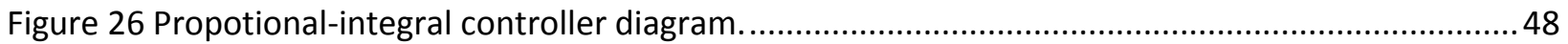

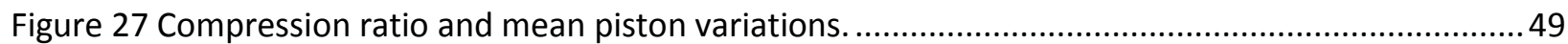

Figure 28 Piston position profile for the PI contoller......................................................................... 49

Figure 29 Free-piston engine frequency under different piston mass. .................................................50

Figure 30 Free-piston engine frequency at different spring stuffiness. ...............................................51

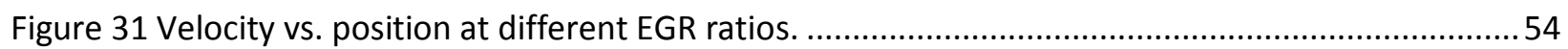

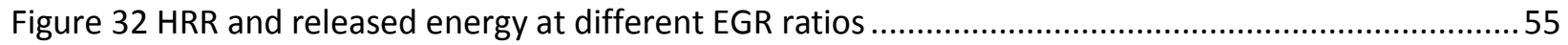

Figure 33 Temperature-time diagram at different EGR ratios. ........................................................5

Figure 34 In-cylinder pressure variations at different EGR ratios..........................................................5

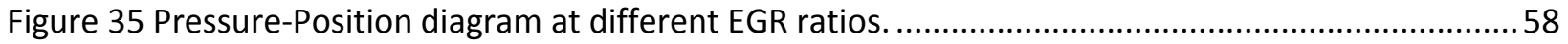

Figure 36 Temerature-Position diagram at different EGR ratios......................................................58

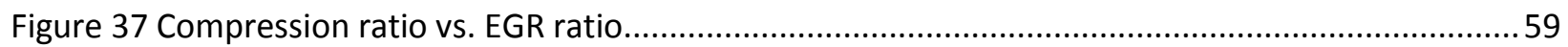

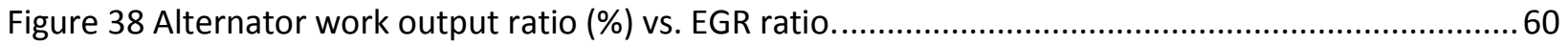

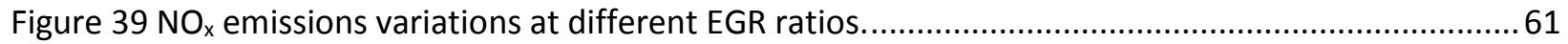

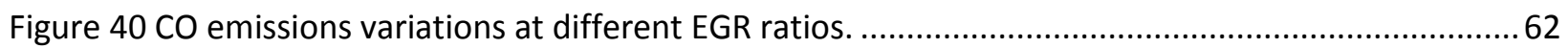

Figure 41 Peak pressure variations at different intake temperature.....................................................63 
Figure 42 Compression ratio variations at different intake temperature.

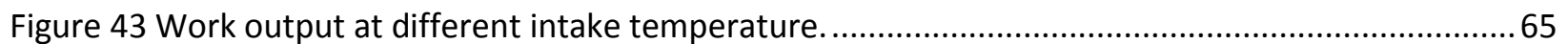

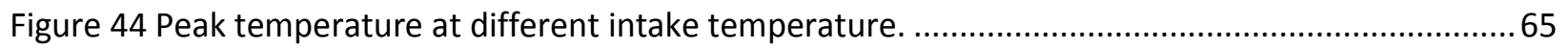

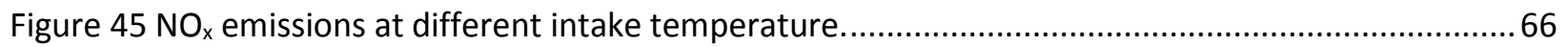

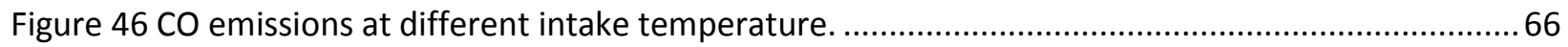

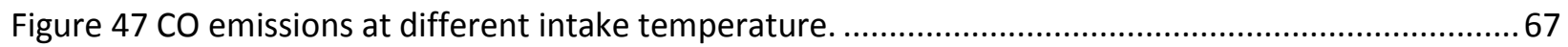

Figure 48 Velocity vs. position at different intake temperature. .........................................................69

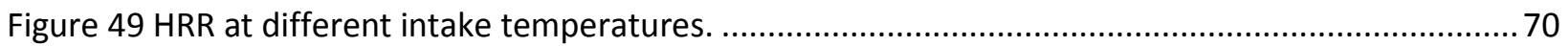

Figure 50 In-cylinder pressure at different intake temperature............................................................. 71

Figure 51 Compression ratio at different intake temperature. ............................................................. 71

Figure 52 In-cylinder pressure-position diagram at different intake temperature................................. 72

Figure $53 \mathrm{ln}$-cylinder Temperature-position diagram at different intake temperature............................. 73

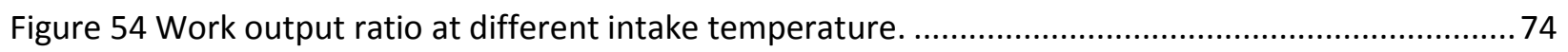

Figure 55 In-cylinder temperature at different intake temperature.................................................. 74

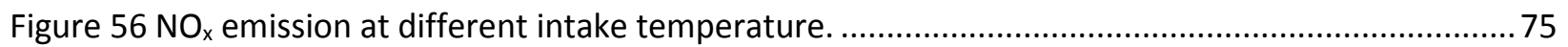

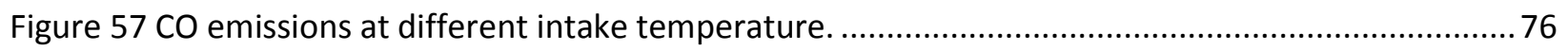

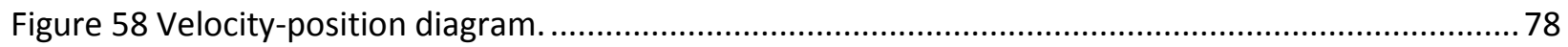

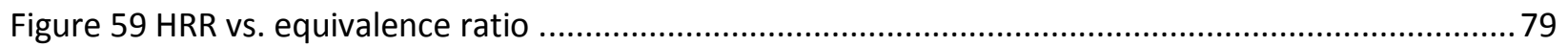

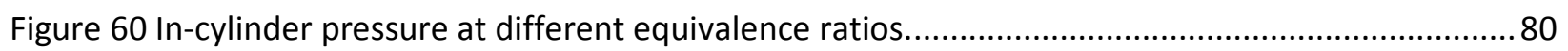

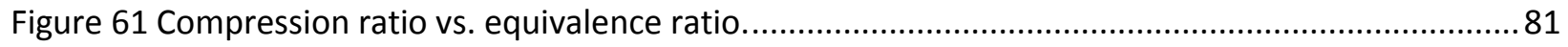

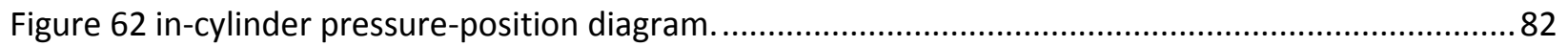

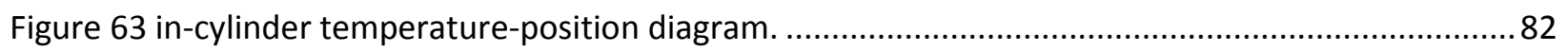

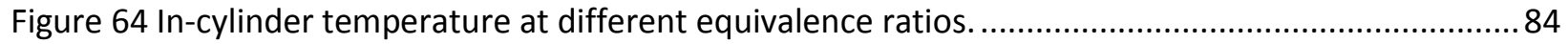

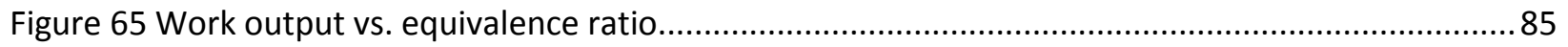

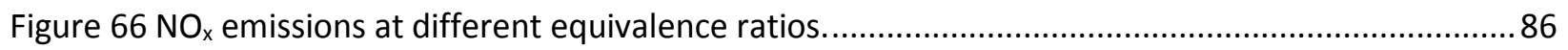

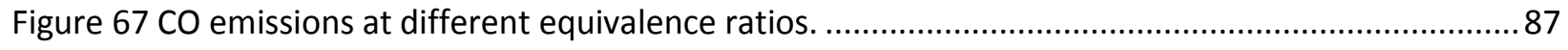

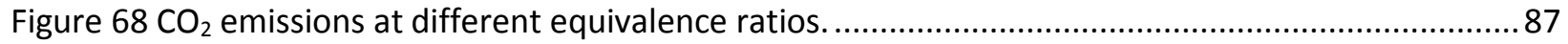

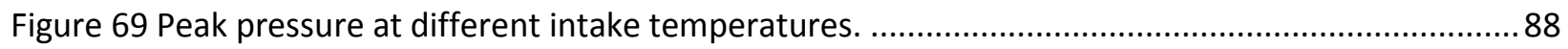

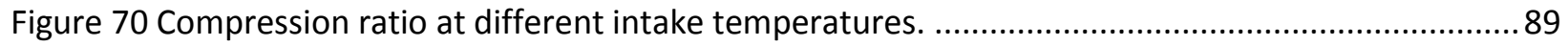

Figure 71 Work output ratio at different intake temperatures..........................................................90

Figure 72 In-cylinder peak temperature at different intake temperature. .............................................91

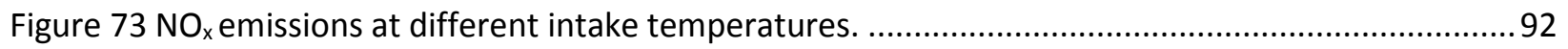

Figure 74 NOx variations with the maximum peak temperature........................................................ 92

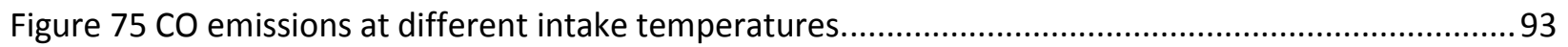

Figure 76 HRR at different equivalence ratios (ultra-lean)................................................................ 94

Figure 77 In-cylinder pressure at different equivalence ratios (ultra-lean)............................................95

Figure 78 Pressure-position diagram at different equivalence ratios (ultra-lean) ..................................96

Figure 79 Compression ratio at different equivalence ratios (ultra-lean)............................................. 96

Figure 80 Work output ratio vs. equivalence ratio (ultra-lean)........................................................97

Figure 81 In-cylinder temperature at different equivalence ratios (ultra-lean)......................................98

Figure $82 \mathrm{NO}_{x}$ emissions at different equivalence ratios (ultra-lean) ..................................................... 99

Figure $83 \mathrm{CO}$ emissions at different equivalence ratios (ultra-lean).................................................99

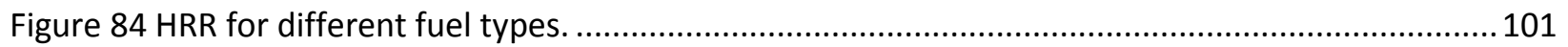

Figure 85 In-cylinder pressure for different fuels.......................................................................... 102 


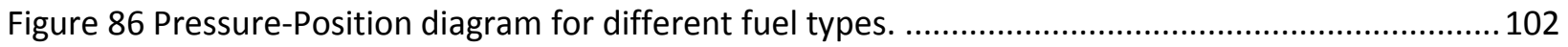

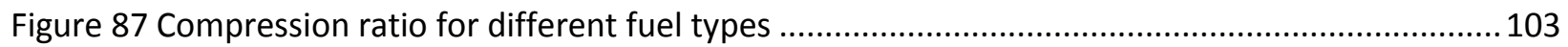

Figure 88 In-cylinder temperature for different fuel types. ................................................................ 104

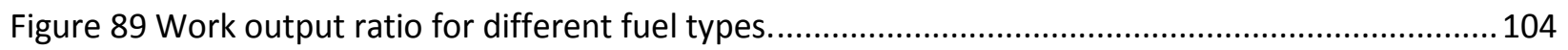

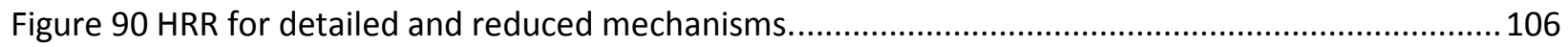

Figure 91 In-cylinder pressure (detailed vs. reduced mechanisms). .................................................... 107

Figure 92 Pressure-position diagram (detailed vs. reduced mechanisms) ............................................ 108

Figure 93 Compression ratio (detailed vs. reduced mechanism). ........................................................ 108

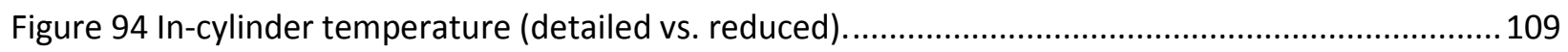

Figure 95 Work output ratio (detailed vs. reduced). ............................................................................. 110

\section{List of Tables}

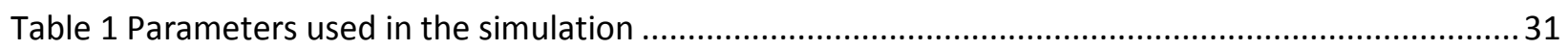

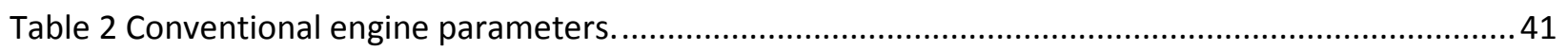

Table 3 Simulated SNL engine models specifications. ....................................................................... 44

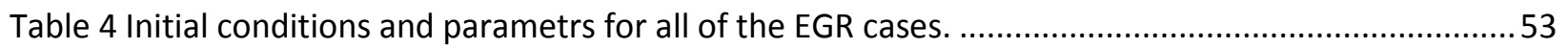

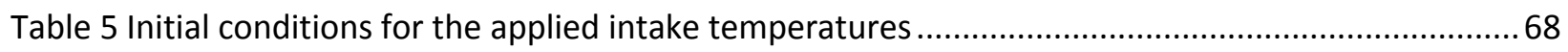

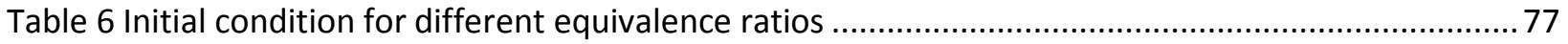

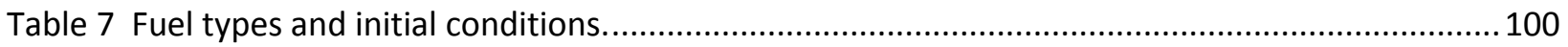

Table 8 Detailed and reduced mechanisms of some famous fuels. ...................................................... 105 


\section{List of Nomenclature}

\begin{tabular}{|c|c|}
\hline HCCI & $\begin{array}{l}\text { Homogeneous charge compression } \\
\text { ignition }\end{array}$ \\
\hline HCT & $\begin{array}{l}\text { Hydrodynamics, Chemistry, and } \\
\text { Transport }\end{array}$ \\
\hline TDC & Top dead center \\
\hline $\mathrm{BDC}$ & Bottom dead center \\
\hline $\mathrm{CR}$ & Compression ratio \\
\hline EGR & Exhaust gas recirculation \\
\hline $\mathrm{P}$ & Pressure $(\mathrm{Pa})$ \\
\hline $\mathrm{T}$ & Temperature (K) \\
\hline $\mathrm{T}_{\text {wall }}$ & Wall temperature $(\mathrm{K})$ \\
\hline $\operatorname{Re}$ & Reynolds’s number \\
\hline $\mathrm{Nu}$ & Nusselt`s number \\
\hline $\mathrm{U}$ & Internal energy $(\mathrm{kJ} \bullet \mathrm{kg}-1)$ \\
\hline $\mathrm{V}$ & Volume (m3) \\
\hline $\mathrm{m}$ & Piston mass $(\mathrm{kg})$ \\
\hline $\mathrm{R}$ & Gas constant $(\mathrm{J}-1 \cdot \mathrm{K} \cdot \mathrm{mol}-1)$ \\
\hline$\eta_{\mathrm{s}}$ & Scavenging Efficiency \\
\hline $\mathrm{Q}_{\mathrm{comb}}$ & Heat release rate $(\mathrm{kJ} \bullet \mathrm{s}-1)$ \\
\hline$Q_{\text {loss }}$ & Heat transfer rate $(\mathrm{kJ} \bullet \mathrm{s}-1)$ \\
\hline $\mathrm{h}$ & Heat transfer coefficient $(\mathrm{kJ} \bullet \mathrm{m}-2 \cdot \mathrm{K}-1)$ \\
\hline $\mathrm{h}_{(\mathrm{i}, \mathrm{m})}$ & Molar enthalpy $(\mathrm{kJ} \bullet \mathrm{kmol}-1)$ \\
\hline $\mathrm{i}$ & Specie number \\
\hline $\mathrm{N}$ & Total number of species \\
\hline $\mathrm{F}_{\mathrm{p}}$ & Pressure force $(\mathrm{N})$ \\
\hline $\mathrm{F}_{\mathrm{w}}$ & Alternator load $(\mathrm{N})$ \\
\hline$F_{f}$ & Friction force $(\mathrm{N})$ \\
\hline $\mathrm{F}_{\mathrm{e}}$ & Gravity force $(\mathrm{N})$ \\
\hline $\mathrm{F}_{\mathrm{s}}$ & Spring force $(\mathrm{N})$ \\
\hline $\mathrm{x}$ & Translator position (m) \\
\hline $\mathrm{v}$ & Translator velocity $(\mathrm{m} \bullet \mathrm{s}-1)$ \\
\hline $\mathrm{a}$ & Translator acceleration $(\mathrm{m} \bullet \mathrm{s}-2)$ \\
\hline $\mathrm{b}$ & Cylinder bore (mm) \\
\hline$S_{\max }$ & Maximum stroke length (mm) \\
\hline$d_{E P}$ & Distance to the exhaust port (mm) \\
\hline A & Piston head surface area (m2) \\
\hline $\mathrm{F}_{\mathrm{e}}$ & Gravity force $(\mathrm{N})$ \\
\hline $\mathrm{F}_{\mathrm{s}}$ & Spring force $(\mathrm{N})$ \\
\hline $\mathrm{V}_{\mathrm{P}}$ & Mean piston speed $(\mathrm{m} \bullet \mathrm{s}-1)$ \\
\hline $\mathrm{A}_{\text {wall }}$ & Heat loss area $(\mathrm{m} 2)$ \\
\hline$\omega_{\mathrm{i}}$ & Net production rate $(\mathrm{mol} \cdot \mathrm{L}-1 \cdot \mathrm{s}-1)$ \\
\hline $\mathrm{t}$ & Time $(s)$ \\
\hline $\mathrm{g}$ & Gravity acceleration $(\mathrm{m} \bullet \mathrm{s}-2)$ \\
\hline $\mathrm{f}_{\mathrm{mep}}$ & Friction mean effective pressure $(\mathrm{Pa})$ \\
\hline $\mathrm{A}_{\text {alt }}$ & Alternator load constant $(\mathrm{N})$ \\
\hline K & Spring stiffness $(\mathrm{N} \bullet \mathrm{m}-1)$ \\
\hline
\end{tabular}




\begin{tabular}{ll|}
\hline $\mathrm{C}_{\mathrm{A}}$ & Alternator coefficient \\
$\mathrm{W}_{\mathrm{A}}$ & Alternator weight \\
$\mathrm{PD}$ & Proportional-derivative \\
$\mathrm{PI}$ & Proportional-Integral \\
$\mathrm{CV}$ & Control volume \\
$\mathrm{CH}_{4}$ & Methane \\
$\mathrm{CO}_{2}$ & Carbon dioxide \\
$\mathrm{H}_{2}$ & Hydrogen \\
$\mathrm{SNL}$ & Sandia National Laboratories \\
WVU & West Virginia University \\
\hline
\end{tabular}




\section{Chapter 1. Introduction}

\subsection{Linear engine}

With increasing the energy demands in the world, searching for efficient, simple and durable engines is required. Slider-crankshaft engines in both cases the spark plug and compression ignition have been used for decades and they have many operation problems that require the thinking about better devices. Linear (free-piston) engines have a simple design (no crankshaft) that makes them able to be the engines of the future; they indicate high mechanical efficiencies due to the less friction losses, which are involved within their operation. In addition, these types of engines can be presented in many different applications like compressors, pumps and electrical generators. The ignition process in free-piston engines can be driven by either spark, compression or $\mathrm{HCCl}$ ignition methods. When the free-piston device is used with electrical generators, the $\mathrm{HCCl}$ combustion appears to be among the most suitable approaches [1].

In general, linear engine devices can be presented in two main configurations. The two cylinders' linear engine has two opposite combustion chambers that have ignition at each end; as a result, the compression ratio varies under the influence of combustion process primarily. The springs in the case of the two cylinders' linear engine have less stiffness since the strokes are completed due the pressure difference between the two cylinders. On the other hand, the single cylinder version needs far stronger springs because that is all that returns the piston along the compression stroke. As a result, the motion is far more determined by the springs than the combustion

The operation in free-piston engine can include cyclic variations since the device has no fixed top dead center (TDC) location and therefore a variable compression ratio. Working with variable compression ratio gives the flexibility to the interior system especially the $\mathrm{HCCl}$ process. This unique cycle to cycle freedom can be employed to serve the variable load requirements [1]. However, the cyclic variations increase the controlling demands since steady state performance is usually desired [2].

Chemical kinetics is not introduced widely in the free piston engines where the majority of the studies still use the empirical approach as tool to investigate the overall performance of the free-piston engine. However, using chemical kinetics models as the driving paddle of the piston dynamics is a new interesting task within the free piston models industry. In this research, the possibility of coupling the two polar models of the free-piston engine has been introduced. The study investigates the chemical kinetics approach to represent the $\mathrm{HCCl}$ combustion of the used fuels. However, the dynamic model is reflecting the outputs of this combustion model in addition to any future improvements. Following this introduction, the dissertation considers a comprehensive literature review for the different topics that belong to this research.

\subsection{Homogeneous charge compression ignition engines}

The spark ignition (SI) and the compression ignition ( $\mathrm{Cl}$ ) engines are still used and many modifications have been done in order to enhance their performance, reducing their emissions. $\mathrm{HCCl}$ combustion engines get a lot of attention because they are able to combine features from both of the spark and compression ignition engines [3].

The advantages of $\mathrm{HCCl}$ technology include the ability of operating at diesel-like stoichiometric compression ratios (>15), therefore; $\mathrm{HCCl}$ engines offer higher efficiencies (>40\%) than conventional $\mathrm{SI}$ engines [4]. Reducing the combustion peak temperature is one of the most important advantages of $\mathrm{HCCl}$ 
combustion, since harmful emissions like $\mathrm{NO}_{x}$ are reduced substantially. Not only the peak temperature reduction but also limiting the formation of the rich fuel areas is another important advantage of $\mathrm{HCCl}$. The nature of lean combustion, which introduced in $\mathrm{HCCl}$ engines, has a strong effect in decreasing the particulate matter significantly [3].

Combustion in $\mathrm{HCCl}$ engines needs the premixing of the fuel with the air. This premixing process is required to ensure the homogeneous combustion. Fuel-air mixing could happen either in the manifold or by the early direct fuel injection. Thereafter, the fuel-air mixture undergoes a compression process that will increase its temperature up to the auto-ignition point where the combustion will start. The nature of combustion in $\mathrm{HCCl}$ engines is considered as a rapid, sudden and simultaneous combustion burning process [3].

The ignition in the $\mathrm{HCCl}$ combustion case is completely controlled by the chemical kinetics of the fuel where it is controlled for example by the spark time in the case of the spark-plug engines. The oxidation of the fuel by the air is driven by the chemical reactions only. Such type of combustion can happen under two temperature regimes. The first is below $850 \mathrm{~K}$ (cool flame combustion), while the high temperature combustion is occurred around or above $1050 \mathrm{~K}$, between these two regimes the auto-ignition point can be defined. The reactions that are primarily responsible for the auto-ignition at high temperature are presented in equations (1.1) and (1.2) [5]:

$\mathrm{HO}_{2} \cdot+\mathrm{RH} \rightarrow \mathrm{H}_{2} \mathrm{O}_{2}+\mathrm{R} \cdot$

$\mathrm{H}_{2} \mathrm{O}_{2}+\mathrm{M} \rightarrow \mathrm{OH} \cdot+\mathrm{OH} \cdot+\mathrm{M}$

Where $\mathrm{R}$ is any hydrocarbon radical and $\mathrm{M}$ is any other molecules in the system. At the low temperature combustion, the decomposition of $\mathrm{H}_{2} \mathrm{O}_{2}$ is very slow; this leads to different chain branching reactions.

Mainly, fuel structure and engine conditions are responsible about the nature of the combustion. For example, long straight-chain alkanes and low research octane number (RON) fuels will undergo a cool flame combustion. However, branched-chain alkanes, aromatics and high RON fuels will follow a high temperature combustion process [6].

$\mathrm{HCCl}$ engines include many limitations ether when they are using highly lean or highly rich mixtures [3]. For example, very lean mixtures can lead to incomplete combustion events, which will result with low efficiency and relatively high levels of UHC and CO emissions. However, rich mixtures will probably end up with misfire problems. This can happen if the fuel-air composition does not reach the self-ignition conditions [3]. Since $\mathrm{HCCl}$ represents the knock phenomenon within the IC engine, it is possible to have a damage within the entire engine parts, especially if the ignition process is not controlled properly [7].

The previous difficulties require a proper controlling in those devices that use $\mathrm{HCCl}$ combustion. The inadequate control will not affect the system performance only, but it can lead to engine failure [2]. The nature of combustion in $\mathrm{HCCl}$ engines looks like a random, sudden and multiple auto-ignition events that occur throughout the combustion chamber. The locations where the hot gases and the fresh charge are interacted have the best chance to ignite first, while a fully uniform auto-ignition combustion can be observed also $[8,9]$.

Many studies like propagation speed investigation and spatial auto-ignition process improvements have significant rule in controlling $\mathrm{HCCl}$ combustion. Attempts to control the $\mathrm{HCCl}$ combustion using high compression ratios were applied. Planer laser induced fluorescence (PLIF) showed that the transition from fuel-air mixture to combustion products was following a gradual trend [10]. In addition, many 
mathematical analyses were performed to investigate the spreading of the high temperature spots in the combustion chamber. Those spots are caused by the fast chemical reactions toward the colder regions inside the engine cylinder $[11,12]$.

Using EGR is another technique that can affect the control of $\mathrm{HCCl}$ engines. The inhomogeneities that are caused due to the improper mixing between the EGR gases and the fresh charge found to have a significant effect on the auto-ignition process [10]. For example, when EGR gases are concentrated near the cylinder walls, while the fuel-air mixture is in at the center of the cylinder, a noticeable performance improvement was observed. In the opposite way, when the EGR gases are located near the center while the fresh charge is near the wall, a slower $\mathrm{HCCl}$ combustion will occur [3]. These discrepancies may return to many factors like, the differences in $\mathrm{CR}$, the inlet charge conditions or the mixing method between the gases $[3,4]$.

In. general, the operating range in $\mathrm{HCCl}$ devices is restricted by at low load by the misfire events which can happen, in the other side, the operation is limited by a high pressure rates when high loads are applied. Expanding the operating range of the $\mathrm{HCCl}$ engines is of one of the main concerns within these engines industry. For example, to extend the high-load operating limit, turbocharging and supercharging $\mathrm{HCCl}$ combustion techniques are considered usually [13].

\subsection{Review of literature}

\subsubsection{Linear engine:}

The concept of free piston engine is referred to as early as 1922, when Pescara [14] created his own free piston compressor driven by a compression ignition combustion. Later on, Pescara invention led to patent a multi-stage free piston air compressor in 1941 [15]. The free piston engine became a desirable device since it has a simple design and less mechanical losses. The 1990s of the last century had many developments in the free-piston engines applications like the electrical generators. Those machines got a lot of attention since free-piston engines were among the most suitable devices to run them. In 1995, a project funded by US army and granted to Southwest Research Institute and University of Texas at Austin explored the feasibility of using the engine within the hybrid electric vehicles [16]. By 1998, both of West Virginia University (WVU) and Sandia National Laboratories (SNL) had their own research projects in the free-piston engine field. SNL investigations were examining the idea of implementing $\mathrm{HCCl}$ combustion in free piston engines by using rapid compression machines. The modeling process was following a nearly constant volume combustion that achieved Otto cycle behavior [17]. Later on, they expanded their work by using numerical modeling of the system to investigate the feasibility of using $\mathrm{HCCl}$ combustion in their models [18].

At the same time, another study was going on in SNL to predict the effect of the scavenging process on both of the thermal and alternator efficiencies [19]. In this task, the result indicated that the cyclic variations have a little effect on the scavenging process as low temperature charge is used [20]. Nowadays, the researchers at SNL are examining newer engine versions; the engines come in the forms of twin and opposed piston arrangements. In the new system, the researcher included a single centrally located combustion chamber with outlying pneumatic bounce chambers to aid the controlling process [21]. SNL system is described in Figure 1 where the system components are illustrated. 


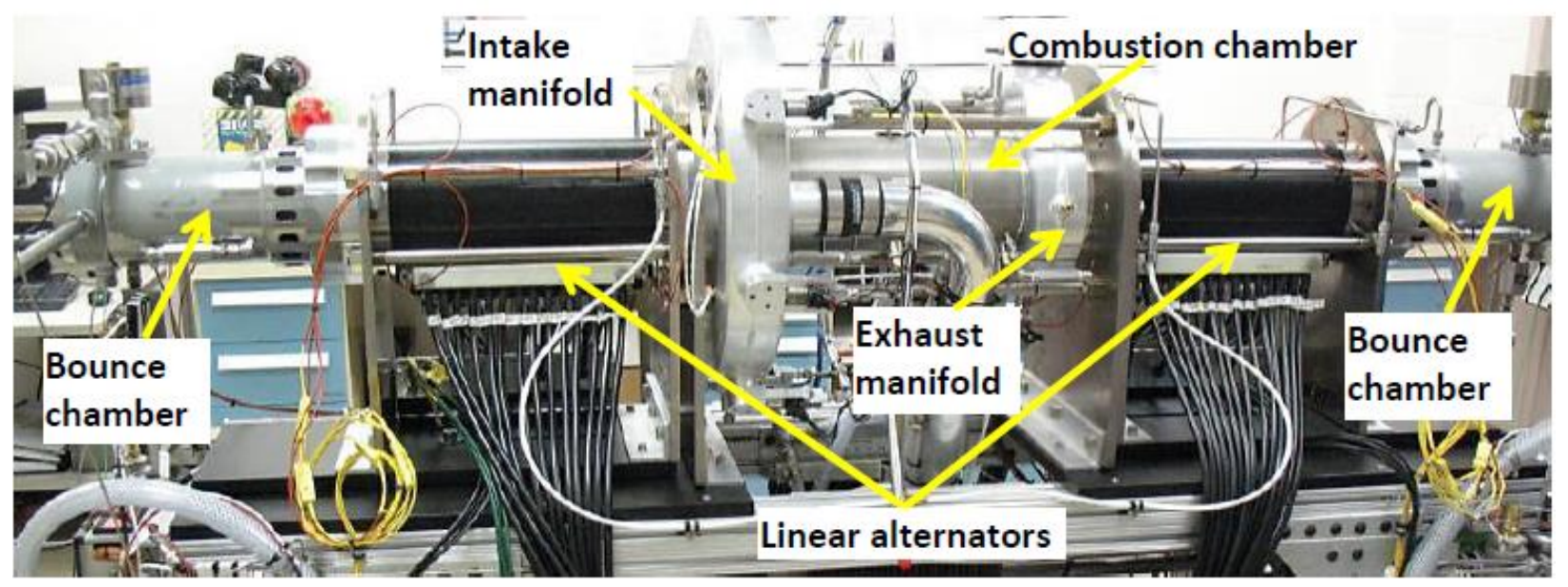

Figure 1 Sandia's Free Piston Engine - Linear Alternator Prototype [21]

WVU researchers started their approach by building a prototype for experimentations. WVU prototype was a gasoline fueled with $300 \mathrm{~W}$ power output. The stability of the engine was strongly depending in the combustion time. This prototype expanded the space of the parametric study by understanding the effects of many parameters. Peak pressure, engine frequency and compression ratio were examined under the influence of combustion duration. However, the translator mass effect was examined and it indicated a proportional relationship to the stroke length and the compression ratio at the engine load and intake conditions. In the hand, it has an inverse relation with the engine frequency $[22,23]$. System frequency and efficiency were evaluated based on the effects of the fuel-air ratio, injection time, frictional losses and geometry. At the same time, numerical investigations were performed for simulations verification [22]. These investigations were the core of the second prototype which was built with different specifications than the first prototype (Figure 2) [24]. The prototype represented a diesel fueled, boosted, direct injected, port scavenged and dual cylinder engine. It was operating within a frequency range of 50 to $60 \mathrm{H}_{z}$ [25]. 


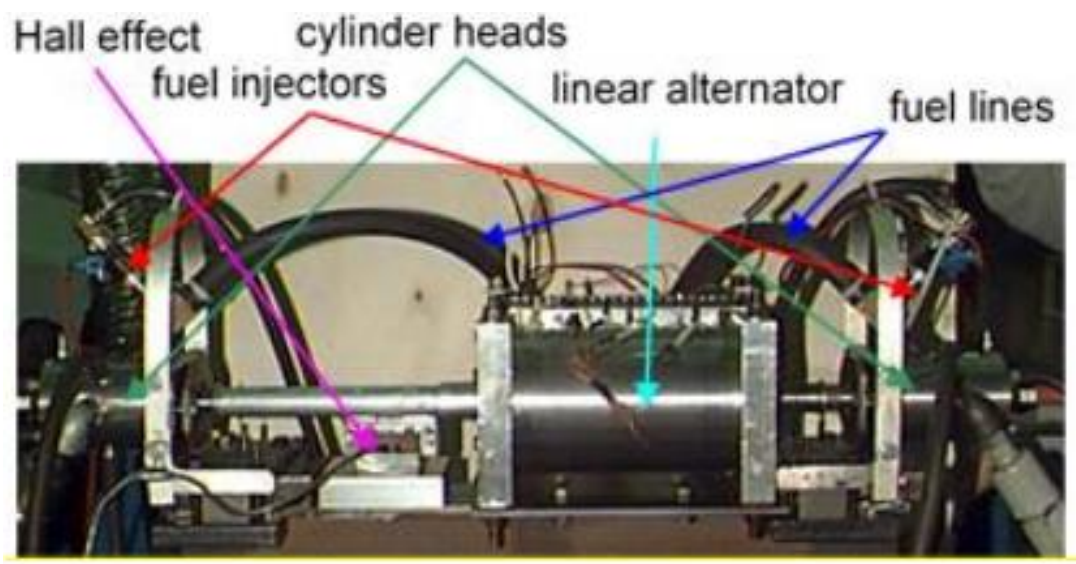

Figure 2 Early experimental LE setup in WVU [22].

Another prototype was built in 2007 by Newcastle University. The prototype has a single combustion chamber in addition to a bounce chamber for rebounding and therefore system control supporting. They were examining the feasibility of $\mathrm{HCCl}$ control [26]. Although the results indicated a difficulty in controlling the model, but wide opportunities were opened for testing $\mathrm{HCCl}$ combustion experimentally. At the same time, a series of implementations was applied to the model based on those results [27]. Variable operating conditions like SI [28] and $\mathrm{Cl}$ [29] were tested and explored within the study. However, introducing a bounce chamber with pressure profile and adding a dynamic translator were useful in controlling the operation [30, 31]. Figure 3 shows a schematic of Newcastle University prototype as found in [27]. Recently many articles were published by Newcastle researchers to review challenges like piston motion, heat transfer, vibrations, scavenging, lubrication and commercial expectations of the free piston engine [32].

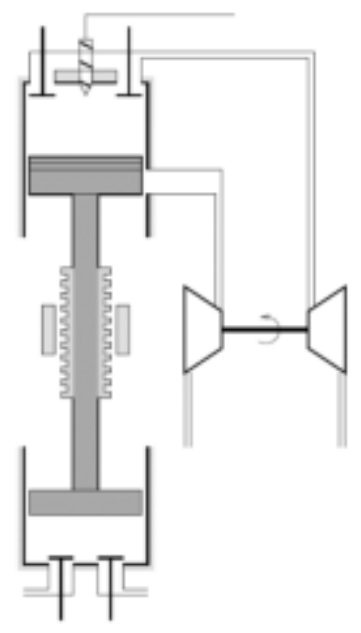

Figure 3 Device schematic of Newcastle University's free piston engine [27].

Free-piston engines researches have been done on many locations around the world, for example; Chalmers University of Technology simulated a dual cylinder linear engine to investigate multi-fuel capabilities of this device [33]. In addition, Pempek System (Australia) developed the free-piston 3 (FP3) 
concept which represents a package of three piston engines [34]. In France, a research done by IFP showed an efficiency within the range of $50 \%$ using high compression ratios and EGR [35]. The German Aerospace Center (DLR) developed an $8 \mathrm{~kW}$ prototype that includes a mechanical springs with an operating frequency of $20 \mathrm{H}_{\mathrm{z}}$ [36]. Toyota announced about a $10 \mathrm{KW}$ single cylinder linear engine, this engine will be used in hybrid vehicles as a system assistance device [37, 38]. By this time, a Korean group investigated the free piston engine using a dual cylinder, spark ignited propane fueled prototype. They explored the feasibilities of the system control and the possibility of transiting from spark to $\mathrm{HCCl}$ ignition approach [39].

Model control is one of the most important and difficult tasks in free-piston investigations. It was found that, some of the dynamical improvements have the potential to enhance the overall system performance. For example, using springs has dual benefits by tailoring the piston dynamic to match the $\mathrm{HCCl}$ combustion process and by supporting the piston during the compression stroke $[40,41]$

The thermodynamics model is the major concern in this research since it includes the chemical kinetics approach. However, the piston dynamics are also important as they present the supplementary part that will complete the operation cycle.

\subsubsection{Thermodynamics and chemical models:}

Based on the aims of the study, the available resources and computational time approaches can be considered from the least to the most complex [2]. Zero-Dimensional thermo-kinetics, quasi-dimensional thermo-kinetics, segregated, sequential fluid mechanics -thermo- kinetics multi-zone approach and multidimensional fluid mechanics with coupled kinetics are chemical kinetics models. They vary from the simple to the complex respectively [2,3]. Every model has its own advantages and disadvantages, in this section a brief literature review will be introduced for each model as long as some of their advantages and limitations.

\subsubsection{Zero- dimensional thermo-kinetic model:}

This model is considered as the simplest among the chemical kinetics models. In this model, a single zone approach is used to simulate the in-cylinder gas mixture. First law of thermodynamic is applied to the homogenous mixture of gases and for simplicity, the ideal gas law is considered. However, the effects of fluid mechanics are not considered specifically. For example, it may be included to simulate the heat transfer model when this model is required within the modeling process [2]. In addition, zero-dimensional models have the ability to capture the main system outcomes like, work output, combustion time and peak combustion pressure and temperature. On the other hand, these models are weak in detecting walls temperature effects. This fact makes them inadequate for emissions prediction since a single homogenous mixture is applied instead of multi-zones one $[2,3]$. In addition, zero-dimensional models are missing the ability of capturing the mixing process during the charge induction [2].

Goldsborough and Van Blarigan [18] at SNL simulated a free piston engine with high compression and low equivalence ratios. They used a zero-dimensional model with full kinetics details for the used hydrogen fuel. A full performance analysis was done to check the improvements and the limitations of the entire system.

Aceves, et al. [42] simulated a single cylinder engine using hydrodynamic, chemistry and transport (HCT) chemical kinetics code. The engine was working with 18:1 as a compression ratio at $300 \mathrm{~K}$ as an inlet 
temperature. The results indicated that the engine efficiency was within the range of $50 \%$ even when a variable compression ratio was applied, while the $\mathrm{NO}_{\mathrm{x}}$ emission was below $100 \mathrm{ppm}$.

Wong and Karim [43] studied the EGR effect on the performance of an $n$-heptane fueled engine. The study approach used an analytical single zone model with chemical mechanism of 683 reactions and 158 species. In the other side, Yamasaki and lida [44] examined an n-heptane fueled engine using CHEMKIN ${ }^{\circledR}$. Many parameters like, equivalence ratio, compression ratio, intake temperature and fuel composition were tested.

Fiveland and Assanis [45] built a single zone model for a four strokes engine fueled by hydrogen. The model describes the in-cylinder thermodynamics using a full detailed chemical kinetics approach. Physical models of the $\mathrm{HCCl}$ engine processes were built also to investigate the system. In their research, CHEMKIN $^{\circledR}$ was the framework of the fuel species reactions. Two mechanisms were implemented, a reduced mechanism with 11 species and 23 reactions and a full mechanism represented by the wellKnown GRI 3.0 mechanism.

\subsubsection{Quasi-dimensional thermo-kinetic model:}

The quasi-dimensional model represents potentially an extended zero-dimensional model that includes extra considerations by applying multiple zones in the engine cylinder $[2,3]$. One of the most important advantages of those models is that the effects of the temperature stratification and near the walls/crevice quenching can be derived and evaluated. This gives many improvements to the emissions prediction process compared to the zero-dimensional. However, this feature of the quasi-dimensional models adds extra computational cost and extra complexity to the applied approach [2].

Fiveland and Assanis [45] after their single zone model developed a quasi-dimensional model to study the performance and the emissions levels of a supercharged engine. The model that was built to fill the gap in the previous zero-dimensional one. It was able to predict emissions within 10-20\% for UHC and up to $50 \%$ for $\mathrm{CO}$ at turbocharged engine conditions. At Ford Motor company, Easley, et al. [46] tested two $\mathrm{HCCl}$ models, a single zone model and a quasi-dimensional one. Their simulations included a zerodimensional model to predict the main performance and to perform the parametric study. On other hand, a quasi-dimensional model employed for emissions prediction and heat losses calculations. The quasidimensional model in their work had four different zones, crevice, boundary layer, inner core and otter core. The heat transfer between the gas mixture and the environment through the different zones was considered in the all zones except the inner one. A study of the effect of the boundary layer in engine performance was done as well. It showed that CO emissions primarily arise because of the fuel flowing out of crevices and boundary layer. This fuel will be partially oxidized and therefore produces those emissions.

Node and Foster [47] examined the intake temperature effect on the $\mathrm{HCCl}$ combustion process. They compared that with the effect of the equivalence ratio at the same time. They used a multi-zone model for a hydrogen-fueled engine. Developing a full detailed chemical kinetics code linked with CHEMKIN ${ }^{\circledR}$ libraries was the basic platform of their model. The results showed that the inlet temperature has the dominant effect in controlling the $\mathrm{HCCl}$ combustion process. They believed that introducing appropriate temperature inhomogeneity to the in-cylinder mixture would help achieving a stable and steady performance. 


\subsubsection{Segregated, sequential fluid mechanical - thermo-kinetic multi-zone approaches}

Mixing process between gases prior combustion is the main aim of these approaches. Chemical kinetics are tightly coupled with those models. Fluid mechanics solver is used also to model the reactants and the diluted gases distributions based on sequestering the gases into number of zones [2]. After that, the combustion process is simulated using a multi-zone approach as the one used in quasi-dimensional models. The main advantage of this type of models is the ability to model the mixing process during the induction stage, so that the effects of the inhomogeneity can be explicitly captured $[2,3]$.

Babajimopoulos, et al. [48] applied this approach to study the effect of the valve events and the gas exchange process in the $\mathrm{HCCl}$ engine simulations. A multi-dimensional fluid mechanics code (KIVA-3V) was used to model the whole process. Starting from the intake process passing by the compression one up to the transition point before the combustion, all of that was covered by the introduced multi-zone model. The model indicated that late intake valve opening has a potential effect in $\mathrm{HCCl}$ combustion control.

\subsubsection{Multidimensional fluid mechanics with coupled kinetics}

This approach is the most complex and computationally intensive among the thermo-chemical models. A full coupling process between the chemical kinetics and the fluid mechanics is required. Parallel processing is required in order to simulate the whole processes of the combustion and the fluid motions in the three dimensions. This fully coupled model has the ability to predict accurately the rate of representation of the composition, temperature inhomogeneities and $\mathrm{NO}_{\mathrm{x}}$ and soot emissions [2].

Kusaka, et al. [49] performed a numerical study on the combustion and the exhaust gas emissions for a duel natural gas engine using a multi-dimensional approach. A modified KIVA-3 ${ }^{\circledR}$ and $\mathrm{CHEMKIN}^{\circledR}$ subroutines present the model. It describes the oxidation process of the high carbon number fuels. The results indicated that EGR combined with preheating could favorably reduce $\mathrm{NO}_{x}$ and UHC emissions under the fact that the combustion process is moved from the flame propagation to the $\mathrm{HCCl}$ one.

\subsubsection{Thermo-chemical models used in free-piston engines}

Models that describe the heat release in the free piston engines could depend on ether experimentalempirical correlations like Wiebe functions or on detailed chemical kinetics [3]. In the first approach, many assumptions are applied to predict the combustion process. Usually in those models, enhancing the incylinder modeling process is not a main target of the study [2]. However, assuming combustion profiles will simplify the problem simulation and will increase the flexibility of the whole system to include more parameters effects. It will also give more space to validate the results with similar models in the literature [1].

Based on a proper reviewing of the literature, it was found that chemical kinetics models are not used effectively in the free piston engine simulations. The full coupling between the dynamic and the thermal models of those engines is a new task. In addition, $\mathrm{HCCl}$ combustion occurs in a very quick and sudden manner and it is fully defined by the fuel chemistry rather than the $\mathrm{SI}$ or $\mathrm{Cl}$ timing [50].

\subsubsection{FPE with $\mathrm{HCCl}$ combustion modeled by HCT software for chemical kinetics}

In 1999, Goldsborough and Van Blarigan [18] at SNL used a zero-dimensional model (mentioned before) to represent the in-cylinder thermodynamic processes in a free piston engine. The model was one of the first serious attempts to implement detailed chemical kinetics in the free piston engine simulations. Using an initialization scheme to start the simulation process was the basic step in their work. The bulk of 
the computational time was used to run this scheme. In the initialization scheme, a constant volume combustion process is assumed. An auto-ignition point is guessed near the TDC. After that, they implemented this initialization code to the HCT software which solves for the species reaction of the used fuel. The engine cycle was completely driven by $\mathrm{HCCl}$ combustion of $\mathrm{H}_{2}$ as a fuel. A tuning process was used to calibrate the engine performance toward a certain equivalence ratio. In addition, TDC combustion and desired compression ratio were calibrated. A scavenging model was used to simulate the exhaust gases blowdown process. The model was tested under different scavenging efficiency values. The results were basically focusing on the conceptual aspects of the engine. A full comparison between the free piston engine and the conventional crankshaft one was performed to show the differences between both of the engines operation. At the same time, a basic parametric study was done by testing a range of different values of the equivalence ratio, the scavenging efficiency and the CR. The dependency between the previous parameters and their effects on the overall system performance were also investigated. Goldsborough and Van Blarigan study could be concluded in five major points as following:

- $\quad$ The $\mathrm{CR}$ of the engine is varying and it depends mainly on the input parameters. Fuel equivalence ratio, intake temperature and scavenging efficiency are among the effective parameters. Good controlling of the compression ratio can lead to higher thermal efficiency if the input parameters are adjusted correctly.

- The $\mathrm{HCCl}$ combustion process is a very rapid process; this fact gives the flexibility of using the constant volume combustion assumption at the initialization scheme. This feature can help reaching higher thermal efficiencies, at the same time; the combustion duration will have less effect on the system performance.

- Working under relatively low equivalence ratios has a strong effect on the $\mathrm{NO}_{\mathrm{x}}$ emissions levels, this makes the $\mathrm{HCCl}$ approach suitable in such cases, especially that free piston engines have less friction losses than the conventional crankshaft engines. On the other hand, oxidation catalysts for UHC and $\mathrm{CO}$ emissions can be used since no exhaust gas after-treatment is required.

- $\quad$ Scavenging has a significant effect on the engine efficiency. The introduced losses by this process should be controlled by enhancing the overall engine dynamic design.

- The $\mathrm{HCCl}$ combustion process is sensitive to the piston dynamics where early combustion (before TDC) situation can lead to poor combustion efficiency. However, late combustion (after TDC) process has the ability to increase the $\mathrm{NO}_{x}$ emissions.

\subsubsection{FPE simulation using KIVA $^{\circledR}{ }^{-}$SENKIN ${ }^{\circledR}$}

$\mathrm{Li}$, et al. [51] published an article in 2008, which is describing the modeling of two-stroke free piston engine coupled with electrical generator. The thermodynamic model was built based on KIVA ${ }^{\circledR}$ and SENKIN ${ }^{\circledast}$ codes. An iteration scheme was used to initialize the engine cycle operation. Figure 4 shows the flowchart of their modeling process. 


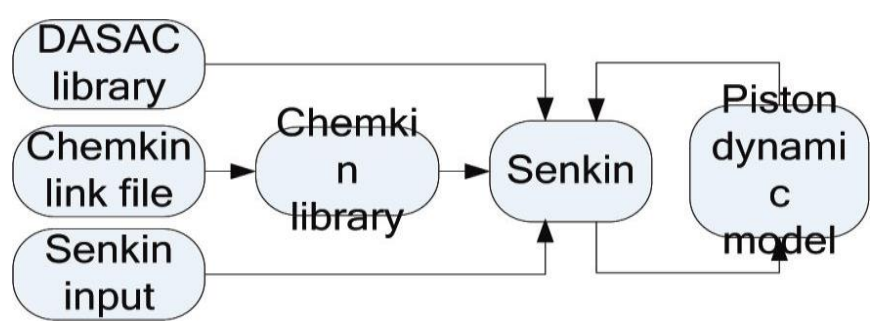

Figure 4 Associated input and output files for the chemical kinetic model [51]

In the initialization scheme, the piston dynamics were driven using empirical model to simulate the combustion process. This model was developed using Wiebe functions as a substitution of the chemical kinetics at this stage. Then, an iteration process starts between the piston dynamics and the chemical kinetics model until a steady state level is reached. A comparison between the crankshaft engine and the free piston one was done and the results of the study were as following:

- The piston acceleration profile showed that the free piston engine has almost three times the acceleration of the conventional engine, this decreases the residence time at the TDC, which leads to lower peak temperature.

- TDC Investigations indicated that under variable external loads, the TDC location could be changed. Based on this and with using lean mixtures, higher compression ratios can be obtained that leads to higher thermal efficiencies.

- The engine model has a shorter burning time at high compression ratios than this at low compression ratios. The peak pressure and temperature are less in the case of the free-piston engine than those of the traditional engine. In addition, a reduction in the temperature-dependent emissions level is also obtained.

\subsubsection{FPE modeling using CANTERA $^{\circledR}-$ MixMaster $^{\circledR}$}

Recently in 2015, Zhang et al [52] published a new model for linear engines simulation under $\mathrm{HCCl}$ conditions. The model was built with a very similar way to the previous two models by using Cantera platform with MixMaster ${ }^{\circledR}$ to represent the chemical kinetics. The flowchart of their model is shown in Figure 5 that describes the model-working path. Simple and variant piston profiles are employed within the gas dynamic model. An iteration between the gas dynamic model and the kinetics model is applied until the steady state point, at the same time, a pre-described piston profile is assumed to initialize the simulation. The piston motion profile is given by equation (1.3), the piston motion is assumed to follow an elliptical profile based on this equation. In the mentioned equation, $\mathrm{A}$ and $\mathrm{B}$ refer to the BDC and TDC locations respectively, while $f$ is the engine frequency, $\Omega$ is the ellipse ratio (minor axis/major axis), it describes the shape of the ellipse and $t$ is the time. 


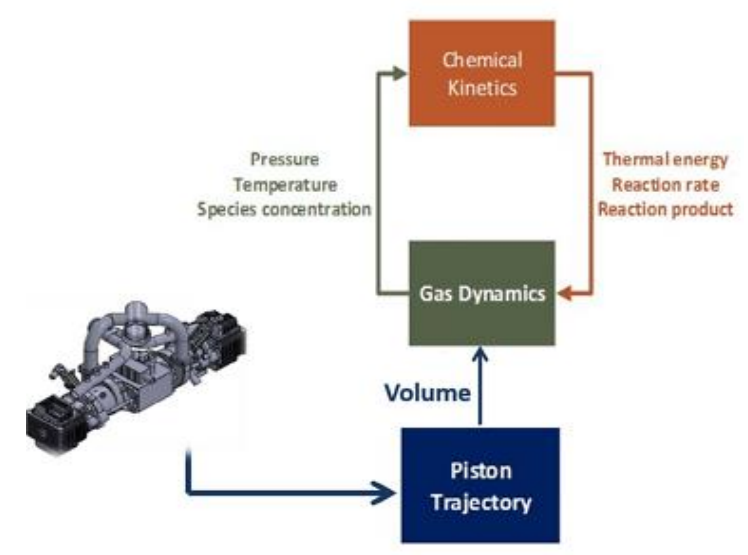

Figure 5 Interaction between chemical kinetics and gas dynamics [52].

$$
X=\frac{A \cdot \Omega \cdot \cos (2 \pi f \cdot t)}{\sqrt{\Omega^{2} \cdot \cos (2 \pi f \cdot t)^{2}+\sin (2 \pi f \cdot t)^{2}}}+B
$$

Six different types of fuels in addition to many piston motion profiles were tested and compared. The results indicated that variable compression ratios could be achieved. Therefore, high thermal efficiencies and less emissions levels could be obtained if a proper control is applied.

\subsubsection{Heat transfer in IC engines}

In engines design and modeling, heat transfer is playing a significant role; it is directly affecting the thermal and mechanical efficiencies. Heat transfer in internal combustion engines is considered always as heat loss from the engine cylinder walls, piston ...etc. to the outside environment. Many models have been modified to describe the heat loss in IC engines. Empirical formulas have been derived and investigated within numerous models that vary in complexity and purpose of use [6]. Generally, heat transfer correlations in internal combustion engines are divided upon three main groups. Time- averaged, instantaneous spatially averaged and instantaneous local heat flux approaches $[6,7]$.

\subsubsection{Time- averaged correlations}

In this type of correlations, the temperatures of the coolant flow under variable operation conditions is calculated. Based on that, an averaged time heat flow can be derived leading to averaged heat transfer coefficient [6]. Basically, the heat transfer coefficient is expressed as groups of non-dimensional numbers which describe the nature of heat transfer process in the system [6].

Taylor and Toong [53] in 1957 derived a formula which coupled the Nusselt's number ( $\mathrm{Nu}$ ) and Reynolds's number ( $R e$ ) based on different engines readings. Spark ignition with air and water coolants engines were used in their study. $\mathrm{Cl}$ engines with water as a coolant liquid were evaluated as well.

Heywood [6] and Annand [54] obtained data from operating engines over a wide range of piston speeds and intake conditions like the fuel-air ratio. An expression with the following type had been proposed:

$\mathrm{Nu}=\mathrm{aRe}^{\mathrm{n}}$

$\mathrm{Nu}=\frac{\dot{Q} \mathrm{~B}}{\mathrm{~kg}_{\mathrm{g}} \mathrm{A}_{\mathrm{P}}\left(\mathrm{T}_{\mathrm{g}}-\mathrm{T}_{\mathrm{c}}\right)}$ 
$\operatorname{Re}=\frac{\dot{m}_{\mathrm{g}} \mathrm{B}}{\mu_{\mathrm{g}} \mathrm{A}_{\mathrm{P}}}$

Where $Q$ is the heat transfer rate to the coolant, $m_{g}$ is charge mass flow rate, $A_{p}$ is piston projected area, $B$ is the cylinder bore, $\mathrm{k}_{\mathrm{g}}$ is the thermal conductivity, $T_{\mathrm{g}}$ is the mean effective temperature of the engine and $T_{c}$ is the coolant temperature. The factor $a$ in equation (1.4) represents the effect of Prandtl number $(\mathrm{Pr})$, while both of the factors $a$ and $m$ are chosen based on the best fit of an experimental data.

Following the expression in equation (1.4), Taylor and Toong [53] found that the Nu number is related to Re number as:

$\mathrm{Nu}=10.4 \mathrm{Re}^{0.75}$

\subsubsection{Instantaneous spatially-averaged coefficient correlations}

In this approach, a quasi- steady assumption is used to find the heat transfer coefficient. The heat transfer is proportional to the temperature difference between the cylinder walls and the coolant fluid. The instantaneous gas temperature is assumed to be uniformly distributed along the heat transfer area. Annand [55] commented in this approach because of the fact that there is no theoretical or experimental opinions that support it. Although the approach is accepted and is used widely, the comment came from the idea that heat transfer varies from cycle to cycle in the IC engines. In addition, the heat capacity of the working fluid causes a phase lag between the temperature difference and the heat flux at the wall-fluid interface.

Eichelberg [56] is considered among those who did early attempts to predict the heat loss in reciprocating engines. His equation for the heat transfer coefficient had been modified later on by Stone [7], Annand [54]and Woschni [57]. The equation is given with the following form:

$\frac{\dot{\mathrm{Q}}}{\mathrm{A}}=2.43 \mathrm{~V}_{\mathrm{P}}^{1 / 3}\left(\mathrm{pT}_{\mathrm{g}}\right)^{1 / 2}\left(\mathrm{~T}_{\mathrm{g}}-\mathrm{T}_{\mathrm{s}}\right)$

$\mathrm{h}=\frac{\dot{\mathrm{Q}}}{\mathrm{A}\left(\mathrm{T}_{\mathrm{g}}-\mathrm{T}_{\mathrm{s}}\right)}$

Where $A$ is the instantaneous surface area, $T_{g}$ is the instantaneous bulk temperature, $T_{s}$ is the mean surface temperature, $p$ is the mean cylinder pressure and $V_{p}$ is mean piston speed. Woschni [57] presented a modified equation later on to combine the radiations effects by notifying the importance of the $\mathrm{pT}_{\mathrm{g}}$ term in the old equation:

$\mathrm{h}_{\mathrm{c}}=1.14 \cdot 10^{-5} \cdot\left(1+1.24 \mathrm{~V}_{\mathrm{P}}\right)\left(\mathrm{p}^{2} \mathrm{~T}_{\mathrm{g}}\right)^{1 / 3}$

Another widely used equation is the one obtained by Woschni [57]. This equation and those which were mentioned before share the forced convection concept. The equation has a power law profile. At the same time, it was found that it has less effect in modeling the heat transfer at certain time ranges like after ignition and during the expansion stroke [2].

$N u=0.035 R e^{0.8}$

In this study, a modified version heat transfer correlation of Woschni equation has been used. It specifically modified for the free piston applications as mentioned in the reference where it has been used.

\subsubsection{Correlations for the instantaneous local heat transfer coefficient}

Correlations under this approach were modified to fit specific locations inside the combustion chamber like the piston or the cylinder walls. LeFeuvre, et al [58] modified an equation that describes the 
instantaneous walls heat flux at the cylinder head and the piston. The equation measures the heat loss rate at different radial locations along the bore axis.

$\dot{q}=a \frac{k_{g}}{r} \operatorname{Re}^{0.8} \operatorname{Pr}^{0.33}\left(T_{g}-T_{s}\right)$

Where $T_{s}$ refers to the walls temperature. Dent and Suliaman [59] in 1977 proposed a newer version of the previous equation by taking $\operatorname{Pr}$ as constant and equal to 0.73 which led to the following equation:

$\dot{q}=0.023 \frac{k_{g}}{r}\left(\frac{\omega_{g}^{2} r}{v}\right)^{0.8}\left(T_{g}-T_{s}\right)$

Where $\omega_{\mathrm{g}}$ and $\mathrm{r}$ are the relevant velocity and the desired radius respectively.

\subsection{5 $\mathrm{HCCl}$ control}

$\mathrm{HCCl}$ combustion control is a difficult and complicated task within the IC engines operation. Closed control loop is the most known method in controlling the $\mathrm{HCCl}$ process. Agrell, et al. [60] modified a controlling scheme by implementing variable valve timing that tunes the compression ratio and controls the time of ignition. Haraldsson, et al. [61] developed a control model by changing the fuel ratios in a mixture of two fuels that will make the work output vary as the combustion time is controlled. Olsson, et al. [62] used the same technique but with changing the engine CR instead of the fuel ratio. This change resulted with a shifting in the ignition point. A physics-based approach is another method in $\mathrm{HCCl}$ control models. Input-output controller can calibrate the $\mathrm{HCCl}$ process by affecting the process dynamics using the input parameters like those of the engine geometry and fuel system [2].

\subsubsection{Natural gas fuels}

Many studies show that natural gas can be represented by components of methane, ethane, and propane. Methane concentrations can be as low as $74.5 \%$ (by volume) while ethane and propane concentrations can reach as high as $13.3 \%$ and $23.7 \%$, respectively._Remaining percentages are typically made up of higher order hydrocarbons, carbon dioxide, oxygen, nitrogen, and hydrogen sulfide [63, 64, $65]$.

For natural gas fueled combustion engines, significant enhancement in the ignition of methane-based fuels can be achieved using higher hydrocarbons additives [64]. An important environment to investigate the significance of fuel additives is found in engine systems where variations in auto-ignition phenomena are significant to performance. Combustion phenomena such as flashback and flame stabilization can also be of major concern. In some cases, the flame propagation speed will be more than speed of the incoming reactants to the flame zone. In this case, the flame will be speared randomly which is known as a flashback [66].

In natural gas fueled combustion engines, the methane number of the fuel is a good indicator for knock resistance. Knock resistance is an important characteristic of fuel within the engine and it defines the upper compression limit of the engine that is strongly tied to the efficiency. A fuel with high knock resistant has a low propensity to auto-ignite and it enables the engine to operate at higher compression ratios. When calculating the methane number, the highest possible index is achieved by pure Methane; it is given the value of 100. In contrast, pure $\mathrm{H}_{2}$ fuel is given an index of zero, this means that Methane has the highest knock resistance and $\mathrm{H}_{2}$ has the lowest $[67,68]$.

Compared to the other industry standard fuels, the Methane number (MN) for natural gas can be represented using the motor octane number (MON). This is demonstrated in equations (1.14) and (1.15) 
[68]. In the calculations, $H / C$ presents the ratio between hydrogen and carbon atoms within the fuel molecules.

$M O N=-406.14+5080.4 \cdot(H / C)-173.55 \cdot(H / C)^{2}+20.17 \cdot(H / C)^{3}$

$M N=1.624 \cdot M O N-119.1$

In the current dissertation, the fuel combustion consists of a mixture of gases: Methane $\left(\mathrm{CH}_{4}\right)$ at $80 \%$, Ethane $\left(\mathrm{C}_{2} \mathrm{H}_{6}\right)$ at $11 \%$, and Propane $\left(\mathrm{C}_{3} \mathrm{H}_{8}\right)$ at $9 \%$, which refer to a basic natural gas composition based on US department of energy [69]. Such fuel composition represents a fuel with a low methane number [70]. The addition of ethane and propane fuel components acts to reduce the knock resistance of the fuel and assist in the ignition process. Low knocking resistance reduces the compression required within the free piston linear engine, and enables a wider set of input parameters for successful engine operation with $\mathrm{HCCl}$ combustion.

\subsubsection{Exhaust gas recirculation}

\subsubsection{EGR review}

EGR is not a new technology in reducing engines emissions; it has a noticeable effect in reducing $\mathrm{NO}_{\mathrm{x}}$ emissions specifically. This fact made it as one of the most interesting techniques in emissions reduction field [71].Many researches showed that, a lower flame temperature could be achieved when lower specific heat ratio is introduced. EGR includes $\mathrm{H}_{2} \mathrm{O}$ and $\mathrm{CO}_{2}$ that will reduce the specific heat ratio to levels less than those found at air only. The increasing in the specific heat capacity and therefore the reduction in the heat capacity ratio will end up with less flame temperatures. This fact is the key in $\mathrm{NO}_{x}$ reduction since those emissions is highly depending on the flame temperature. Using some $\mathrm{CO}_{2}$ within the intake charge by recycling a fraction of the exhaust gas can lead to a higher specific heat capacity. Concurrently, the EGR will dilute the oxygen $\mathrm{O}_{2}$ concentrations within the intake charge. Such observation is noticed in lean mixtures combustion where the exceeded air will cool the mixture bulk temperatures. The mentioned benefits of EGR have the potential to reduce the $\mathrm{NO}_{x}$ emissions outputs significantly $[72,73,74]$

$\mathrm{HCCl}$ combustion method produces ultra-low $\mathrm{NO}_{x}$ levels and near zero soot emissions. It provides equal or greater fuel conversion efficiencies compared to that of the conventional combustion methods $[75,76,77]$. Many reasons explain such features of $\mathrm{HCCl}$ combustion, at first, the nature of the multi-spot spontaneous combustion associated with the $\mathrm{HCCl}$ combustion. This refers to the lean and premixed fuelair that produces high heat release rate (HRR) with low flame propagation. Another reason is the higher thermal efficiency due to the higher compression ratio values that can be used. This factor represents the close to constant volume, ideal Otto cycle heat release that helps in converting the fuel within a very rapid process $[77,78]$. However, the lack of the proper control for the ignition time, in addition to the difficulty in achieving high engine loads cause a relatively high $\mathrm{UHC}$ and $\mathrm{CO}$ emissions in $\mathrm{HCCl}$ devices [78].

In conventional spark and compression ignition engines, EGR is used to decrease the combustion peak temperature which lead to lower $\mathrm{NO}_{x}$ emissions levels. The used amount of EGR has many limitations within the $\mathrm{SI}$ and $\mathrm{Cl}$ engines. among the main limitations are the misfiring and high formation levels of $\mathrm{UHC}$ and $\mathrm{CO}$ emissions, the slow oxidation rates and the lower flame temperatures are common reasons for that. Such limitations are expected to be wider in the $\mathrm{HCCl}$ combustion machines where EGR implementation have been investigated but not commercialized yet. Furthermore, excessive EGR affect engine durability and performance. The corrosive and abrasive components like sulfur oxide increase the piston-cylinder liner wearing [79]. 
Some investigations were done by Zhao et al. [80, 81], in their study, different EGR effects had been tested like the charge heating, dilution, heat capacity, chemical and stratification effects. Their study indicated that the charge heating has the tendency to advance the auto-ignition process, increase the gas HRR and decrease the burning duration. On the other hand, EGR dilution increases the combustion time and slow down the heat release rate, but it has no effect on the auto-ignition process. The heat capacity works in an opposite way to the chemical effect where the first one increases the burning duration while the other reduces it slightly. The stratification effects are concluded by presence of a high temperature mixing regions. Basically, those regions describe the location where the hot gases meet the fresh charges. On the other hand, excessive EGR will cause high dilution and low concentration of $\mathrm{O}_{2}$. As a result, unstable combustion, misfiring, and a high UHC and CO emissions levels were observed within the operation [81].

Machrafi et al. [82] studied the influence of the chemical aspect of EGR on the fuel auto-ignition. Their results showed that $\mathrm{OH}$ radicals define how the reactivity of the auto-ignition is changed. The dilution done by $\mathrm{N}_{2}$ and $\mathrm{CO}_{2}$ of external EGR increases the amount of the UHC emissions since $\mathrm{O}_{2}$ concentrations will be reduced. By the same way, the high EGR dilution decreases the peak temperature; this leads to have incomplete combustion that causes an increasing in CO concentrations [83].

\subsubsection{EGR on free-piston engines}

Limited $\mathrm{HCCl}$ studies that include the effect of EGR is found in the literature. One of those studies is the study done by both of Xutao and Wangyang [84]. In their study, a model of two strokes free-piston engine was used with $\mathrm{HCCl}$ combustion. They implemented a CFD code to simulate the combustion of dimethyl-ether (DME) using a full reaction mechanism. The implementation of EGR technique was to investigate its effect on the knock resistance. Based on their results, low knock resistance was achieved at higher EGR ratios. In the other side, low thermal efficiency was noticed when excessive EGR is used. The study does not consider $\mathrm{NO}_{\mathrm{x}}$ emissions as it assumed that $\mathrm{HCCl}$ combustion has low $\mathrm{NO}_{\mathrm{x}}$ emissions.

In current research, the EGR effect on the free-piston performance has been investigated by comparing a range of EGR ratios. EGR ratios up to $30 \%$ of the exhaust gas have been used and compared to no-EGR states. The resulted have represented in two paths, the first by assuming a constant intake temperature. In this case, the EGR effect has been predicted solely. In the other side, multi-intake temperatures have been assumed to demonstrate the effect of the coold and hot EGR charges. Through the study, the EGR has been implemented based on a constant mixture mass where the $\mathrm{O}_{2}$ molecules are replaced partially by $\mathrm{CO}_{2}$ and $\mathrm{H}_{2} \mathrm{O}$ molecules, while $\mathrm{N}_{2}$ concentration is constant before and after the EGR adding.

\subsubsection{Intake temperature effect on engine performance}

A few studies accounted the importance of the of intake air temperature in defining the characteristics of the $\mathrm{HCCl}$ combustion. Intake temperature investigations can help deeply in enhancing the overall performance of the free-piston engine especially in the controlling side. Zhang et al [85] investigated the effects of the intake air temperature and the equivalence ratio on the combustion process and emissions formation. In their model that represents a four-stroke $\mathrm{HCCl}$ diesel engine, the engine was fueled with many fuels like, ethanol, methanol and gasoline to perform the system performance at a wide fuel range. Their results indicated increasing the intake temperature advances the compression ratio and therefore the in-cylinder pressure. In addition, $\mathrm{CO}$ and $\mathrm{UHC}$ emissions formation was found to decrease at relatively high intake temperatures, while $\mathrm{NO}_{\mathrm{x}}$ emissions start to slightly 
increase, as gasoline is the fuel. Maurya et al [86] investigated the effects of intake air temperature and equivalence ratio on the $\mathrm{HCCl}$ combustion. Their model parameters were evaluated using a direct injection diesel engine with Ethanol as a fuel. Stable ignition time was achieved at range of $\lambda=2$ to 5 where the engine speed was $1500 \mathrm{rpm}$. Very low $\mathrm{NO}_{x}$ emissions levels were observed at all stable operating conditions; however, CO and UHC emissions were at higher values. Lida et al [87] in their study investigated the effects of the intake air temperature, compression ratio, equivalence ratio and the engine speed on the operational range of $\mathrm{HCCl}$ combustion engine. The engine was a single-cylinder, four-stroke cooperative fuel research (CFR) engine fueled with n-butane. In their study, the $\mathrm{HCCl}$ operation was achieved at 2000-rpm engine speed, $400 \mathrm{~K}$ intake air temperature and at 16.55 as a CR. Lower mean effective pressure (IMEP) and the specific fuel consumption were obtained. Those values for $\mathrm{HCCl}$ combustion were much less in comparison to the $\mathrm{SI}$ and $\mathrm{Cl}$ engine models. UHC and CO emissions were found to decrease when a greater load is applied. Recently in 2015, Cinar et al [88] performed a study to investigate the effects of the intake air temperature on the in-cylinder pressure, the HRR, the ignition time and the combustion duration. The model of the study was, a single-cylinder, four-stroke $\mathrm{HCCl}$ gasoline engine with two different excessive air coefficients. At $1200 \mathrm{rpm}$, a fuel blend of $20 \% \mathrm{n}$-heptane and $80 \%$ isooctane fuels was investigated under $\mathrm{HCCl}$ conditions. A range of Intake air temperatures from $40{ }^{\circ} \mathrm{C}$ to $120^{\circ} \mathrm{C}$ was applied to examine the effect of this parameter. Their results indicated that in-cylinder pressure and the HRR increased with the increasing in the intake air temperature. However, the combustion duration decreased in with gradual trend. In addition, they found that specific fuel consumption and the $\mathrm{NO}_{x}$ formation tend to increase at intake temperatures above $100{ }^{\circ} \mathrm{C}$. $\mathrm{CO}$ and $\mathrm{UHC}$ emissions were firstly increasing and then started to decrease at intake temperatures more than $90^{\circ} \mathrm{C}$.

In the case of the free-piston engine, it was found that there is no specific investigations that declare the effect of any of the intake temperature or the equivalence ratio on the system performance. In the coming sections, both of the intake temperature and the equivalence ratio effects on the free-piston engine will be discussed in details.

\subsubsection{The equivalence ratio effect on engine performance}

Many investigations have been done to predict the effect of the equivalence ratio on the $\mathrm{HCCl}$ combustion engines. Ghafouri et al [89] examined the effect of variable equivalence ratios on $\mathrm{HCCl}$ engine using chemistry based CFD code. They observed that increasing the equivalence ratio has the ability to delay the combustion process. They referred that to the compressive heating effect which is occurred at lower intake pressures. In addition, higher peak pressure was observed at higher equivalence ratios. They explained that by the high fuel energy that found at such high equivalence ratios. Another study by Rahbari [90] used $\mathrm{HCCl}$ engine fueled by ethanol. It was found that the burning duration decreased at high equivalence ratios due to the high reactions rate within the combustion process. The study indicated variations in the in-cylinder temperature as lean mixtures were implemented. A drop in the peak temperatures was noticed for those mixtures burning process. In the other side, less peak pressure was obtained when low equivalence ratios are applied especially for ultra-lean conditions. Another study done by Hamada et al [91] showed that higher peak temperatures would be observed when high equivalence ratio is used. That is attributed to the increasing in reactions rate of fuel oxidation. Therefore, a greater HRR will be obtained due to the higher presented fuel density. From emissions perceptions, the study indicated a proportional increasing in the $\mathrm{CO}$ and $\mathrm{UHC}$ emissions intensity to the equivalence ratio. The authors returned that to the higher combustion temperatures found at such conditions. 


\subsubsection{Ultra-lean combustion}

Ultra-lean combustion (i.e., $\phi \leq 0.5$ ) is considered as one of most effective techniques in designing low emissions engines. The engines that can operate under ultra-lean conditions will have the lowest combustion peak temperatures [92]. Practically, this is the effective mean of minimizing $\mathrm{NO}_{x}$ formation within the engine. The relation between the $\mathrm{NO}_{x}$ formation and the combustion temperature refers to the fact that when the combustion temperature is low enough, thermal $\mathrm{NO}_{\mathrm{x}}$ formation rate is too slow and therefore the engine emissions will be almost near zero [93]. In addition, ultra-lean burn will improve the engine thermal efficiency by improving combustion quality, reducing the heat transfer loss and extending the range of the CR [93]. However, ultra-lean operation includes higher cyclic variations and more instability in the combustion than the stoichiometric operation. Therefore, ultra-lean operation will be efficient with light loads systems since at high loads, many difficulties are presented like those that belong to the system stability and control [94].

Ultra-lean combustion is investigated limitedly within free-piston devices work since this kind of combustion can increase the control challenges that are already presented in free-piston engine. Experimentally, Greenwood et al [95] demonstrated the performance of a Hydrogen-Ethanol fueled engine using ultra-lean charges. They found that that Hydrogen addition increases the lean operating range. This lean operation helped in reducing $\mathrm{NO}_{x}$, stabilizing, and accelerating the combustion process. In addition, the reduction in $\mathrm{NO}_{x}$ was comparable to the efficiency of modern three-way catalyst and could be a good alternative to the current $\mathrm{NO}_{x}$ reduction technologies. Power output, thermal efficiency, and volumetric efficiency were not affected by the ultra-lean conditions when fixed equivalence ratio was applied. However, a reduced power output and low thermal efficiency were observed at over-limit hydrogen addition. Another study done by Aliramezani et al [94] showed that using ultra lean HydrogenMethane blends results with higher thermal efficiency that is limited in stoichiometric mixtures where more knock tendency is found.

\subsubsection{Fuels and fuels mechanisms}

\subsubsection{Fuels}

In this section, some of the fuels that have been used in modeling the current free-piston model are introduced. The chemistry between the fuel species play a significant role in defining the ignition in $\mathrm{HCCl}$ combustion devices. Examining the engine performance under the influence of different types of fuels can help the engine design to avoid many problems like high emissions and the low efficiencies [96]. Natural gas for example is consisting of Methane mainly; this fuel is used widely within internal combustion devices. The flexibility in storage and transport in addition to the less emissions compared to coal are among the advantages of this gas. In the other side, the products of the natural gas burning have many side effects like the corrosive properties that caused by some gases like $\mathrm{SO}_{2}$ and $\mathrm{H}_{2} \mathrm{~S}$ [97]. Among the fuels that got many attentions is the $n$-heptane, the low levels of particulates, carbon, sulfur oxides, and nitrogen oxides are the features of this fuel combustion [98]. In addition, $n$-Heptane was superior to natural gas fuel in energy releasing rate and it has lower water absorption, better miscibility with other fuels [99]. Dimethyl ether (DME) is introduced in many studies and researches. Arcoumanis et al [100] in their study concluded that DME can replace liquefied petroleum gas (LPG), diesel fuel, and liquefied natural gas (LNG). In addition, the variety of the sources that can produce DME like coal, natural gas, and biomass give this fuel the specialty in many applications [101]. 


\subsubsection{Fuel mechanisms}

The nature of the $\mathrm{HCCl}$ combustion that depends potentially on the species reactions within the combustion chamber can be affected dramatically by the applied kinetics mechanism. In addition, the complex nature of the chemical kinetics models is one of the main computing problems in combustion simulation. In many cases, the aim of the study will focus on a certain species, however, the species reaction will account for many other species within the fuel mechanism. Such accounting will increase the calculation cost typically. Radicals for example are very reactive species; they are usually presented in low concentrations. Such concentrations can play important role as intermediaries in the fuel reactions.

The elementary reactions that occur between the species can be ether fast or slow reactions. Those reactions define the fuel mechanism; a simplified chemical kinetics model requires the derivation of the simplest reactions system. Reduced mechanisms focus on the system parameters ether the global or the local ones. Global parameters like ignition delay and flame speed are of major concern; in the other side, parameters like the species concentration and the sensitivity rate coefficients are among the local parameters [102]. The reduced reactions system must be able to retain the essential features of the full original one.

One of the conventional techniques in mechanisms reduction is the steady state approximation technique $[103,104]$. This technique treats the appropriate radicals within the full mechanism so the partial equilibrium state will be achieved potentially due the fast reversible reactions. In the other side, the very slow reactions will be ignored in most cases. The identifying of the appropriate radicals requires deep knowledge in evaluating the species reactions. Defining the species reactions between fast elementary reactions and the very slow ones is the key in radicals' treatment. Order of magnitude approach can be used to estimate such reactions speeds by using information that depends on detailed reactions mechanisms data. In most cases, the results obtained by mechanisms reduction are expected to be valid only at specific ranges and conditions that belong to the original mechanism ranges.

The reactions in reduced mechanisms are not the same as the elementary mechanisms; they actually represent groups of the detailed reactions that lumped together to a simpler form. For the study case, this will make the reduced model more accurate within a specified area; however, the physical meaning of the elementary reactions will be missing. Reduced mechanisms can undergo a sensitivity analysis to obtain information that develops the mechanism. Finding the effective parameters that control the rate of the chemical reactions is the objective of using this type of analysis. Sensitivity analysis concern about determining the species concentration variations within the system, for example, if the reaction is slow and unimportant, the system will not be sensitive to it. In the other side, when the reaction is very fast, the system will be affected simultaneously as the reaction is occurred. In some cases, the sensitivity analysis may not capture some of the important fast reactions, so less accurate mechanisms will be resulted [105, 106, 107].

Many studies were performed to investigate the development of specific reduced mechanisms for describing the $\mathrm{HCCl}$ combustion specifically. For example, Kim et al [108] developed a new reduced chemical kinetics mechanism that contains 45 reactions and 28 species for DME combustion. They defined the mechanism based on a given initial conditions like, the fuel-air mixture concentration, temperature and pressure. The mechanism was used to predict those conditions as a function of the time. In their results, they compared the measured data and the detailed mechanism to validate the new mechanism. A good agreement was obtained when validating the initial pressure and peak temperature of the reduced 
mechanism. Another successful iso-Octane combustion mechanism was presented by Aceves et al [109]. In their mechanism, a fast and accurate prediction of the ignition timing in an $\mathrm{HCCl}$ engine was the objective. The reduction process was done by reducing a detailed mechanism that contains 859 species and 3606 reactions to one that has 199 species and 383 reactions. The two mechanisms were implemented in a numerical simulation to compare some of the combustion features. The results indicated high similarity in the pressure traces and the HRR between the reduced and the detailed mechanisms. However, the calculated $\mathrm{CO}$ and UHC emissions by the reduced mechanism were different than those predicted using the detailed mechanism. The reason was referred to the fact that the reduced mechanism was developed for $\mathrm{HCCl}$ ignition predicting purposes only not for UHC and CO emissions investigations. From computational time perception, the reduced mechanism required much less time that could be as 2 hours vs. 2 days.

\subsection{Chemical kinetics approach}

\subsubsection{Chemical kinetics}

The science of the chemical kinetics interests in tracking hundreds of elements species and thousands of their reactions [6]. Although chemical kinetics models are known since decades, but there are many developments which happened in this field like introducing chemical kinetics in many CFD simulations [6, 7]. On the other hand, reducing the computational demands of the reactions is satisfied when a proper managing and evaluation of the species reactions is considered [7].

\subsubsection{Species chemical reactions:}

The series of equations that describe the bond-making and bond-breaking steps that occurs during the conversion of reactants to products are summarized within the fuel mechanisms [6]. These mechanisms were found based on many factors. The number of the species, which is involved, the number of the possible reactions, the effective reactions that were considered and those that had been neglected are among those factors [7].

All models used in combustion chemistry share the same elementary kinetics reaction description, where an Arrhenius law represented that, the rate of those reactions inside the mechanism was expressed by the rate coefficient as [110]:

$k=A T^{\beta^{\frac{-T T_{a}}{T}}}$

The values of $A, T_{a}$ and the temperature dependence coefficient $\beta$ are completely dependent on the reaction nature. The rate coefficient defines different levels of approximation to describe the kinetics [110]. A good example is to look on a three mechanisms of Methane $\left(\mathrm{CH}_{4}\right)$ as following.

- The Global one-step reaction mechanism of Duterque [111]:

$$
\mathrm{CH}_{4}+2 \mathrm{O}_{2} \longrightarrow \mathrm{CO}_{2}+2 \mathrm{H}_{2} \mathrm{O} \quad \mathrm{A}=1.5 \mathrm{E} 13, \beta=0.0, \mathrm{~T}_{\mathrm{a}}=20,000 \text { (K) }
$$

- The four- steps reaction mechanism of Joens and Lindstedt [112]:

$$
\begin{array}{lll}
\mathrm{CH}_{4}+2 \mathrm{O}_{2} & \longrightarrow \mathrm{CO}_{2}+2 \mathrm{H}_{2} \mathrm{O} & \mathrm{A}=4.40 \mathrm{E} 14, \beta=0.0, \mathrm{~T}_{\mathrm{a}}=30,000(\mathrm{~K}) \\
\mathrm{CH}_{4}+\mathrm{H}_{2} \mathrm{O} & \longrightarrow \mathrm{CO}_{3} \mathrm{H}_{2} & \mathrm{~A}=3.00 \mathrm{E} 11, \beta=0.0, \mathrm{~T}_{\mathrm{a}}=30,000(\mathrm{~K}) \\
\mathrm{H}_{2}+0.5 \mathrm{O}_{2} & \longrightarrow \mathrm{H}_{2} \mathrm{O} & \mathrm{A}=2.50 \mathrm{E} 19, \beta=0.0, \mathrm{~T}_{\mathrm{a}}=40,000(\mathrm{~K}) \\
\mathrm{CO}+\mathrm{H}_{2} \mathrm{O} & \longrightarrow \mathrm{CO}_{2}+\mathrm{H}_{2} & \mathrm{~A}=2.75 \mathrm{E} 12, \beta=0.0, \mathrm{~T}_{\mathrm{a}}=20,000(\mathrm{~K})
\end{array}
$$

- The standard detailed mechanism GRI3.0 (53 species, 325 reactions) [113]. 


\subsubsection{Combustion chemistry:}

The chemistry of combustion has a very important rule in treating environmental problems. Emissions can be reduced if the nature of their producing reactions is defined correctly [7]. For example, $\mathrm{NO}_{\mathrm{x}}$ emissions are one of those emissions; it can be effectively reduced by using $\mathrm{HCCl}$ combustion technique [2].

The combustion in internal combustion engines is defined by the chemical kinetics knowledge of the species reactions. Many of the combustion events features can be investigated based on the well understanding of the chemical kinetics approach [7].

\subsection{Ignition delay:}

The time needed by the air-fuel mixture to be ignite is called the Ignition delay [6]. In $\mathrm{HCCl}$ engines, the ignition process occurs under the influence of the increasing pressure and the temperature of the mixture. This fact makes the chemical kinetics as the dominant in determining the process ignition time [2]. The ignition delay is divided into two main periods, the time since the start of air-fuel mixing until the attainment of chemical reaction conditions is called the physical delay [114]. Fuel atomization, vaporization and mixing with air occur during this time. The viscosity of the fuel mixture controls this time, such for low viscosity fuels, the physical delay is small and vice versa. The second period is the chemical delay, which covers the slow starting of the reactions and the acceleration of these reactions until inflammation or ignition is happened [114]. In general, chemical delay is longer than the physical delay; however, the temperature of the surroundings has a strong effect on this time. Chemical reactions are faster at higher temperatures thus physical delay becomes longer than the chemical delay in such cases [114].

\subsection{Combustion duration:}

The whole process from the initiation to the termination of the combustion reactions is defined as the combustion duration. Two major reactions groups are included within this period, the chain propagating reactions and the chain branching reactions. Within these groups, different types of radical's processes are happening and once these processes reached the equilibrium state, the combustion will end [115].

\subsubsection{Species governing equations:}

\subsubsection{Species production rate:}

The forward and backward stoichiometric reactions of $K_{t h}$ number of species is described by the following equation [110]:

$\sum_{k=1}^{K} v^{\prime}{ }_{k i} \chi_{k} \leftrightarrow \sum_{k=1}^{K} v^{\prime \prime}{ }_{k i} \chi_{k} \quad(i=1, \ldots \ldots, I)$

Where $\mathrm{K}$ is the number of species involve in the reactions, $v$ is the stoichiometric coefficients, $i$ is the number of reactions covered by the mechanism, $\chi$ is the chemical symbol for the specie and the superscripts (') indicates forward reaction while (") refers to backward one.

The production rate $\dot{\omega}_{k}$ of the $\mathrm{k}_{\text {th }}$ species is the total summation of the rate of the progress for those reactions that involve the species [110].

$$
\dot{\omega}_{k}=\sum_{i=1}^{I} v_{k i} q_{i}
$$


Where $v_{k i}=v^{\prime \prime}{ }_{k i}-v^{\prime}{ }_{k i}$ and $q_{i}$ is the rate of progress variable for the $\mathrm{i}_{\text {th }}$ reaction which is given by the difference between the forward and backward reactions rates [110]. The rate of the progress is illustrated by equation (1.17).

$$
q_{i}=k_{f i} \prod_{k=1}^{K}\left[X_{k}\right]^{v^{\prime} k i}-k_{b i} \prod_{k=1}^{K}\left[X_{k}\right]^{v \prime^{\prime} \mathrm{k} i}
$$

Where $X_{k}$ is the molar concentration of the $k_{\text {th }}$ species, $k_{f i}$ and $k_{b i}$ are the forward and backward rate constants of the $i_{\text {th }}$ reaction given by equation (1.17).

\subsection{Species mass conservation:}

Assuming the reactor in Figure 6, the mass conservation of the species within the controlled boundary of the reactor is following equation (1.20) [110].

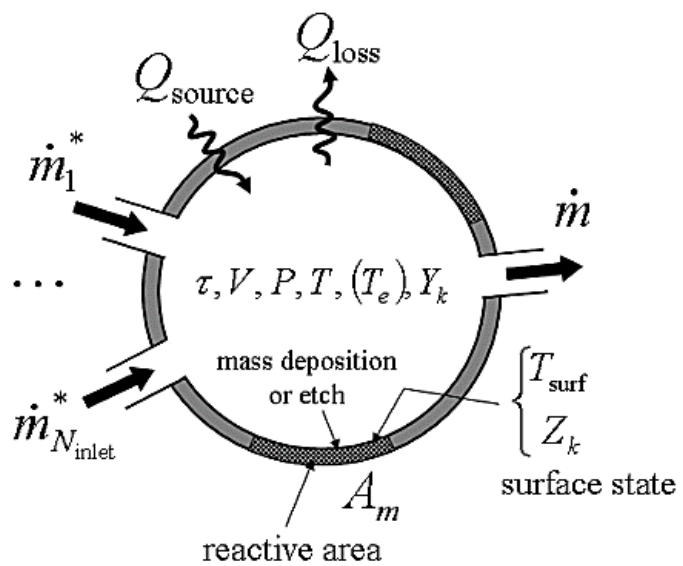

Figure 6 Schematic Representation of a Well Mixed Reactor Module [110].

$\left(\rho_{k} V_{r}\right)^{j} \frac{d Y_{k}^{j}}{d t}=\sum_{i=1}^{N_{\text {inlet }}^{j}}\left(\dot{m}_{i}^{j}\right)_{\text {inlet }}\left(Y_{k . i}-Y_{k}\right)_{\text {inlet }}+\sum_{i=1}^{N_{P S R}{ }^{j}}\left(\dot{m}_{k}^{r}\right)_{\text {outlet }} R_{r j}\left(Y_{k}^{r}-\right.$

$\left.Y_{k}^{j}\right)_{\text {outlet }}-Y_{k}^{j} \sum_{m=1}^{M} A_{m}^{j} \sum_{k=1}^{K_{g}} \dot{s}_{k, m}^{j} W_{k}+\left(\dot{\omega}_{k} V\right)^{j} W_{k}+\sum_{m=1}^{M} A_{m}^{j} \dot{s}_{k, m}^{j} W_{k}$

Here $\mathrm{j}$ is the reactor number, $\rho$ is the mass density, $\mathrm{V}_{\mathrm{r}}$ is the reactor volume, $\dot{m}_{\text {inlet }}$ is the inlet mass flow rate, and $\dot{m}$ is the outlet mass flow rate. $N_{\text {inlet }}{ }^{j}$ is the number of inlets for each reactor $\mathrm{j}$, while $N_{P S R}{ }^{j}$ is total number of the reactor modules in the reactor network. $R_{r j}$ is the fraction of the outflow of the reactor $\mathrm{j} . A_{m}^{j}$ is the surface production rate of the $\mathrm{k}_{\mathrm{th}}$ species on $\mathrm{m}_{\mathrm{th}}$ material per unit surface area. $Y_{k}{ }^{j}$ is the mass fraction of the $\mathrm{k}_{\mathrm{th}}$ species. $W_{k}$ is the molecular weight of the $\mathrm{k}_{\mathrm{th}}$ species, $\dot{\omega}_{k}$ is the molar rate of production of the $k_{\mathrm{th}}$ species by the gas-phase chemical reaction per unit volume, and finally $\dot{s}_{k, m}^{j}$ is surface reaction rate [110].

If no-inlet and no-outlet are considered, the reactor will be an internal combustion device (IC). Equation (1.20) will reduced to [110]:

$$
\frac{d Y_{k}^{j}}{d t}=\frac{\dot{\omega}_{k}^{j} W_{k}}{\rho}
$$

\subsection{Species energy conservation:}

The energy conservation of the species is described in equation (1.22) [110]. 


$$
\begin{gathered}
\frac{d U_{\text {sys }}^{j}}{d t}=\sum_{i=1}^{N_{\text {inlet }}^{j}}\left(\dot{m}_{i}^{j}\right)_{\text {inlet }} \sum_{k=1}^{K_{g}}\left(Y_{k, i} h_{k, i}\right)_{\text {inlet }}+\sum_{i=1}^{N_{\text {inlet }}{ }^{j}} m^{r}{ }_{\text {outlet }} R_{r j} \sum_{k=1}^{K_{g}}\left(Y_{k} h_{k}\right)^{r}- \\
\left(\sum_{k=1}^{K_{g}} Y_{k} h_{k}\right)^{j}-Q_{\text {loss }}^{j}+Q_{\text {source }}^{j}-P^{j} \frac{d V^{j}}{d t} \quad j=1, N_{P S R}
\end{gathered}
$$

$U_{\text {sys }}$ represents the total internal energy of the system including, the internal energy of the gas, the gas surface phase and deposited solid phase, $Q_{\text {loss }}$ is the net heat flux directed out of the reactors. Many assumptions can be considered for modeling the heat loss within the reactor. In general, the heat loss is calculated based on equation (1.21) [110]:

$Q_{\text {loss }}=A h_{t}\left(T-T_{0}\right)$

$Q_{\text {source }}$ in equation (1.21) refers to the energy deposited into the gas inside the reactor. The term $\mathrm{P}(\mathrm{dV} / \mathrm{dt})$ is the work done by the control volume and $h$ is the enthalpy for the $k_{\text {th }}$ species at each reaction $i$. When a close system reactor is assumed, the energy equation (1.22) will reduced to equation (1.24) [110]:

$\frac{d U_{\text {sys }}^{j}}{d t}=-P^{j} \frac{d V^{j}}{d t}-Q_{\text {loss }}^{j}+Q_{\text {source }}^{j}$

Or in temperature form as:

$\rho C_{p} \frac{d T^{j}}{d t}=\frac{d P^{j}}{d t}-\left(\sum_{k=1}^{K_{g}} Y_{k} h_{\mathrm{k}}\right)^{j}$

\subsubsection{Chemical kinetics software:}

In order to simulate the combustion process, the governing equations, which were mentioned, need to be solved. The nature of species reactions equations is very stiff. This refer to the fact that, the combustion process is very rapid and random process. To solve the combustion reaction, regular methods like Runge-Kutta will come out with inaccurate results or it may not work at all [2]. However, MATLAB ${ }^{\circledR}$ ordinary differential equation (ODE) solvers like ode15s and ode23s have the ability to solve chemical kinetics problems. The algorithms that were used in those solvers were set based on a variable time steps numerical methods like Gear's method [116]. Many software packages can be used in order to feed these solvers with the required reactions rate equations, the thermodynamic and transport properties [117]. Chemkin (a Chemical kinetics software) for example, it has a huge database which describes thousands of reactions for many fuel types [110]. In this research, Cantera (a toolbox from California institute of technology) will be used; it has almost the same features of $\mathrm{CHEMKIN}^{\circledR}$ but with more flexibility in simulating special types of engines like the free position engine [117]. 


\section{Chapter 2. Research objectives and approach}

\subsection{Research objectives}

The research objectives have been set based on the belief that chemical kinetics approach has many advantages over the empirical one in terms of the accuracy. In addition, the fact that chemical kinetics can provide a better estimate of the actual $\mathrm{HCCl}$ combustion process is another motivator of the study. To make the study more specific, a model with a detailed geometry is used to investigate the following:

- The HRR effect on the performance of the free-piston engine.

This objective was set because of the importance of the HRR in defining the combustion output pressure that affect the piston dynamics.

- The nature of the relation between the dynamics and the thermodynamics of the free piston engine.

This objective is an extension for the first one. Piston dynamics and the in-cylinder process affect each other's indirectly, based on that, good investigations of the piston dynamics could help in achieving a better understanding of the HRR role.

- The system parameters effects on the engine performance.

The aim in this objective was to study the different system parameters and define the most effective ones. This helped not only in optimizing the engine outcomes, but it defined the engine capabilities and limitations as well.

- The possibility of establishing a realistic and efficient controlling system.

This objective considered the fact that $\mathrm{HCCl}$ combustion is a difficult process to be controlled. In addition to the challenge that obtained by the unconstrained geometry of the free-piston engine. Therefore, the controllers were designed to eliminate the cyclic variations in the free-piston engine operation with the maximum possible effectiveness.

\subsection{Research body}

The study covers three sections, the simulation, the parametric study and the model control.

$>$ Numerical simulation:

In this research project, all the tasks have been performed using numerical analysis. MATLAB ${ }^{\circledR}$ is the main platform side by side with Cantera toolbox. Cantera algorithms for species reactions and their thermodynamics properties feeds the MATLAB ${ }^{\circledR}$ main code. The general solution of system sub-models has been performed and verified with Chemkin ${ }^{\circledR}$ results. This step is required to ensure that the calculated HRR values are consistent.

At first, a fuel has been defined using Cantera by defining its mechanism. The mechanism includes all of the participating species and reactions for the fuel. Basic hydrocarbon's fuels like Methane $\left(\mathrm{CH}_{4}\right)$, is the main concern of this study. The huge available data for those fuels gives the flexibility to validate the results with those in the literature. Thereafter, all of the state conditions before the combustion have been defined. Initial pressure and temperature, air to fuel ratio (AF) and percentage of exhaust gas recirculation (EGR) are examples for state definition parameters. Then, a set of equations consists of the first law of thermodynamics, the ideal gas law and piston dynamics carry out the fuel-air mixture within a multi-processes path. The mixture follows four main stages that are compression, combustion, expansion and scavenging. Finally, a combustion model represented by Cantera equations of reactions has been solved using an ODE solver in the $\mathrm{MATLAB}^{\circledR}$ main code. 


\section{$>$ Parametric study:}

Once the numerical simulation results are available and validated, a parametric analysis can be done. The effect of the different parameters on the system performance is the target of this task. Variable equivalence ratio is one of those important variables. The fuel-air mixture can be defined as lean, rich or stoichiometric based on the value of the equivalence ratio. Testing different states of equivalence ratio helps to identify the optimal zones of the used free-piston engine model. EGR is also an important parameter. It changes the fuel-air percentage (when constant mixture mass is assumed) by adding some of the combustion products to the fresh charge.

This section of the study interests also in the sensitivity analysis of some parameters like fuel kinds and mechanisms. Mechanism reduction for example is one of the good tasks that has been investigated within sensitivity analysis part. The reduction in the fuel mechanism refers the concepts of considering the main species reactions of the fuel, therefore; less number of species reactions that involve in the combustion process. In the case of the fuels, the performance of the engine has been tested using different types of fuels, In addition to fuels with different compositions like the natural gas.

$>$ Model control:

In this part of the research, different controlling techniques have been applied using the system parameters with the most impact on the performance. The controller is one of the main parts of the system; it works based on input-output algorithm. The equilibrium between the desired values and the feedbacks of the controller defines the steady state operation. The steady state operation has been defined in this study based on the nature of the required results. For example, in the case of the multicycle simulation, the near steady state conditions have been reached with the minimal possible cyclic variations. This gives the ability to the results to be validated and to be more realistic.

\subsection{Research approach}

To summarize the study procedure, many tasks have been set and treated in order to satisfy the research objectives.

1. Task 1: Building the engine models

In this task, both of the dynamic and thermal sub-models of the engine have been established. The main dynamic sub-model treats the different loads that affect the system. Generator, springs and friction loads represent some of those loads. Generator is considered the monitor that introduces the engine performance; it has a significant role in the controlling part as well. The dynamic model is covered by Newton's second law, where a forces balance is done over the engine's piston, electrical, frictional, potential and pressure forces define the piston motion with this law. The pressure force is the point of concern since it will be the major thermodynamic sub-model output.

The in-cylinder thermodynamics sub-model is the core of this research. It has the direct relation to the chemical kinetics approach. Represented by $\mathrm{HCCl}$ combustion, the chemical kinetics model has been built and coupled with the dynamic one. Cantera is the platform of this model; it includes the fuel species database in addition to the governing equations for those species reactions. Thereafter, a set of ordinary differential equations (ODEs) have been written based on the first law of thermodynamics and the ideal gas law. Solving this set of ODEs requires an advanced ODEs solver because of the stiffness of the chemical kinetics reactions. The fact that fuel combustion is very rapid and fast process makes the solving of reactions a very stiff process. Stiff equations solver has been used in order to solve these equations for 
the pressure variation over the engine piston. The thermodynamic sub-model feeds the dynamic one with the required pressure force term. However, a heat transfer model has been also coupled with the incylinder thermodynamics by using a modified formula for Woschni. The formula considers the $\mathrm{HCCl}$ nature and it has been used to demonstrate the effect of losing energy through the engine cylinder.

For both of the dynamic and thermodynamic sub-models many sets of parameters have been defined. The engine geometry, the spring stiffness and the load profile are examples of the dynamic parameters. On the other hand, fuel composition, intake conditions, equivalence ratio and EGR percentage are thermodynamic parameters.

The results of this task follows two paths, a cycle-to-cycle simulation which is the main part of this task and secondly, a basic parametric study. In the cycle-to- cycle simulation, the variation between the engine's cycles have been tested and treated using a proper controller. Model control, which is one of the objectives is considered as the master key in obtaining system stability and eliminating the cyclic variations. At the same time, stable single engine's cycle has been employed for testing the effects of the different model parameters.

2. Task 2: Model Parametric Study

Beyond task 1, a comprehensive parametric study has been performed to gain a full understanding of the effects of the model parameters on the system performance. Many parameters have been tested under this task. Engine geometries, translator mass and spring stiffness profile have been examined as dynamic parameters, however, Intake pressure, inlet temperature, equivalence ratio and EGR have been evaluated as in-cylinder thermodynamic parameters. The parameters effects is monitored using many outputs like system efficiency and stability.

3. Task 3: Results verification

The model results have been verified and compared to results from different articles, a comparison between the system outputs has been applied especially in those belong to the combustion process. Numerical data has been used to validate the model results.

4. Task 4: Emissions analysis

In this task, the behavior of the system from emissions perception has been investigated. For example, using $\mathrm{HCCl}$ is a good chance to investigate $\mathrm{NO}_{\mathrm{x}}$ emissions where $\mathrm{HCCl}$ combustion has low levels of them.

5. Task 5: Model control and optimization

The variations in the piston operation should be minimized in order to achieve a stable system performance, at the same time; the controller must consider the maximum system efficiency. In the current research, the electrical generator plays a significant role in controlling the engine. The variations in the $\mathrm{CR}$ and the BDC are the used within the presented controllers. To satisfy the different engine loads, the alternator has been set in the core of the controlling the system operation. The dynamic load profile of the alternator has the potential to neutralize any disturbances that caused by the other system forces. The effectiveness of the controller is satisfied when both of the minimum cyclic variations and maximum efficiency are satisfied. 


\section{Chapter 3. Free-piston and chemical kinetics approach}

\subsection{Free piston engine cycle processes}

The cycle of the free piston engine follows four main processes:

- Compression of the intake charge up to the ignition point.

- Combustion of the fuel-air mixture, which define the HRR.

- Expansion of the combustion products.

- Scavenging or combustion products blowdown.

Two different approaches can be followed to model the previous processes. The first one is by defining the four different process individually. In this approach, an assumed isentropic or polytropic process along the compression and the expansion stroke is used. The HRR of combustion is defined by an empirical correlation like the Wiebe function. However, the first three processes will be fully coupled within the second approach. Chapter one equations (1.20), (1.21), (1.22) in addition to the heat release rate are solved and calculated by the chemical kinetics model. This approach is more complicated but it has results that are more accurate.

\subsubsection{Empirical based approach}

In this approach, the pressure and temperature are related to the piston position (volume) by the specific ratio $\gamma={ }_{p} / C_{v}$ as following:

$\frac{P_{2}}{P_{1}}=\left(\frac{X_{1}}{X_{2}}\right)^{m}$

$\frac{T_{2}}{T_{1}}=\left(\frac{X_{1}}{X_{2}}\right)^{m-1}$

Where $P_{2}, T_{2}, V_{2}, P_{1}, T_{1}$, and $V_{1}$ are the pressures, temperatures and volumes at the end and at the start of the compression or of the expansion process respectively. Typically, $\mathrm{m}$ is the polytropic constant and it can be equal to a certain value $(\gamma)$ for a perfect isentropic process. When less value is introduced, this mean that losses are included in the process [6].

To evaluate the HRR, an empirical formula can be used. Wiebe functions are the most well-known formulas in this field. They have the ability to include some other effects like the walls temperature. A double Wiebe function is assumed in those cases. An example of Wiebe function is illustrated in equation (3.3) [23].

$\frac{d Q_{c}}{d t}=a \cdot \frac{b+1}{c d} \cdot\left(\frac{t-t_{s}}{c d}\right)^{b} e^{\left(-a \cdot\left(\frac{t-t_{s}}{c d}\right)^{b+1}\right)} \cdot Q_{c o m b}$

Where $d_{c} / d t$ is the HRR rate of fuel combustion, $Q_{\text {comb }}$ is heating value of the fuel-air mixture, $C d$ is the combustion duration, $t_{s}$ is the combustion starting time and $a$ and $b$ are the functional shape parameters. The heat release rate is solved for pressure at first. The piston location then will be evaluated and used in the next cycle.

In order to initiate the combustion, a correlation for the ignition delay is used to find the time needs to start the combustion. Many methods are used to find this period, one of those methods is the knockintegral method (KIM). The correlation was derived using rapid compression testing machines output data. KIM empirical formula has the following form [118]: 
$\tau=A e^{(b / T) P^{n}}$

Where $\tau$ is the ignition time delay, $\mathrm{T}$ and $\mathrm{P}$ are the fuel-air mixture temperature and pressure and $\mathrm{A}, \mathrm{b}$ and $\mathrm{n}$ are empirical constants which determined for each engine.

The combustion products need to be equalized after the expansion process. This process is called scavenging or exhaust gas blowdown. The simplest approach to model the scavenging as a perfect gases blowdown. This approach assumes that all of the combustion products are replaced by a new fresh fuelair charge at each cycle.

Atkinson, et al. [23] for example used this approach to model a free-piston engine operation. The P-V diagram of their model is represented in Figure 7. The combustion process is not identical, instead it follows the compression trend smoothly. Basically, the assumed Wiebe function influence this trend of the HRR. The Wiebe function parameters were adjusted by matching the function profile to an experimental date set. The scavenging in this model was assumed to be perfect.

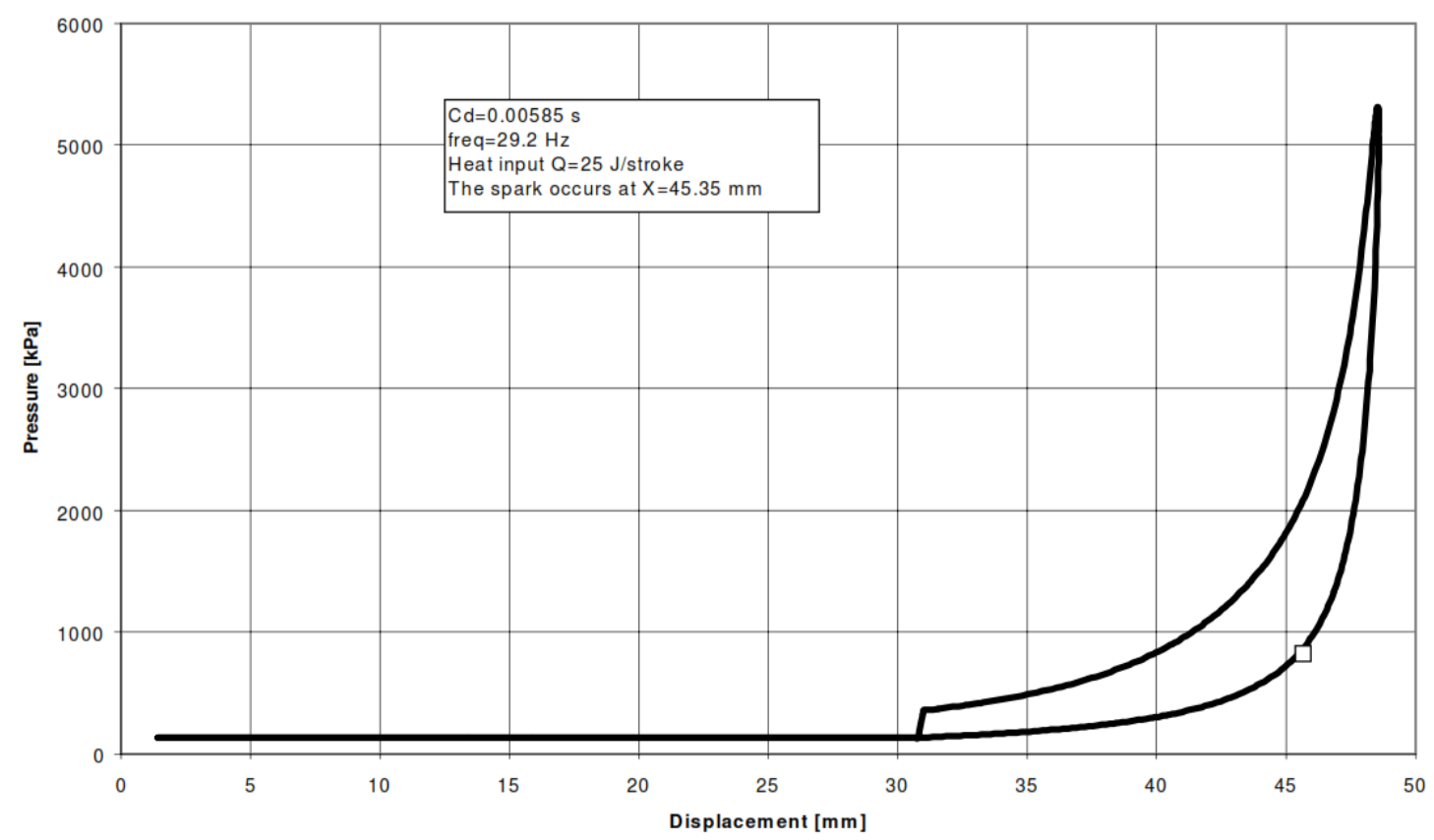

Figure 7 In-Cylinder Pressure vs. Piston Assembly Displacement [23].

Figure 8 shows the difference between the velocity profiles of the free piston engine and the conventional one. The free piston has elongated and semi-rectangle profile while the regular engine profile has semi-egg shape. The fact that free piston engines have no crankshaft explain this difference since no geometrical restrictions are applied on the piston motion during the compression and the expansion strokes [51]. 


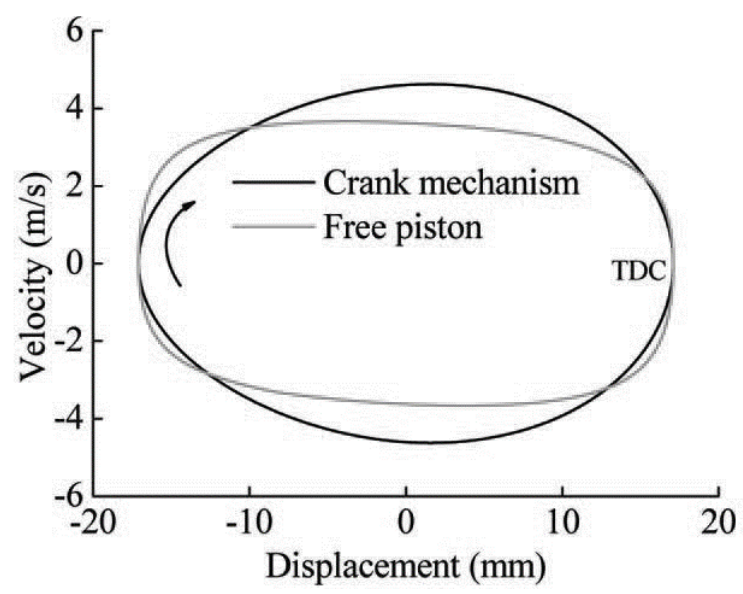

Figure 8 Crankshaft Engine vs. Free Piston Engine cycle [51].

\subsubsection{Chemical kinetics approach}

Chemical kinetics models have the ability to simulate the compression, combustion and expansion processes at the same time. The first law of thermodynamics and the ideal gas laws, in addition to the combustion rate equations are coupled within the model solver. The solution of this set will result with a unique cycle profile. The matching point between the piston dynamics and the mixture chemical kinetics is the HRR of the burning fuel. The combustion process defines the pressure force, which is one of the most important acting forces over the piston. This force causes the piston to move during the expansion stroke due to the sudden and rapid increase in the in-cylinder pressure. In the other side, the piston will store energy as well as the springs that supports the compression stroke in the next cycle.

Species reaction equations are very stiff and sensitive since a specific solver is required for this purpose. On the other hand, the nature of the species reactions depends on the type of the mechanism that is used to describe the species reactions. Full or reduced mechanisms can be used depending on the results sensitivity and the computational cost, which is available.

Fortunately, species mechanisms and reactions are available within many software packages as long as their thermodynamic properties. $\mathrm{CHEMKIN}^{\circledR}, \mathrm{KIVA}^{\circledR}$ and Cantera are examples of those Software, they have a full data set for many types of fuels. Some of platforms like CHEMKIN ${ }^{\circledR}$ has its own interface and solvers. Cantera has been employed in this research with a MATLAB ${ }^{\circledR}$ ODE solver code. The solver has been coupled with the dynamic sub-model in order to complete the simulation loop.

Goldsborough and Van Blarigan [18] used HCT (mentioned before) to simulate the species reactions in a free piston engine using zero-dimensional approach. The modeling procedure in their work was described in the literature review, section 1.3. The P-V diagram of their engine is shown in Figure 9. The four main processes are clearly shown. The combustion process is represented as a step jump in pressure at nearly constant volume, while the compression and expansion processes are identically following the same trend. 


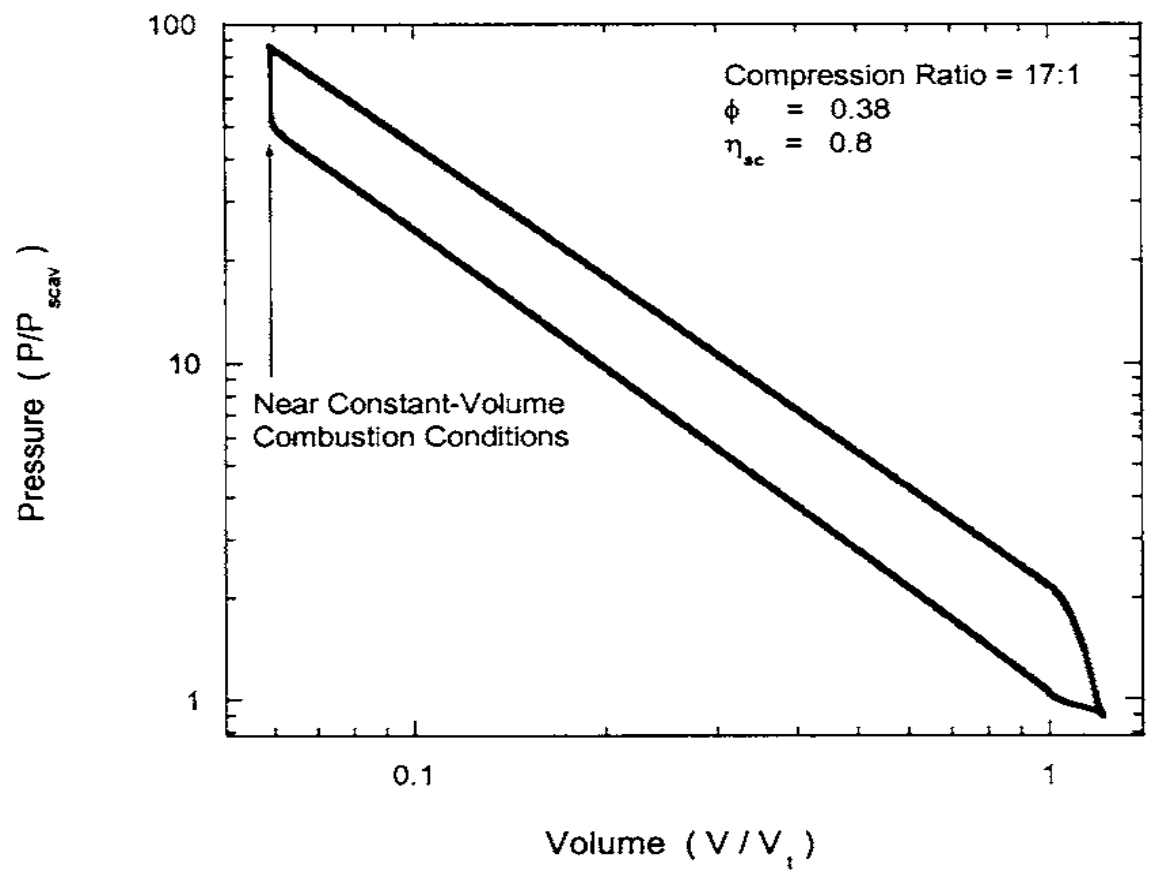

Figure 9 P-V diagram for a free piston engine using HCT [18].

Figure 10 shows the combustion event in Goldsborough and Van Blarigan [18] engine. The process is rapid and fast and it looks like a step or jump in the pressure near the TDC. Figure 10 explains the difference between the two different HRR approaches since the combustion event is identical.

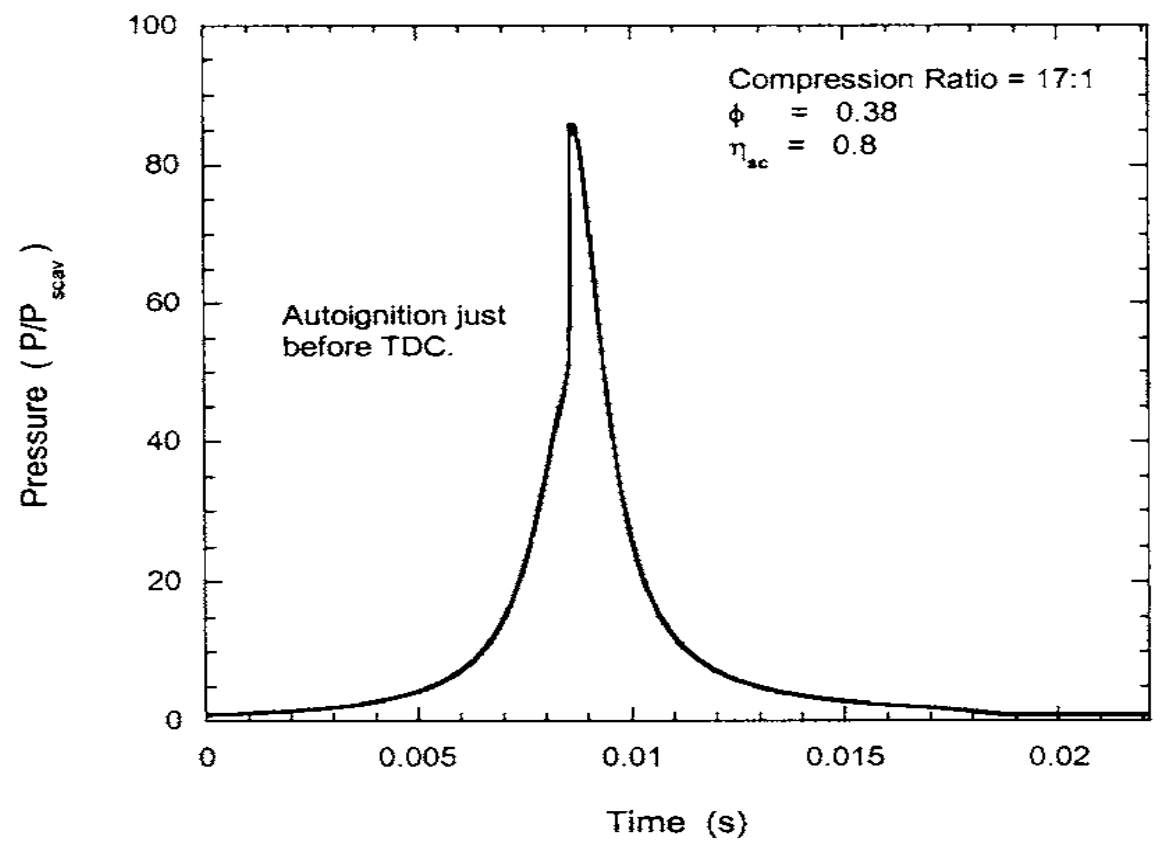

Figure 10 Cycle pressure including the combustion event using HCT [18]. 
The products blowdown process was assumed to follow an S-shaped correlation derived by Sher and Harari's [119]. The scavenging efficiency is given in equations (3.5-3.7):

$$
\begin{aligned}
& \eta_{s}=\left(\frac{T}{T_{0}}\right) \eta_{c} \\
& \eta_{c}=\lambda-\int_{0}^{t} \frac{\beta \lambda}{\beta+(1-\beta)^{T} T_{T_{i}}} d t \\
& \beta=1-e^{-\gamma \lambda t_{s}^{\delta}} \\
& t_{s}=\frac{t-t_{s o}}{t_{s c}-t_{s o}}
\end{aligned}
$$

Where $\eta_{s}$ and $\eta_{c}$ are the scavenging and the charging efficiencies, $\beta$ is the S-type exponential factor, $T, T_{0}$ ,$T_{b}$ and $T_{i}$ are the instantaneous, ambient, burnt gas and fresh charge temperatures respectively. However, $\delta$ and $\Upsilon$ are shape factors with almost values of 2.0 and 1.7 respectively; $\lambda$ is the delivery ratio and equal to the mass delivered of charge to the multiplication of the ambient density by the displaced volume. $t_{s}$ is the non-dimensional time based on the scavenging port closed (sc) and opened (so). For simplicity, the scavenging has been assumed as a perfect process in this study.

\subsection{Model analysis and multi cycles simulation}

The model describes the processes in a single cylinder, two stroke free piston engine, which is opposed by a mechanical spring. Two main sub-models are introduced in order to simulate the engine performance, the dynamic engine sub-model and the in-cylinder thermodynamic sub-model. The current engine schematic is illustrated in Figure 11 (a) and (b). The schematic shows the engine components and force balance on the translator. The compression spring acts to return the translator after cylinder expansion to complete the engine cycle. Previous investigations by researchers at WVU have explored the effects of springs within a free piston engine device [120]. The springs or some other rebound mechanism such as a bounce chamber (i.e. air spring), are necessary for the single cylinder architecture, but can also be used to tailor the motion of the translator. In Figure 11(b), the dynamic model and the in-cylinder thermodynamic model are described according to the forces and thermodynamic terms important to the system. Additional input parameters for the engine model are summarized in Table 1. 


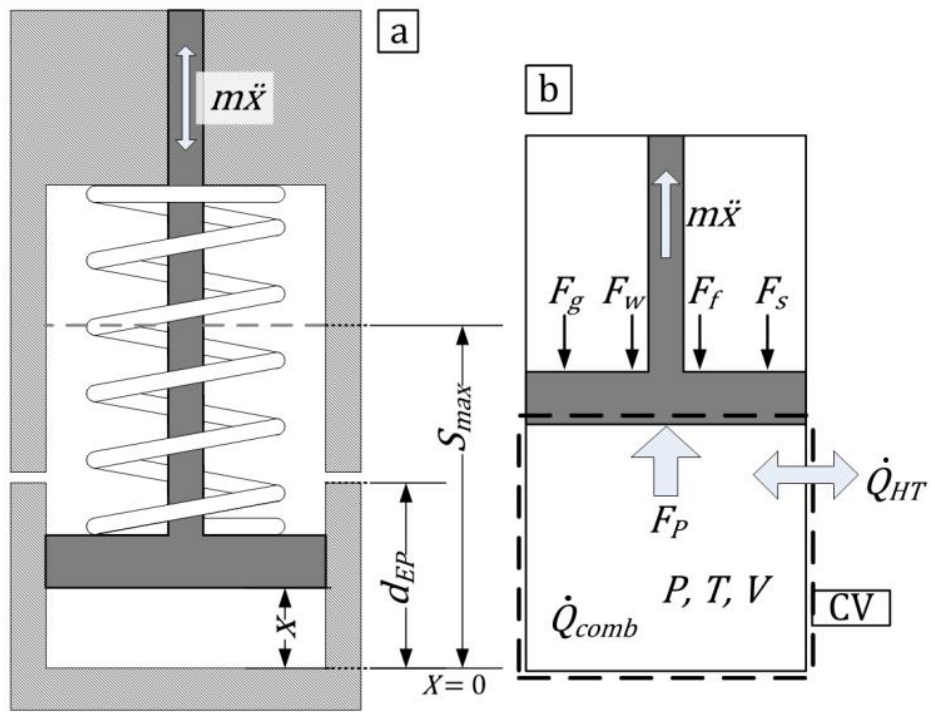

Figure 11 (a) Free piston engine schematic, (b) Dynamic and thermodynamic model diagram.

Table 1 Parameters used in the simulation

\begin{tabular}{lcc}
\hline Parameter & Symbol & Value \\
\hline Bore & $b$ & $30 \mathrm{~mm}$ \\
Maximum stroke & $S_{\max }$ & $51 \mathrm{~mm}$ \\
Distance to port & $d_{E P}$ & $35.7 \mathrm{~mm}$ \\
Moving mass & $m$ & $0.5 \mathrm{Kg}$ \\
Intake temperature & $T_{i n}$ & $600 \mathrm{~K}$ \\
Intake pressure & $P_{\text {in }}$ & $100 \mathrm{Kpa}$ \\
Spring stiffness & $k$ & $232.5 \mathrm{~N} / \mathrm{m}$ \\
Spring zero force location & $x_{S}$ & $0.018 \mathrm{~m}$ \\
Fuel (CH4[80\%], C2H6[11\%], C3H8[9\%]) & - & $0.00084(\mathrm{~g})$ \\
Air (N2 [79\%], 02[21\%]) & - & $0.01440(\mathrm{~g})$ \\
\hline
\end{tabular}

\subsection{Dynamic sub-model}

In the dynamic sub-model, the translator acceleration, velocity, and position are obtained from the balance of the forces acting on the translator. These include the total friction force $\left(F_{f}\right)$, spring force $\left(F_{s}\right)$, force due to gravity $\left(F_{g}\right)$, the alternator load force $\left(F_{w}\right)$ and the cylinder pressure force $\left(F_{P}\right)$. The forces can be written in a dynamic equation using Newton`s second law as in equation (3.8):

$m \frac{d^{2} x}{d t^{2}}=F_{P}-F_{w}-F_{g}-F_{f}$ 
Where $m$ is the piston mass, $x$ is the piston position with the reference point shown in Figure 11 (a), and $t$ is the time. Each force is described in the following equations.

$F_{P}=A \cdot P$

$F_{g}=m \cdot g$

$F_{f}=C_{f} \cdot \sin \dot{x}$

$F_{S}=k \cdot\left(x-x_{s}\right)$

In equations (3.9) through (3.11), $P$ is the instantaneous in-cylinder pressure, $A$ is the piston area, $g$ is the acceleration due to gravity, $C_{f}$ is the friction coefficient. The spring force $\mathrm{F}_{\mathrm{s}}$ depends on its stiffness $(k)$ and its free length, zero force location $\left(x_{s}\right)$.

One of the main force in this sub-model is the alternator load. This load can be presented in different profiles in order to seek the steady state operation. Examinations of the effect of alternator load shape have been considered within this dissertation by applying different load profiles to control the system. The load profile could have the potential to obtain the steady state performance especially if it's shape fit with the other forces. However, deeper investigations can be performed in the future to check the performance of the engine model under different load profiles. In the meanwhile, a sinusoidal load has been considered within the basic simulations, the load force is shown in equation $(3.12)[40,41]$.

$F_{w}=-C_{A} \cdot\left(1-\cos \left(\frac{2 \pi}{s_{\max }} \cdot x\right)\right) \cdot \operatorname{sign}(\dot{x})$

In the above equation, $S_{\max }$ represents the maximum possible stroke length and $\mathrm{C}_{\mathrm{A}}$ is the alternator load coefficient.

The alternator load force is considered in this study because of the nature of this load that can play a significant role in controlling the performance. However, this research does not focus specifically on the load shapes, but instead it assumes one-half of a sinusoidal load profile. The sign of the velocity is applied to the profile to ensure the force opposes the motion of the translator. The shape of the load profile is dictated by the engine geometry and instantaneous translator position so that the load is zero at the positional boundaries ( 0 and $S_{\max }$ ) and achieves its peak force at the halfway point between the boundaries $\left(S_{\max } / 2\right)$. Later on this dissertation, another controller is discussed; the PI controller, which seeks to obtain a specific $C R$ value, considers the piston velocity as a main factor to achieve the steady operation.

A comparison between constant and variable loads can be found in Figure 12. The difference can be noticed in the compression and expansion strokes clearly. The piston velocity will start to increase near the BDC and decrease near the TDC in the case of the sinusoidal load. However, the two profiles share similar behavior during the combustion process; this may indicate that the load shape has a little effect on the ignition process. 


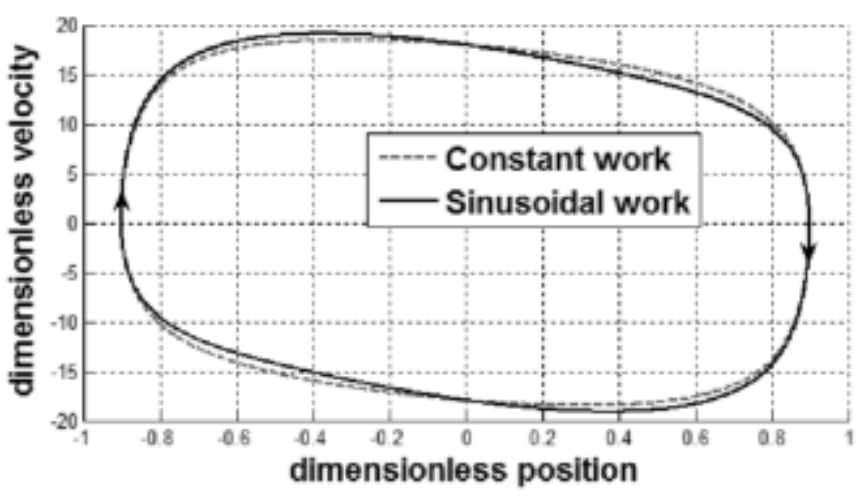

Figure 12 Effect of load profile of the piston dynamics [41].

\subsection{Thermodynamic sub-model}

The second primary sub-model is the in-cylinder thermodynamics. This sub-model includes the HRR calculations and all of the fuel chemistry. Many approaches can be used to estimate the heat released by the combustion. The empirical approach, which is represented by correlations like the Wiebe function, is commonly used, but its accuracy depends on the correlated parameters and cannot be applied generically [6]. Chemical kinetics considered one of the best tools because of its ability in predicting the ignition timing and heat release as well as the emissions products of the combustion event [7]. The kinetics sub-model includes multiple species and pathways, rather than the combination of a fraction of fuel burned with a complete combustion enthalpy. In addition to the heat release, the thermodynamics sub-model includes calculations to predict cylinder heat loss and gas exchange.

In this research, the thermodynamic sub-model is the central of the all investigations because it includes all the chemical kinetic calculations needed to represent the interactions of the air-fuel mixture. The sub-model covers the main engine processes including the intake charge compression, combustion, expansion and scavenging.

The first law of thermodynamics, in addition to the ideal gas law, provides the foundation for the thermodynamic model. These two laws are given in equations (4.7) and (4.8), respectively.

$\frac{d U}{d t}=-P \frac{d V}{d t}-\dot{Q}_{H T}+\dot{Q}_{c o m b}$

$V \frac{d P}{d t}+P \frac{d V}{d t}=m R \frac{d T}{d t}$

In equation (3.14), the time rate of change of the internal energy of the cylinder mixture $(d U / d t)$ is dependent on cylinder pressure $(P)$, the time derivative of cylinder volume $(d V / d t)$, and rate of heat exchanged by heat transfer $\left(\dot{Q}_{H T}\right)$ and fuel combustion $\left(\dot{Q}_{c o m b}\right)$. In order to obtain the piston position, equations (3.8), (3.13), and (3.14) need to be solved as a system using ODE solver.

The cylinder charge is represented as a perfectly mixed, ideal gas; compression and expansion are calculated with temperature dependent specific heat ratio and heat transfer between the gas and cylinder walls; combustion heat release is predicted with a chemical kinetics full reaction mechanism; and the gas exchange process is simplified to be perfect and instantaneous. The spring force is linear with respect to displacement and no material restrictions. 


\subsubsection{Chemical kinetics model}

The chemical kinetics is responsible about the in-cylinder species reactions within a PSR reactor where the reactants are perfectly mixed and burn homogenously. This kind of reactor is useful for examining highly mixed IC engines, residence time issues and high temperature chemical kinetics [121]. Cantera solves the time-dependent reaction equations where those equations represent the evolution of the chemical and thermodynamic state of the reactor [117]. The heat rate of combustion is calculated using the following formula [110]:

$\dot{Q}_{c o m b}=-V \cdot \sum_{i=1}^{N}\left(\dot{\omega}_{i} \cdot h_{i, m}\right)$

Where $\dot{\omega}_{i}$ is the net production rate of the species $i, h_{i, m}$ is the molar enthalpy of the species $i$, and $\mathrm{N}$ is the total number of species involved in the reaction mechanism.

The contents of PSR reactor follow species reactions according to a specified kinetic mechanism. A variety of reaction mechanisms is available and used throughout the literature. The reaction mechanism used in this study is the well-known GRI3.0 [113] mechanism. This mechanism includes 53 species with more than 300 reactions of these species. Many programs are available to solve the reaction equations such as Cantera ${ }^{\circ}$, which is used in this study.

To give a better idea about the nature of the reactions which are involved inside the mechanism, a global reaction demonstration is helpful. For example, in the case of GRI.30 mechanism, hydrocarbon fuels in the form of $\mathrm{C}_{x} \mathrm{H}_{y}$ can be represented in two ways. The first (equation 10) is a single global reaction [122].

$\mathrm{C}_{x} \mathrm{H}_{y}+(x+y / 4) \mathrm{O}_{2} \stackrel{K_{G}}{\rightarrow} x \mathrm{CO}_{2}+(y / 2) \mathrm{H}_{2} \mathrm{O}$

Alternatively, a multi-step reaction process like that given in equations (3.17) to (3.20) can represent the total reaction.

$$
\begin{aligned}
& \mathrm{C}_{n} \mathrm{H}_{2 n+2} \rightarrow(n / 2) \mathrm{C}_{2} \mathrm{H}_{4}+\mathrm{H}_{2} \\
& \mathrm{C}_{2} \mathrm{H}_{4}+\mathrm{O}_{2} \rightarrow 2 \mathrm{CO}+2 \mathrm{H}_{2} \\
& \mathrm{CO}+(1 / 2) \mathrm{O}_{2} \rightarrow \mathrm{CO}_{2} \\
& \mathrm{H}_{2}+(1 / 2) \mathrm{O}_{2} \rightarrow \mathrm{H}_{2} \mathrm{O}
\end{aligned}
$$

The above equations represent the basic destructive and constructive reactions process for the fuel. Intermediate hydrocarbons like $\left(\mathrm{C}_{2} \mathrm{H}_{4}\right)$ will be produced at the first stage of combustion where a bonds breaking is happen to the fuel molecules. These new less branches hydrocarbons will be oxidized. The products of the oxidation will be oxidized also producing the final combustion products $\left(\mathrm{CO}_{2}\right.$ and $\left.\mathrm{H}_{2} \mathrm{O}\right)$. For the full reaction mechanism, both destructive and constructive reactions occur. Destructive reactions include those that occur as the fuel is broken down. Constructive reactions are those that result in the formations of many of intermediate products like $\mathrm{CO}_{2}$ and $\mathrm{H}_{2} \mathrm{O}$. Additionally, these reactions continue both backward and forward simultaneously until the final chemical equilibrium state is reached [113].

\subsubsection{Heat transfer model}

A modified version of Woschni's correlation has been used to describe the heat transfer between the cylinder gas and wall of the engine. The correlation considers the nature of the free piston dynamics in addition to that of the $\mathrm{HCCl}$ combustion. The heat transfer coefficient and the heat transfer rate are described in equations (3.21) and (3.22), respectively [52, 123]

$h=3.26 \cdot B^{-0.2} P^{0.8} T^{-0.55} V_{P}^{0.8}$ 
Where $\mathrm{B}$ is the used engine bore, $\mathrm{p}$ and $\mathrm{T}$ are the instantaneous in-cylinder pressure and temperature and $V$ is the mean velocity of the mixture gases and it is assumed equal to the mean velocity of the piston, therefore the heat loss rate will be [52]:

$\dot{\mathrm{Q}}_{\text {loss }}=h \cdot A_{\text {wall }} \cdot\left(T-T_{\text {wall }}\right)$

The heat transfer coefficient $(h)$ is dependent on the cylinder pressure $(P)$ and temperature $(T)$, and cylinder gas velocity term $V_{P}$ which is empirically correlated [123]. The heat transfer then depends on the instantaneous surface area of the cylinder $\left(A_{\text {wall }}\right)$ and wall temperature $\left(T_{\text {wall }}\right)$. For a simulation, the wall temperature is calculated as the mean wall temperature of its corresponding adiabatic cycle.

\subsection{Simulation procedure}

An iterative procedure between the two sub-models (using MATLA ${ }^{\circ}$ and Cantera toolboxes) is used to simulate engine performance. The simulation starts with the dynamic model by applying the force balance on the translator. The translator initial position is set to coincide with the port closing position and its initial position is set to zero. Motion of the translator results from the acceleration computed from the force balance. Integration of acceleration yields the position profile, which is then used in the thermodynamic model to calculate the cylinder pressure based on intake conditions and heat loss. The point of ignition of the fuel is calculated using the chemical kinetics model and depends on cylinder mixture conditions. For a given simulation, the intake conditions, wall temperature, and heat transfer correlation remain fixed so that the cylinder charge is the same at each cycle. The point of ignition varies from cycle to cycle due to cyclic variance in the translator velocity at the start of compression stroke.

The simulation process is explained in Figure 13 where all the previous models are described within the process flowchart. The procedure starts with the dynamic model, which calculates initial position data for the translator based on initial conditions assumed within the engine cylinder. These position data are then supplied to the thermodynamic and chemical kinetics models represented in MATLAB ${ }^{\circ}$ and Cantera ${ }^{\circ}$. Then, the thermodynamic data (specifically the cylinder pressure) are returned to the dynamic model where the position and velocity of the translator are recalculated. This iterative procedure is terminated once the mean squared error between subsequently calculated volume profiles is below a specified tolerance $\left(10^{-8}\right)$, and the total procedure is repeated for each engine cycle.

To simplify the simulation in this study, the scavenging process is assumed to be perfect, meaning no cylinder gas is retained from cycle to cycle, and all of the conditions in the cylinder are returned to the intake conditions immediately when the ports open [54]. 


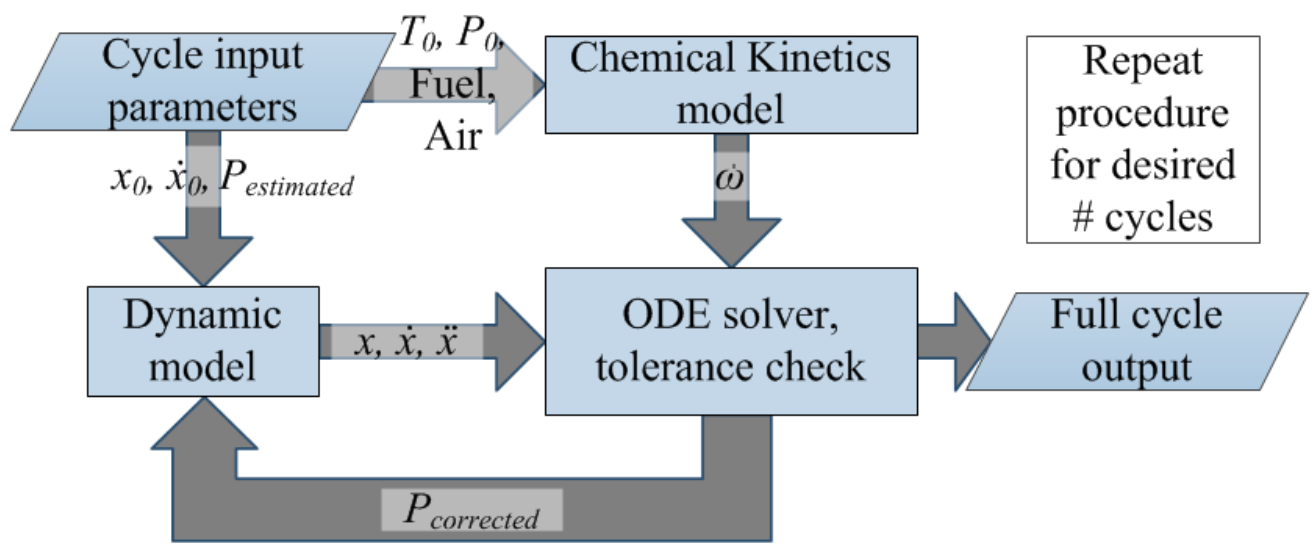

Figure 13 Flow chart diagram of the simulation model.

\subsection{Simulation pre-processing}

To start the simulation process, some pre-processing steps are needed. Defining the fuel composition and the amount of needed stoichiometric air are included under this task. To speed up the calculations, a $M A T L A B^{\circledR}$ function was written to evaluate the stoichiometric air quantities need by the implemented fuel.

The simulation will start under the influence of assumed motor pressure. A polytropic process is considered at first to create the initialization profile. A variable specific heat ratio is assumed within this scheme to include the effect of increasing charge temperature. In the main code, the produced piston volume values and their rate of change generate the estimated pressure and temperature profiles. At this stage, the combustion model by Cantera solves the reactions equations for the actual pressure values. At the same time, the model reflects the effect of the combustion process on the pressure diagram in the next iteration. Finally, the new profiles are used to create a corrected volume profile at the dynamic model. This process will keep in back and forth until an enough tolerance point is reached.

\subsection{Multi-cycle simulation}

The simulation is described by 100 cycles of engine operation under the stoichiometric conditions. The average frequency of those cycles is found to be $125 \mathrm{~Hz}$. $\mathrm{HCCl}$ combustion occurs at each cycle if the fuel-air mixture is compressed to the point of auto-ignition. All of the main processes for each cycle are demonstrated in Figure 14, the figure represents the in-cylinder pressure variation with respect to the piston position. In the figure, multiple profiles are evident, showing the initialization cycle with the earliest combustion and shortest stroke length. After the initialization cycle, the model controller adjusts the alternator load to achieve near steady state conditions.

Around top dead center (TDC), the adverse work region is indicated. Zooming in on this region would reveal an inversion of the pressure-position profile. Negative work occurs on the piston as it moves through top dead center because of two phenomena. First, ignition of the rapid $\mathrm{HCCl}$ combustion event occurs sufficiently prior to top dead center. Second, heat transfer detracts from the combustion energy held within the cylinder more rapidly than the cylinder pressure can return the translator through its expansion stroke. 


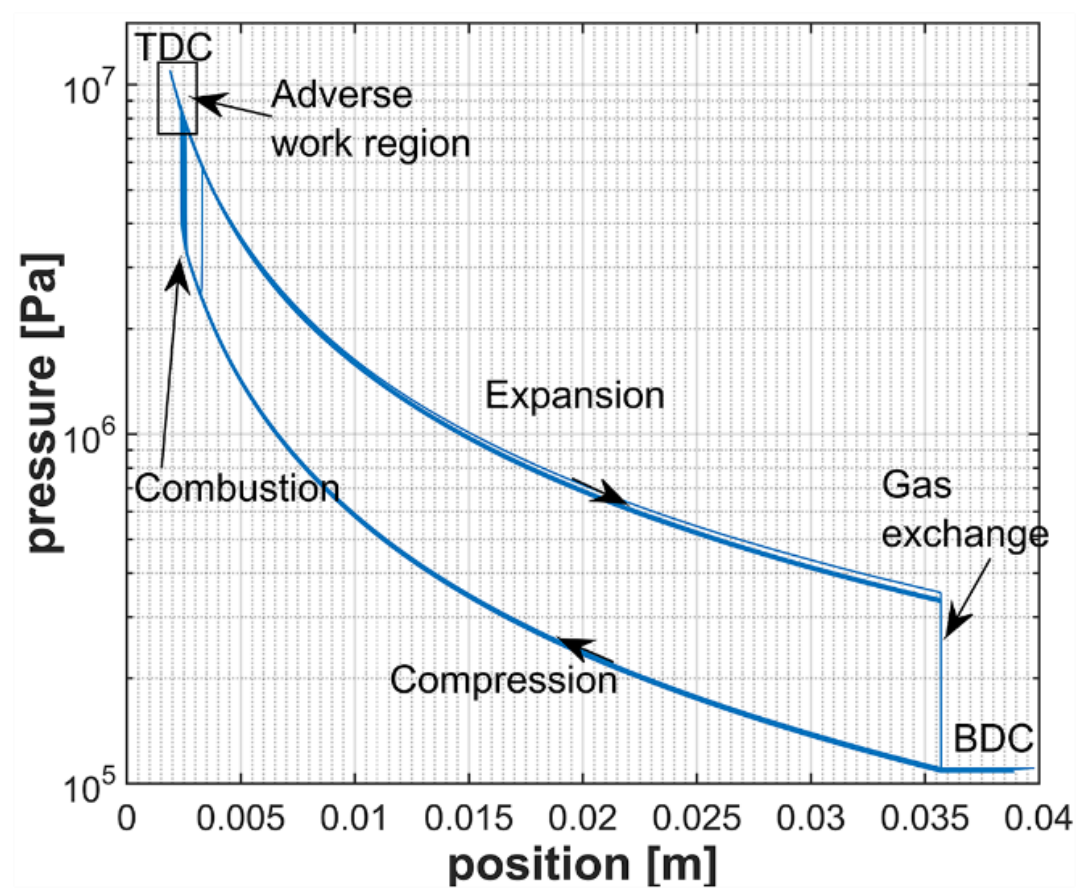

Figure 14 Pressure versus position diagram.

Figure 15 illustrates the solution of the dynamic model stemming from the equation of the forces balance where the translator position, velocity, and acceleration are calculated for the current simulation. The number of simulated cycles presented in the figure obscures the shape of the path taken by the piston during any single cycle, but Figure 16 helps to provide a sense of scale for the piston motion. Referring back to Figure 14, the minimum volume is achieved when the translator is at its minimum position (TDC). Figure 15 shows that the translator oscillates so that the piston is always between 0 and $40 \mathrm{~mm}$ away from the cylinder head. This follows because the cylinder pressure is infinite at $0 \mathrm{~mm}$. Also, the given average frequency of the simulated engine $(125 \mathrm{~Hz})$ means that the 100 simulated cycles are completed in less than 1 second. 

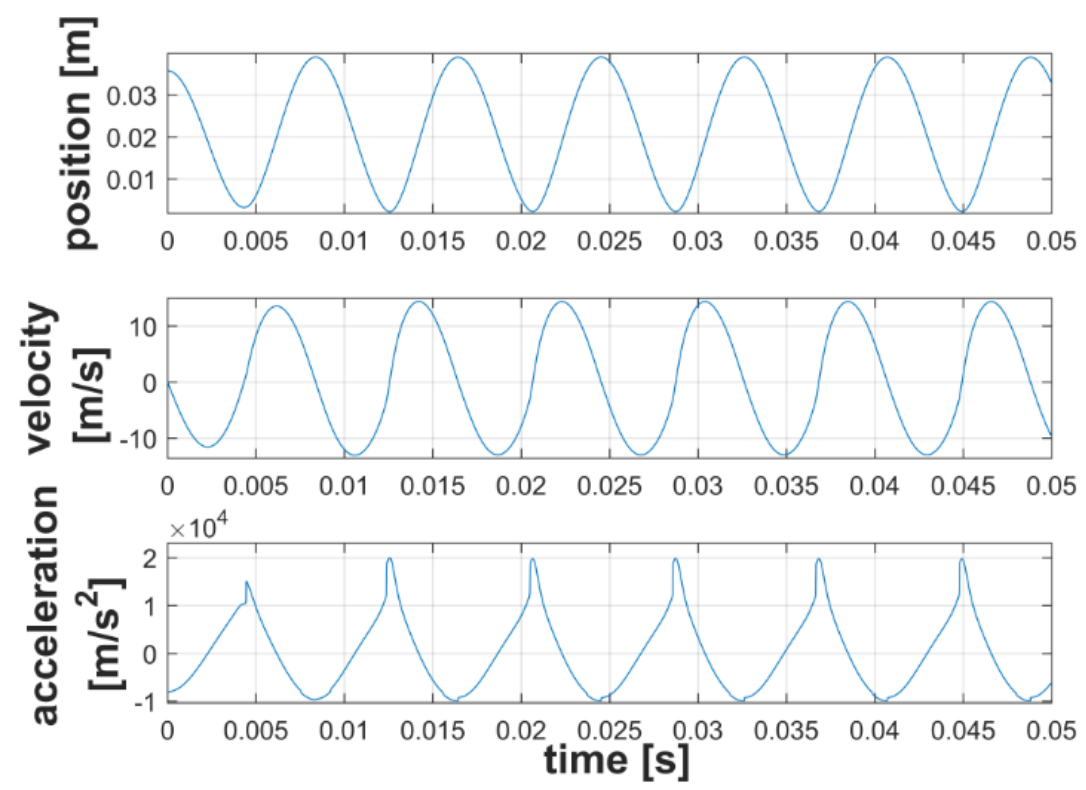

Figure 15 Position, velocity, and acceleration versus time for the first 0.2 seconds of simulated operation.

Figure 16 describes the translator velocity profile based on its position. The profile is 'egg-shaped' with the TDC and BDC positions along its major axis (the horizontal axis corresponding to translator velocity equal to zero). The profile lacks symmetry because the maximum velocity achieved by the translator differs between compression and expansion strokes. The first half of the cycle (the compression stroke) is dominated by the compression spring, while the second has added influence from the cylinder pressure. The simulation is initialized at bottom dead center at the position where the ports close and cylinder compression begins. The force of the spring accelerates the translator towards the cylinder head. Near top dead center, the fuel-air mixture reaches the auto-ignition point. At this point, based on the chemical kinetics model, the cylinder mixture burns rapidly causing a sharp rise in cylinder pressure. The high force from the cylinder pressure slows and stops the translator, then returns it through the expansion stroke.

Figure 17 continues to illustrate the dynamic behavior of the translator through the acceleration versus velocity plot. As in Figure 17, the initial state of the translator is indicated. The acceleration plot provides further insight into the effects of cylinder pressure on translator dynamics. The combustion event follows cylinder compression and is evident as a steep rise in acceleration due to the heat release and associated pressure rise in the cylinder. Following the combustion and expansions processes two instantaneous changes are seen in the accleration profile around bottom dead center. The first along the path occurs when engine ports open allowing for instantaneous gas exchange (per the given assumption). The second is an artifact of the controller which changes the alternator load constant instantaneously at the bottom dead center position in order to encourage system stability. 


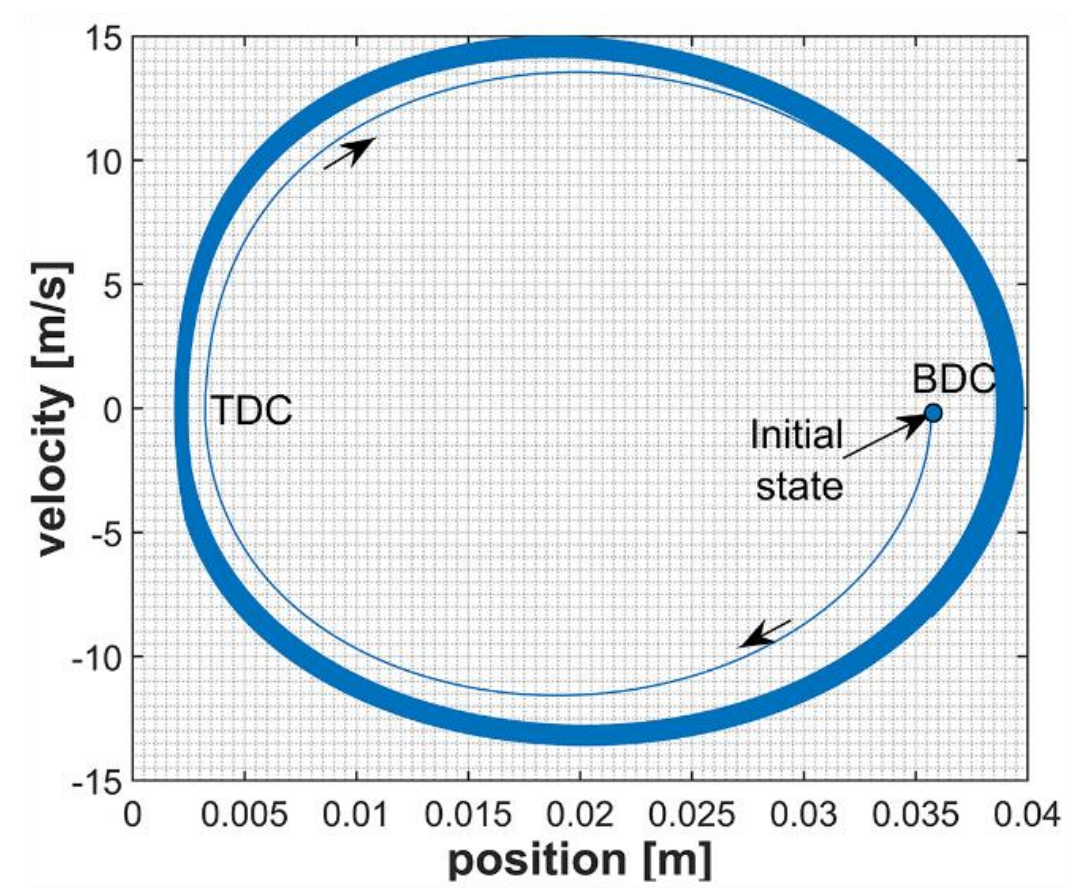

Figure 16 Velocity versus position plot for 100 simulated cycles.

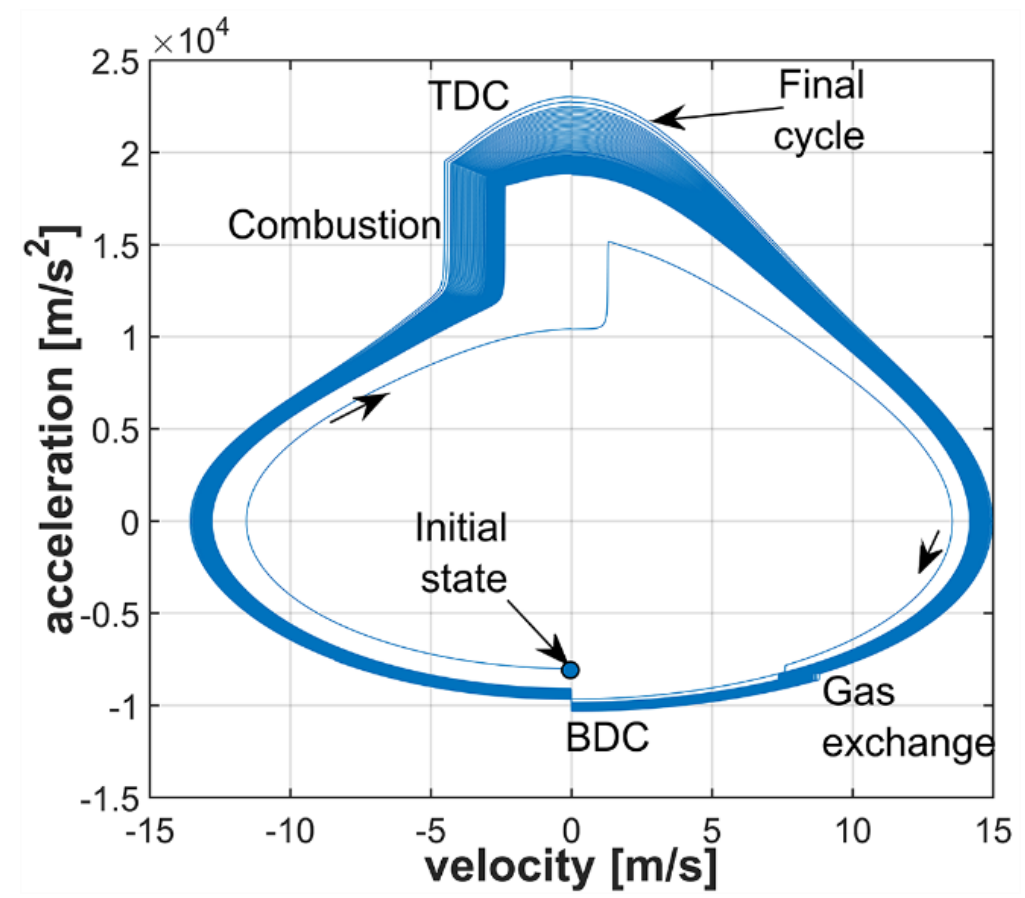

Figure 17 Acceleration versus velocity plot for 100 simulated cycles.

Figure 18 shows the cylinder pressure and temperature with respsect to time for the first four cycles of simulated operation. In the simulation, fuel and air are charged to the engine cylinder at atmospheric pressure and high temperature (relative to atmospheric). The high temperature helps to ensure that combustion is achieved within the engine. The inlet conditions applied in the simulation result in cylinder peak pressure of 100 bar and peak temperature of $2830^{\circ} \mathrm{C}$, while the average CR is 18 . In Figure 18 , the 
variation between the first two cycles is shown to be significantly greater than the variaiton between subsequent cycles. This is due to the initial conditions used in the first cycle. After the first cycle, ignition delay is found to be around 0.005 seconds while the combustion duration is found to be about 0.001 milliseconds. Generally, these values will vary based on the type of fuel, inlet conditions, and engine parameters. It should be mentrioned that the igntion delay is calculated form the start of the up to the start of of igntion process. The start of the igntion is found by tracking the slop of the pressure diagram $\left(\frac{d p}{d t}\right)$ where the time that this slop change sudenly is consider as the igntion time.

During the simulation, the percentage of the overall combustion heat energy converted to output work by the alternator is $47 \%$. The friction model used within the simulation leads to only $0.4 \%$ of the combustion energy dissipation. Friction in a real linear engine could be higher than that found in the current study. Frictional losses reported by DLR researchers amounted to 6\% [124]. Thus, it is expected that the model used within the current simulation underpredicted the frictional losses. Correction of this model will reduce the overall efficiency for sure.
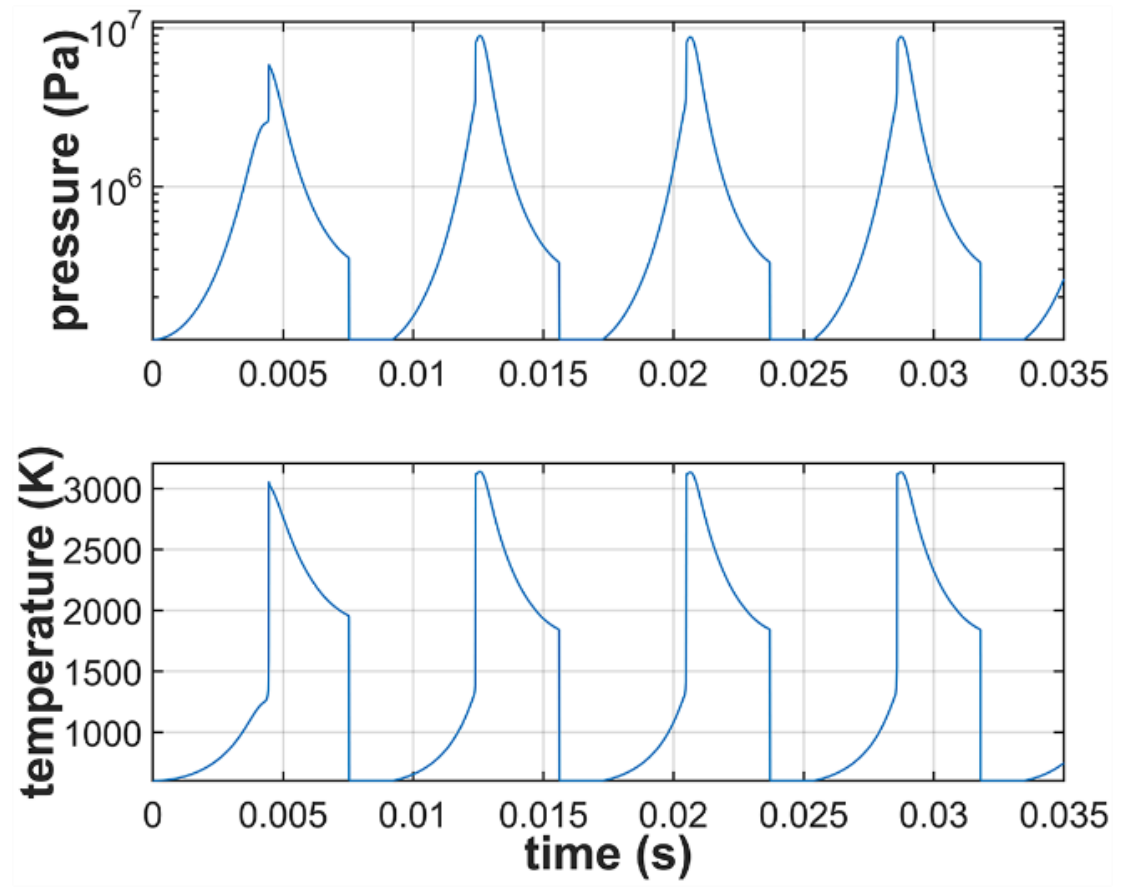

Figure 18 The first four cycles of pressure and temperature versus time.

\subsection{Model comparison and validation}

The new concept of using Cantera as a chemical kinetics tool in internal combustion engines simulations requires the validation of the whole system. This validation will cover the chemical kinetics equations, their solver and any other related sub-models. Unfortunately, the models that represent freepiston engines using chemical kinetics approach are very rare in the literature. Based on this, the validation should be comprehensive and not limited for free-piston devices. For this purpose, a conventional engine model was built and compared to Chemkin ${ }^{\circledR}$ model with same features. The engine geometries and parameters were used as in a Chemkin ${ }^{\circledR} \mathrm{HCCl}$ combustion engine example. All of the 
parameters are illustrated in Table 2 and the required equations that represent the dynamic part of the model are shown below as well.

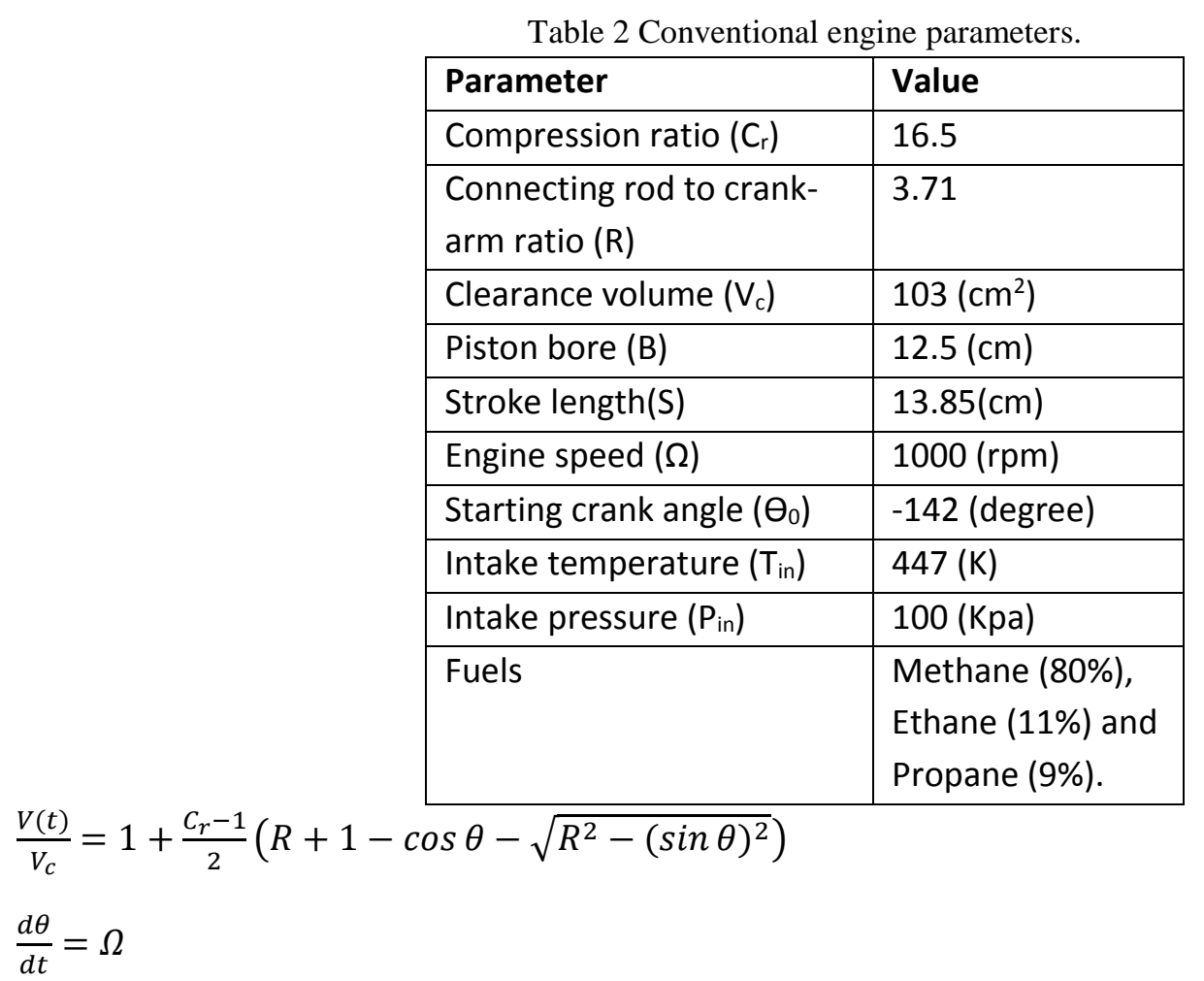

The rest of the model equations that belong to the thermodynamic sub-model are the same as in the section 3.4. To compare the Cantera model to the Chemkin ${ }^{\circledR}$ one precisely, the conditions and the parameters were set the same. GRI3.0 mechanism was used to present the species reactions in the both cases. Thus, the differences between the two models will be due to the calculations tools difference only. One engine cycle was performed to compare the Cantera model to the Chemkin ${ }^{\circledR}$ example and the results are shown in Figure 19, Figure 20 and Figure 21 receptively. The pressure and temperature diagrams are zoomed near the peak to show the matching between the two models. The results indicate that the two models are extremely similar in all cycle processes. The combustion event, which is the main stage in the cycle, is identical in the Cantera model. Although the model does not belong to a specific engine, but Cantera tools showed an excellent capturing of different engine cycle processes. 


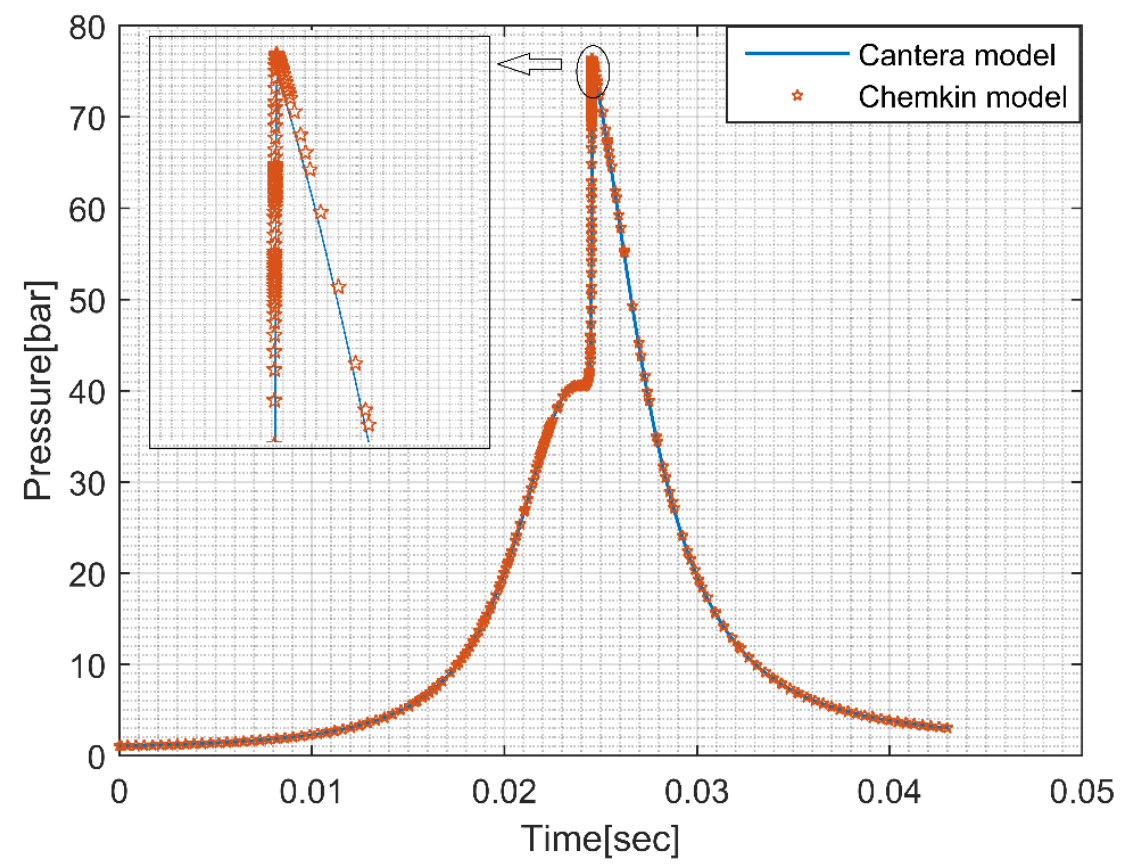

Figure 19 In-cylinder pressure for Cantera and Chemkin® models.

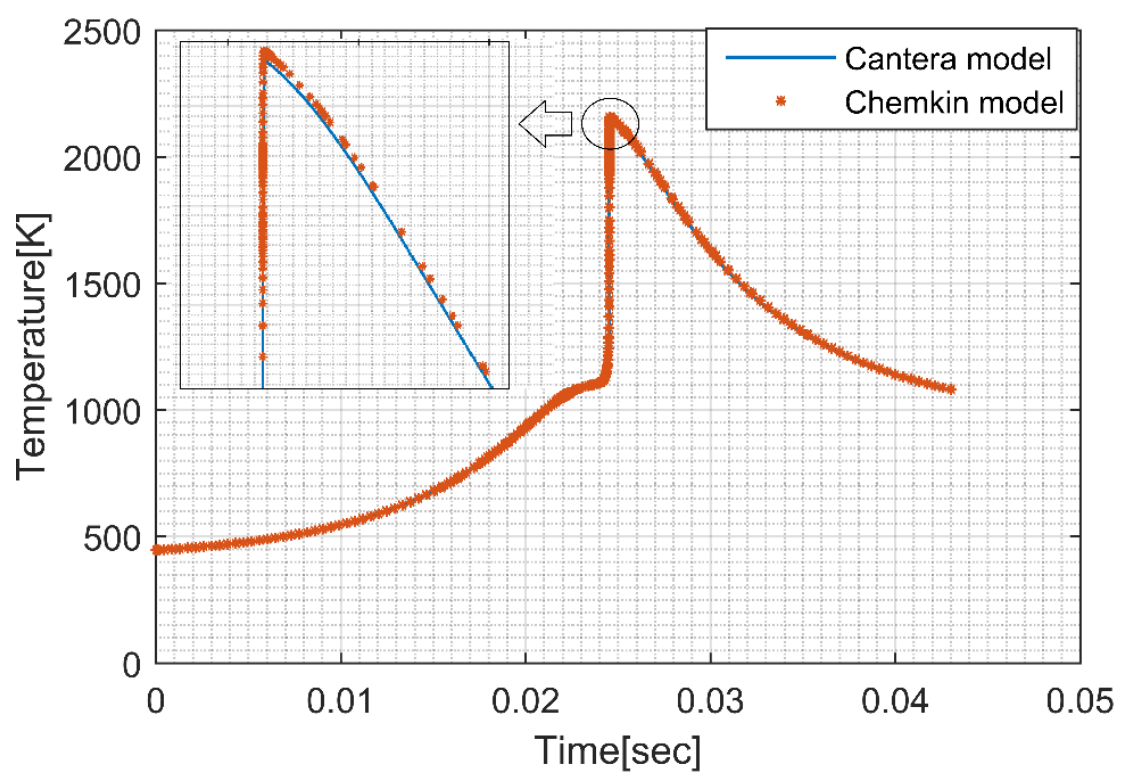

Figure 20 In-cylinder temperature for Cantera and Chemkin® models.

It should be mentioned that the scavenging process in the two models is not included since no information in the Chemkin ${ }^{\circledR}$ example is found as it can be seen in Figure 21. In general, scavenging is kind of finishing and beginning stage process, which has less effect on the combustion process than the other stages. In addition, it is expected that including any scavenging technique in the two models will not affect the overall matching, as they are identical. 


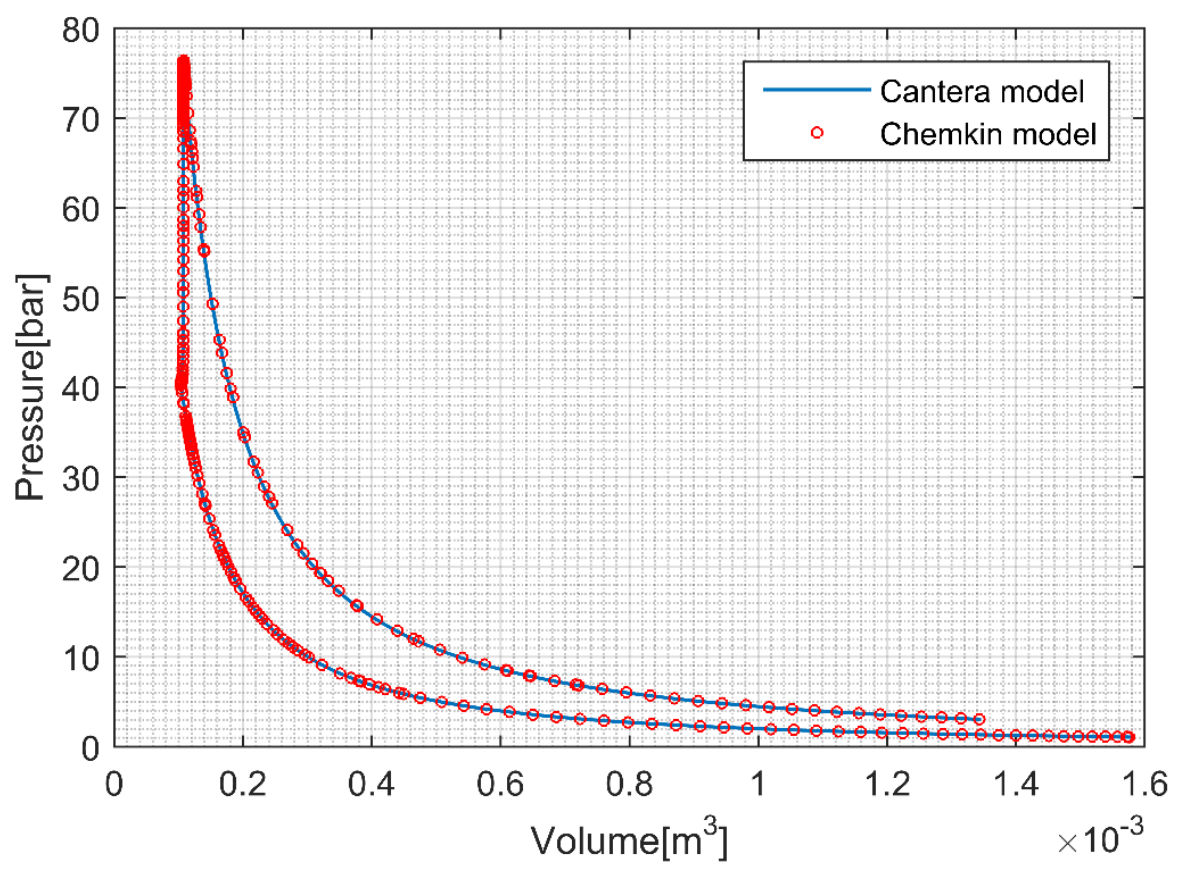

Figure 21 Pressure-volume diagram for Cantera and Chemkin models.

The free-piston engine model was validated as well in this study where the alternator PD controller is capable of driving the device to near steady state conditions. The model validation can be performed to compare the simulation results with either experimental data or other numerical model results found in the literature. A free piston engine model produced by Goldsborough and Van Blarigan from SNL [18] is presented for comparison to the current numerical model. The engine geometry and parameters from the SNL model have been used within the Cantera/MATLAB ${ }^{\circ}$ of this dissertation to simulate the engine operation with $\mathrm{HCCl}$ combustion of $\mathrm{H}_{2}$ fuel. The models parameters are described in Table 3.

Figure 22 shows the pressure-time traces for both the current model - the Cantera/MATLAB ${ }^{\circ}$ model - and the SNL numeric model. The comparisons show good correlation between the two models with the greatest disparity arising during the combustion process. This is likely due to a difference in ignition timing between the two models and could be caused by a variety of reasons including the reaction mechanism and the temperature distribution along the cylinder walls. For the autoignition combustion process, the number of active reactions is the dominant factor in determining the ignition timing. Reduced mechanism, the fast species reactions are neglected meaning that the model describes only the major reactions [111]. Despite the difference in ignition timing, the two cycles are similar in peak pressure, indicating that peak pressure is not only dependent on ignition timing. Factors such as air to fuel ratio and compression ratio can also affect peak pressure [6]. 


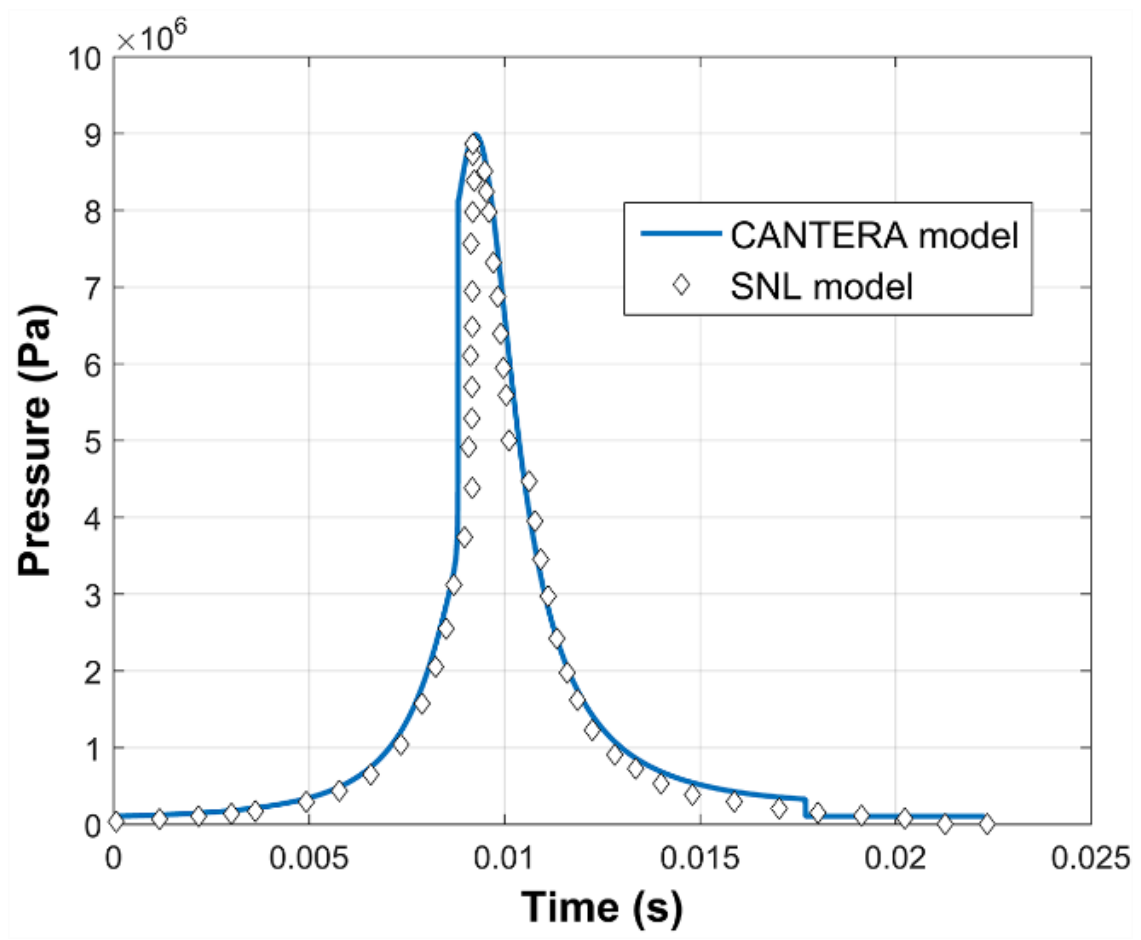

Figure 22 Single cycle comparison between current model with Cantera and the SNL model.

Table 3 Simulated SNL engine models specifications.

\begin{tabular}{cc}
\hline Parameter & Value \\
\hline Cylinder bore $(\mathbf{B})$ & $7.00 \mathrm{~cm}$ \\
\hline Piston mass $(\mathbf{m})$ & $2700 \mathrm{~g}$ \\
\hline Typical stroke $\left(\mathbf{S}_{\mathrm{max}}\right)$ & $16.40 \mathrm{~cm}$ \\
\hline Exhaust port top & $14.00 \mathrm{~cm}$ \\
\hline Intake port top & $14.15 \mathrm{~cm}$ \\
\hline Exhaust port bottom & $17.25 \mathrm{~cm}$ \\
\hline Intake port bottom & $17.25 \mathrm{~cm}$ \\
\hline Intake temperature & $300 \mathrm{~K}$ \\
( $\left.\mathbf{T}_{\text {in }}\right)$ & \\
\hline Intake pressure $\left(\mathbf{P}_{\text {in }}\right)$ & $1.50 \mathrm{bar}$ \\
\hline Wall temperature $\left(\mathbf{T}_{\mathrm{w}}\right)$ & $700 \mathrm{~K}$ \\
\hline
\end{tabular}




\section{Chapter 4. Model control}

Controlling of the free piston engine is of high significance for steady and efficient operation, especially with $\mathrm{HCCl}$ combustion. Cycle to cycle variations of stroke length, top dead center location, and combustion process leads to high difficulty in achieving smooth and steady performance over many cycles [31]. In this study, the implemented model controllers are of a first generation kind. Many controllers have been applied to investigate the most stable and efficient controller in achieving the required number of cycles.

\subsection{Proportional-derivative controller}

The PD (proportional-derivative) controller used in the investigation is illustrated in Figure 23. For each cycle, when the translator is at bottom dead center, the error between the previous and current bottom dead center cycle locations is calculated. The proportional component is added to the derivative component and combined with the initial alternator load constant. The derivative component serves to compensate for the rate of change of the bottom dead center location. The effect of the derivative component affects the output by decreasing it as the process variable is increasing unsteadily. Such effect is known also as a damping effect since the oscillations in the solution will be minimized gradually. Equation 4.1 explains the mathematical form of the used PD controller. In this equation, the alternator load constant is $C_{A}, B D C_{E R R O R}$ refers to the difference between the new and the old BDC values. Lastly, $K_{P}$ and $K_{D}$ are the proportional and derivative gain factors respectively. To initialize the simulation, the load constant is assumed to be 32 . For subsequent cycles, the load constant is calculated from the error with a proportional gain of 73 , and a derivative gain of 0.75 . The gain values are found through trial and error but are not optimized. Generally, ranges are available for the controller gain values that results in no cyclic variations over a number of cycles. Outside of these ranges, two phenomena are typically seen. If the controller allows excessively high alternator load, misfire occurs in the engine cylinder, eventually leading to engine stall. If the controller results in excessively low load, the engine is allowed to "ring-up" such that compression ratio and stroke continue to increase until unreasonable for realistic operation.

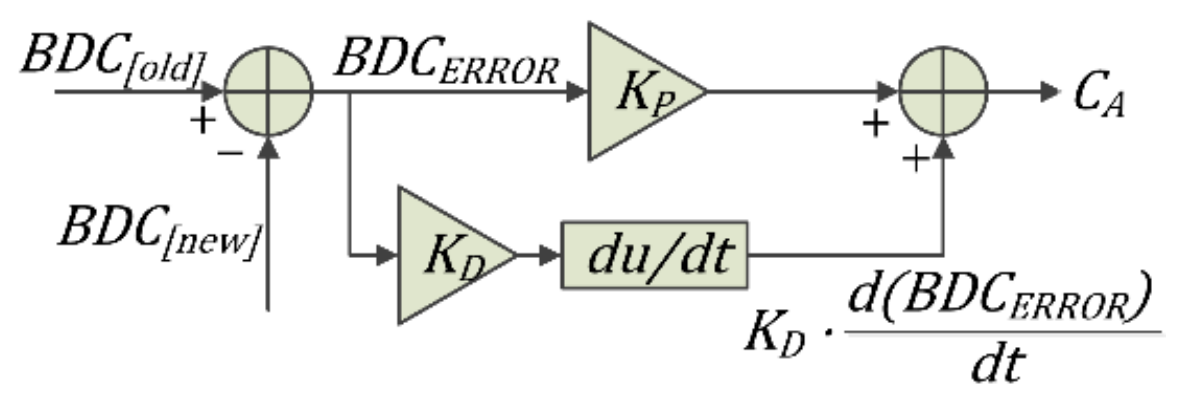

Figure 23 Proportional- derivative (PD) Controller diagram used in the simulation.

$$
\mathrm{C}_{\mathrm{A}}=\left(\mathrm{C}_{\mathrm{A}}\right)_{\text {old }} \times\left(\mathrm{K}_{\mathrm{P}} * \mathrm{BDC}_{\mathrm{ERROR}}+\mathrm{K}_{\mathrm{D}} * \frac{\mathrm{d}\left(\mathrm{BDC}_{\mathrm{ERROR}}\right)}{\mathrm{dt}}\right)
$$

The proportional and derivative components of the controller presented in this study are combined to encourage stability for the 100 cycles. Neither the gain values nor controller architecture are optimized, 
but two cases are presented for comparison. The two controllers are both of the same architecture shown in Figure 23 but with different gain values.

Figure 24 illustrates the cycle-to-cycle variations of the mean piston velocity for the two controllers being compared. Through the first 20 cycles, both controllers are capable of maintaining the engine translator to a mean velocity of approximately $7.8 \mathrm{~m} / \mathrm{s}$. However, the mean velocity traces corresponding to the two controllers diverge from each other. The comparison reveals that the controller with the lower gain values does not sufficiently raise the alternator load to compensate for the rising energy of the translator from cycle to cycle. In both cases, it seems that the mean velocity is likely to continue to rise. Technically, the investigation show that the piston speed has a significant effect on the engine performance, for example if this speed exceeds $8 \mathrm{~m} / \mathrm{s}$, a divergence in the controller trend will be observed as in Figure 24. This indicates the need to further study the controller design space to achieve true steady state conditions. At the same time, increasing the number of the operating cycles will end up with numerical oscillations due to the disability of the controller to deliver all of the system energy to the alternator.

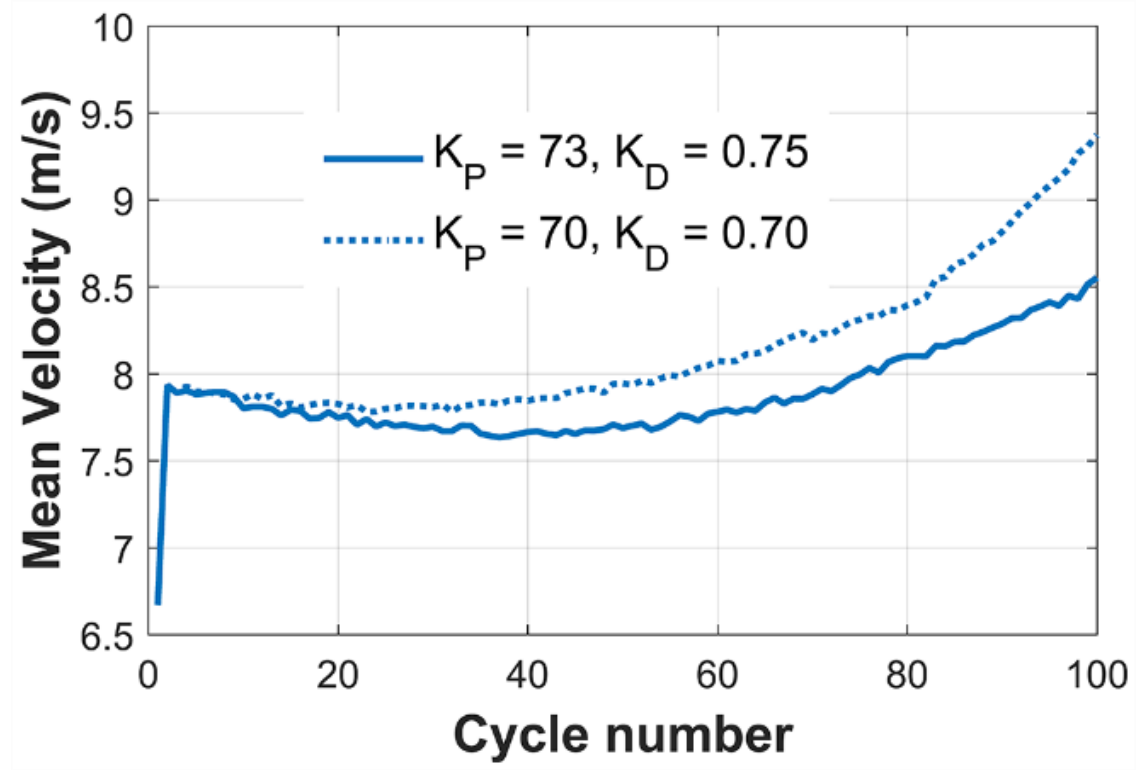

Figure 24 Cycle to cycle variations of piston mean velocity over 100 cycles for two controllers.

Next, Figure 25 compares the same two controllers with respect to their effect on compression ratio through the 100 cycles simulation. Compression ratio is an important factor for engine performance; on the one hand, low compression ratio is associated with poor efficiency and can result in poor or missed combustion events. On the other hand, high compression ratio can result in excessively high heat loss rates and overly advanced ignition timing, both detriment the thermal and combustion efficiency [56]. In addition, cylinder mixture blow-by past the piston rings increases with cylinder pressure, and mechanical failure can result from high compression ratio. In addition to the mean piston velocity, cycle to cycle variations of the free piston engine can be observed via changes of the compression ratio (related to changes of the stroke length). Compression ratio is allowed to vary because the piston of a free piston engine is not mechanically constrained within the system. Variation of compression ratio is related to cycle to cycle variations of cylinder temperature, pressure, gas velocity, mixture stratification, and start 
of combustion [125]. Thus, a strong interdependence between translator dynamics and compression ratio exists, and examination of the compression ratio over the length of the simulation offers many insights [126]. Figure 25 shows the variation of the compression ratio during the 100 cycles simulation under the influence of the two controllers. Similar to the mean velocity plots, the controllers cause an initial decrease in compression ratio followed by an increase. Again, the controller with the higher gain is able to better maintain the system stability, as evident by the smaller range of compression ratio achieved through the simulation. Although each cycle has the same intake condition, but the initial velocity of the piston is changing because of the increasing in the storage energy within the system. The accumulation in the system energy is happening gradually along the cycles, at the same time; the alternator will start losing its ability to absorb this energy, which results in increasing of the stroke length and therefore the compression ratio.

It is also evident that neither controller effectively drives the engine to true steady state operation. In general, the PD controller has the ability to run the engine model with a desired number of cycles. The complexity of the $\mathrm{HCCl}$ combustion mode and the nature of the free-piston engine restrict the controller from reaching any steady operation under the influence of the current controller.

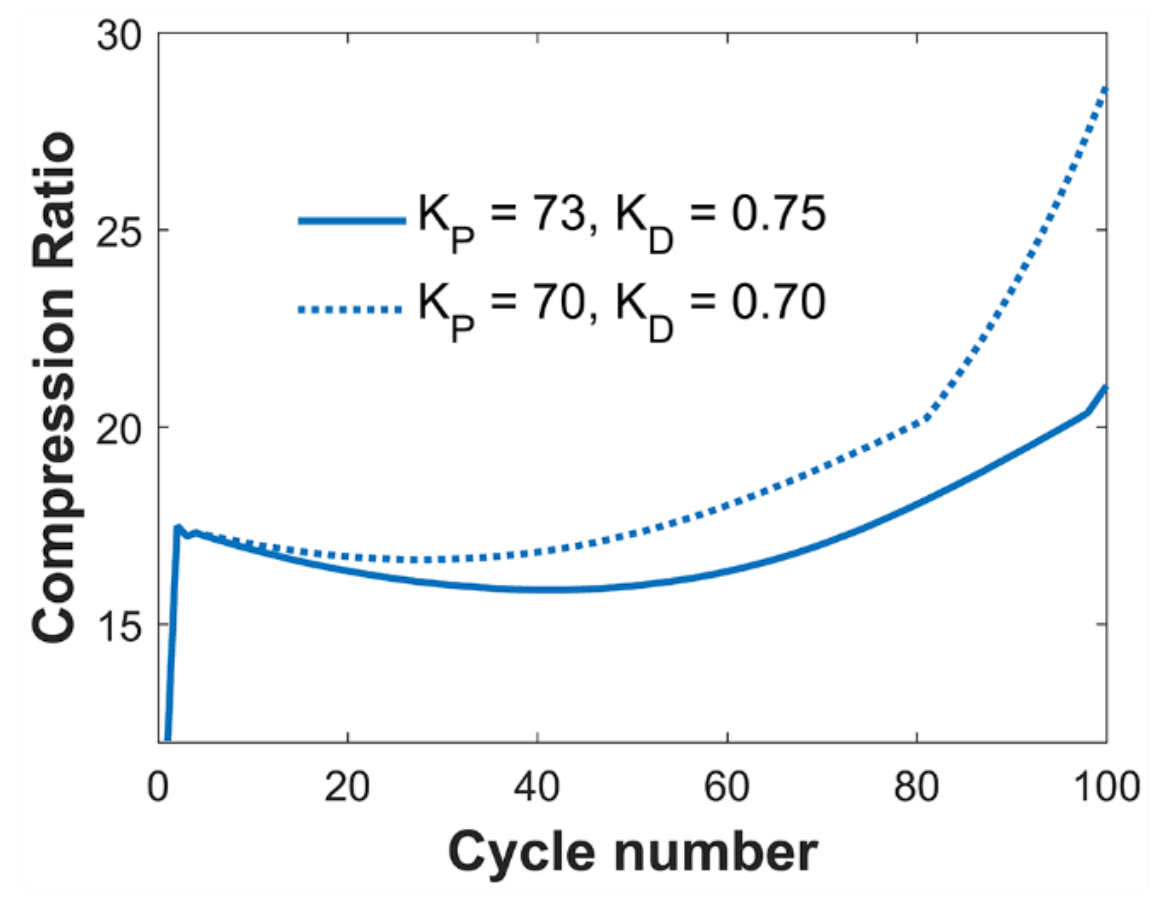

Figure 25 Cycle to cycle variations of cylinder compression ratio over 100 cycles for two controllers.

\subsection{Proportional-integral controller}

The second controller in this study is a proportional-integral controller. The controller was set to achieve a certain compression ratio ( $\left.\mathrm{CR}_{\text {Target }}\right)$ value within the simulation time. Figure 26 explains the main concept of the controller. To control the cycle compression ratio, the controller weight $\left(W_{a}\right)$ is allowed to change within an upper and lower values. The proportional part of controller corrects the controller 
weight based on the error between the actual and the desired compression ratios. In the other side, the integral part of the controller will integrate the error over the time and add it to the proportional result. The advantages of using the integral side of the controller focus on the fact that even small error will increase the integral component. Such response will be continually increased over time as long as the error is not zero, this behavior will drive the solution to the steady state (zero error point) [127] . The controller is mentioned in the mathematical form as in the following equations.

$\mathrm{F}_{\mathrm{W}}=\mathrm{W}_{\mathrm{A}} \cdot \mathrm{C}_{\mathrm{A}} \cdot \dot{\mathrm{x}}$

$\mathrm{W}_{\mathrm{A}}=1-\mathrm{K}_{\mathrm{P}} * \mathrm{ERROR}-\mathrm{K}_{\mathrm{I}} * \int \mathrm{ERROR} \cdot \mathrm{dt}$

ERROR $=\mathrm{CR}_{\text {Target }}-\mathrm{CR}_{\text {Actual }}$

In the previous equations, $F_{w}$ represents the alternator load, it gets a different form than the one used in the $\mathrm{PI}$ controller in order to include the effect of the load shape as well. $K_{\mathrm{p}}$ and $\mathrm{K}_{\mathrm{I}}$ are the proportional and integral gains respectively. $\mathrm{CR}_{\mathrm{Actual}}$ is the cycle actual compression ratio. $\dot{\mathrm{x}}$ is the piston instantaneous speed.

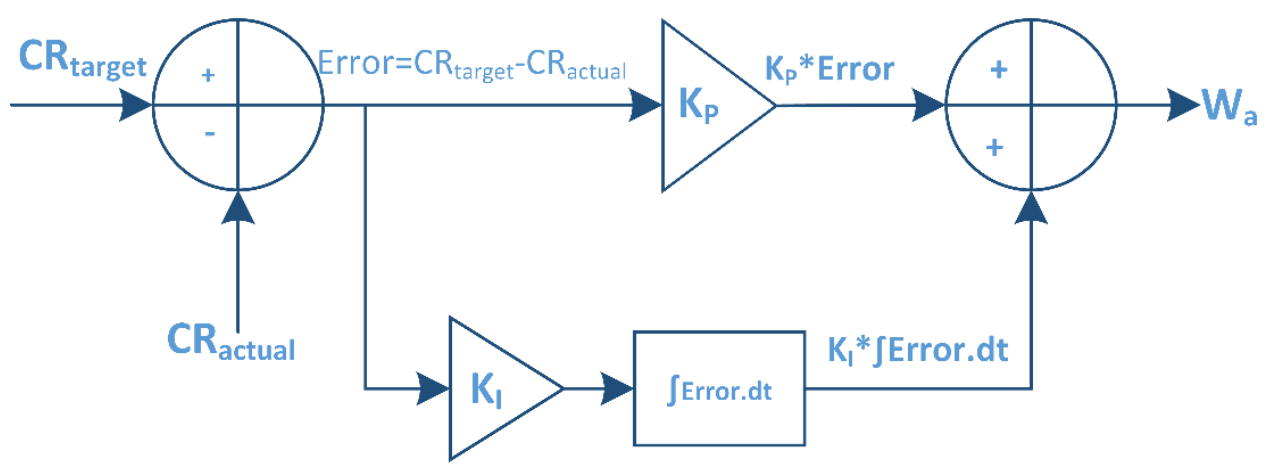

Figure 26 Propotional-integral controller diagram.

The results of implementing the PI controller are illustrated in Figure 27, the figure presents both of the compression ratio and the mean piston speed. Forty cycles of the engine operation were simulated under the influence of the PI controller. Figure 27 indicates a very interested observation after the first 10 cycles where a unique behavior is reached. The engine starts to fluctuate around the desired compression ratio value; the oscillation is uniform and steady along the simulation time, which indicate somehow a state of stability in the performance. Up to this level of the study, there is no specific or detailed explanation for this trend. However, extra tuning and calibration may come with the normal steady state behavior. The difficulty in such modifications is the time cost needed by the controller compared with the other controllers and the complex nature of the relation between the dynamics and the thermodynamics sides. The values of the controller gain, which used in the Figure 27 are 1.0 for both of the proportional and the integral components. The alternator coefficient $\left(C_{A}\right)$ is 4.5 , the desired compression ratio is 30 and finally, the upper and lower limit of the controller weight are 1.4 and 8 respectively.

Another aspect for the PI controller results is shown in Figure 28; the figure demonstrates the piston position profiles along the simulation time. It is obvious that the profile is changing between two main shapes only leaving a good chance for considering a steady state operation. Deciding if the controller is working under the steady state operation or not is difficult. The meshing process between the system 
dynamics and the thermodynamics is very critical point in the resulted behavior. The data exchanging between the two sub-models cannot be controlled by affecting one of the sub-models only. On the other hand, since the alternator load varies proportionally to the piston velocity, the trend of the compression ratio appears to have the ability of treating all the stored energy within the system. This conclusion is obvious in Figure 27 where the oscillations in the compression ratio start to be constant after only 15 cycles.

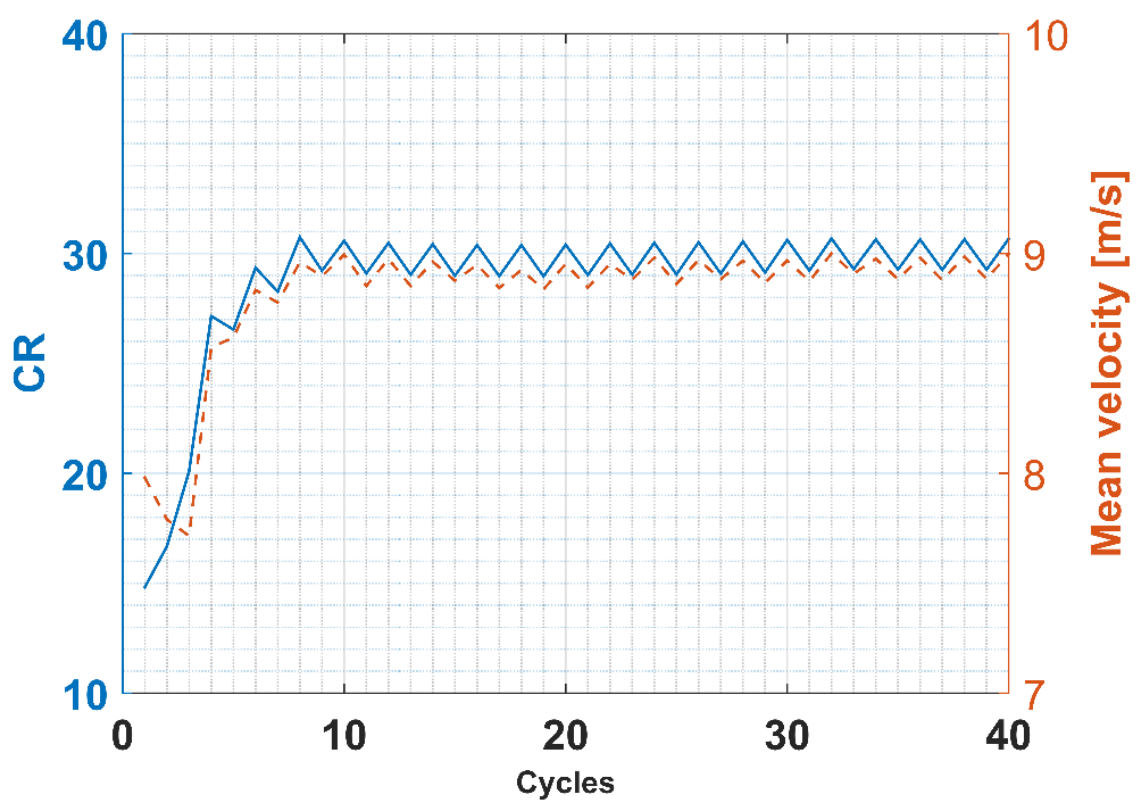

Figure 27 Compression ratio and mean piston variations.

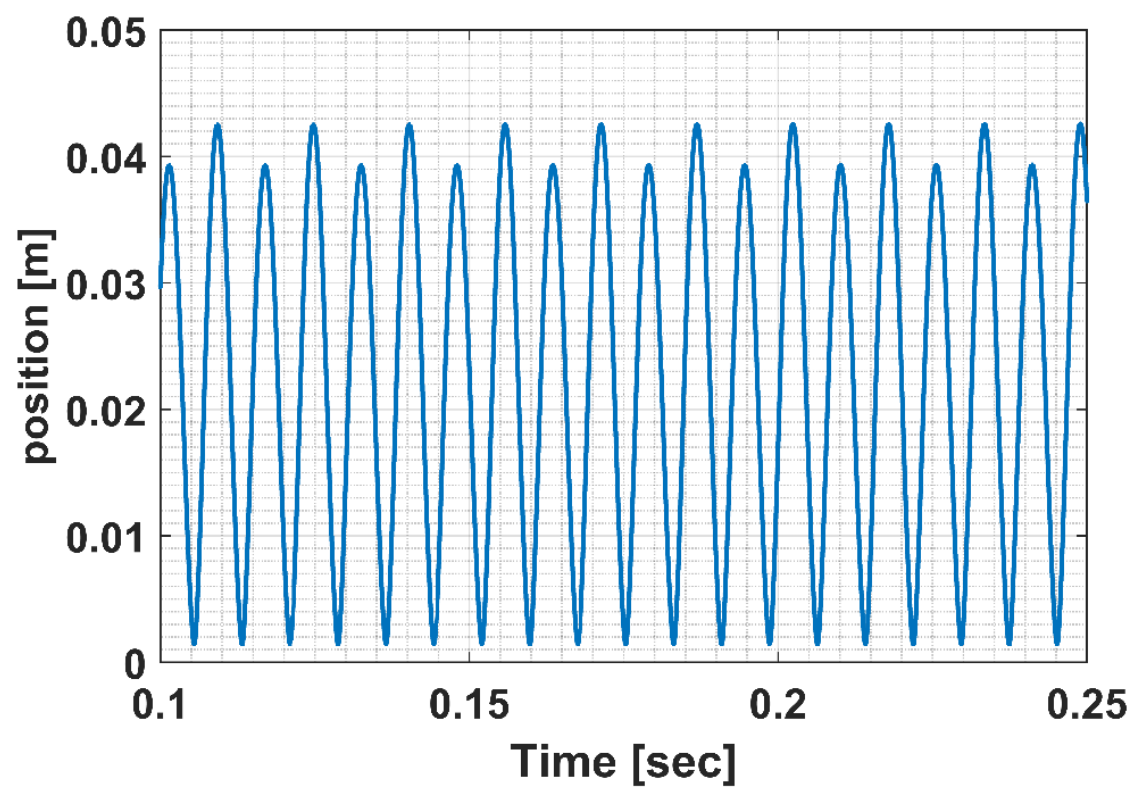

Figure 28 Piston position profile for the PI contoller. 


\subsection{Free-piston frequency}

In order to examine the frequency of the free-piston engine within the current model, parameters like the piston mass and spring stiffness have been examined. The figures below show the free-piston engine frequency at different piston mass and springs stiffness. Starting with the effect of the piston mass, Figure 29 describes the effect of three different piston masses on the engine frequency. The frequency appears to vary proportionally with the piston mass. For example, piston mass like $0.5 \mathrm{~kg}$ results with higher frequency $\left(125 \mathrm{H}_{z}\right)$ than a mass of $1.0 \mathrm{~kg}$ that produces less frequency $\left(95 \mathrm{H}_{\mathrm{z}}\right)$. The effect of the piston mass is slowing down the piston in both of the compression and expansion strokes. The high resulted piston inertia will restrict the piston movement especially near the BDC and TDC locations. In addition, the total computational time will increase for the same number of cycles. Theoretically, Figure 29 shows that there is no minimum frequency attached with the free piston engine operation, the motion profile will be the same regardless of the operating frequency [26].
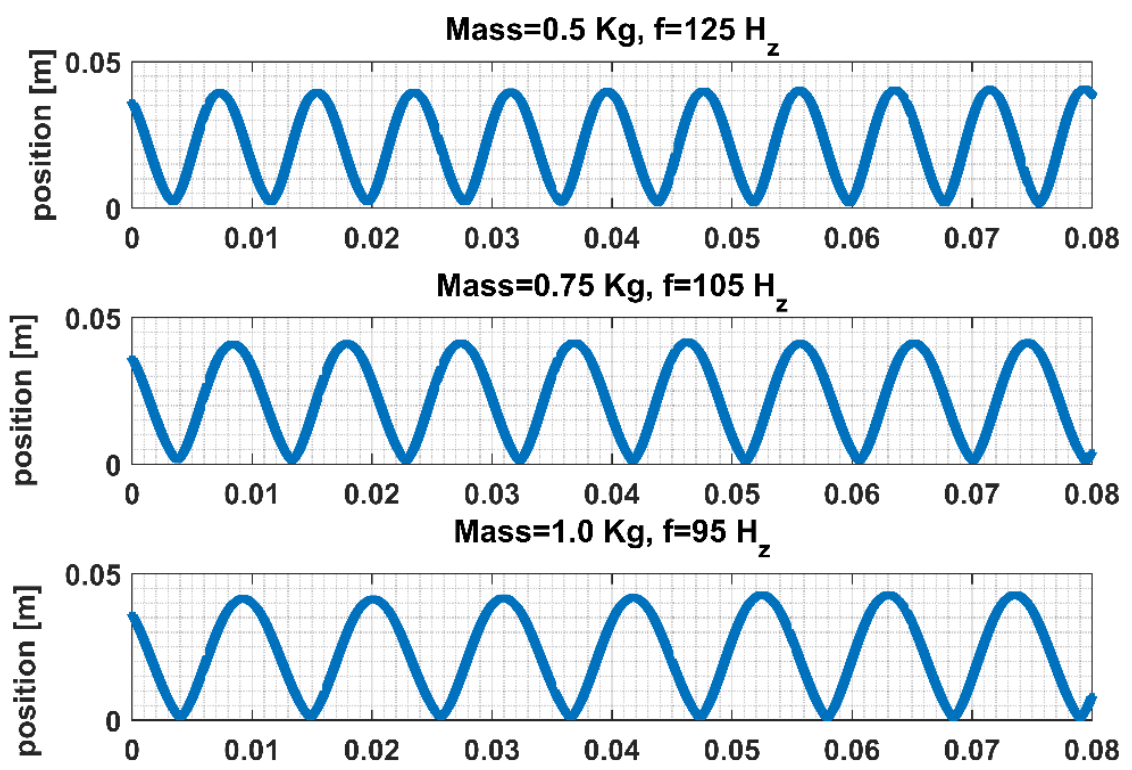

Figure 29 Free-piston engine frequency under different piston mass.

Next, the spring's stiffness is considered, many studies showed that, the engine will operate at its natural frequency where springs stiffness can only be varied over a limited range. The stroke length is strictly limited by the need of a sufficient compression and expansion; hence, there will likely be limitations in the frequency control possibilities of the engine using this parameter [26]. In this study, the engine frequency has been tested under three different spring`s stiffness values. The results are shown in Figure 30 in a decreasing order of the stiffness. Figure 30 indicates that the frequency has an inverse relation to the spring's stiffness. The increasing in the system frequency is obvious when high stiffness is applied. The greater stored energy in the springs will assist the piston to complete its cycle in less time. The fact that, the springs are useful in enhancing the smoothness of the operation can be detected here as well. However, very high stiffness can lead to performance instabilities due to the rapid compression and expansion within the cycle. 

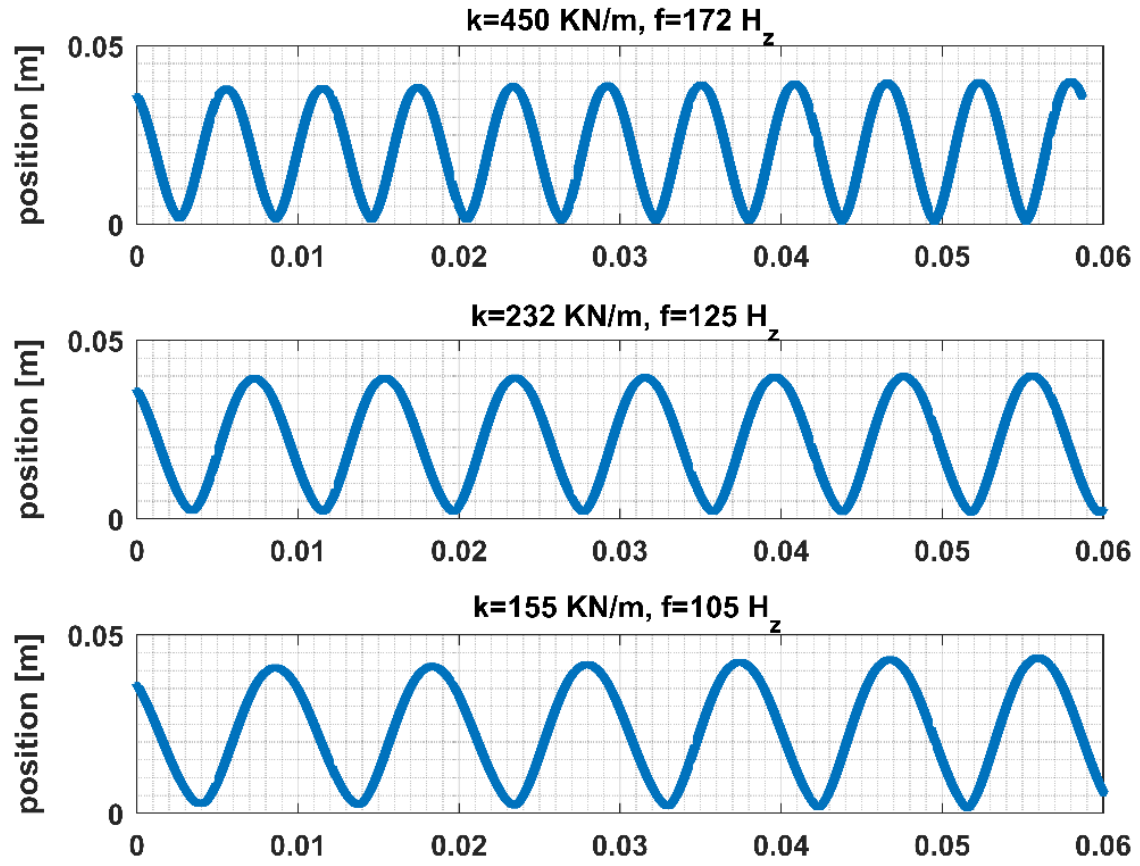

Figure 30 Free-piston engine frequency at different spring stuffiness. 


\section{Chapter 5. Exhaust gas recirculation (EGR) impact on free-piston engine.}

\subsection{EGR and $\mathrm{NO}_{\mathrm{x}}$}

NOx can be produced in the combustion chamber from three sources; thermal NOx, prompt NOx and fuel nitrogen. Thermal NOx refers to NOx formed as a results of the high temperature oxidation $\left(1600^{\circ} \mathrm{C}\right)$ of diatomic nitrogen $\left(\mathrm{N}_{2}\right)$ [128]. This kind of NOx formation is considered as the main source of NOx emissions. The formation rate in the case of the thermal NOx is mainly a function of temperature and the residence time of nitrogen atoms at that temperature $[128,129]$. Prompt NOx is attributed to the reaction nitrogen with free radicals derived from fuel such as $\mathrm{C}, \mathrm{CH}$, and $\mathrm{CH}$. Prompt $\mathrm{NOx}$ occurs early at beginning of the combustion where fixed species of nitrogen like NH is produced [128]. Such species can be oxidized later to form NOx molecules. Fuel nitrogen refers to any NOx molecules produced as a results of burning fuels with nitrogen content like some coal and oil fuel types. Free nitrogen radicals represent the products of fuel nitrogen burning where those radicals ultimately form NOx molecules $[128,130]$. In the current study, all of the NOx analysis refer to the thermal NOx formation due to the high applied temperatures and the nature of $\mathrm{HCCl}$ combustion that will eliminate the prompt NOx form; in addition, natural gas fuel is not a fuel nitrogen source of NOx [128].

Thermal $\mathrm{NO}_{\mathrm{x}}$ emissions in internal combustion engines are mainly in $\mathrm{NO}$ form. $\mathrm{NO}_{2}$ molecules are produced also but with less percentages [130]. The formation mechanism of NO is different from that of UHC and CO (incomplete combustion products); it happens with the proliferation of mixed combustion, concentrations distribution of flame and heat transfer, thus formation reactions is very complex [130]. In addition, the formation of NO molecules is increasing dramatically with temperature increase. The principal stoichiometric reactions, which govern NO molecules formation from molecular nitrogen, are [130, 129]:

$\mathrm{N}_{2}+\mathrm{O} \leftrightarrow \mathrm{NO}+\mathrm{N}$

$\mathrm{N}+\mathrm{O}_{2} \leftrightarrow \mathrm{NO}+\mathrm{O}$

$\mathrm{N}+\mathrm{OH} \leftrightarrow \mathrm{NO}+\mathrm{H}$

Equation (5.4) is an empirical estimation of the NO emissions formation rate. [NO] refers to the molar concentration, and $\left[\mathrm{O}_{2}\right]_{\mathrm{e}}$ and $\left[\mathrm{N}_{2}\right]_{\mathrm{e}}$ denote equilibrium concentrations. NO formation rate is sensitive to the temperature and to the oxygen concentration. The temperature $(T)$ and the oxygen concentration need to be reduced in order to minimize NO formation rate within the combustion process [131]. Fortunately, the chemical kinetics approach has the ability to calculate both of $\mathrm{NO}$ and $\mathrm{NO}_{2}$ levels if their formation equations are included in the fuel mechanism.

$\frac{\mathrm{d}[\mathrm{NO}]}{\mathrm{dt}}=\left(\frac{6 \times 10^{6}}{\mathrm{~T}^{0.5}}\right) \exp \left(\frac{-69,096}{\mathrm{~T}}\right)\left[\mathrm{O}_{2}\right]_{\mathrm{e}}^{0.5}\left[\mathrm{~N}_{2}\right]_{\mathrm{e}} \mathrm{mol} \mathrm{s} / \mathrm{cm}^{3}$

Among the most effective techniques in treating $\mathrm{NO}_{x}$ emissions is using $\mathrm{EGR}$. In this technique, some of the combustion products are recirculated with the intake charge. The combustion products consist mainly of $\mathrm{H}_{2} \mathrm{O}, \mathrm{CO}_{2}$ and $\mathrm{N}_{2}$. EGR has three main effects in reducing $\mathrm{NO}_{x}$ levels. The most advanced effect of EGR is the thermal one where the existed $\mathrm{CO}_{2}$ and $\mathrm{H}_{2} \mathrm{O}$ molecules in the EGR gases have less specific heat ratios than $\mathrm{N}_{2}$ and $\mathrm{O}_{2}$, which found in the pure air [130]. Such feature will allow the gas mixture to absorb the heat energy faster than when no EGR is implemented, therefore; the overall bulk temperature will be decreased [129]. The second effect of EGR is the dilution effect on the gas mixture. The addition of the EGR will be on the expense of the $\mathrm{O}_{2}$ and the fuel concentrations and therefore this will reduce the number of active species since $\mathrm{CO}_{2}$ and $\mathrm{H}_{2} \mathrm{O}$ are inert gases. Typically, lower fuel and $\mathrm{O}_{2}$ concentrations 
means lower HRR since the HRR is proportional to the fuel-air quantity in the combustion chamber [130, 132]. The last effect of EGR applying is the chemical effect done by the presence of $\mathrm{CO}_{2}$ and $\mathrm{H}_{2} \mathrm{O}$ molecules; those molecules have the tendency to restrict the other species reactions since they are more stable even at high temperatures. Therefore, a reduction in NOx formation is expected as $\mathrm{CO}_{2}$ and $\mathrm{H}_{2} \mathrm{O}$ are slowing the combustion reactions rate [130].

Many studies indicated that EGR could have some advantages for the system performance by enhancing the combustion process of the fuel-air mixture. EGR can enhance the system performance by increasing the system thermal efficiency and reduce the cyclic variations within the engine operation [132]. In the current study, the impact of using EGR on the performance and $\mathrm{NO}_{x}$ formation has been investigated.

\subsection{EGR concept}

There are many definitions in the literature for the EGR percentage. One formula for EGR percentage describe the ratio between the EGR gases to the fresh charge mixture only [133].

$$
\operatorname{EGR}(\%)=\mathrm{m}_{\mathrm{EGR}} /\left(\mathrm{m}_{\mathrm{f}}+\mathrm{m}_{\mathrm{air}}\right) \times 100 \%
$$

Where $m_{f}$ and $m_{\text {air }}$ represent the fuel and air mass respectively. However, EGR can be defined based on the volumetric ratio of the recycled gas to the total gas mixture. In this case, the EGR percentage can be up to $50 \%$ of the total exhaust gases [133].

$$
\mathrm{EGR}(\%)=\mathrm{V}_{\mathrm{EGR}} /\left(\mathrm{V}_{\mathrm{air}}+\mathrm{V}_{\mathrm{f}}+\mathrm{V}_{\mathrm{EGR}}\right) \times 100 \%
$$

An alternative EGR formula can be the percent of the recycled exhaust gas to the total intake charge mixture as in following equation.

$\operatorname{EGR}(\%)=\mathrm{m}_{\mathrm{EGR}} / \mathrm{m}_{\mathrm{tot}} \times 100 \%$

In this equation $m_{\text {tot }}\left(m_{\text {tot }}=m_{f}+m_{\text {air }}+m_{E G R}\right)$ is the total summation of the fresh air-fuel mixture and the EGR gas. Based on this formula up to $30 \%$ of the exhaust gas can be recirculated [133]. The formula presented in equation (5.7) has been used to represent the EGR in the current study as shown in Table 4. Each gas of in total charge mixture is presented by its actual percentage. For example, at the case of $E G R=10 \%$, the $\mathrm{CH} 4$ presents $72 \%$ of the fuel mass, N2 presents $69 \%$ of the air mass and $\mathrm{CO} 2$ presents $1.55 \%$ of the EGR mass.

\begin{tabular}{|c|c|c|c|c|c|c|c|c|c|c|c|c|c|}
\hline \multirow[b]{2}{*}{ EGR } & \multicolumn{3}{|c|}{ Fuel } & \multicolumn{2}{|c|}{ Air } & \multicolumn{7}{|c|}{ EGR } & \multirow{4}{*}{$\begin{array}{c}\mathrm{P}_{0} \\
\text { (Kpa) }\end{array}$} \\
\hline & $\mathrm{CH} 4$ & $\mathrm{C} 2 \mathrm{H} 6$ & $\mathrm{C} 3 \mathrm{H} 8$ & N2 & $\mathrm{O} 2$ & Fuel-air & $\mathrm{CO} 2$ & $\mathrm{H} 2 \mathrm{O}$ & $\mathrm{N} 2 \%$ & EGR mass & $\phi$ & $\mathrm{T}_{0}$ & \\
\hline$\%$ & $\%$ & $\%$ & $\%$ & $\%$ & $\%$ & mass & $\%$ & $\%$ & & (g) & & $(\mathrm{K})$ & \\
\hline & & & & & & (g) & & & & & & & \\
\hline 0 & 80 & 11 & 9 & 76.7 & 23.3 & 0.01754 & 15.5 & 12.1 & 72.4 & 0 & 1 & 520 & 101 \\
\hline 10 & 80 & 11 & 9 & 76.7 & 23.3 & 0.01579 & 15.5 & 12.1 & 72.4 & 0.001754 & 1 & 520 & 101 \\
\hline 20 & 80 & 11 & 9 & 76.7 & 23.3 & 0.01403 & 15.5 & 12.1 & 72.4 & 0.003508 & 1 & 520 & 101 \\
\hline 30 & 80 & 11 & 9 & 76.7 & 23.3 & 0.01228 & 15.5 & 12.1 & 72.4 & 0.005262 & 1 & 520 & 101 \\
\hline
\end{tabular}

Table 4 Initial conditions and parametrs for all of the EGR cases.

\subsection{EGR with constant intake temperature}

\subsubsection{EGR effect on the engine cycle}

The conditions and parameters that mentioned in Table 4 have been applied within the engine model to study the effect of the EGR on the overall system. Figure 31 presents the piston position versus the 
piston speed at different EGR ratios. The egg-shape profile is clearly noticed for the entire EGR ratios. The profile undergoes some slight changes as the piston start to move from the BDC toward the TDC. As EGR is presented, the velocity of the piston diverges from the no-EGR path as the piston becomes at the middle of the compression stroke. This divergence increases as the piston is closer to the TDC. Once the combustion process starts, a deceleration in the piston speed is observed. The increasing pressure within the combustion chamber forces the piston to slow down. At this point, the piston starts to move in the opposite way along the expansion stroke. In order to return the piston to the same initial point, the alternator adjust the applied load for each EGR case with different load values.

Figure 32 describes the HRR of the combustion process under the effect of the EGR implementation. Two main effects are observed as the EGR ratio starts to increase, the first is the noticeable reduction in the HRR and the second is the change in the ignition delay of the charge. In the case of the HRR, the reduction in HRR is directly proportional to the less fuel-air molecules available for combustion since they are replaced by the EGR molecules. In addition, EGR gases will dilute the entire mixture which will slow down the fuel-air burning rate due to the inert nature of the EGR gases compared to the fuel-air molecules. In the case of the ignition delay, Figure 32 indicates that the ignition time becomes shorter as the EGR ratio increases. The effects of the EGR on the combustion process explain this since the mixture will absorb the heat energy faster during the compression stroke. The presence of the $\mathrm{CO} 2$ and $\mathrm{H} 2 \mathrm{O}$ will advance the mixture temperature to reach higher values before the combustion; therefore, the charge mixture will reach the ignition point earlier. Chemically, $\mathrm{CO} 2$ molecules can delay the ignition due to their inert nature, but in the current case of the free-piston, it seems that the EGR has a counter effect due to the unconstrained nature of the device which allows the achieving of high compression ratios.

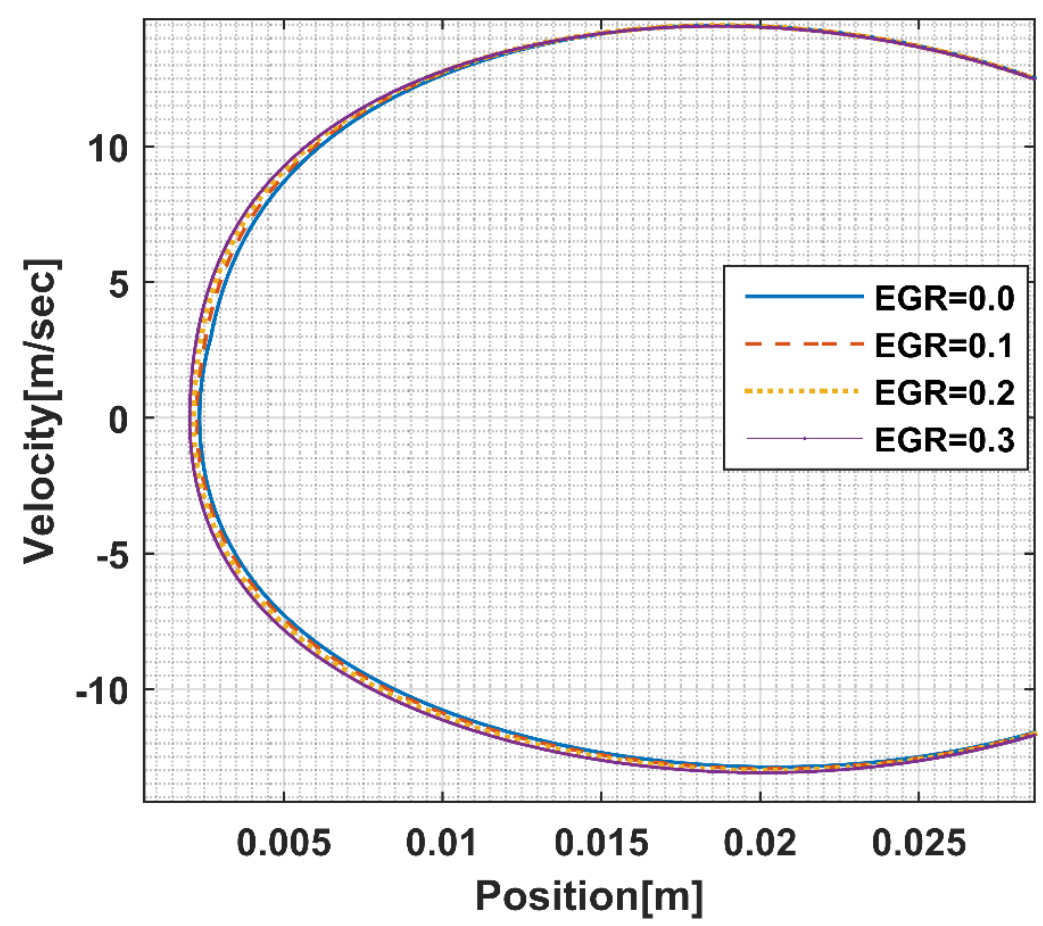

Figure 31 Velocity vs. position at different EGR ratios. 


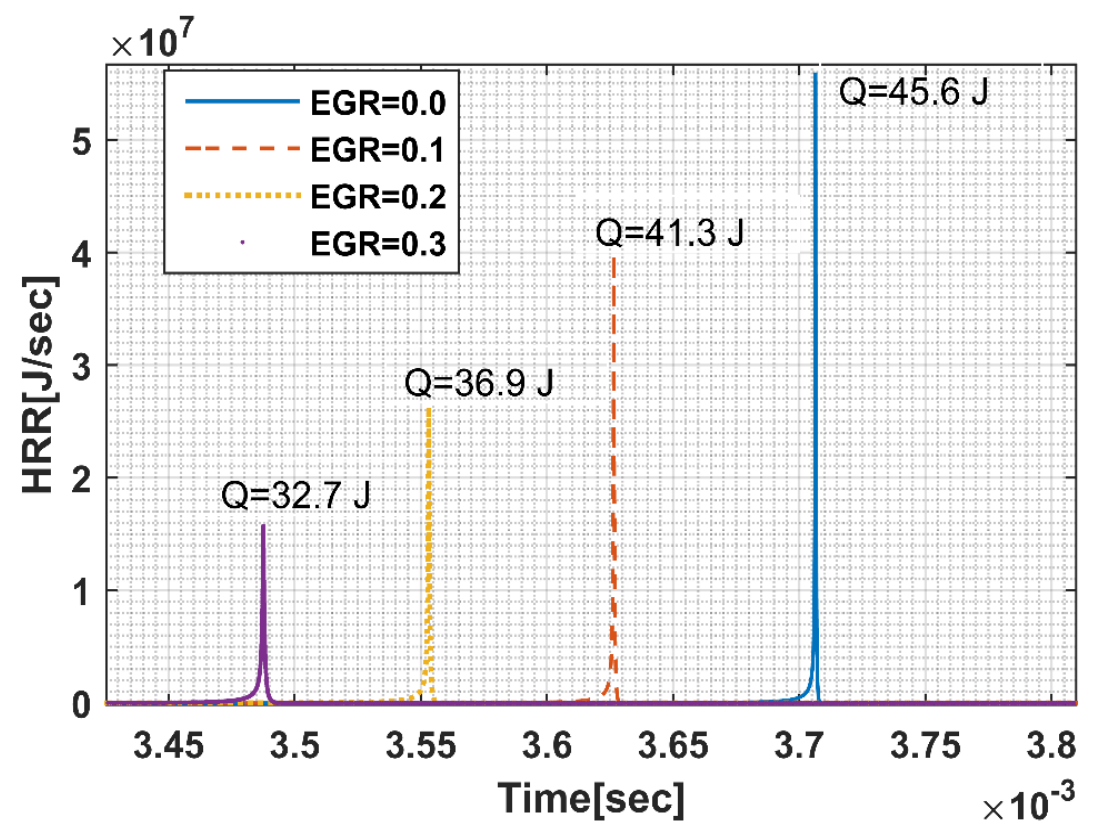

Figure 32 HRR and released energy at different EGR ratios

Figure 33 shows the temperature variation within the combustion chamber at different EGR ratios. The ignition process follows the same trend for all EGR cases where the intake conditions are constant and the alternator load vary with EGR in order to sustain the cycle stability. In all EGR cases, the process can be described as a sudden and rapid step jump in the temperature value near the TDC location. This type of ignition refers to the nature of the $\mathrm{HCCl}$ combustion, which is represented as spontaneous burning of the fuel mixture. The effect of EGR is shown in Figure 33 on both of the self-ignition temperature and on the combustion peak temperature. The self-ignition temperature is controlled by the fuel composition and the time available for the fuel-air molecules to stay under this temperature. The presence of the EGR gases will change the total mixture by shifting it to be more inert due to the $\mathrm{CO} 2$ and $\mathrm{H} 2 \mathrm{O}$ content. In addition, EGR will speed the heat absorption of the mixture during the compression stroke and will allow the mixture to be compressed to higher pressure values. The pressure raise in the cylinder will cause a temperature raise. Based on that, the mixture will require higher self-ignition temperature as EGR is implemented. In the other side, a reduction in the combustion peak temperature is occurred due to the low HRR caused by the effects of the EGR. The peak temperature depends on the available fuel-air amount and on the time available to burn it. As EGR molecules involve in the process at the expense of fuel- $\mathrm{O}_{2}$ molecules, the burning of the fuel will be limited and slower than when no EGR is applied. This important fact that EGR reduce the peak bulk temperature is strongly required in $\mathrm{NO}_{\mathrm{x}}$ emissions treatment. The $\mathrm{NO}_{\mathrm{x}}$ emission reactions (Eq.5.1, Eq.5.2 and Eq.5.3) between the $O$ and $N$ atoms require high temperature levels to be completed. Achieving low temperatures in the combustion chamber can help in reversing those reactions partially and will assist dissolving the NOx molecules. A detailed discussion will be presented later on when the engine $\mathrm{NO}_{\mathrm{x}}$ emissions is presented. 


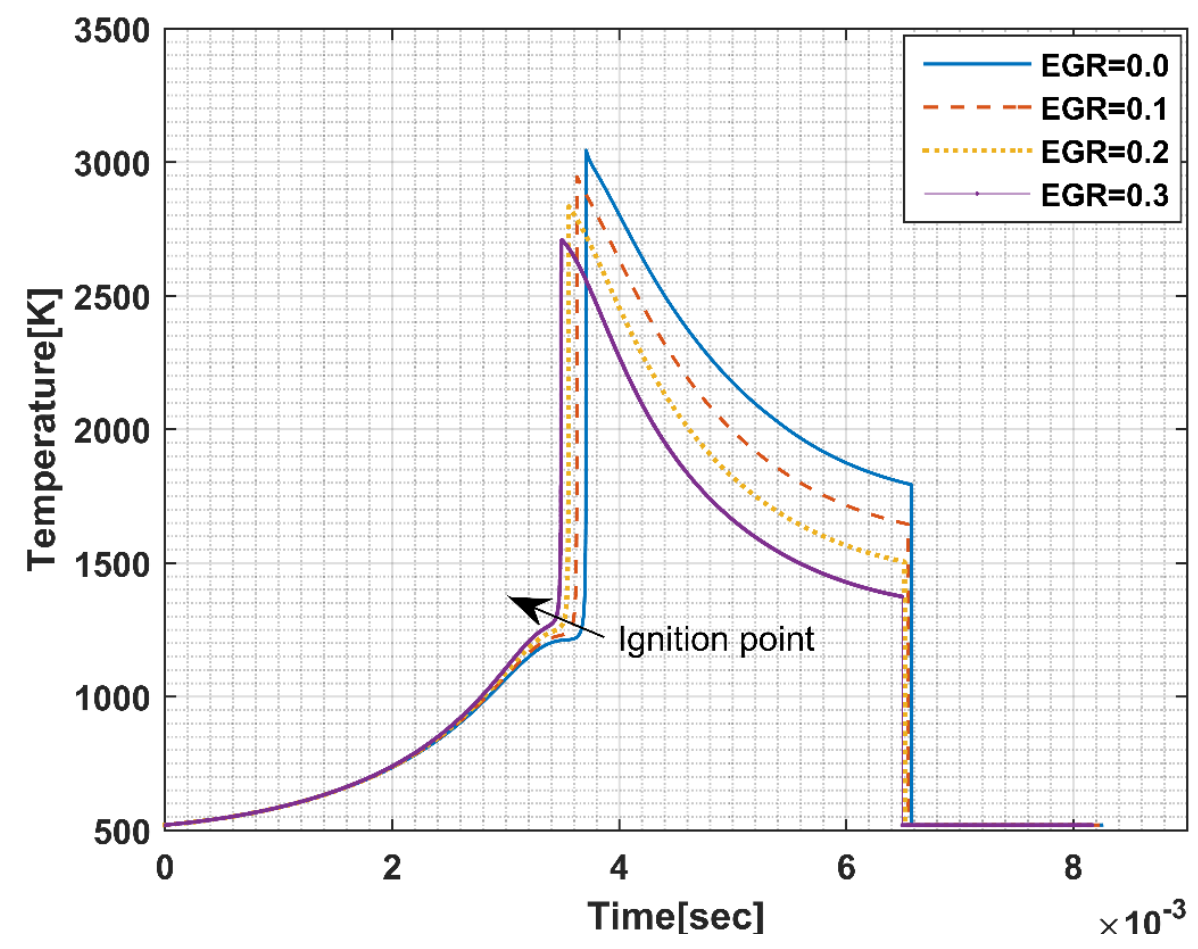

Figure 33 Temperature-time diagram at different EGR ratios.

In Figure 34, the in-cylinder pressure is demonstrated under the influence of different EGR ratios. Under the applied conditions, the figure shows a difference in the ignition pressure where a higher combustion pressure is predicted at higher EGR percentages. The combustion process seems to occur lately (after TDC) when no EGR or low EGR ratios are applied. The pressure profile has two pressure regions in some cases (low EGR); the first is happened due to the impact between the charge molecules during the compression process, while the other happens under the influence of the combustion event. During the first rise, the species that have the weakest branches and bonds will be the most affected since they require less energy to be destructed. In the other case, the main pressure rise region appears as a sudden and sharp change in both of the pressure and temperature. Typically, this region demonstrates the $\mathrm{HCCl}$ ignition phase. In addition, Figure 34 indicates higher peak pressures when EGR ratio is increasing. Many explanations can be provided for this trend in the peak pressure, but the main clear reason is the higher tendency of the charge mixture to be compressed due to its inert nature. By considering the nature of the free-piston engine as well, the compression ratio will increase under such conditions; therefore, the pressure and temperature distribution will vary along the compression stroke based on the applied EGR ratio. Theoretically, the high in-cylinder pressure force leads to high thermal efficiency, however; increasing the pressure beyond certain limits can cause instability in the performance and in some cases may cause engine failure [6]. 


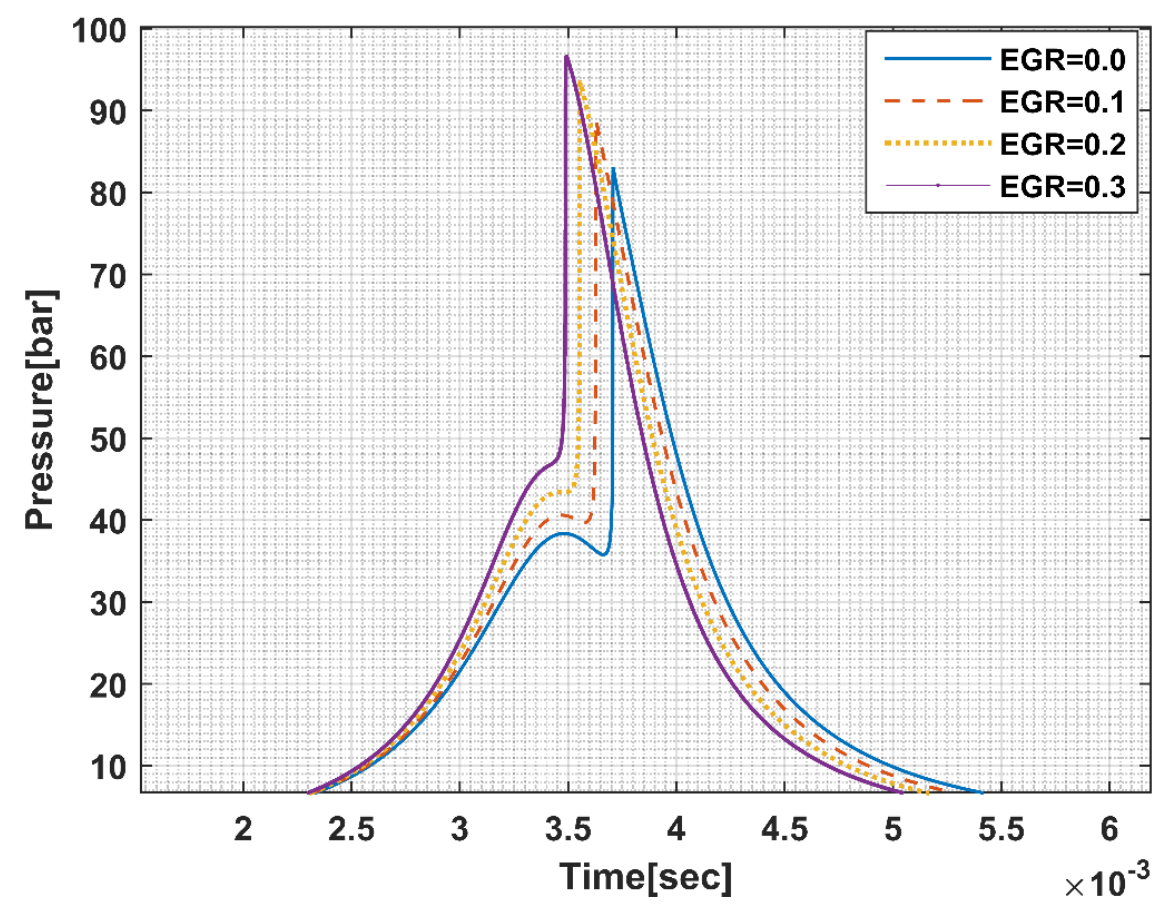

Figure 34 In-cylinder pressure variations at different EGR ratios.

Figure 35 describes the in-cylinder pressure as a function of the piston position. The stoichiometric combustion of the fuel-air mixture passes the two previous mentioned pressure stages. As the EGR ratio increases, the location of the TDC becomes closer to the cylinder head. This change in the TDC location refers to the more pressure required by the mixture to be ignited. In the other side, the disappearance of the first pressure rise is very clear as the EGR ratio increase. Among the reasons of this behavior is the fewer opportunities that the fuel-air molecules will have to be oxidized. An extension for this explanation is that some of the heat need for the fuel-air reaction is absorbed by the $\mathrm{CO}_{2}$ and $\mathrm{H}_{2} \mathrm{O}$. Referring to the first law of thermodynamics, the area under the pressure-position diagram is the work done by the system. This area is increasing as the EGR percentage increase, which explains any noticed increase in overall efficiency. Another important observation is the absence of the adverse work area, which means that the alternator is removing all of the excess energy from the system.

The combustion process can be described also by the temperature position diagram as the one in Figure 36. The figure indicates that the location of the TDC is becoming closer to the cylinder head as the EGR ratio increases due to the delay in the ignition process. However, the peak bulk temperature is decreasing as the EGR increase. It should be noted that the compression stroke is identical for all EGR values while the difference happens in the combustion process and the expansion stroke due to the difference in the ignition time.

Consequently, an increase in the peak pressure is a result of an increase in the compression ratio. This fact is observed in Figure 37 where the CR is illustrated as a function of the EGR ratio. The dynamic clearance volume in free-piston devices allows such variations in the CR since there is no fixed TDC location. At this level of investigation, very high $C R$ values should be avoided to maintain a reasonable engine performance. However, theoretically, as the CR increases, a higher thermal efficiency is expected. In practical life, high CR can lead to engine performance problems such as early combustion event and 
even an engine failure. This fact can be concluded in Figure 37 also, since the trend of the CR is going toward infinity at higher EGR percentages.

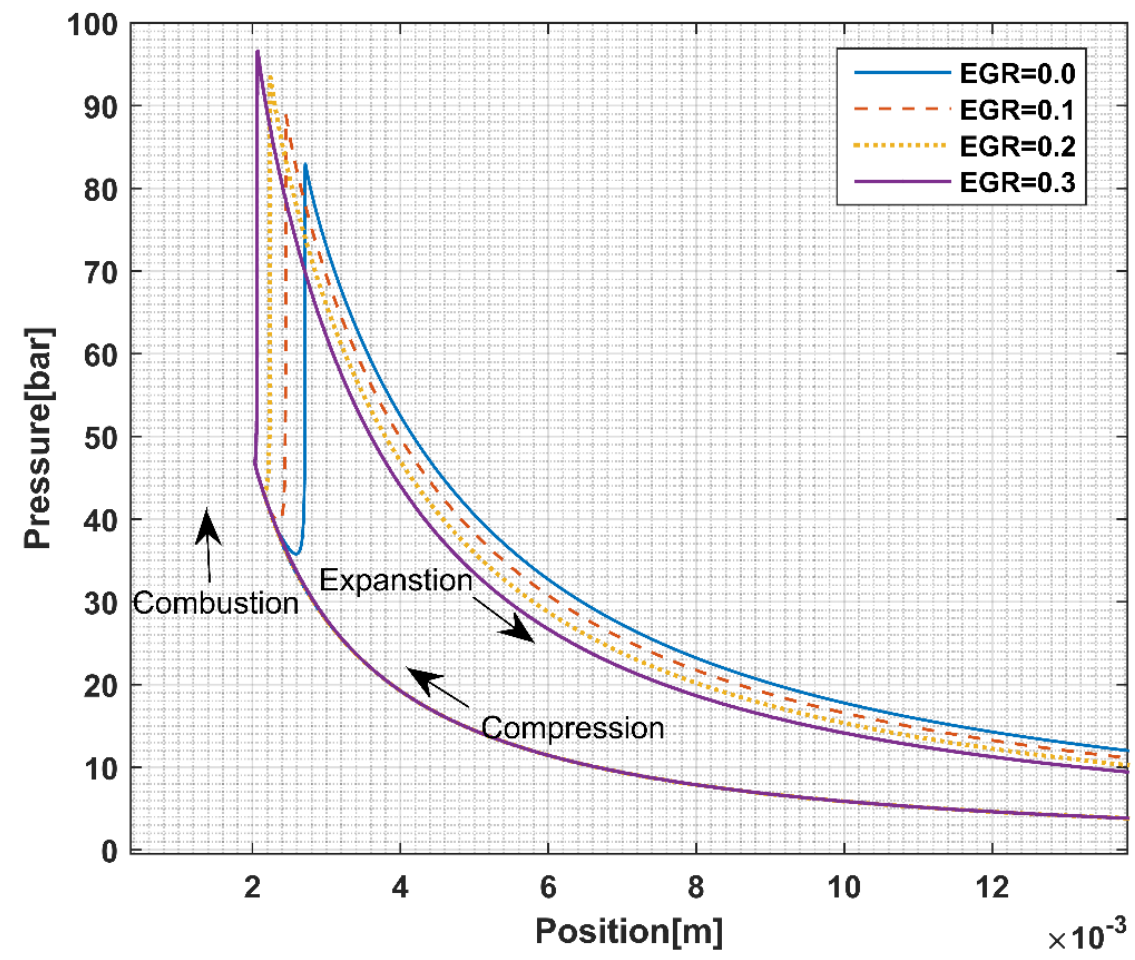

Figure 35 Pressure-Position diagram at different EGR ratios.

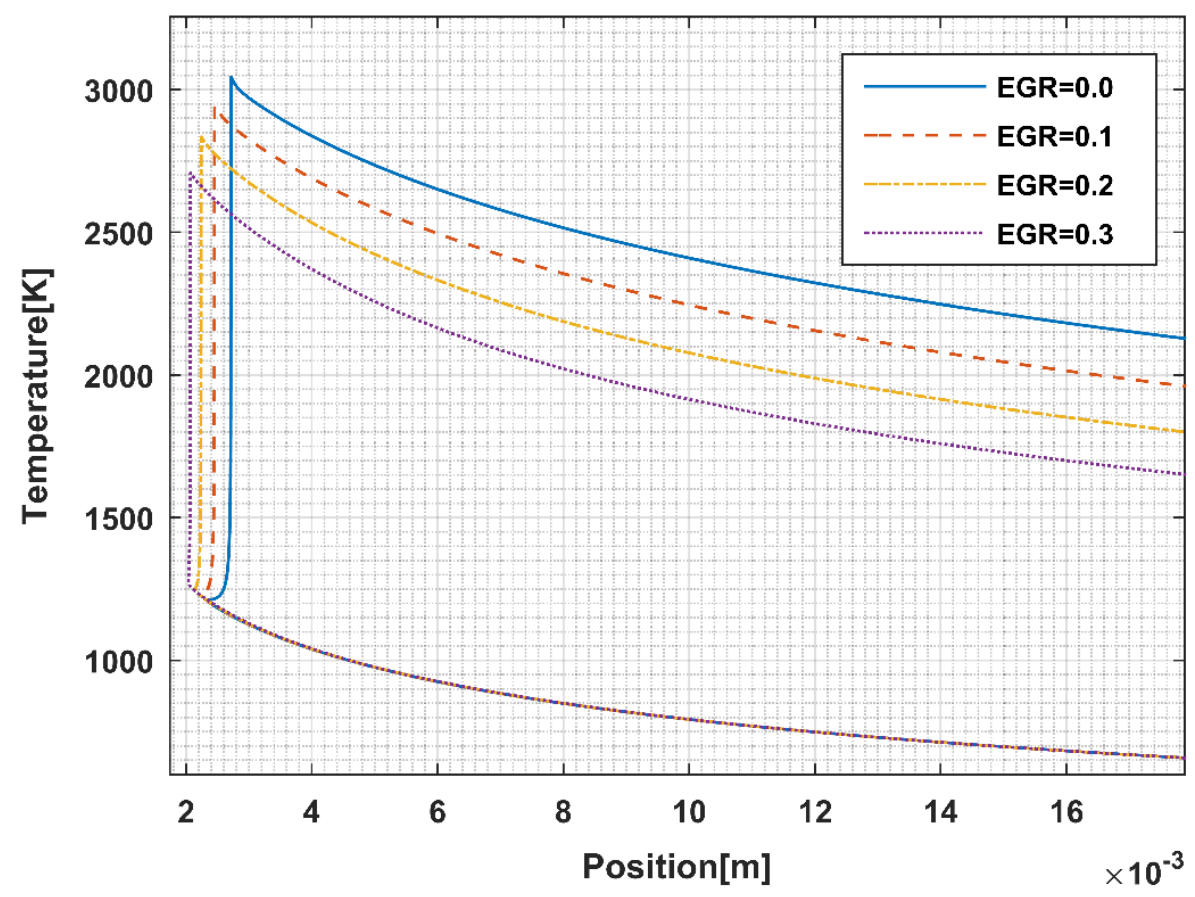

Figure 36 Temerature-Position diagram at different EGR ratios. 


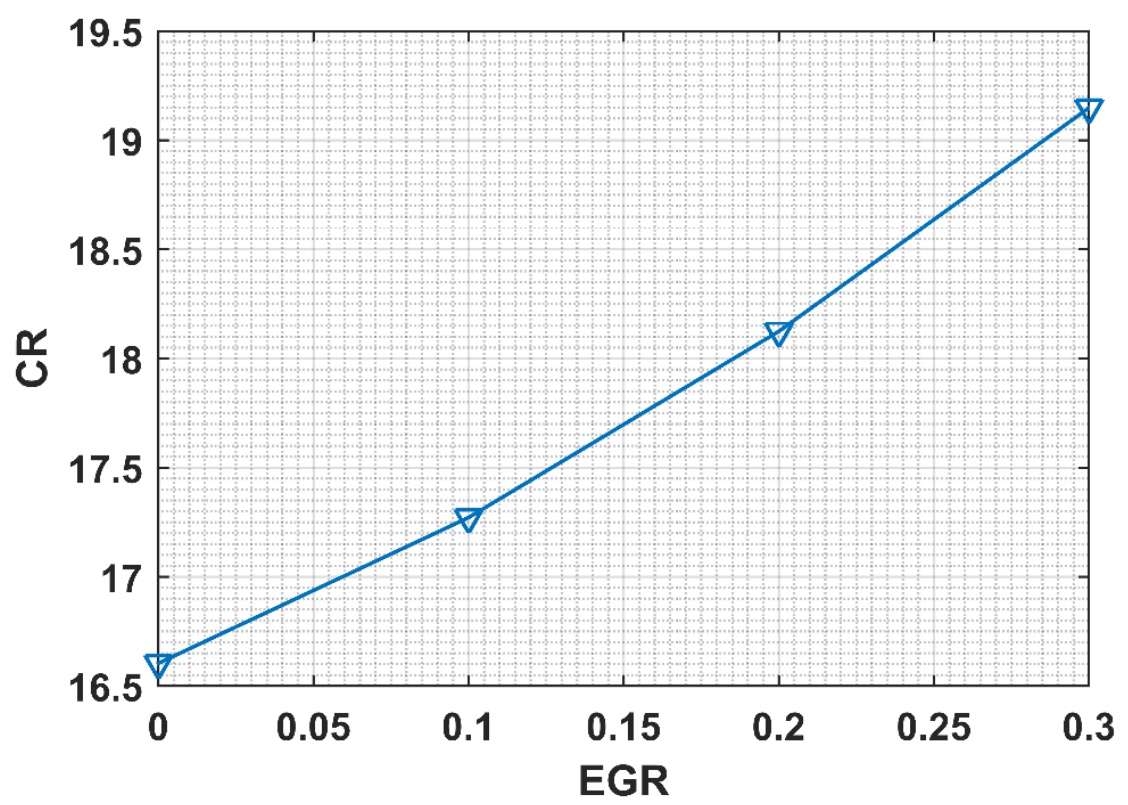

Figure 37 Compression ratio vs. EGR ratio

The work output ratio of the alternator as a percentage of the total input energy is shown in Figure 38. A noticeable increasing in the work output ratio is achieved as higher EGR ratios are implemented. However, a misfire event has been observed as the EGR exceeds the $35 \%$ of the total mass where the model returns a numerical error. It is expected to have incomplete fuel burning at high EGR ratios due to the accumulation of the residual gases that will affect the species reactions. Such type of accumulation will reduce the overall bulk temperature to value less than the self-ignition one which leads to a misfire in combustion chamber. 


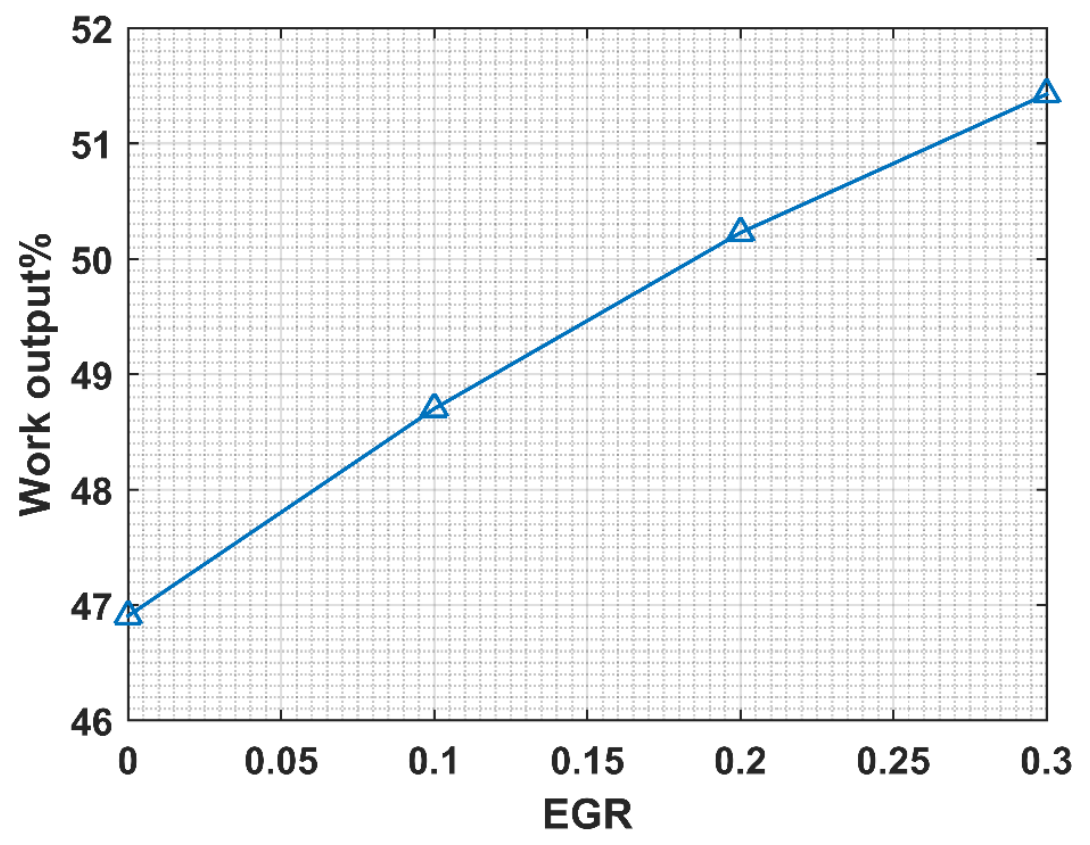

Figure 38 Alternator work output ratio (\%) vs. EGR ratio.

\subsubsection{EGR effects on free-piston engine emissions.}

From environmental perspective, a free-piston engine running under $\mathrm{HCCl}$ conditions has less $\mathrm{NO}_{x}$ emissions outcomes [82]. The nature of the $\mathrm{HCCl}$ combustion gives this feature due to the efficient fuel mixing and burning which are presented. However, $\mathrm{HCCl}$ combustion has limited operation zones and it may show a possible increasing in the engine emissions especially if system modifications are applied [83].

$\mathrm{NO}_{\mathrm{x}}$ emissions are represented by $\mathrm{NO}$ molecules mainly. The species reactions, which describe the formation of $\mathrm{NO}$, have been illustrated before in this section. The $\mathrm{NO}_{x}$ formation of the current engine model is shown in Figure 39. The figure indicates that, even recycling a small percentage (10\%) of the exhaust gases has a significant effect in reducing the thermal $\mathrm{NO}_{x}$ formation. Therefore, a higher reduction can be achieved using higher EGR ratios as long as the operation process is not affected negatively. Under the applied condition, the $\mathrm{NO}_{\mathrm{x}}$ formation follows the trend of the ignition at the start of the formation process. As EGR ratio increases, the formation trend will change from a sudden and sharp $\mathrm{NO}_{x}$ production to a process that is more gradual. Such change in the $\mathrm{NO}_{x}$ formation behavior refers to many reasons such as the dilution effect of the recycled gas. This effect will decrease the combustion peak temperature due to the less fuel-air burning. $\mathrm{CO}_{2}$ and $\mathrm{H}_{2} \mathrm{O}$ molecules, which increase the heat capacity of the mixture, are the motivators of this temperature reduction. This feature in the EGR components reduces the number of the reactions that are responsible about increasing the combustion temperature. EGR has also an impact on the $\mathrm{NO}_{x}$ formation duration by increasing the residence time between the $\mathrm{N}$ and $\mathrm{O}$ atoms. This duration is increased as the EGR percentages; such increase can be explained by the inert nature of the gas mixture as EGR is applied. This plays a significant role in slowing the NOx formation rates. 


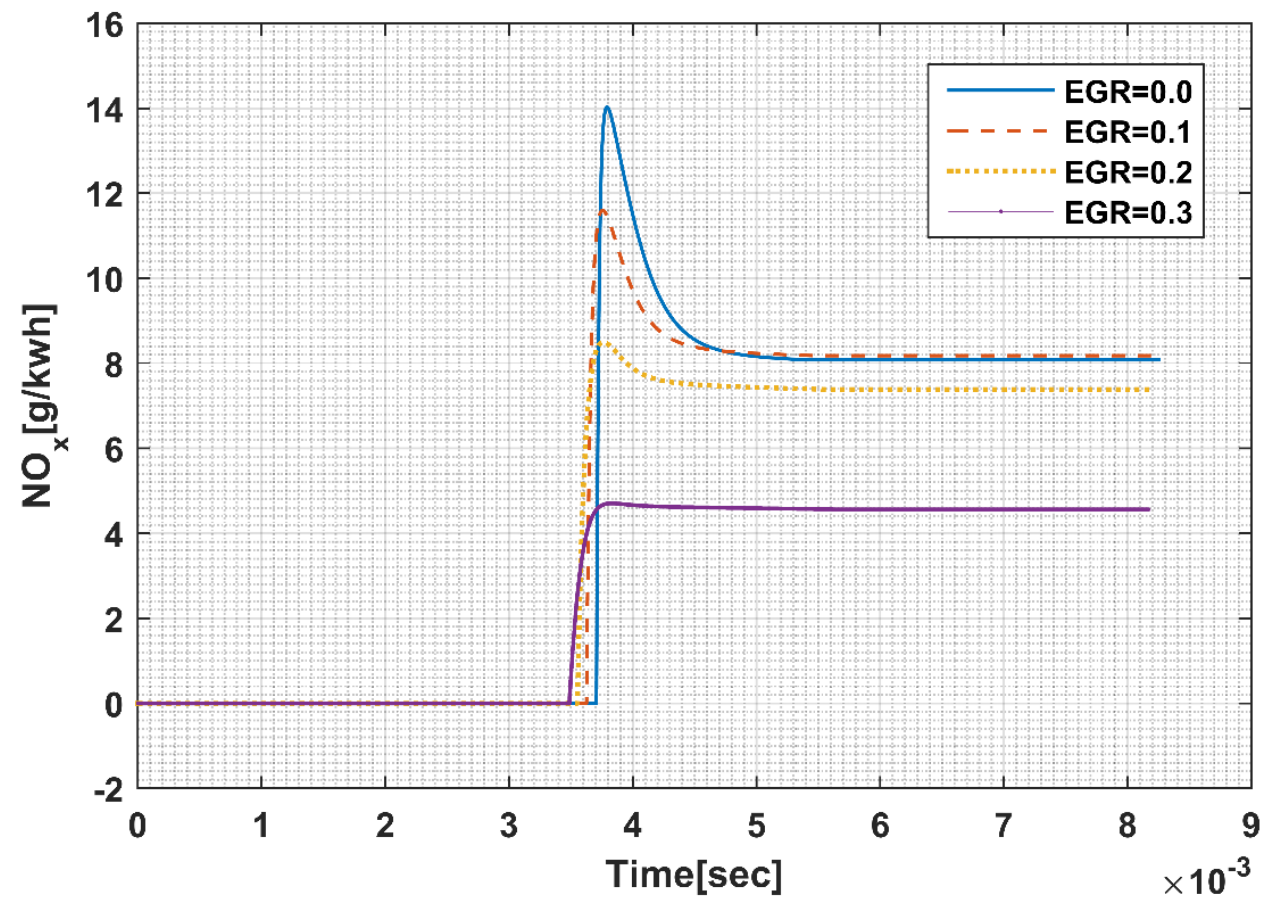

Figure $39 \mathrm{NO}_{\mathrm{x}}$ emissions variations at different EGR ratios.

Although the NOx reduction is noticeable in the current free-piston engine model, but still the emissions levels (EGR<10\%) is considered low compared to spark and compression ignition devices. The nature of the $\mathrm{HCCl}$ combustion process is very fast and rapid, so no enough time to produce high NOx levels is available [134].

EGR is found to affect some other emissions like UHC and CO. These emissions cause many health and environmental problems. For example, $\mathrm{CO}$ is a toxic gas at high concentration. In addition, those emissions have many effects in increasing the greenhouse phenomena. $\mathrm{CH} 4$ (form of UHC) for example can result with more temperature increasing than $\mathrm{CO} 2$ (the main reason of greenhouse effect) [132].

Figure 40 describes the $\mathrm{CO}$ emissions formation under the influence of different EGR percentages. Under the stoichiometric condition, the charges with high EGR percent show lower CO outcomes. The fuel-air combustion of gas mixture indicates that the formation of the $\mathrm{CO}$ emission is concentrated at the ignition time. The sufficient stoichiometric $\mathrm{O}_{2}$ concentration will help in oxidizing the resulting $\mathrm{CO}$ molecules immediately during the ignition. The high temperature of combustion will assist the converting of the $\mathrm{CO} 2$ to $\mathrm{CO}$ molecules as less EGR is applied. Based on that when EGR gases are implemented, fewer chances for the $\mathrm{CO}$ formation will be available. Instead, more time for producing CO2 molecules is available since slower constructive reactions are needed for that. $\mathrm{CO}$ emissions are strong indicator for the UHC emission levels. Typically, higher CO formation is coupled with higher UHC levels, this is expected as the combustion process will include some fuel molecules without complete burning. 


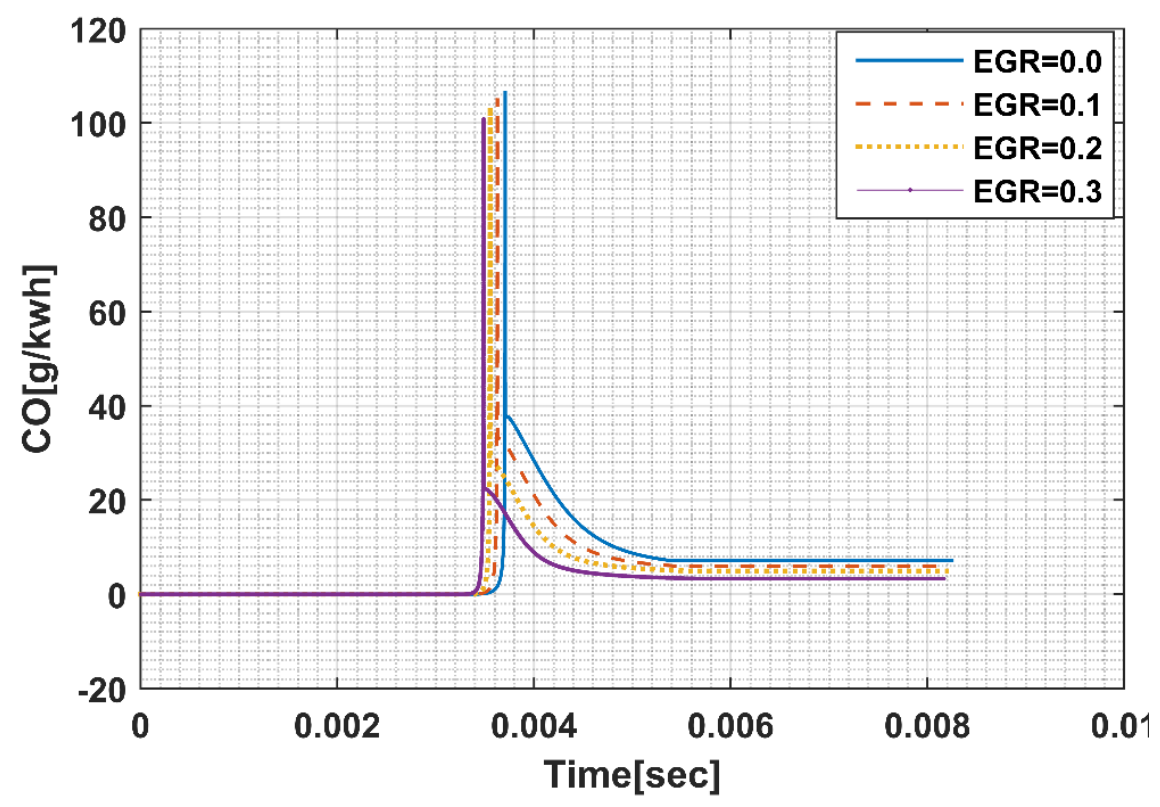

Figure $40 \mathrm{CO}$ emissions variations at different EGR ratios.

\subsection{Hot and cold EGR techniques}

The combustion products have higher temperatures than those at the intake conditions. EGR gases that represent those products will increase the intake charge temperature as they are implemented directly in the combustion chamber. At this case, the EGR process is considered as hot EGR. However, if the EGR gases passed by a cooling stage before the mixing process with the fresh air, it is called cooled EGR [135].The high temperature of the hot EGR promotes the combustion process, it will rise the intake charge temperature and thus improve the thermal efficiency. In the other side, cooled EGR increases the intake charge density of the engine. At the same time, a reduction in the NOx emissions is obtained due to the less achieved combustion temperatures. In contrast, UHC and $\mathrm{CO}$ emissions and cyclic variations are among the problems aligned with cooled EGR [135].

In the current study, the difference between cooled and hot EGR techniques has been investigated. The intake temperature has been changed to simulate the both cases such as the high temperatures present hot EGR features and the low one represents the cooled EGR. A range of temperatures (520-610 K) has been applied while all of the other parameters have been kept the same as in Table 4.

Figure 41 shows the peak engine pressure as a function of both of the EGR and the intake temperature. The peak in-cylinder pressure is increasing as the EGR ratio increases due to the shifting in the ignition time toward the TDC; this effect is observed noticeably as cooled EGR are applied. The increase in the peak cylinder pressure refers to the higher achieved compression ratios. By fixing the EGR ratio and tracking the effect of the intake temperature on the peak pressure, it is found that, the variations in the peak pressure depend also on the applied intake temperature. For low EGR percentages, an increasing in the peak pressure is noticed as the intake temperature increases. However, a reduction in the peak pressure is observed when the intake temperature increases beyond a certain limit ( $>560 \mathrm{~K})$. The temperature of the intake charge, which is controlled by the EGR gases affect the ignition time by advancing the species reaction rate. Therefore, earlier combustion events are observed when high intake 
temperature is applied since the fuel-air mixture will reach the self-ignition temperature earlier. In the case where high EGR percentages are used, the peak pressure will decrease gradually as the intake temperature increases. In general, at low intake temperature (cooled EGR), the EGR has significant effect on the peak pressure by increasing its value as high EGR percentages are applied. However, EGR loses its ability of advancing peak pressure as the intake charge temperature increases due to the early combustion event that occur before the TDC. As mentioned before, high in-cylinder pressure will enhance the thermal efficiency due to the increasing in the pressure force over the piston. On the other hand, excessive pressure values will not be applicable because they will result with engine failure.

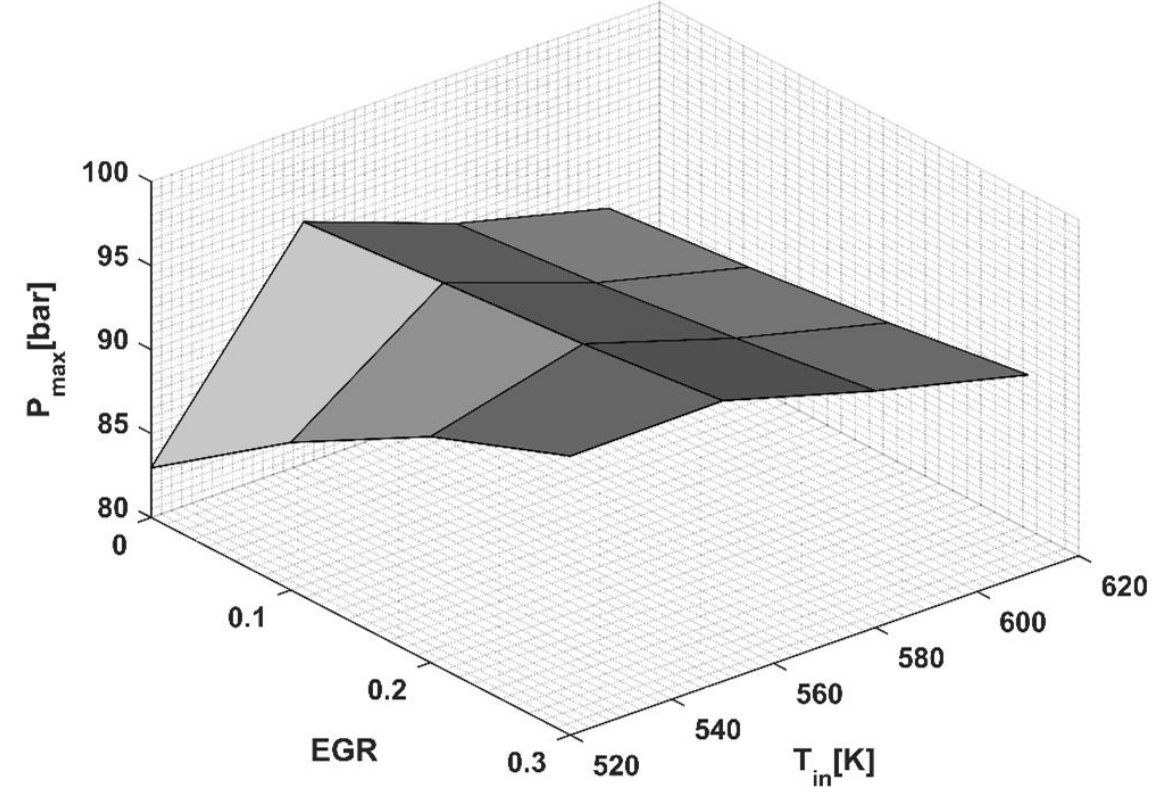

Figure 41 Peak pressure variations at different intake temperature.

Figure 42 describes the CR of the free-piston engine when hot EGR and cooled EGR are implemented. The EGR effect on the CR was discussed before in Figure 37 where high EGR ratios increase the CR strongly by advancing the combustion toward the TDC. In the other side, Figure 42 show that the intake temperature increases the CR for both of the cooled and hot EGR. Such increase in the CR ratio refers to the fact that the ignition event is happening early during the compression stroke. The combustion pressure and the upward moving piston compress the fuel-air mixture toward points closer to the cylinder head. This will decrease the clearance volume, so the CR will be increased. In addition, Figure 42 indicates another important conclusion by showing the independency of the peak pressure on the CR ratio where high CR does not refer to higher peak pressure always. The process is more complicated in the case of the free-piston engine since the system has no fixed ignition point. The location of the ignition plays a significant role in determining the peak pressure whereas the ignition occurs closer to the TDC, the peak pressure will be higher. 


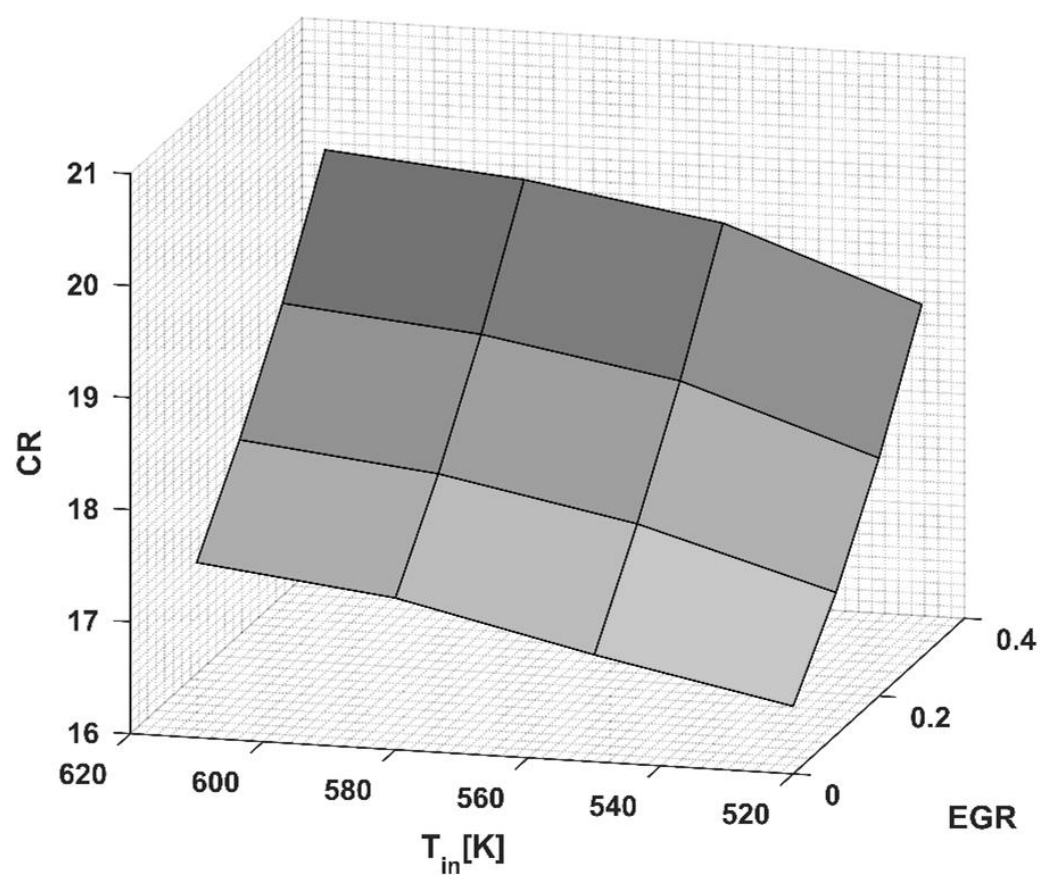

Figure 42 Compression ratio variations at different intake temperature.

Next, the EGR effect on the work output ratio is considered in Figure 43. Under the applied conditions, EGR is found to increase the work output ratio by advancing the in-cylinder pressure. However, excessive EGR will end up with less efficiencies and misfire events in practical operation. In the case of the hot and cooled EGR, Figure 43 shows the work output ratio of the alternator as function of both of the EGR and the intake temperature. The intake temperature effect on the work output ratio depends on the EGR amount where at low EGR ratios; the intake temperature increases the work output ratio slightly within the moderate temperature values. As the intake temperature exceeded the moderate values, a drop in the work output ratio is observed. In the other side, at high EGR ratios, increasing the intake temperature will decrease the work output ratio significantly due to the early ignition events. In general, Figure 43 shows that, high and cooled EGR ratios have the optimal work output percentages. This fact is logical since cooled intake charges has higher charge densities. In addition, the recycled gases is found to affect the ignition time positively, so higher thermal efficiency is obtained.

Furthermore, the difference between hot and cooled EGR effects on NOx emissions has been investigated. The fact that reducing the peak temperature is the motivator of the NOx formation reduction has been studied as well. Figure 44 demonstrates the peak combustion temperature as a function of EGR and the intake temperature. it is shown that, the peak temperature is increasing linearly as the intake temperature increases, this trend is observed for all EGR cases since the high temperatures advance the species reaction rate and therefore the resulted combustion temperature. In contrast, EGR decreases the peak temperature by increasing the heat capacity of the fuel-air mixture, since it has $\mathrm{CO}_{2}$ and $\mathrm{H}_{2} \mathrm{O}$ molecules, which cause that. In addition, the dilution effect caused by those molecules reduces the fuel$\mathrm{O}_{2}$ concentrations and therefore the HRR. Due to the mentioned effects, a lower combustion temperature will result as EGR is implemented in the combustion chamber. The lowest NOx emission levels are obtained when high and cooled EGR percentages are implemented. Again, many limitations will define 
the maximum EGR and the intake temperature values. Engine failure, instabilities in the performance and low efficiencies are examples for such limitations.

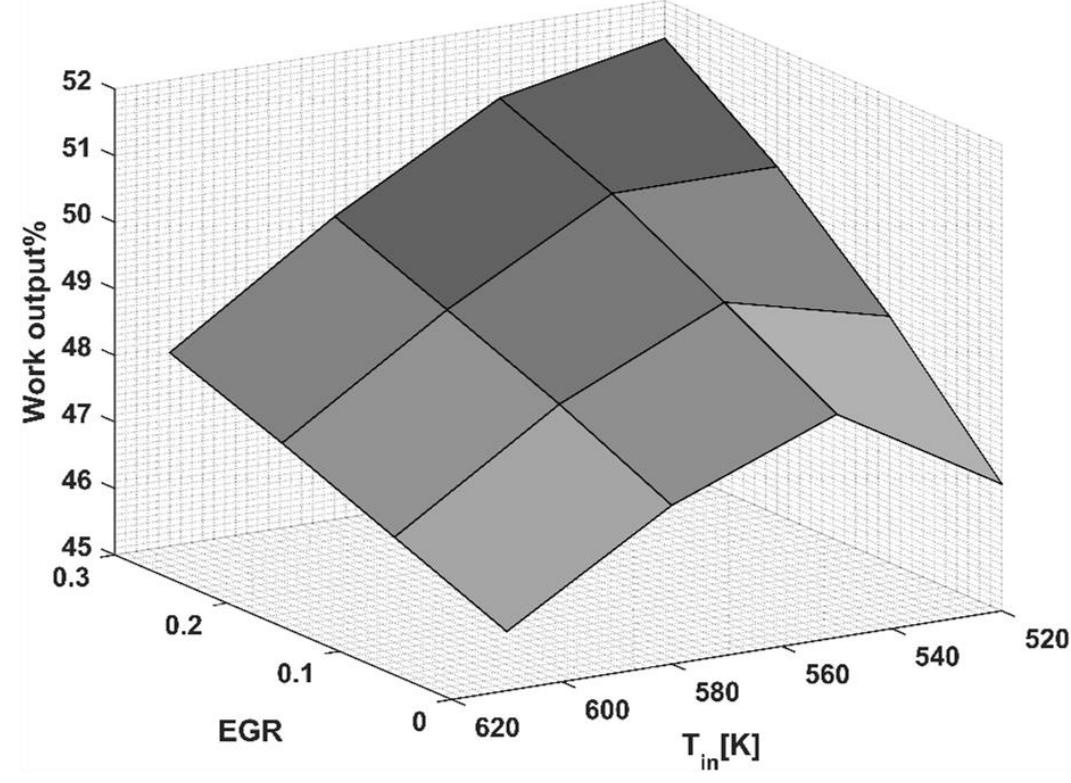

Figure 43 Work output at different intake temperature.

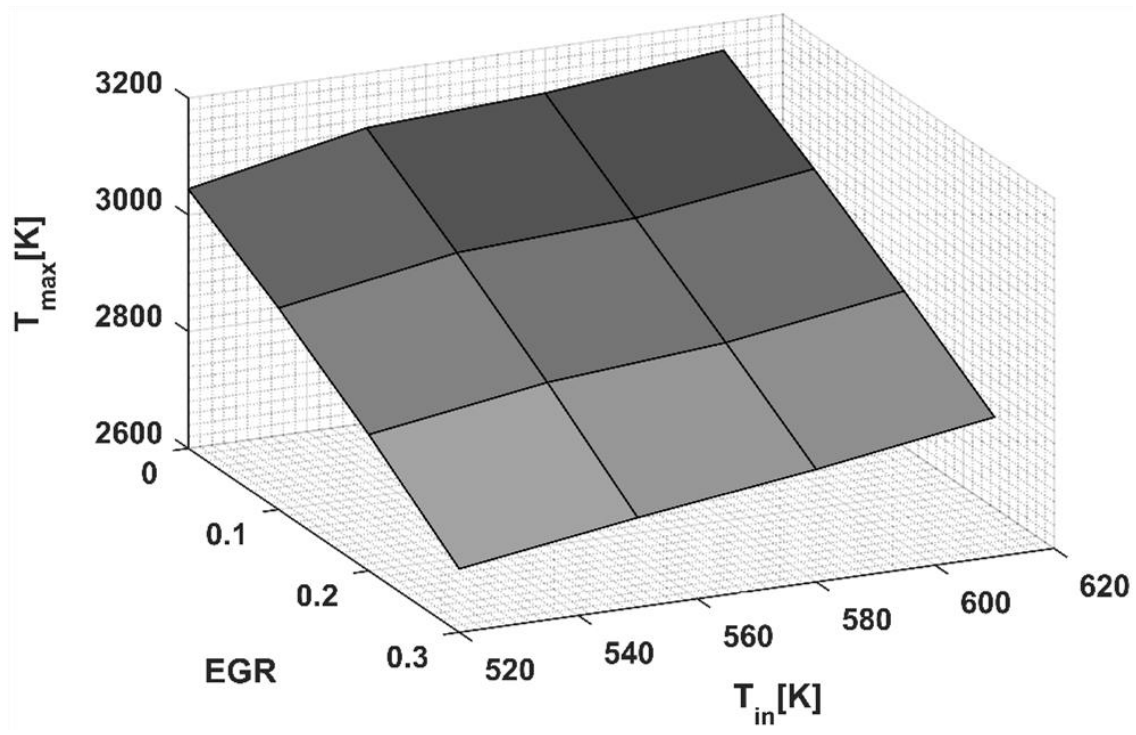

Figure 44 Peak temperature at different intake temperature.

Referring to the main objective of EGR implementation, which is reducing the NOx emissions, the freepiston engine NOx formation is presented in Figure 45 at different intake temperatures and EGR ratios. It is clear that EGR is decreasing the NOx levels aggressively as high percentages are used. The techniques in that are the low peak temperature and the less fuel-O2 concentrations that cause this temperature. Such conditions will affect the reactions rate of NOx production by assisting the decomposition of its molecules. The intake temperature in this case has opposite effect on the NOx production than the EGR effect where it increases the instantaneous reaction rate of the NOx species during the combustion. The 
variable intake temperatures affect the engine performance in different way than the NOx formations. In the case of the engine performance, many engine parameters are affected by the intake temperature like the ignition time and duration. However, in NOx formation, the case is more complicated since the nature of the species reactions will define the NOx productivity primarily. Those reactions seem to have less tendency to be guided by the mixture temperature in a similar way to their responding to the EGR molecules. Later on in this dissertation, the effect of intake temperature will be discussed where the investigations show that the intake temperature has a temporary effect on the NOx formation which is during the combustion, this effect will vanish as the fuel-air burning is completed. Figure 46 explain the relation between the NOx emissions and the EGR percent and peak combustion temperature. The fact that increasing the peak temperature will increase the NOx emission is clearly presented in Figure 46.

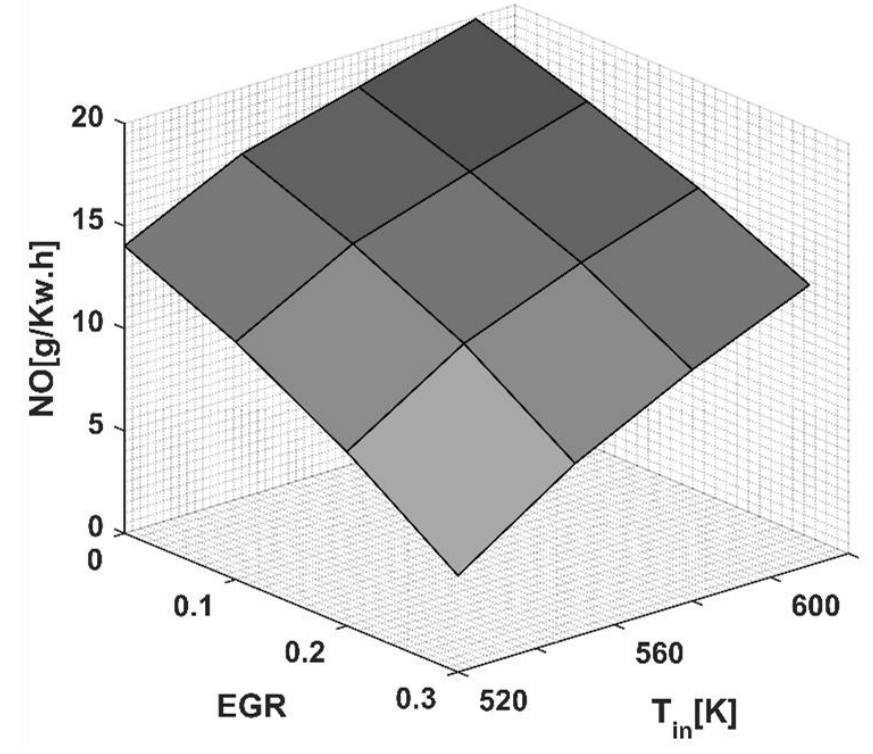

Figure $45 \mathrm{NO}_{\mathrm{x}}$ emissions at different intake temperature.

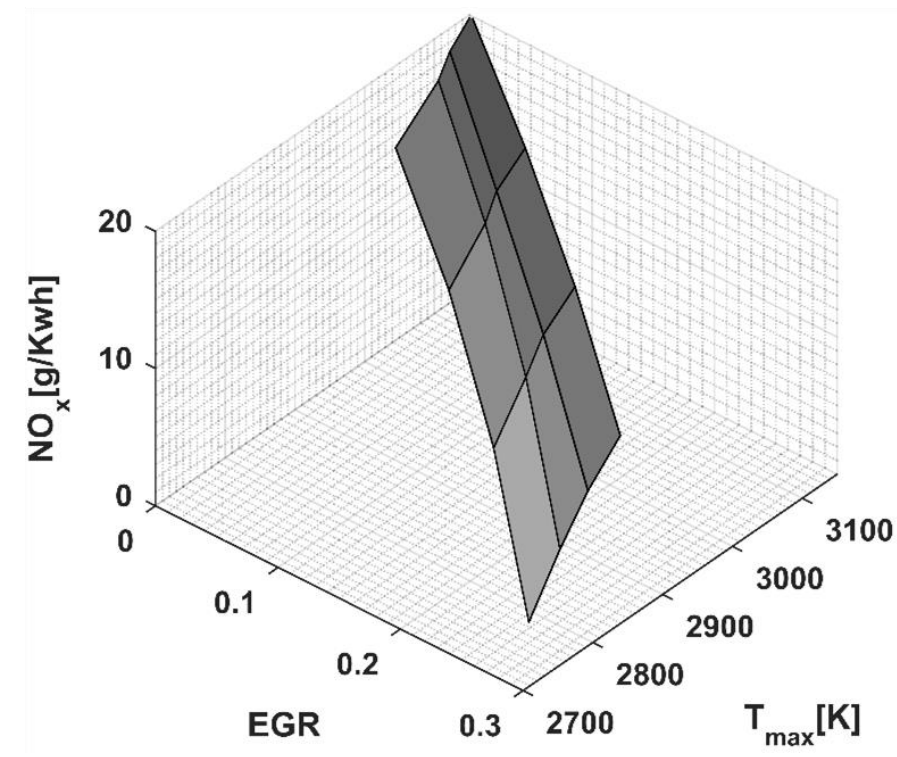

Figure $46 \mathrm{CO}$ emissions at different intake temperature. 
Figure 47 describe the $\mathrm{CO}$ emissions as a function of both of the EGR and the intake temperature, it is clearly described in Figure 47 that the intake temperature encourages the $\mathrm{CO}$ emissions formation regardless the EGR percentage. The intake temperature becomes greater, the species reactions will be motivated, in addition, high temperatures assist the dissolution of the produced CO2 particle to CO ones. The same as in case of the NOx emissions, the intake temperature effect is focused during the combustion time where beyond this time the $\mathrm{CO}$ formation is almost the same for all intake temperature values. By examining the EGR, it is found that EGR has the same effect on reducing the CO emission whatever its temperature. EGR decreases the combustion temperature and therefore will leave less chances for $\mathrm{CO} 2$ decomposition to form $\mathrm{CO}$. the difference between the temperature and the EGR effects refers to many factors. While the intake temperature speeds up the reactions rate of the species, the EGR change the entire mixture composition so more impact is expected like the low fuel-O2 concentrations, which will decrease the burning rate of the fuel. In addition, the abundance of the $\mathrm{CO} 2$ molecules, which will dilute the charge mixture will restrict the $\mathrm{CO}$ formation significantly. In general, to achieve higher engine performance, an optimization process is required when EGR gases are implemented. Many system parameters need to be considered like the required work output, operation stability, durability, and minimum overall emissions levels.

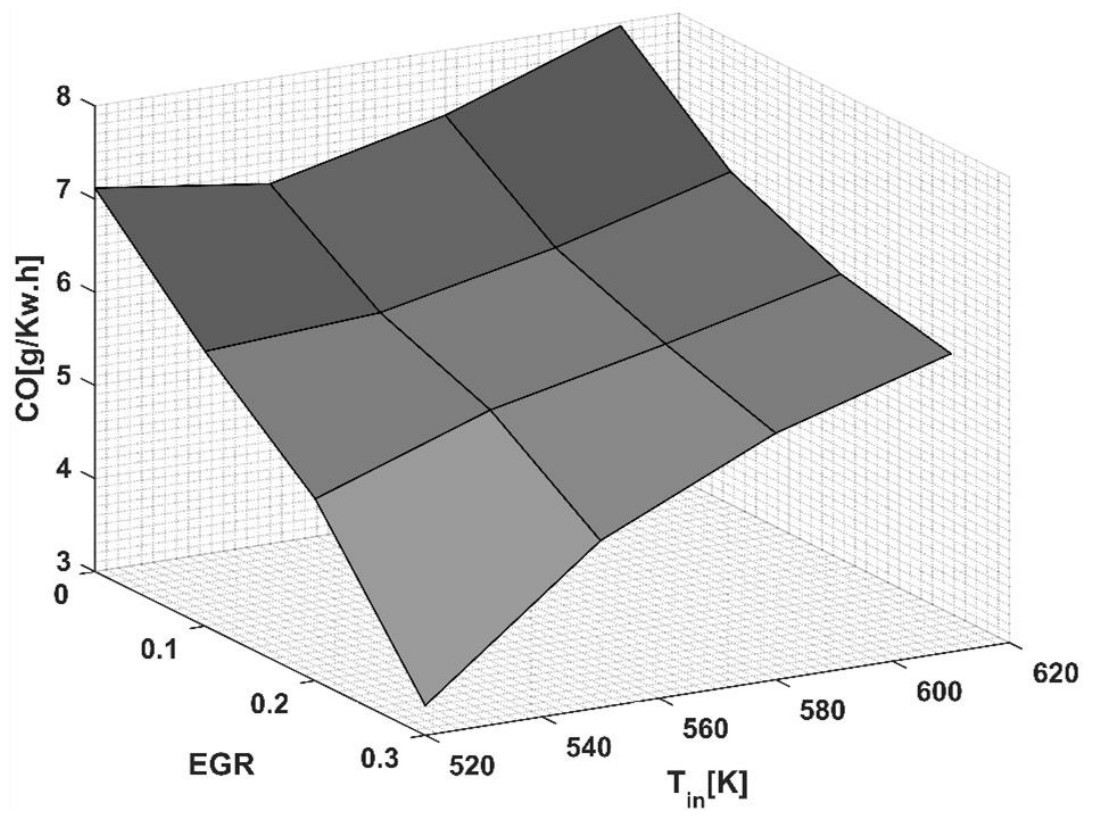

Figure $47 \mathrm{CO}$ emissions at different intake temperature. 


\section{Chapter 6. Intake temperature and equivalence ratio effects}

\subsection{Intake temperature}

In this section, the performance of the free-piston engine has been reported under the influence of the variation in the intake temperature. A range of temperatures has been considered to show the difference between using low and high intake temperature values. The applied parameters and conditions are shown in Table 5.

Table 5 Initial conditions for the applied intake temperatures

\begin{tabular}{cccccccccc}
\hline $\mathbf{T}_{\mathbf{0}}(\mathbf{K})$ & $\mathbf{C H 4 \%}$ & $\mathbf{C 2 H 6 \%}$ & $\mathbf{C 3 H 8 \%}$ & $\mathbf{N 2 \%}$ & $\mathbf{O 2 \%}$ & Air to fuel & $\begin{array}{c}\text { Air-fuel } \\
\text { mass (g) }\end{array}$ & $\begin{array}{c}\boldsymbol{\phi} \\
\mathbf{( K p )}\end{array}$ \\
\hline $\mathbf{5 2 0}$ & 80 & 11 & 9 & 76.7 & 23.3 & 16.95 & 0.01754 & 1 & 101 \\
\hline $\mathbf{5 5 0}$ & 80 & 11 & 9 & 76.7 & 23.3 & 16.95 & 0.01659 & 1 & 101 \\
\hline $\mathbf{5 6 0}$ & 80 & 11 & 9 & 76.7 & 23.3 & 16.95 & 0.01629 & 1 & 101 \\
\hline $\mathbf{5 8 0}$ & 80 & 11 & 9 & 76.7 & 23.3 & 16.95 & 0.01573 & 1 & 101 \\
\hline $\mathbf{6 0 0}$ & 80 & 11 & 9 & 76.7 & 23.3 & 16.95 & 0.01520 & 1 & 101 \\
\hline $\mathbf{6 2 0}$ & 80 & 11 & 9 & 76.7 & 23.3 & 16.95 & 0.01471 & 1 & 101 \\
\hline
\end{tabular}

Figure 48 illustrates the velocity-position diagram of the free-piston engine for a range of intake temperatures that vary from $520 \mathrm{~K}$ to $620 \mathrm{~K}$, this range has been set based on the lowest guaranteed temperature of the ignition. All of the parameters have been held constant except the alternator load, which adjusts the load force to achieve the cycle stability. Figure 48 is zoomed near the TDC point since this region reflect the effect of the intake temperature noticeably. Before the TDC location, the piston velocity within the compression stroke is increasing at high intake temperature values. Such increasing in the piston velocity refers to the low resistance accounted by the fuel-air mixture against the piston motion. As the intake temperature is increasing, the ignition will happen earlier before the TDC location. 


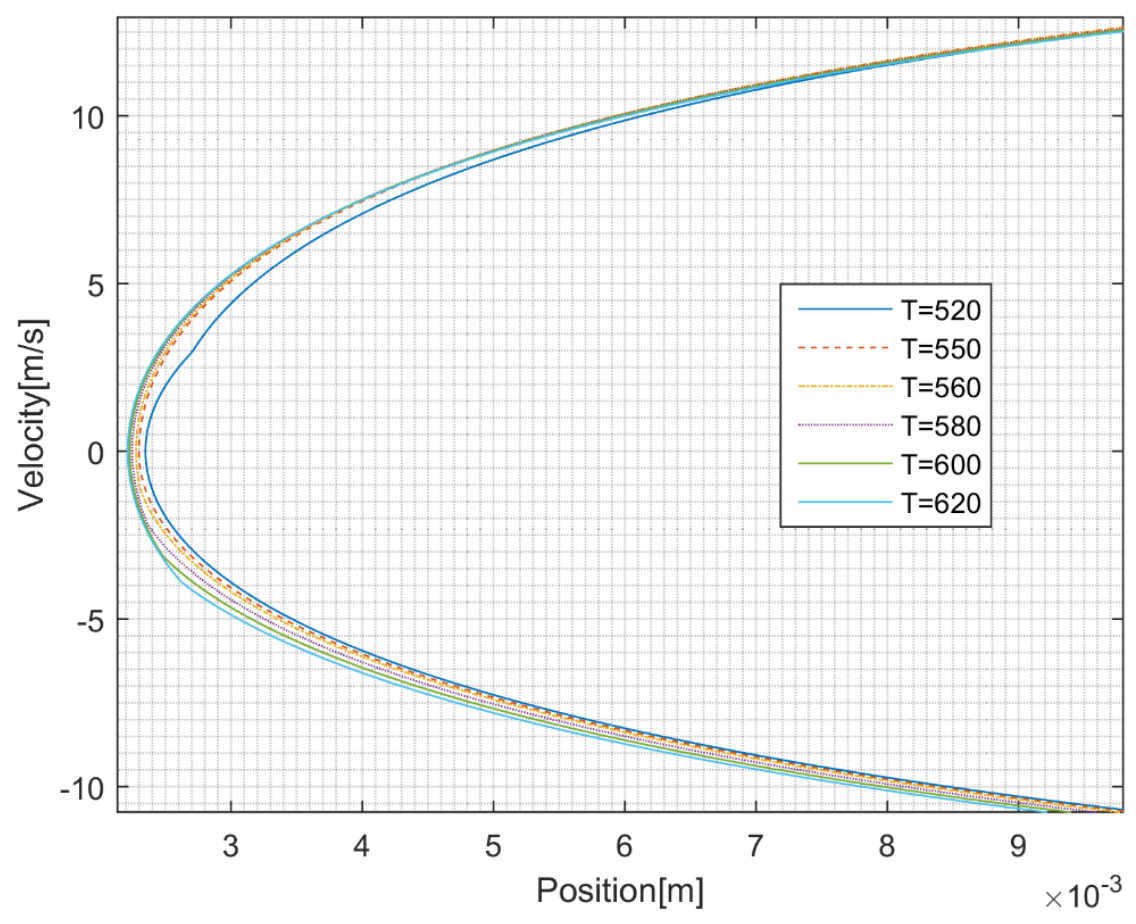

Figure 48 Velocity vs. position at different intake temperature.

The HRR at different intake temperatures is presented in Figure 49. The effect of increasing the intake temperature on the HRR is clear. The HRR increases with increasing the intake temperature, since high temperatures enhance the species reaction rate significantly. Referring to the ignition process, a reduction in the ignition time is noticed as well; this refers to the assistance given by the high charge temperature to the fuel-air burning process. Such assistance will support the mixture to ignite faster by speeding the species reactions rate due to the increase of the impact between the fuel-air atoms. Shifting in the ignition time is observed, that will affect the engine performance by producing different HRR values. Figure 49 describes this clearly, where at low temperatures (520K) the HRR are the lowest; after that, the HRR will start to increase due to the increase in the reactions rate of the species. Finally, when the intake temperature exceeds values of 560K, the HRR will start to decrease down due the early combustion events. 


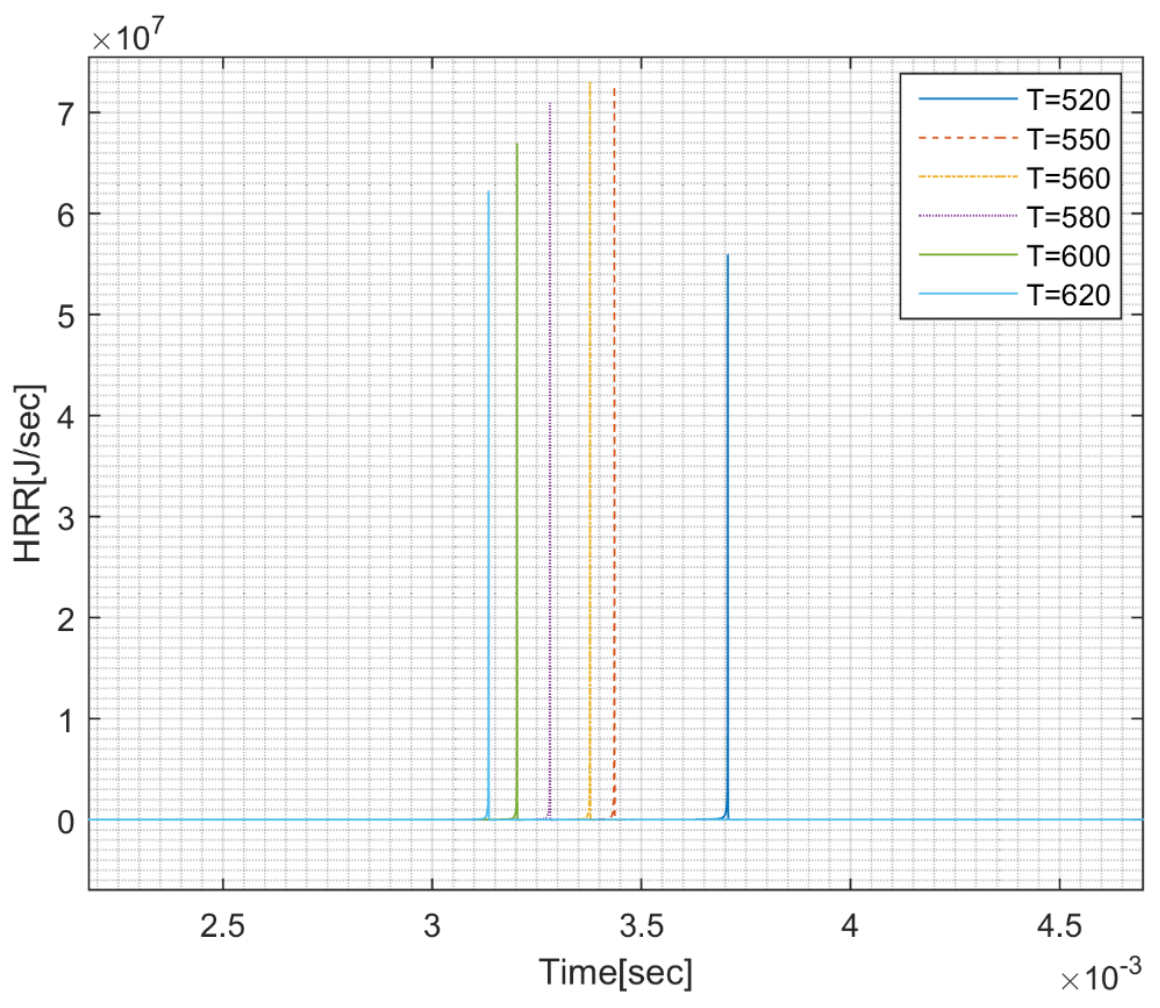

Figure $49 \mathrm{HRR}$ at different intake temperatures.

Figure 50 shows the in-cylinder pressure for different intake temperatures. The delay in the ignition time is clear, as the intake temperature decreases; the ignition is shifting toward points after the TDC location. In addition, cycles that occur at moderate intake temperatures have the highest peak pressures due to the higher HRR and the near TDC ignition events. At higher intake temperatures, the in-cylinder peak pressure drops gradually as earlier ignition events are occurred. The sensitivity of the peak pressure to the intake temperature will affect the alternator work output by mainly affecting the pressure force over the piston. The combustion duration appears to be independent to the intake temperature. It changes under the effect of the fuel-air mixture composition primarily.

Variations in the compression ratio are expected by observing those in the in-cylinder pressure. Figure 51 describes the free-piston engine compression ratio for different intake temperature values. The compression ratio is increasing slightly as the intake temperature increases. This slight increase can be explained by change in ignition location as the intake temperature changes.

By referring to Figure 49 and Figure 51, it is found that, the compression ratio affects the ignition time directly but it has no significant effect on the HRR. The higher compression ratios will accelerate the pressure rising during the compression stroke causing earlier ignition events. However, the species reactions rate will not be affected by any increasing in the compression ratio since they are most likely controlled by the mixture composition. 


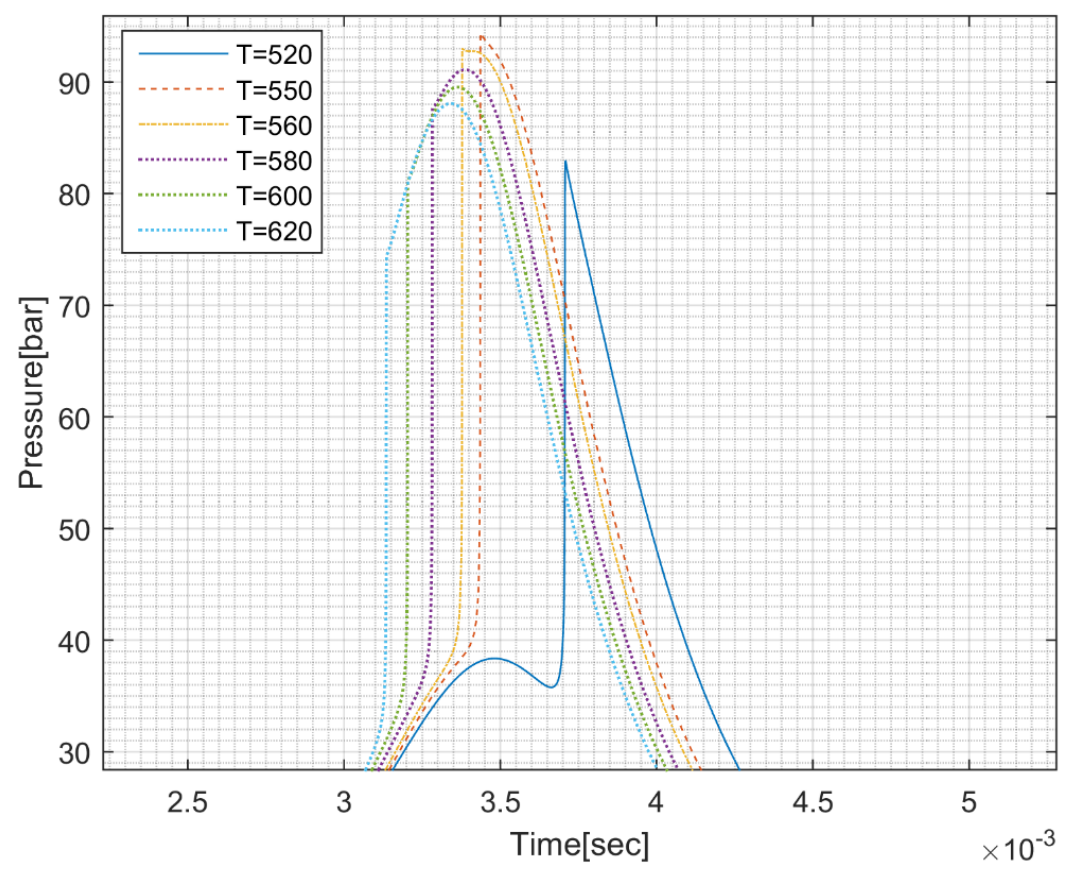

Figure 50 In-cylinder pressure at different intake temperature.

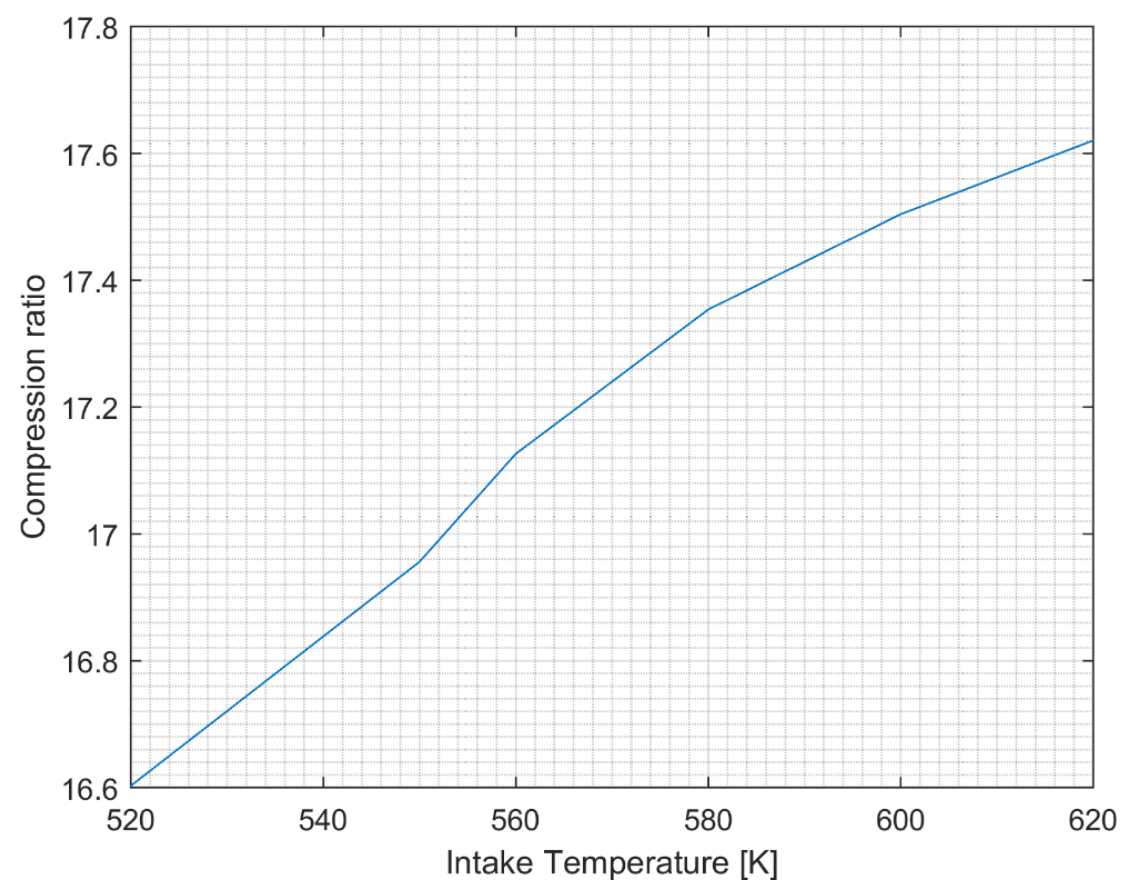

Figure 51 Compression ratio at different intake temperature. 
The free-piston cycle is described in Figure 52 and Figure 53 by presenting the in-cylinder pressure and temperature as a function of the piston position at different intake temperatures respectively. The discussed variations in the ignition time and the HRR can be concluded also from the illustrated diagrams. The TDC location is changing as the intake temperature changes while the ignition event is aligned with the TDC in some cases. For relatively low intake temperatures $(T=520 \mathrm{~K})$, the ignition occurs after the TDC due to the slow reactions rate at the applied temperature. Simply, the resistance to the ignition will increase as the intake charge temperature decreases. However, as the mixture temperature increases this resistance to the ignition will be reduced dramatically. Figure 52 shows another important phenomenon, which is the adverse work zone. As mentioned in the previous chapters, the adverse work appears around the TDC as an inversion of the pressure-position profile. The inversion represents a negative work occurs on the piston as it moves upward. The nature of the $\mathrm{HCCl}$ combustion and the HRR attached with it are the reasons of this work. Basically, the combustion energy held within the piston will be much higher than the pressure force that returns the piston through its expansion stroke; therefore, the piston will end up with more kinetics energy. By increasing the intake temperature, the amount of the adverse work will increase significantly due to the assistance of high temperature in achieving the ignition. This indicates that the piston requires less energy to complete the stroke than the released combustion energy. Chemically, this can be explained by the rapid increasing in the reactions rate which caused by the higher HRR at high temperatures.

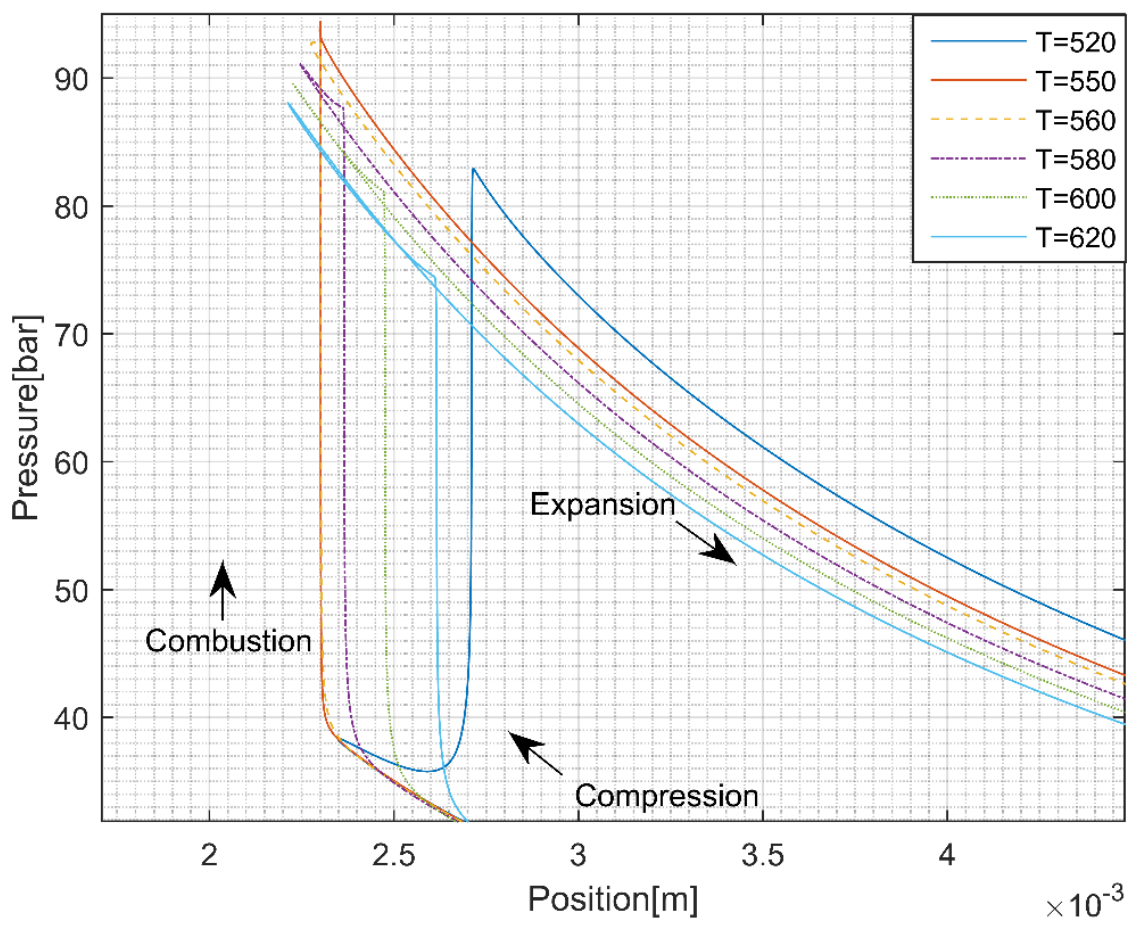

Figure 52 In-cylinder pressure-position diagram at different intake temperature. 


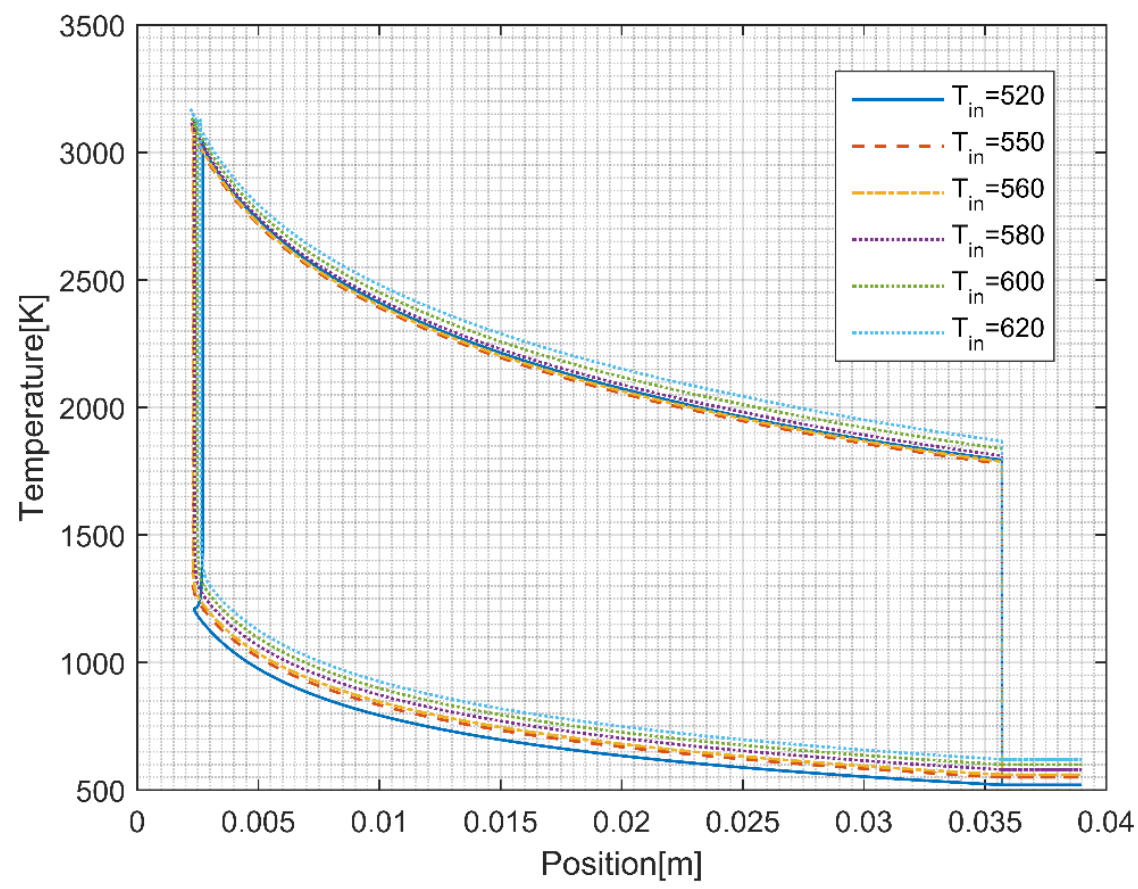

Figure 53 In-cylinder Temperature-position diagram at different intake temperature.

From efficiency perception, the effect of the intake temperature on the work output ratio has been investigated in Figure 54. Typically, the trend of the work output ratio is following the in-cylinder pressure behavior where it is increasing as the intake temperature increases from low to moderate values. After that, it will start to decrease as the intake temperature goes toward higher values. The drop in the incylinder pressure is the reason behind such behavior in the work output ratio. The combustion force that drives the piston will be reduced so less applied force will affect the piston.

Another important parameter that can be affected by the intake temperature variations is the incylinder temperature. Figure 55 demonstrates the in-cylinder temperature at different intake temperature values. Three main observations can be noticed in the figure, the ignition delay, the selfignition and the peak temperatures. The ignition delay time is less when the intake temperature is high and vice versa. Such trend was explained before by the higher reaction rate attached with the fuel-air species. Another observation is the self-ignition temperature where this temperature is increasing for those mixtures with higher intake temperatures. The rise in the self-ignition temperature is logical since when the ignition delay is short, a higher temperature is required to accelerate the species reactions. The peak temperature is also increasing as the intake temperature increases. The higher self-ignition temperatures and the relatively high HRR are the main reasons behind that. 


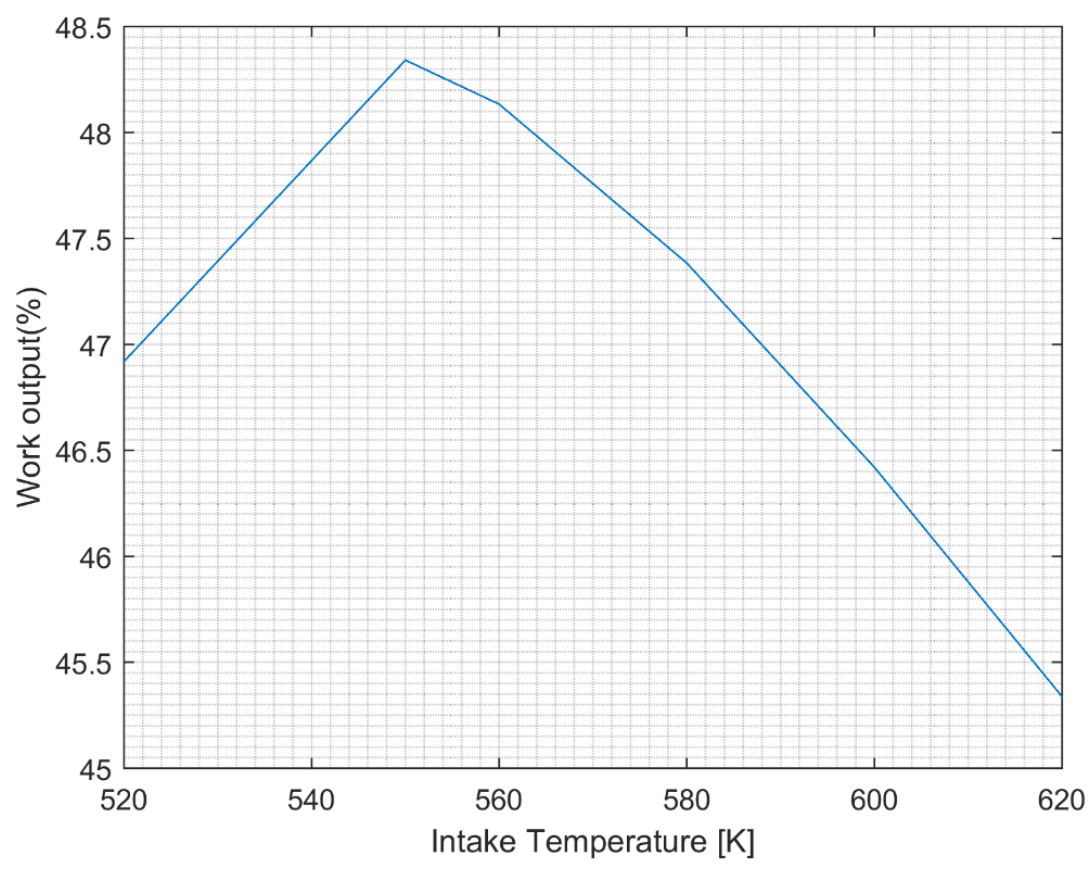

Figure 54 Work output ratio at different intake temperature.

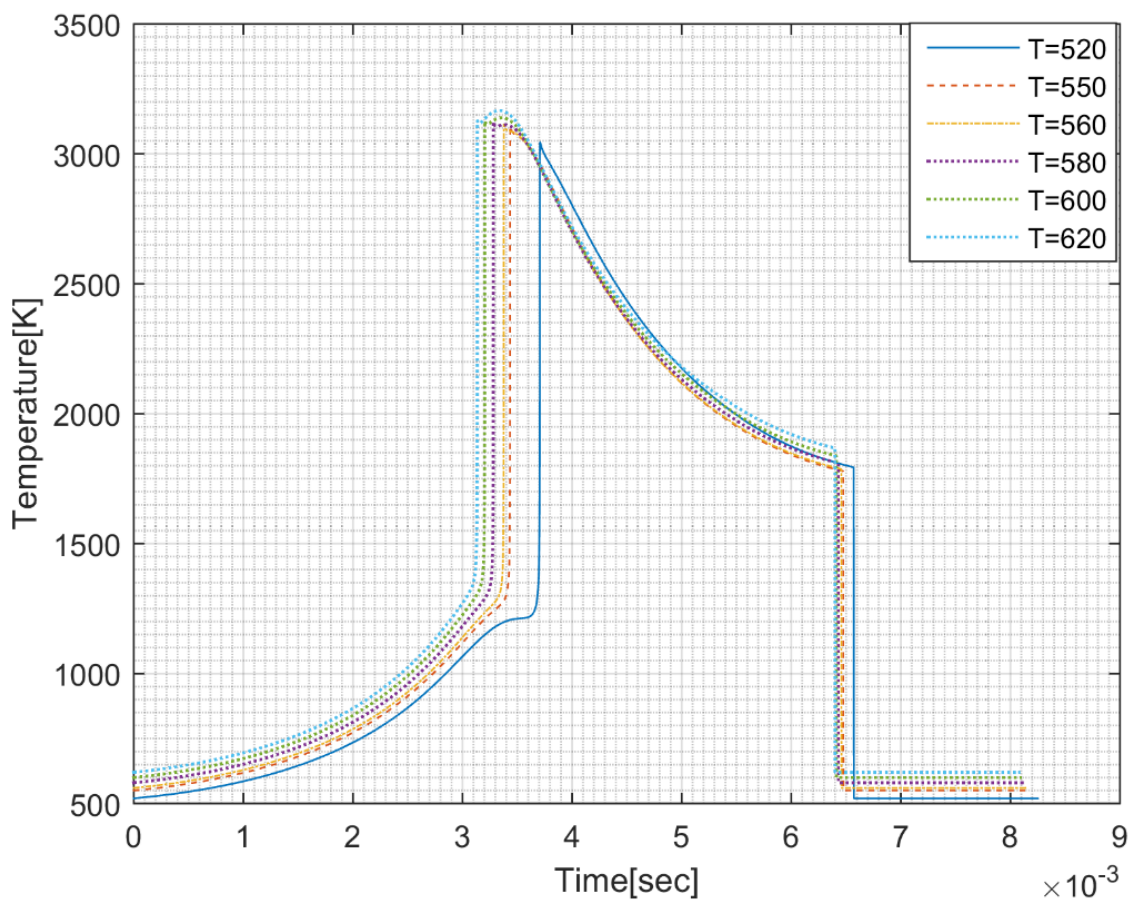

Figure 55 In-cylinder temperature at different intake temperature.

$\mathrm{NO}_{\mathrm{x}}$ formation is illustrated in Figure 56 for different intake temperature values. The instantaneous formation of NOx emissions increases dramatically as the ignition is happening. The increasing in the intake temperature advances the species reactions and this is translated as a higher jump in the $\mathrm{NO}_{\mathrm{x}}$ formation. However, an independency on the temperature is noticed on the $\mathrm{NO}_{x}$ trend as the piston 
passes the TDC zone. Instead, the $\mathrm{NO}_{\mathrm{x}}$ formation rate will be almost the same for all of the different intake temperatures. In fact, the intake temperature affects the instantaneous formation rate but not the overall one. From chemical perception, the fuel-air composition is the same at all different temperatures so the number of the formation reactions will not be affected.

The effect of the intake temperature on the CO formation is described in Figure 57. The same behavior of NOx formation is observed for $\mathrm{CO}$ formation. The figure indicates that the instantaneous formation of the $\mathrm{CO}$ emissions increases as the intake temperature increases, but once the burning process is completed; this rate will be almost the same for all the intake temperatures. In general, the intake temperature effect is focused on the instantaneous reactions rate while the overall rate is not affected by the intake temperature.

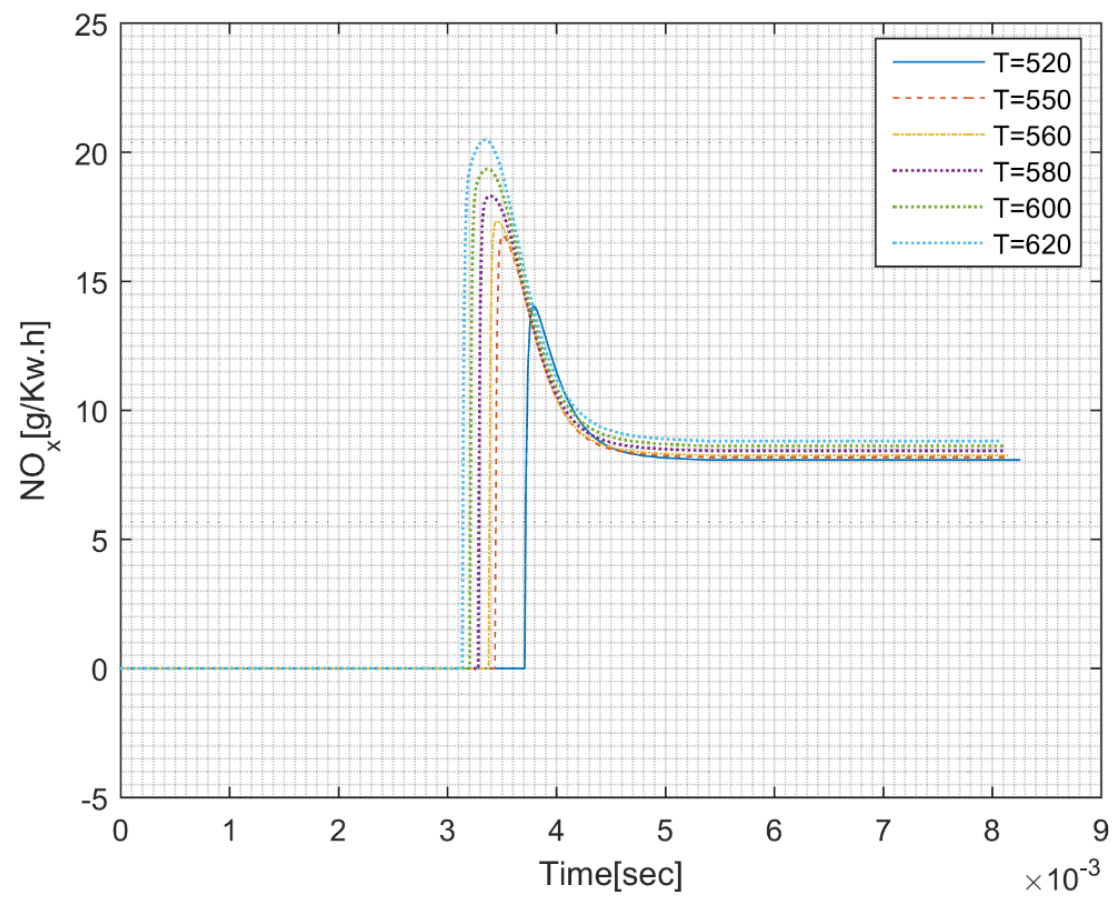

Figure $56 \mathrm{NO}_{\mathrm{x}}$ emission at different intake temperature. 


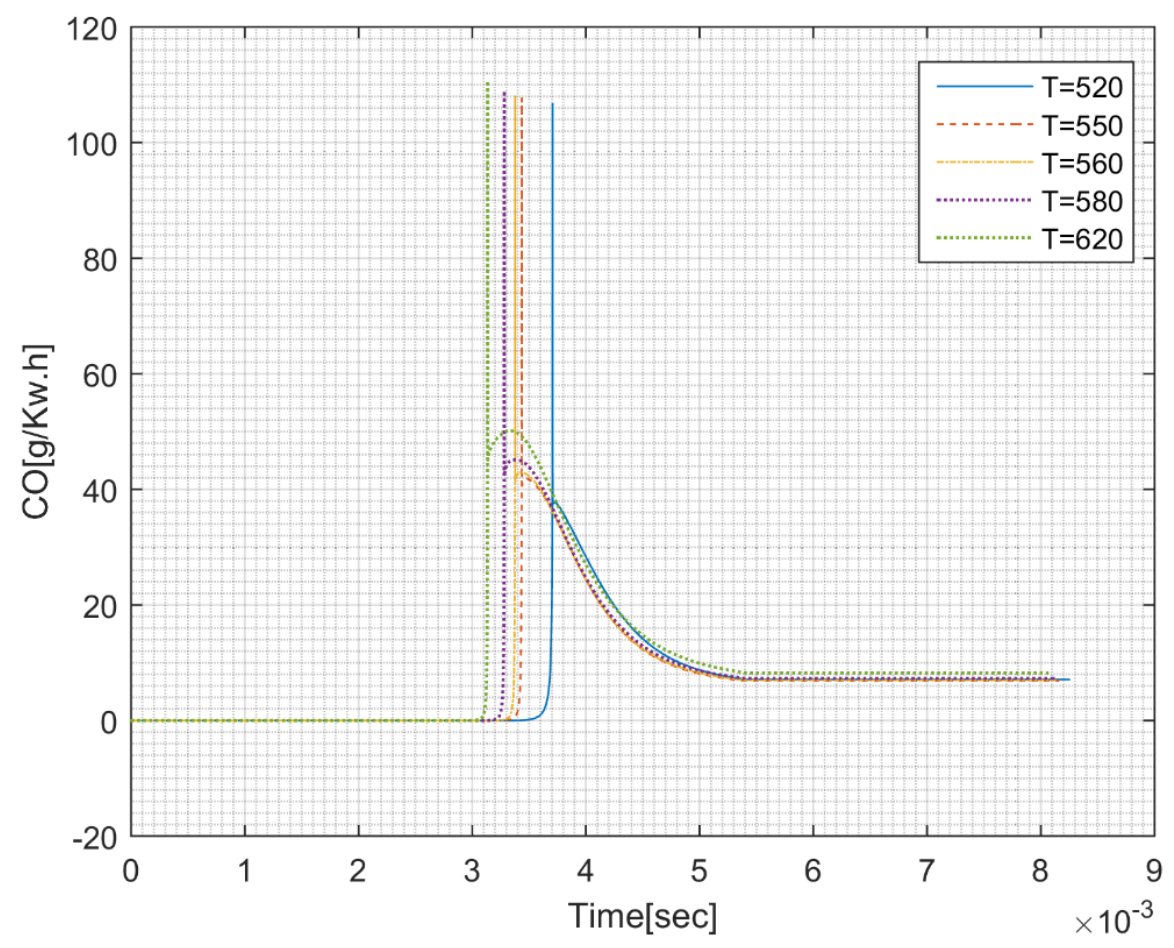

Figure $57 \mathrm{CO}$ emissions at different intake temperature.

\subsection{Equivalence ratio}

\subsubsection{Equivalence ratio review}

The fuel to oxidant ratio is among the basic parameters, which are used to evaluate the engine performance. The equivalence ratio refers to the ratio between the actual fuel-air ratio and the stoichiometric one as in equation 6.1 [136].

$$
\phi=\frac{(\text { fuel/air })_{\text {actual }}}{(\text { fuel/air) })_{\text {stoichiometric }}}
$$

When $\phi$ is equal to one, this means that the process is stoichiometric (no excess fuel or air). However, if $\phi>1$, this refers to a rich mixture combustion (excess fuel or less air than the stoichiometric state). In the other side, if $\phi<1$, this indicates that the mixture is lean (excess air or less fuel than stoichiometric state) [136].

The nature of the fuel-air mixture, which is defined by the equivalence ratio as rich or lean mixture affects the system performance significantly. For example, lean mixtures have many positive features like the higher thermal efficiency and less engine knock. In addition, low emissions levels like CO an UHC are obtained at lean combustion conditions while ultra-lean mixtures result with low $\mathrm{NO}_{\mathrm{x}}$ emissions. However, those mixtures have low reaction rates, misfiring events, operation instabilities, low heat release rate, and sensitivity to mixing process [92]. In the other side, burning process in rich mixture is similar to the stoichiometric one. It has less misfiring events and less instabilities but with more emissions levels especially $\mathrm{CO}$ and $\mathrm{UHC}$, in addition to low efficiencies in comparison with lean mixtures [92].

In this chapter, the effect of the equivalence ratio and the intake temperature are illustrated. Variable equivalence ratios will be obtained by changing the fuel percentage above or below the stoichiometric value. Different intake temperatures have been examined as well to obtain the dual effect caused by both 
of the equivalence ratio and the intake charge temperature. The initial conditions for each equivalence ratio are presented in Table 6.

Table 6 Initial condition for different equivalence ratios

\begin{tabular}{cccccccccccc}
\hline \multicolumn{1}{c}{ Fuel } & \multicolumn{10}{c}{ Air } \\
\hline $\boldsymbol{\phi}$ & $\mathrm{CH} 4 \%$ & $\mathrm{C} 2 \mathrm{H} 6 \%$ & $\mathrm{C} 3 \mathrm{H} 8 \%$ & $\begin{array}{c}\text { Air to } \\
\text { fuel }\end{array}$ & $\begin{array}{c}\text { Air-fuel } \\
\text { mass }(\mathrm{g})\end{array}$ & $\mathrm{N2 \%}$ & $\mathrm{O} 2 \%$ & $\begin{array}{c}\mathrm{R} \\
(\mathrm{kJ} / \mathrm{kg} . \mathrm{K})\end{array}$ & $\mathrm{T}_{0}(\mathrm{~K})$ & $\mathrm{P}_{0}(\mathrm{Kpa})$ \\
\hline $\mathbf{0 . 8}$ & 80 & 11 & 9 & 21.27 & 0.01766 & 76.7 & 23.3 & 0.2965 & 520 & 101 \\
\hline $\mathbf{0 . 9}$ & 80 & 11 & 9 & 18.86 & 0.01760 & 76.7 & 23.3 & 0.2975 & 520 & 101 \\
\hline $\mathbf{1 . 0}$ & 80 & 11 & 9 & 16.95 & 0.01754 & 76.7 & 23.3 & 0.2985 & 520 & 101 \\
\hline $\mathbf{1 . 1}$ & 80 & 11 & 9 & 15.38 & 0.01749 & 76.7 & 23.3 & 0.2991 & 520 & 101 \\
\hline $\mathbf{1 . 2}$ & 80 & 11 & 9 & 14.08 & 0.01743 & 76.7 & 23.3 & 0.3004 & 520 & 101 \\
\hline
\end{tabular}

6.2.2 Variable equivalence ratio at constant intake temperature:

The free-piston velocity at different positions is illustrated in Figure 58; the figure was zoomed near the TDC to show the effect of equivalence ratio in shape of the profile at this area. The semi-egg shape is clear for all of the equivalence ratio values. The piston speed starts to decrease near the TDC due the increasing in the in-cylinder pressure. During the combustion process, the piston moves in the opposite direction by the force of the combustion pressure. The location of the ignition location shifts away from the cylinder head as the equivalence ratio increased. However, when the equivalence ratio is above the stoichiometric value $(\phi=1)$, the TDC will shift toward the top of the cylinder. In general, Figure 58 demonstrates the sensitivity of the piston dynamic to the thermodynamic process. The variations in the TDC location are the monitor of this sensitivity. More details are discussed in this chapter to identify the reasons behind such sensitivities in the performance. 


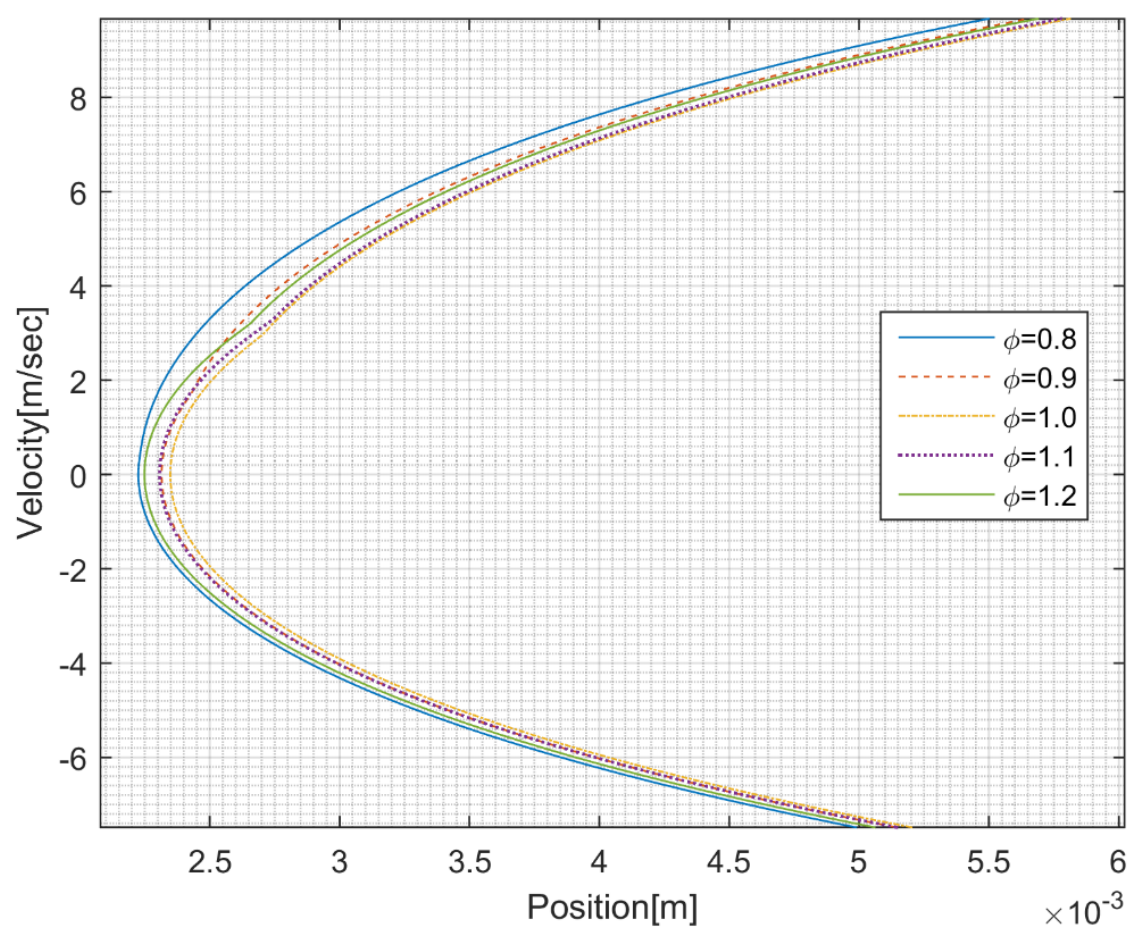

Figure 58 Velocity-position diagram.

Figure 59 describes the heat release rate produced by a fuel-air mixtures burning at different equivalence ratios. Considering the ignition time, the figure indicates that, lean mixtures $(\phi<1)$ ignite earlier than rich mixtures by $0.25 \mathrm{~ms}$, this early combustion event can be explained by the higher reactions rate of the species. The excess $\mathrm{O}_{2}$ concentrations will speed the destruction rate of the fuel molecules since $\mathrm{O} 2$ molecules have less heat capacity than the fuel molecules. Another reason belongs to the relatively higher compression ratios. The increasing in-cylinder pressure caused by the higher compression ratios will assist the fuel-air mixture to reach the self-ignition ignition point faster. This fact is obvious in Figure 58. TDC locations indicate that higher compression ratios are achieved at lean conditions. Another observation is the amount of HRR within the applied range of equivalence ratio. The relation between the equivalence ratio and the HHR value is found to be complicated. This fact has many considerations that belong to the fuel density, species reactions rate and ignition time and duration. All of those factors can affect the HRR value since fuel burning process is defined by them. In the other side, rich mixtures is more difficult to be evaluated since partial combustion process can happen due to the lack in $\mathrm{O}_{2}$ molecules. In general, near stoichiometric process will show the highest HRR and as the process goes toward the rich or the lean state, the value of the HRR will have some drop. 


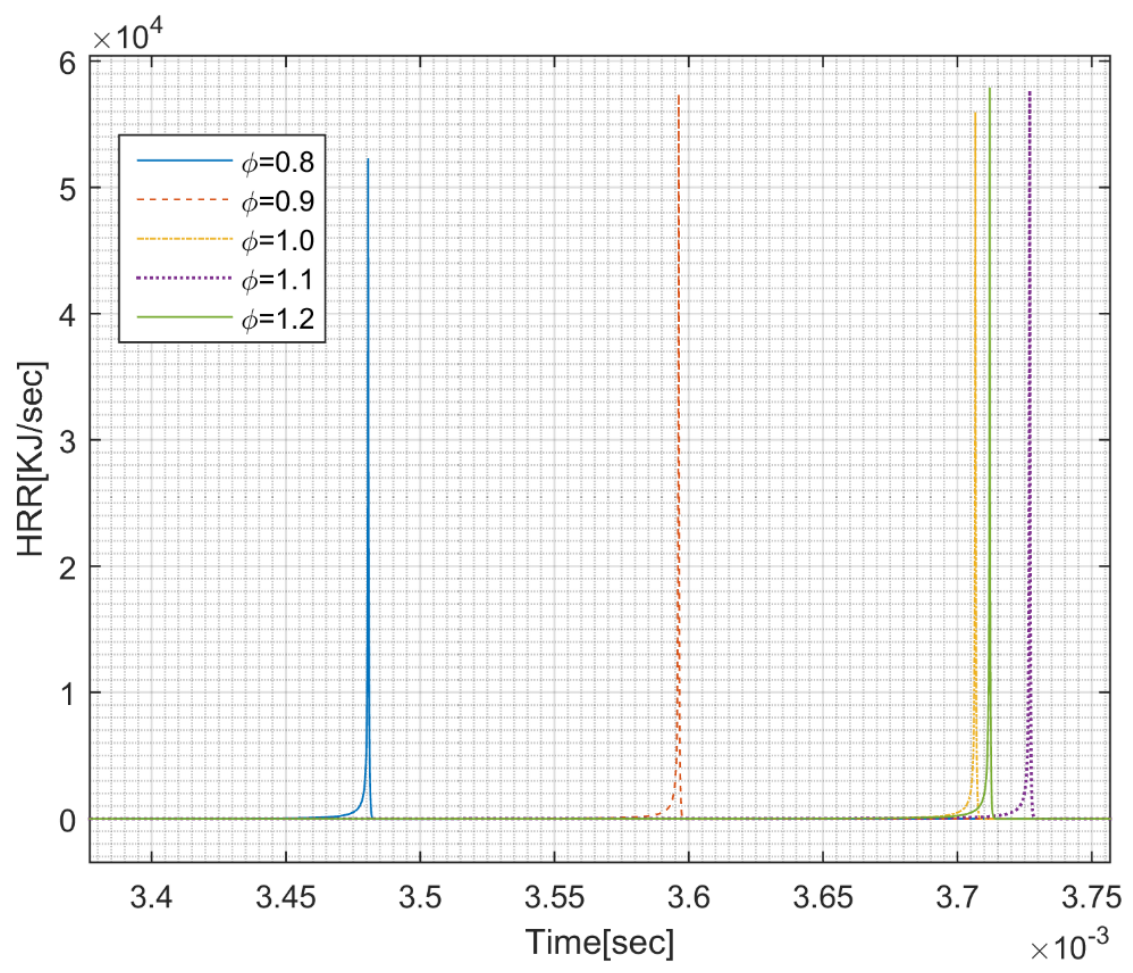

Figure 59 HRR vs. equivalence ratio .

The combustion process at variable equivalence ratio was presented in terms of the in-cylinder pressure as in Figure 60. The ignition in the case of the lean mixtures is earlier than the one in the rich mixtures. In addition, the self-ignition pressure is higher for lean mixtures than the rich mixtures, which indicates that earlier ignition events are not controlled by the self-ignition pressure or temperature. Basically, the compression process of the fuel-air mixture is not the same for all type of mixtures. For low equivalence ratio mixtures, the mixture will undergo high pressures during the compression stroke in the time before the combustion. The ignition in such cases will be at the TDC or before, so early combustion events can occur. In the other side, the rich mixtures have different compression process; they will ignite lately after the TDC due to the less applied compression force, as it is clear in Figure 60. The difference in the ignition time and the self-ignition conditions will result with system performance variations. For example, the alternator work output will be affected by the pressure variations. This show how the piston dynamics are directly proportional to the in-cylinder process in the free-piston devices. The peak pressure reflects another effect of the equivalence ratio. High peak pressures are coupled with low equivalence ratios and vice versa. The ignition time and location are the main factors in defining the peak pressure value. In the case of the lean mixtures, the pressure increases in the same direction of the piston (compression stroke) as the ignition time is earlier at the TDC. In contrast for rich mixtures, the pressure increases in opposite direction to the piston motion (expansion stroke) as late ignition events are occurred. In general, this behavior in the in-cylinder pressure explains the reason behind preferring lean mixtures in $\mathrm{HCCl}$ combustion. In $\mathrm{HCCl}$ devices, the ignition process is very rapid and has no flame front like the one presented in the rich mixture combustion. 


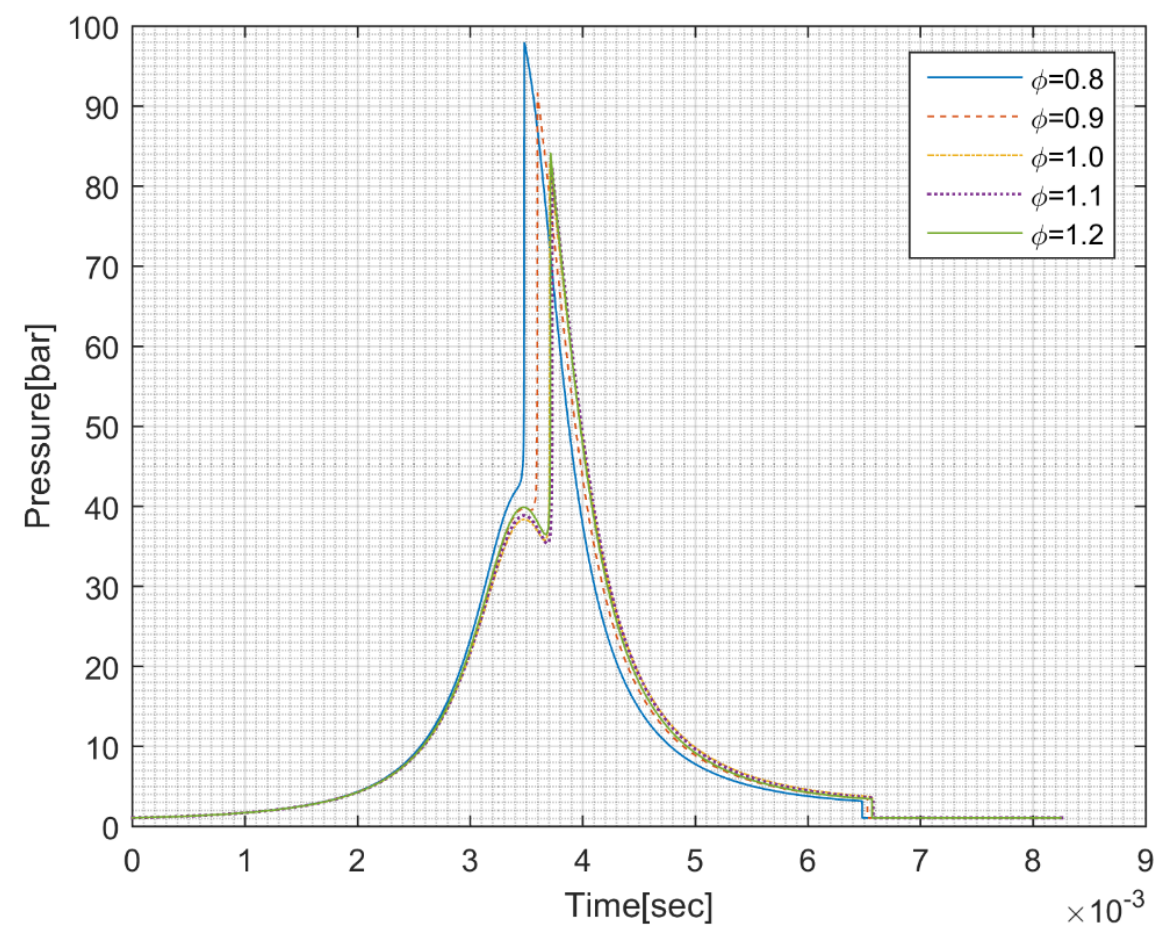

Figure 60 In-cylinder pressure at different equivalence ratios.

Figure 61 demonstrates the compression ratio at different equivalence ratio values. Lean and rich mixtures share similar compression ratio values where they have higher compression ratio than this found stoichiometric conditions. The nature of the fuel-air mixture burning explains these variations in the compression ratio. In the lean mixtures case, the excess air content will absorb some of the compression heat, which will make the charge as a heat sink due to the low heat capacity ratio compared to the stoichiometric and rich conditions. This will increase the mixture temperature and therefore the incylinder pressure. At the same time, the piston will move to points that are closer to the cylinder head, which will result with higher compression ratio. In the case of the rich combustion, the increase in the overall pressure and temperature will be slower due to the higher heat capacity ratios; therefore, the piston stays longer in the compression stroke and the combustion can happen later after the TDC. This will require that the piston will reach the maximum possible point near the cylinder head (high CR). At stoichiometric combustion, the process will be in neutral state between the lean and rich combustion. From chemical perception, the species reactions rate is the dominate factor that defines the end of the compression stroke and the start of the ignition. Lean mixtures have higher oxidation rate due to their high $\mathrm{O}_{2}$ concentrations. Based on that, the ignition process will be enhanced and it will start earlier as an efficient heat releasing will be obtained. In the other side, rich mixtures have less $\mathrm{O}_{2}$ content, this results with a slower reactions rate and incomplete burning of the high concentrated fuel mixture, therefore higher compression ratio are observed. 


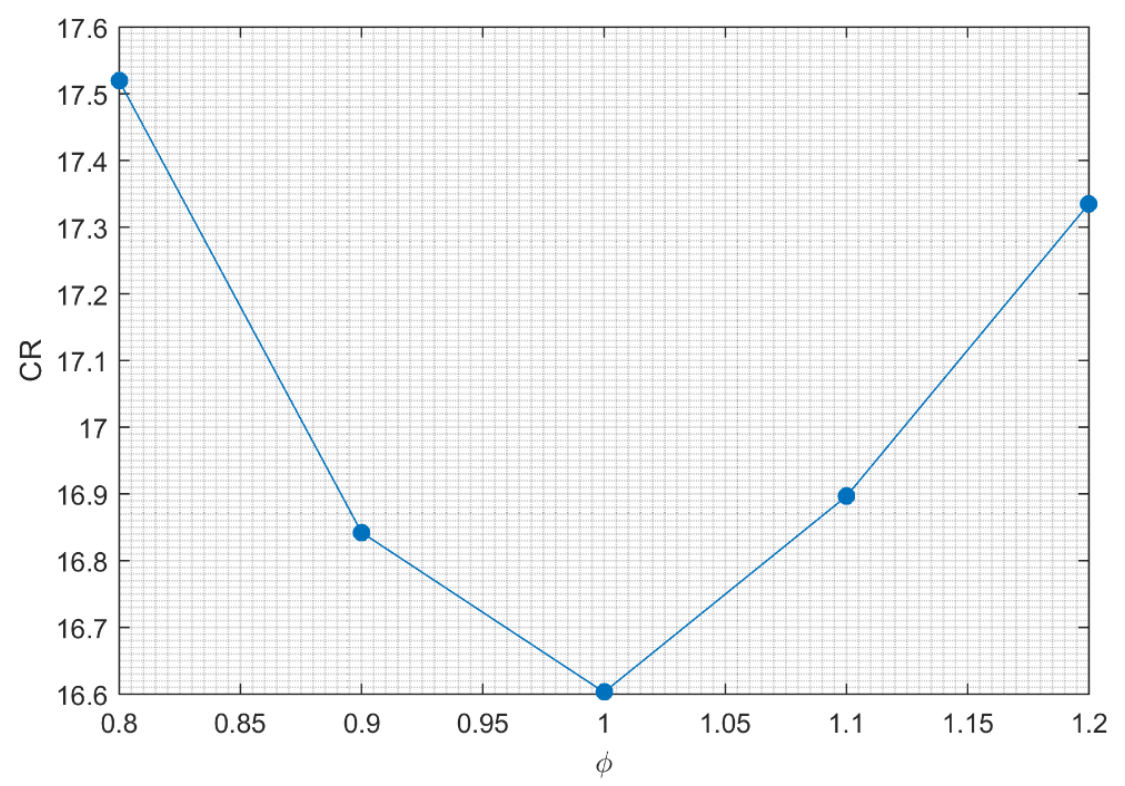

Figure 61 Compression ratio vs. equivalence ratio.

In Figure 62, the in-cylinder pressure is demonstrated as a function of the piston position at different equivalence ratios. The gas mixture, compression, combustion and the expansion present the main cycle stages. The difference in the ignition location is clear for each equivalence ratio. The TDC location is following the compression ratio diagram. It becomes farther from the cylinder head when the equivalence ratio is near the stoichiometric value, while it is closer to the cylinder head for both of the rich and lean mixtures. The ignition location is presented clearly as well, it is almost at the TCD point in the case of lean mixture; however, in the case of the rich mixture, the ignition occurs after the TDC. The $\mathrm{HCCl}$ combustion process is presented in the case of the low equivalence more sharply by appearing as a rapid ignition event. In the other side, the combustion of the high equivalence ratio mixtures is slower with some gradual burning zone especially at the beginning of the combustion process.

Figure 63 consider the variations in the temperature as a function of the piston positon for different equivalence ratios. The combustion process and the expansion stroke represent the difference between each case while the compression stroke is similar for all cases. The ignition time is responsible about the variation in the combustion process. The TDC becomes closer to the cylinder head as the mixture becomes leaner while the peak temperature is decreasing slightly for lean mixtures due to the $\mathrm{O} 2$ dilution effect in cooling the whole charge. 


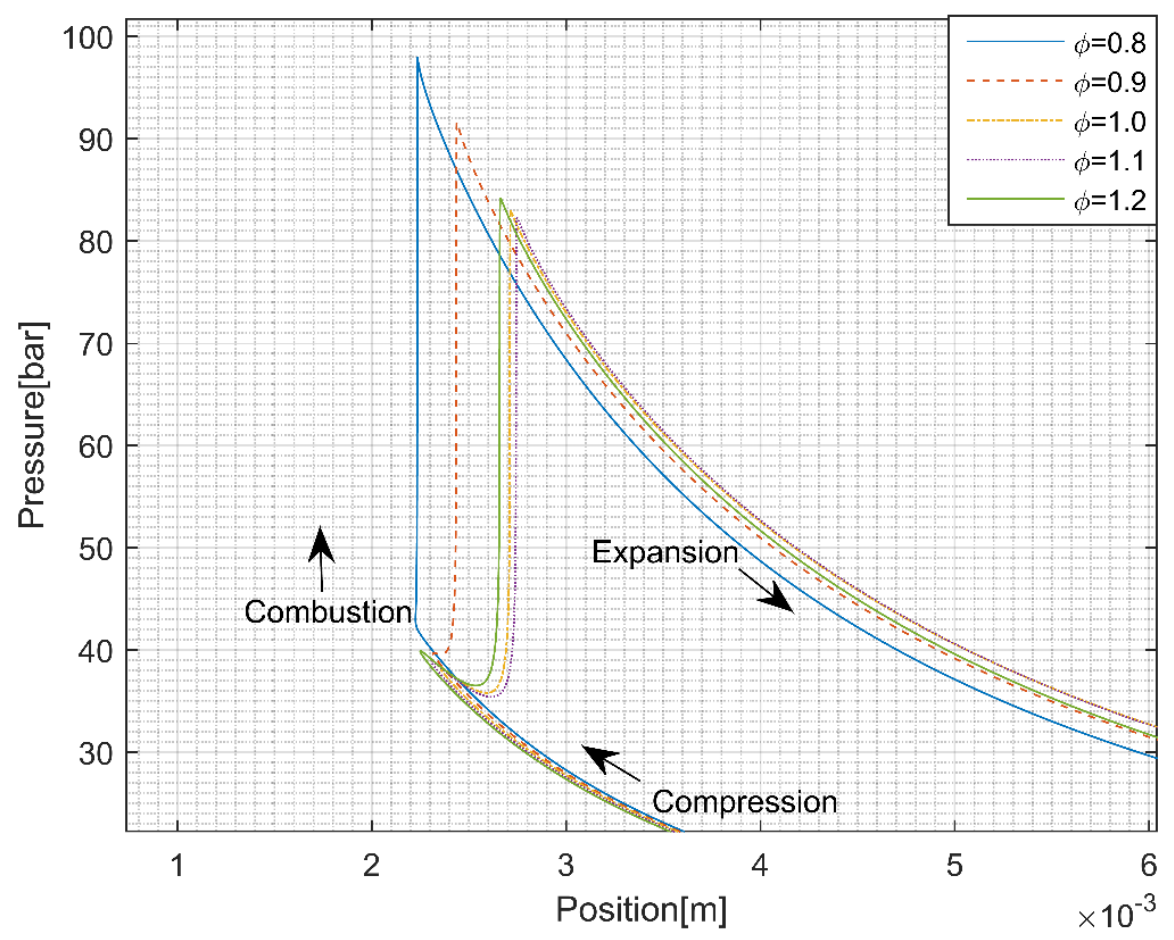

Figure 62 in-cylinder pressure-position diagram.

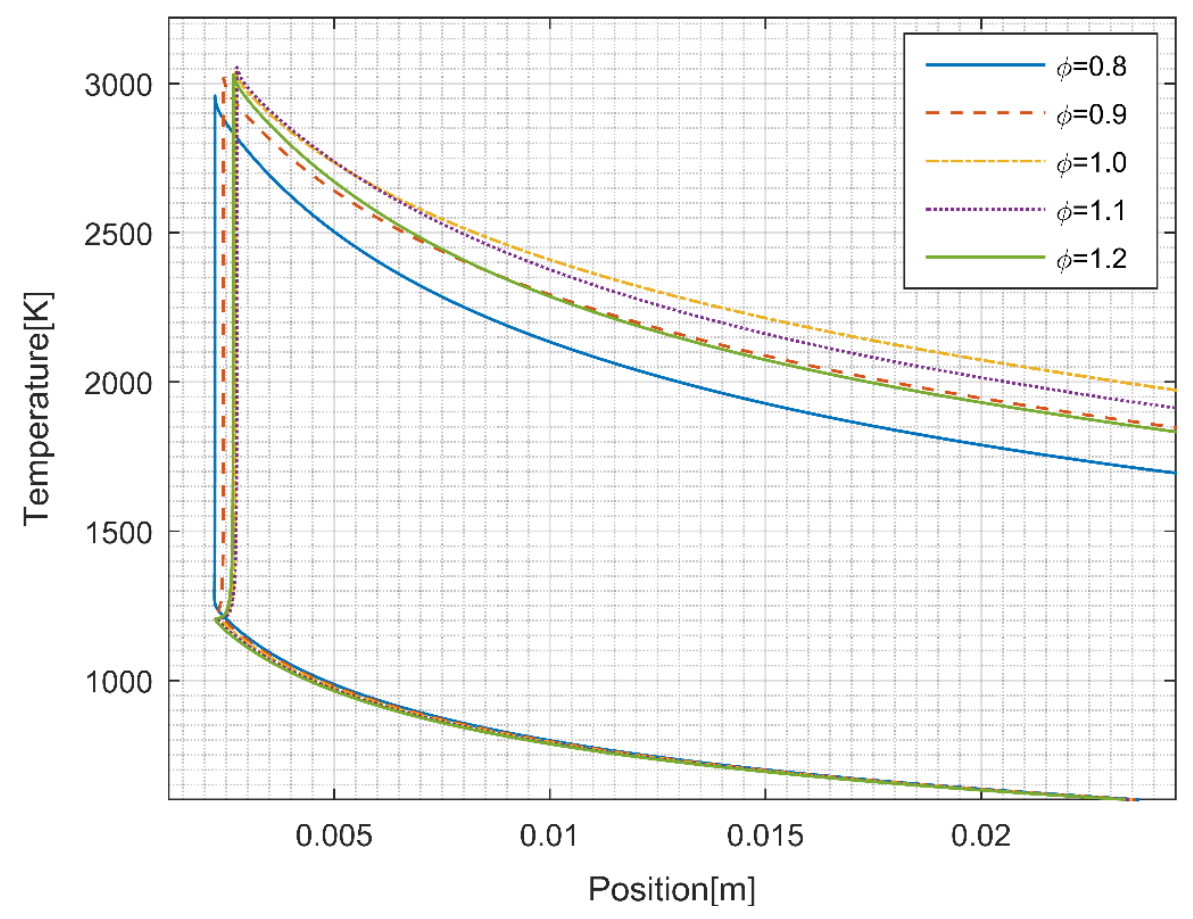

Figure 63 in-cylinder temperature-position diagram. 
In Figure 64, the in-cylinder temperature is presented at different equivalence ratios. The figure was zoomed near the combustion area to show the effect on this stage. By comparing the self-ignition temperature for the different equivalence ratios, it is found that lean mixtures have higher ignition temperatures than those in the rich mixtures. The low heat capacity of the air compared to the fuel will allow the mixture to reach higher temperatures before the ignition. In addition, the nature of the reactions rate is much faster in the case of the lean mixtures, which requires higher temperatures to assist such reactions rate. In the other side, the burning process of the rich mixtures is slower due to the insufficient $\mathrm{O}_{2}$ concentration. At the same time, the heat capacity is much higher for those mixtures due to the higher fuel intensity. Those factors will reduce the self-ignition temperature dramatically. By considering the ignition delay, this time is greater for rich mixtures since they have more resistance to be ignited than the lean mixtures, this make the last, more preferable in $\mathrm{HCCl}$ combustion devices.

Referring to the peak temperature, Figure 64 shows that this temperature is increasing as the equivalence ratio increases where a greater HRR is obtained at high equivalence ratios. In addition, the excess air will reduce the combustion temperature by diluting the fuel content within the combustion chamber, this will reduce the possibility of having extreme hot zones inside the cylinder. From emissions perception, in the case of the stoichiometric combustion $(\phi=1)$, the ignition happens after the TDC, but the combustion will be complete so no $\mathrm{CO}$ or UHC will be produced. The same trend is presented in the lean mixtures since there are plenty of air to oxidize the fuel. However, rich mixture will have incomplete combustion events so more $\mathrm{CO}$ and $\mathrm{UHC}$ are expected. $\mathrm{NO}_{x}$ emissions are more difficult to be evaluated at this point, the maximum peak temperature is an indicator for those emission levels, but the illustrated difference in the peak temperature is not very high to decide based on it. In addition, the nature of the $\mathrm{NO}_{\mathrm{x}}$ emissions formation requires sufficient $\mathrm{O}_{2}$ molecules that are presented excessively in the lean mixture. 


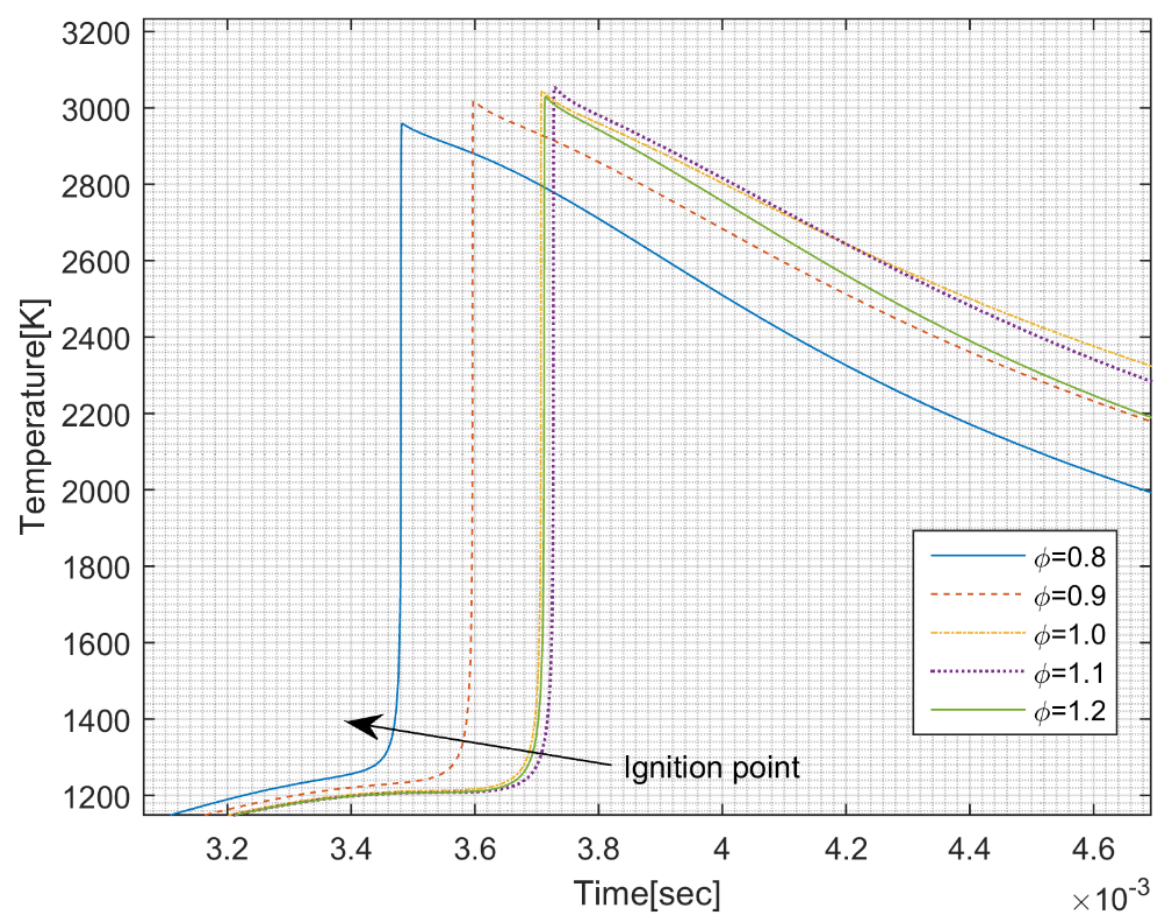

Figure 64 In-cylinder temperature at different equivalence ratios.

The effect of the equivalence ratio on the work output ratio is described in Figure 65. A noticeable drop in the work output ratio is observed as the equivalence ratio increased from the lean sate to stoichiometric one. Such drop refers potentially to the delay in the ignition and to the higher self-ignition pressure as discussed before. Beyond the stoichiometric conditions, the work output ratio will start to increase slightly due to the increase in the HRR of the combustion process. Within the applied range of the equivalence ratio, the lean mixtures are the most efficient. The reduction in both of the HRR and the resulted combustion pressure are among the reasons that can cause such potential changes in the alternator work output. 


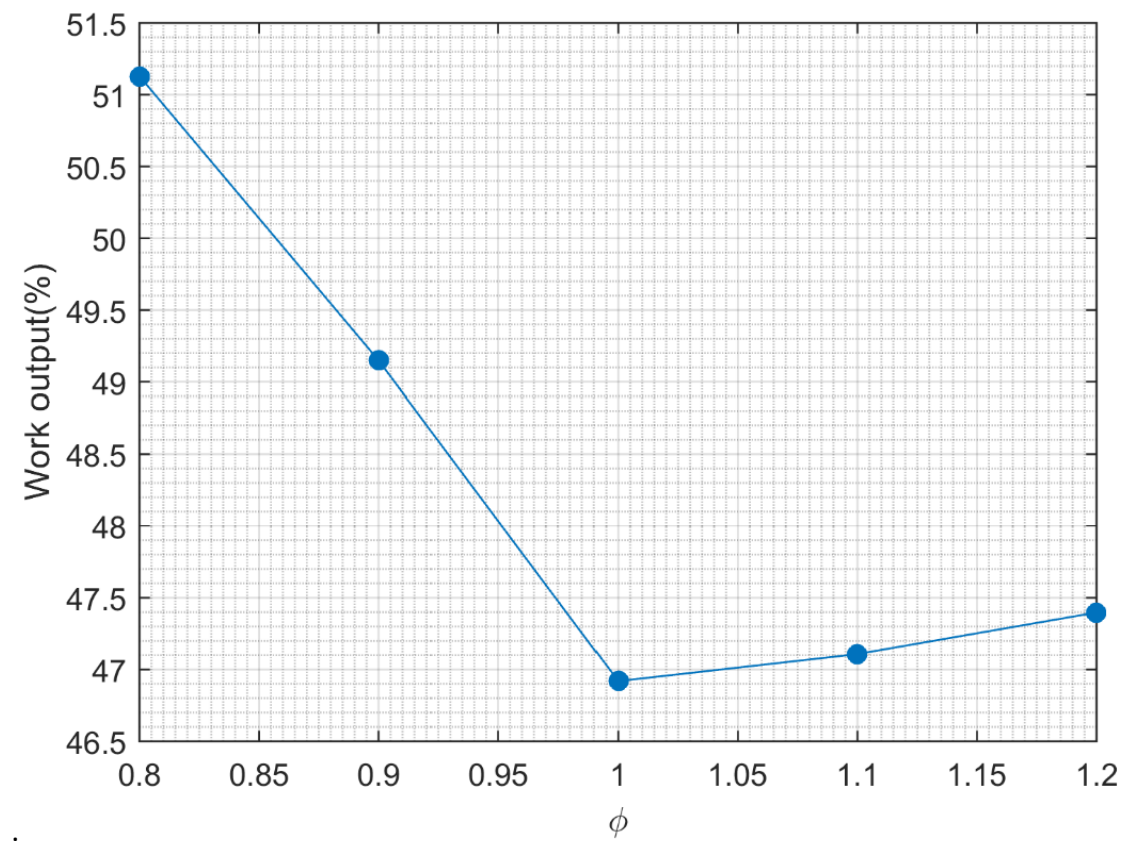

Figure 65 Work output vs. equivalence ratio.

$\mathrm{NO}_{x}$ formation is shown in Figure 66, the formation rate of those emissions start at the combustion process instantaneously. The formation rate is increasing rapidly at this stage due to the plenty of fuel and air concentrations. By the end of the burning process, the formation rate will start to decrease in a gradual manner. The $\mathrm{NO}_{x}$ formation becomes constant in the remaining time of the cycle since an equilibrium state will be reached. By reviewing the $\mathrm{NO}_{\mathrm{x}}$ emissions level, it is relatively high for lean mixtures compared to those levels when rich or stoichiometric conditions are applied. Basically, this refers to the noticeable difference in the $\mathrm{O}_{2}$ content between the lean and rich mixtures. As explained in the previous chapter, the $\mathrm{O}_{2}$ molecules have less heat capacity than this of the fuel molecules; this difference in the heat capacity will affect the reactions rate of the $\mathrm{NO}_{\mathrm{x}}$ emissions by increasing the oxidation rate of the $\mathrm{N}_{2}$ molecules. In addition, the plenty of the $\mathrm{O}_{2}$ will speed up the fuel oxidation process while the excess air in the intake charge will give the chance to the $\mathrm{N}_{2}$ molecules to be converted to the $\mathrm{NO}_{x}$ form. However, the reactions rate in the case of the rich mixtures is much slower due to the higher heat capacity for the involved molecules and the less available $\mathrm{O}_{2}$ concentrations. It is expected for the ultra-lean mixtures to have less $\mathrm{NO}_{\mathrm{x}}$ emissions due to the lack in the released heat. The insufficient fuel molecules in the intake charge will have low HRR; this fact will limit the $\mathrm{N}_{2}$ and $\mathrm{O}_{2}$ reaction process. 


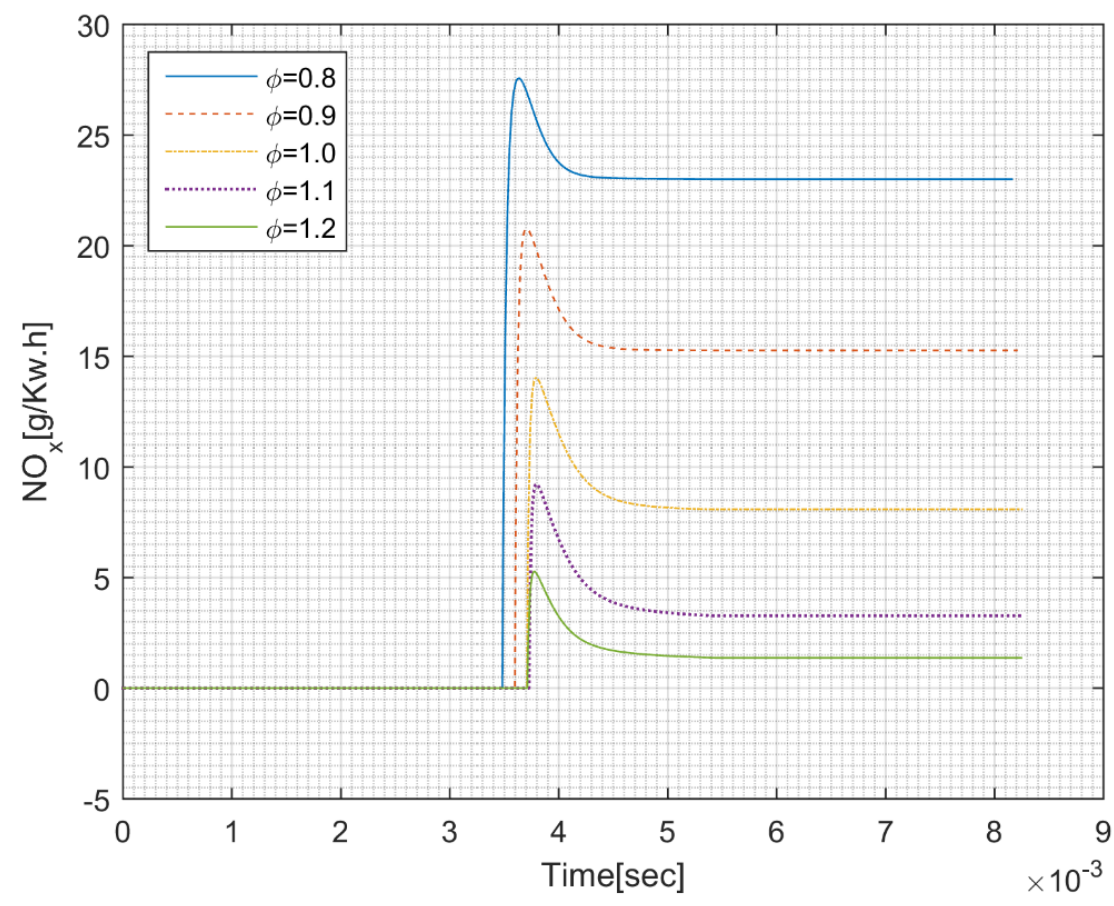

Figure $66 \mathrm{NO}_{\mathrm{x}}$ emissions at different equivalence ratios.

Other emissions like $\mathrm{CO}$ and $\mathrm{UHC}$ are presented in the free-piston cycle products, the variations in the equivalence ratio has a direct relation to these emissions as shown in Figure 67 . For example, lean mixtures have the lowest CO outcomes while stoichiometric mixtures $(\phi=1)$ have moderate CO levels. Finally, rich mixtures have the highest $\mathrm{CO}$ emissions formation rate since these mixtures have the lowest $\mathrm{O}_{2}$ contents. In addition, Figure 67 shows that the changes in the $\mathrm{CO}$ formation vary dramatically within the entire applied rich mixtures. However, lean mixtures are behaving in similar manner even for any equivalence ratio that is greater than one. The similarity refers to the fact that those mixtures have plenty of $\mathrm{O}_{2}$ molecules, which will assist the complete burning of the low concentrations fuel mixture but with longer periods.

High CO levels are usually coupled with high UHC levels since both of those emissions are caused by of the incomplete burning of the fuel. Based on that, it is expected to have higher UHC levels within the rich mixtures combustion due to the highly concentrated fuel molecules. Incomplete combustion events have a significant effect on the work output ratio of the engine, this makes the rich mixtures inefficient compare to the lean ones, which often have higher work output ratio.

The quality of the fuel-air combustion can be measured also by predicting $\mathrm{CO}_{2}$ levels. An interested observation can be noticed in Figure 68 where $\mathrm{CO}_{2}$ emissions formation is presented at different equivalence ratios. Figure 68 indicates that $\mathrm{CO}_{2}$ formation increase as the equivalence ratio decreases. This behavior is expected since better burning events are achieved at higher $\mathrm{O}_{2}$ concentrations. However, when the mixture starts to be in the lean zone, the rate of the $\mathrm{CO}_{2}$ formation will start to be constant. This is observed because the fuel will be completely burned to form $\mathrm{CO}_{2}$ molecules with no chance for $\mathrm{CO}$ molecules. Such conclusions proof that ultra-lean combustion is among the most suitable environments 
for the $\mathrm{HCCl}$ engines. The higher work output ratios and low emissions are the most desirable features of $\mathrm{HCCl}$ environments.

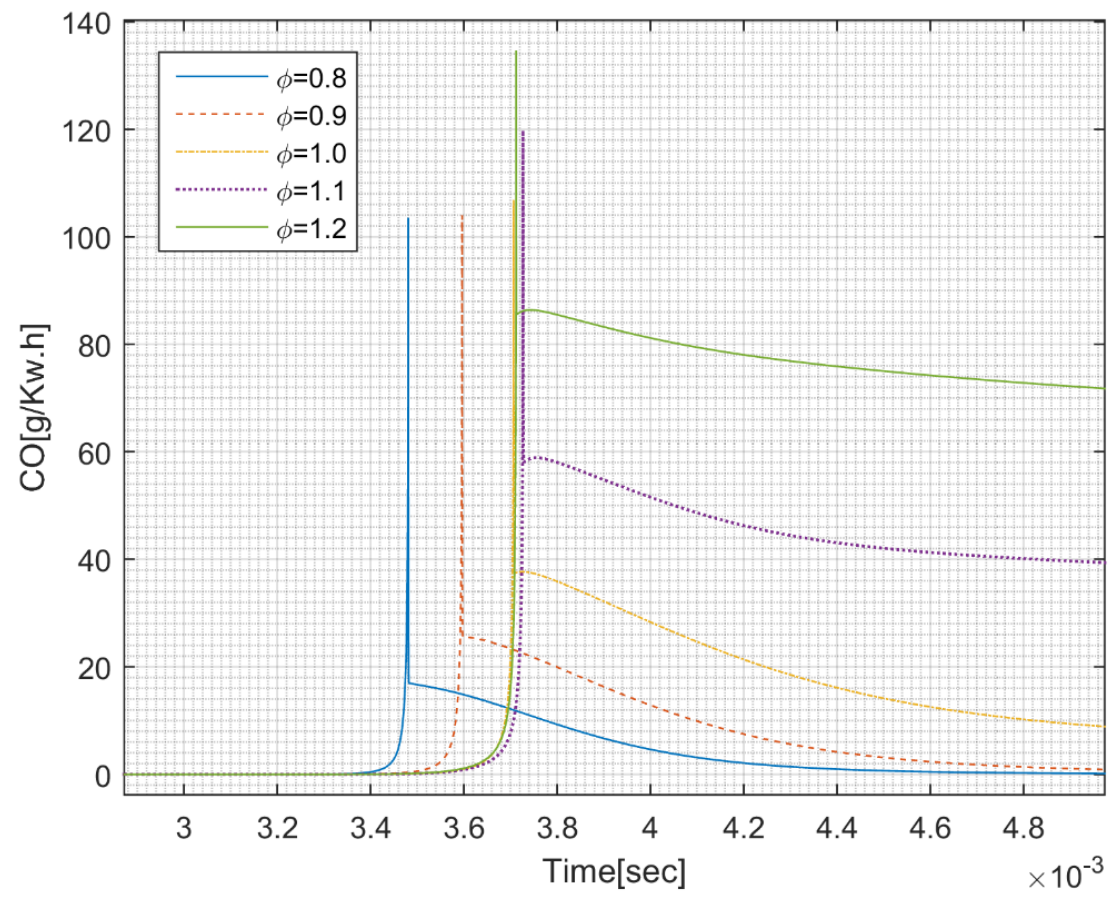

Figure $67 \mathrm{CO}$ emissions at different equivalence ratios.

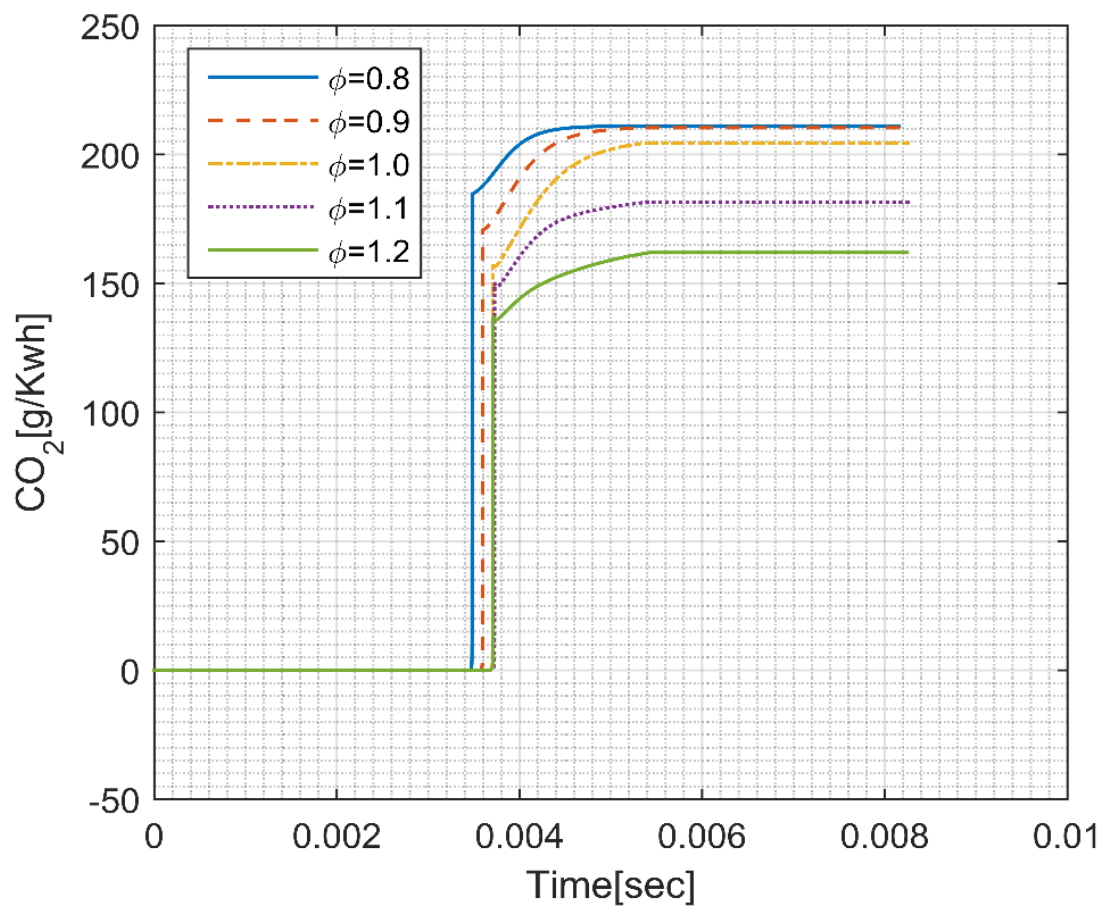

Figure $68 \mathrm{CO}_{2}$ emissions at different equivalence ratios. 


\subsubsection{Equivalence ratio effect at variable intake temperature.}

The intake gas temperature is one of the effective factors that can affect the overall combustion process. The variations in this temperature can be ether in purpose like in the case of cooled and hot EGR, or it can be due the improper gas exchange process at the intake event. In this section, the equivalence ratio effect at different intake temperatures has been investigated.

In Figure 69, the peak in-cylinder pressure is presented at different intake temperatures and equivalence ratios. The intake pressure and mass have been held constant during the simulations. At low intake temperature values, Figure 69 shows that, the lean mixtures have the maximum peak pressures, which refers to many reasons like the time and location of the ignition. However, at relatively higher intake temperatures, the effect of the equivalence ratio will start to be weak. In the case of the rich mixtures, the increasing in the intake temperature from low to moderate values will rise the peak pressure significantly within this temperature range. A slight reduction in the peak temperature is observed as the intake temperature exceeded the moderate values $\left(T_{\text {in }}>560\right)$. In the other side, the stoichiometric conditions have the minimum peak pressure at all intake temperatures levels. This is referred to the low compressions ratio compared to those at lean or rich combustion. In general, increasing the intake temperature will reduce the peak pressure in the case of the lean mixtures. In the other side, it will increase it if stoichiometric or rich condition are applied. Such conclusion stands behind the fact that, the high intake temperatures include early ignition events. From chemical perception, the mixture temperature affects the reaction rate by advancing the destruction and construction of the different species. This will speed the required reactions of the ignition and therefore earlier combustion events will be observed. Based on that, the ignition location will be before the TDC in the case of the lean mixture and near the TDC in the case of the rich mixtures. In fact, the absence of the fixed TDC location in the freepiston engine reflects the real effect of every parameter within the engine environment.

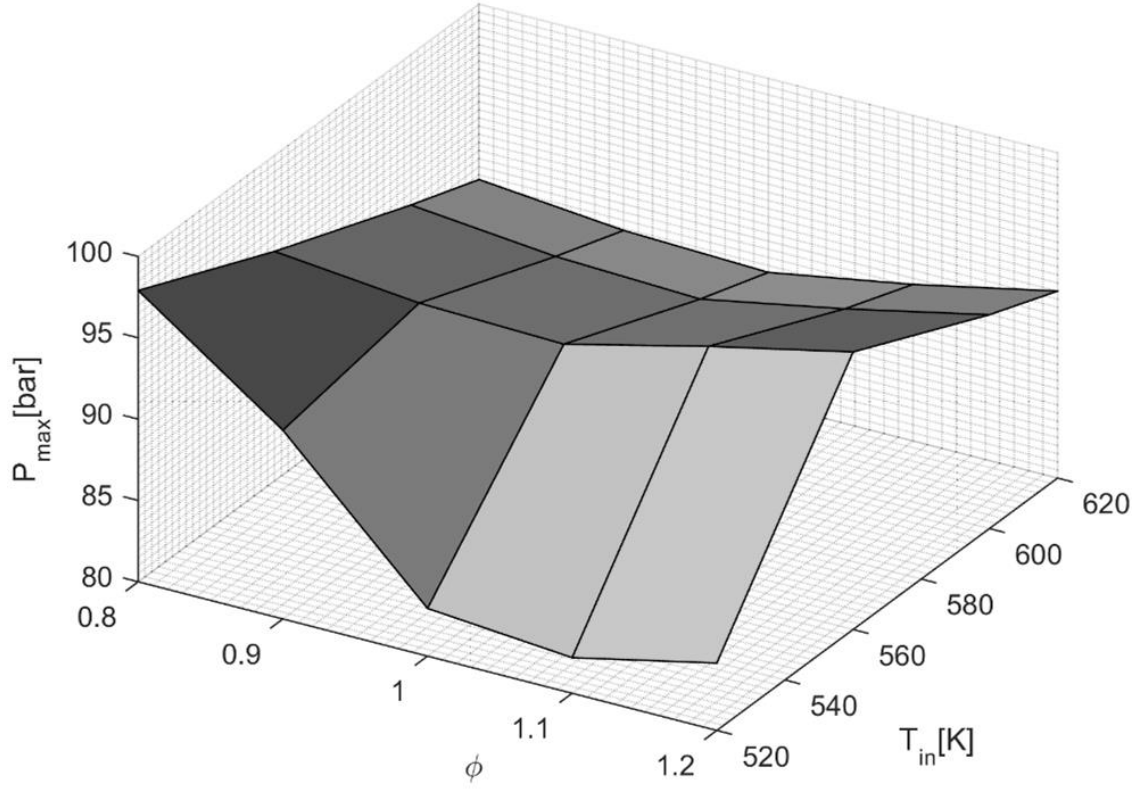

Figure 69 Peak pressure at different intake temperatures. 
The compression ratio is also an important engine parameter since it has a direct relation to the stability of the performance. In the last section, the effect of the equivalence ratio on the compression ratio had been discussed. The results showed that, the stoichiometric conditions had the minimum compression ratio while this ratio started to increase as the mixture became rich or lean. The dilution effect of the excess air found in the lean mixtures is the responsible about increasing the compression ratio. In the other side, the late ignition in the case of the rich mixtures explains the reason behind their high compression ratio as well. The effect of the equivalence ratio at different intake temperatures is explained in Figure 70, which indicates that rich and lean mixtures combustion is attached with the highest compression ratios at all intake temperature levels. However, the stoichiometric conditions have the lowest compression ratio values. At the same time, for any equivalence ratio, the compression ratio increases linearly as the intake temperature increases. Higher intake temperatures Advance the ignition time and the ignition location by speeding up the species reactions rates so higher in-cylinder pressure will be observed.

As the in-cylinder pressure is the motivator of the piston dynamics, a variation in the work output ratio of the alternator is expected. This fact is illustrated in Figure 71 where the alternator work output is demonstrated at different intake temperatures and equivalence ratios. The results show that, lean and relatively cold mixtures have the optimal work output ratio. Typically, this refer to the high in-cylinder pressures, which are achieved at those conditions. In addition, high temperature mixtures indicate less work outcomes than those with low temperatures. The increasing in the intake temperature affects all types of mixtures, but lean combustion is the most affected since a sharp reduction in the work output ratio is predicted at high intake temperatures. In contrast, rich and stoichiometric charges are less affected where a shallow decrease in the work output ratio is observed.

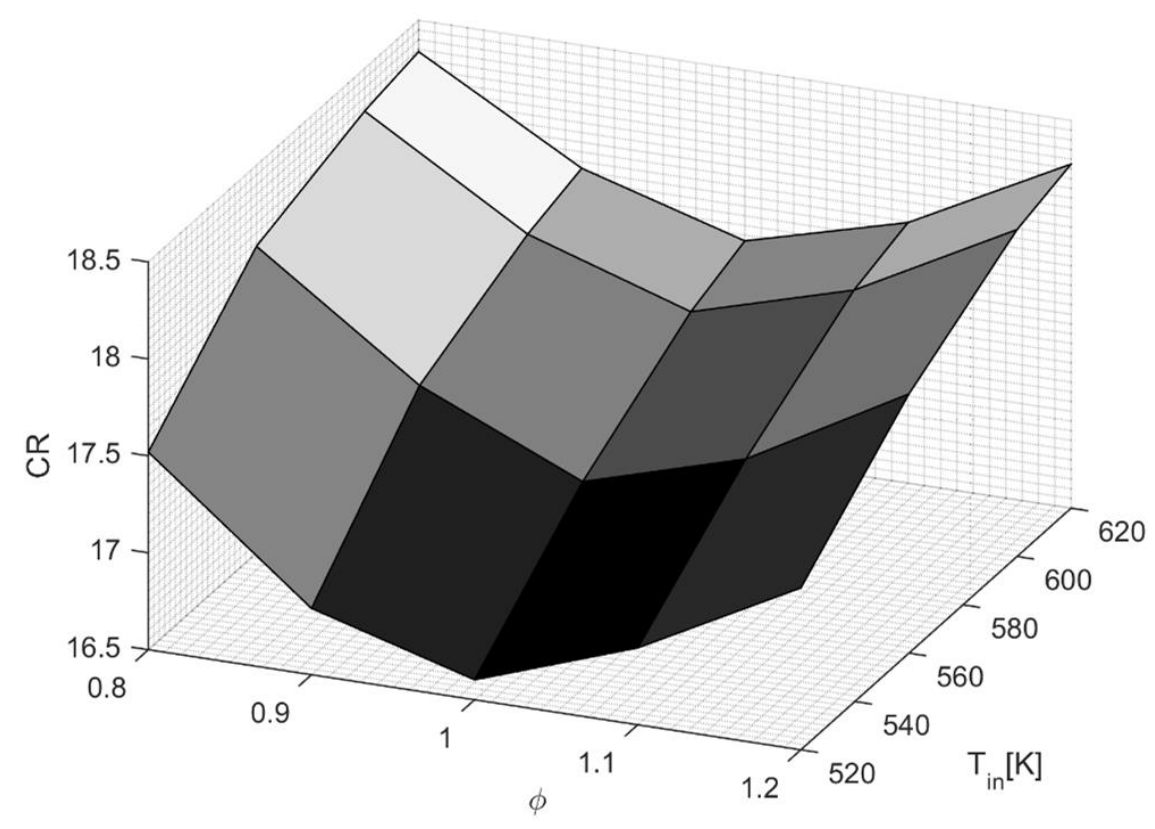

Figure 70 Compression ratio at different intake temperatures. 


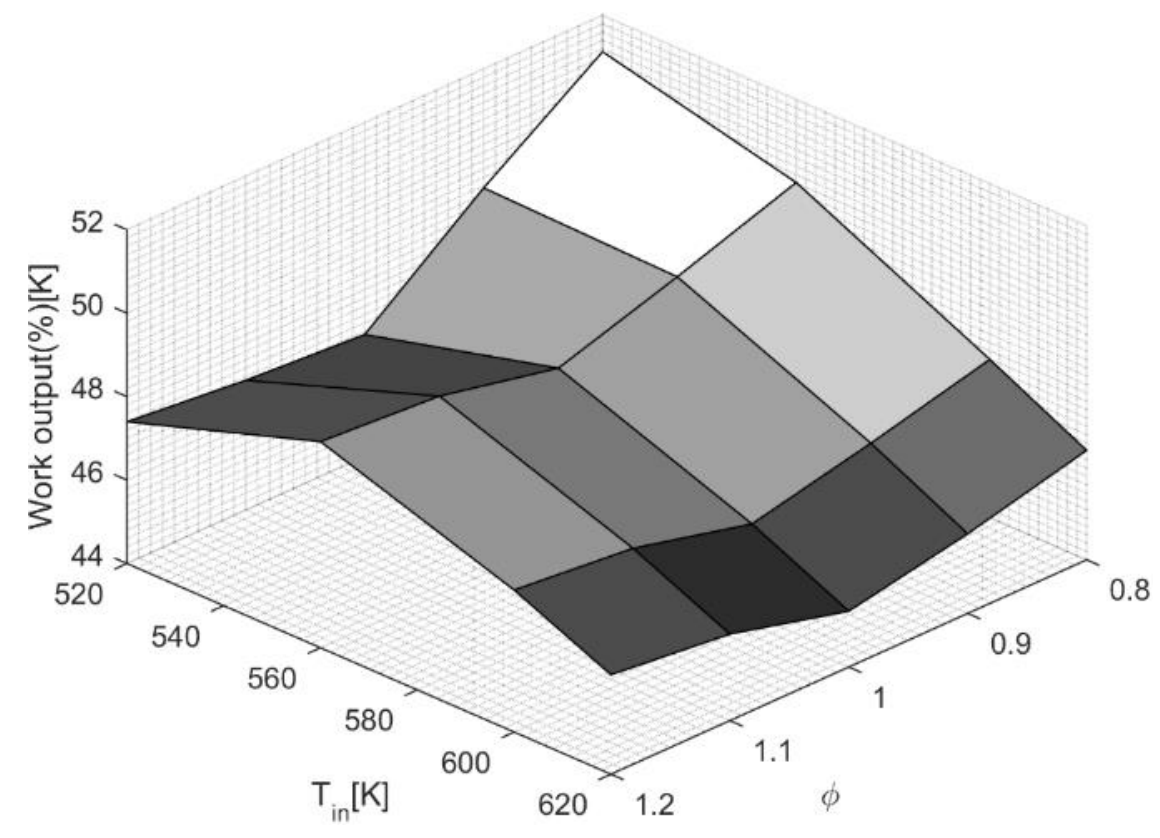

Figure 71 Work output ratio at different intake temperatures.

The effect of the equivalence ratio on the in-cylinder temperature had been investigated previously. It was noticed that, the peak temperature is high in the case of the rich and stoichiometric conditions while it is less in the case of the lean mixtures. The reason for such behavior is the higher HRR, which presents the burning of air-fuel mixture. Mixtures with high equivalence ratios produce more heat compared to lean mixtures so higher peak temperatures are expected. When the effect of the intake temperature is considered, an increasing in the peak temperature is obtained as the intake temperature increases. In fact, higher intake temperatures expedite the species reaction rate by accelerating the destruction process of the fuel molecules. Therefore, the bonds making and breaking between the participating molecules will be enhanced dramatically. This observation is predicted in lean, rich and stoichiometric mixtures combustion with relatively the same effecting rate. Such conclusions are demonstrated clearly in Figure 72, which show the peak temperature as function of both of the equivalence ratio and the intake temperature. 


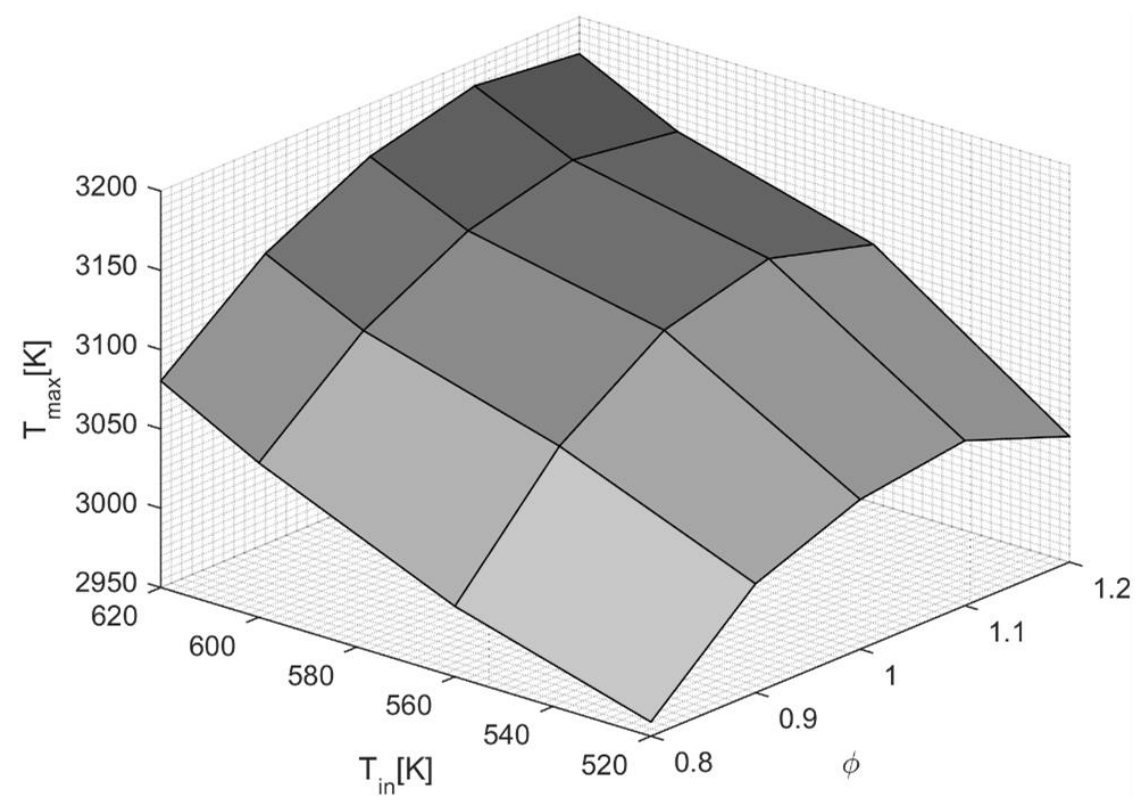

Figure 72 In-cylinder peak temperature at different intake temperature.

From emissions perception, the $\mathrm{NO}_{x}$ emissions level is not affected mostly by the intake temperature change as Figure 73 and Figure 74 indicate. It was shown that, those emissions are more in the case of the lean mixtures due to the plenty of $\mathrm{O}_{2}$ concentrations. Therefore, the availability of the $\mathrm{O}_{2}$ molecules is the dominant even if higher intake temperatures are applied. In fact, a rise in the intake temperature will speed up the $\mathrm{NO}_{x}$ formation rate instantaneously but it will not increase the overall NOx quantity. This also can be concluded by tracking the change of the NOx emission with the peak temperature. The difference between the lean and the rich mixtures in the peak temperature has much less effect the mixture composition. Chemically, a significant change in the reaction temperature can lead to more molecules impact. Therefore, more branches and bonds will be broken; this will give more chances for a new molecules formation like $\mathrm{NO}_{x}$ to be produced.

In Figure 75, CO emissions is illustrated at different equivalence ratios and intake temperatures. The figure indicates no effect on the $\mathrm{CO}$ levels due to the change in the intake temperature, which follows the same trend in the case of $\mathrm{NO}_{\mathrm{x}}$ emissions. Based on that, no change in the UHC level is expected also as those emissions are associated with the $\mathrm{CO}$ outcomes. As a general conclusion, the intake temperature seems to have a negligible effect on the emissions formation within the free-piston engine, but it has a noticeable effect on the engine performance. 


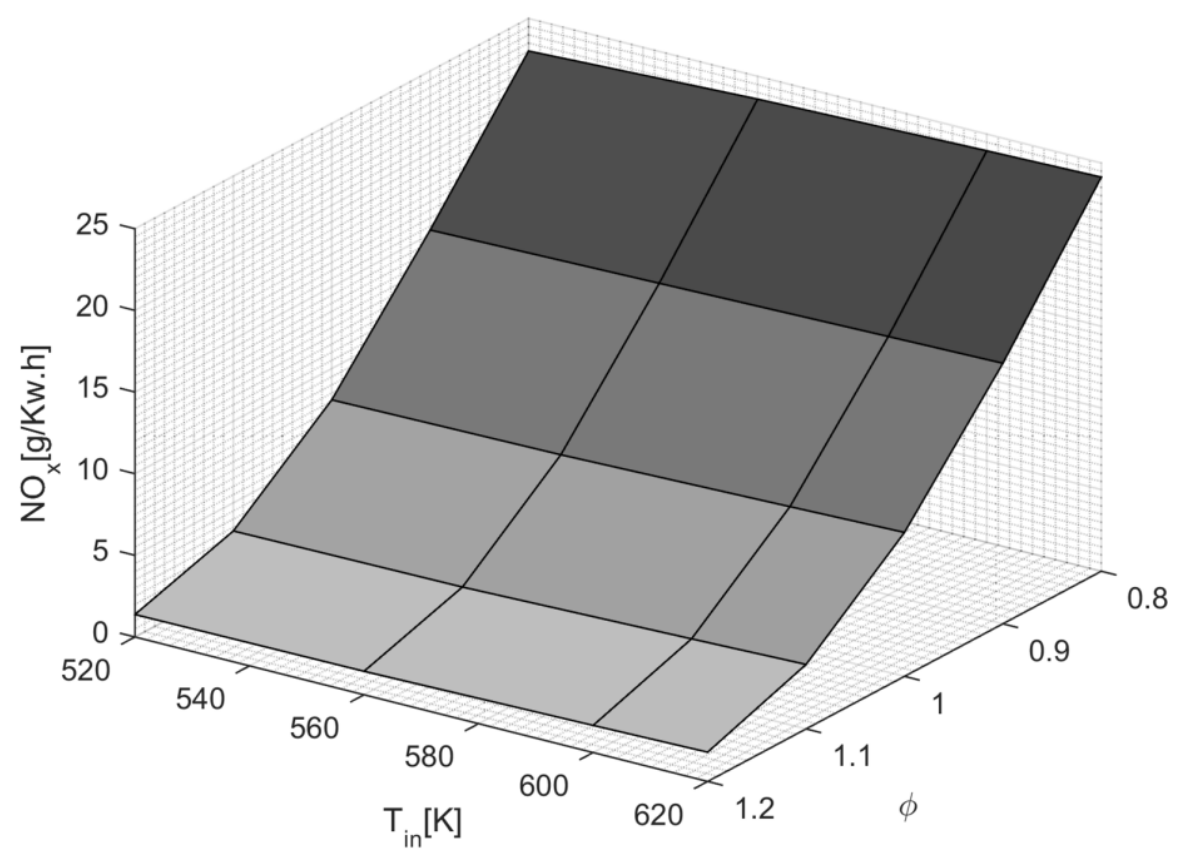

Figure $73 \mathrm{NO}_{\mathrm{x}}$ emissions at different intake temperatures.

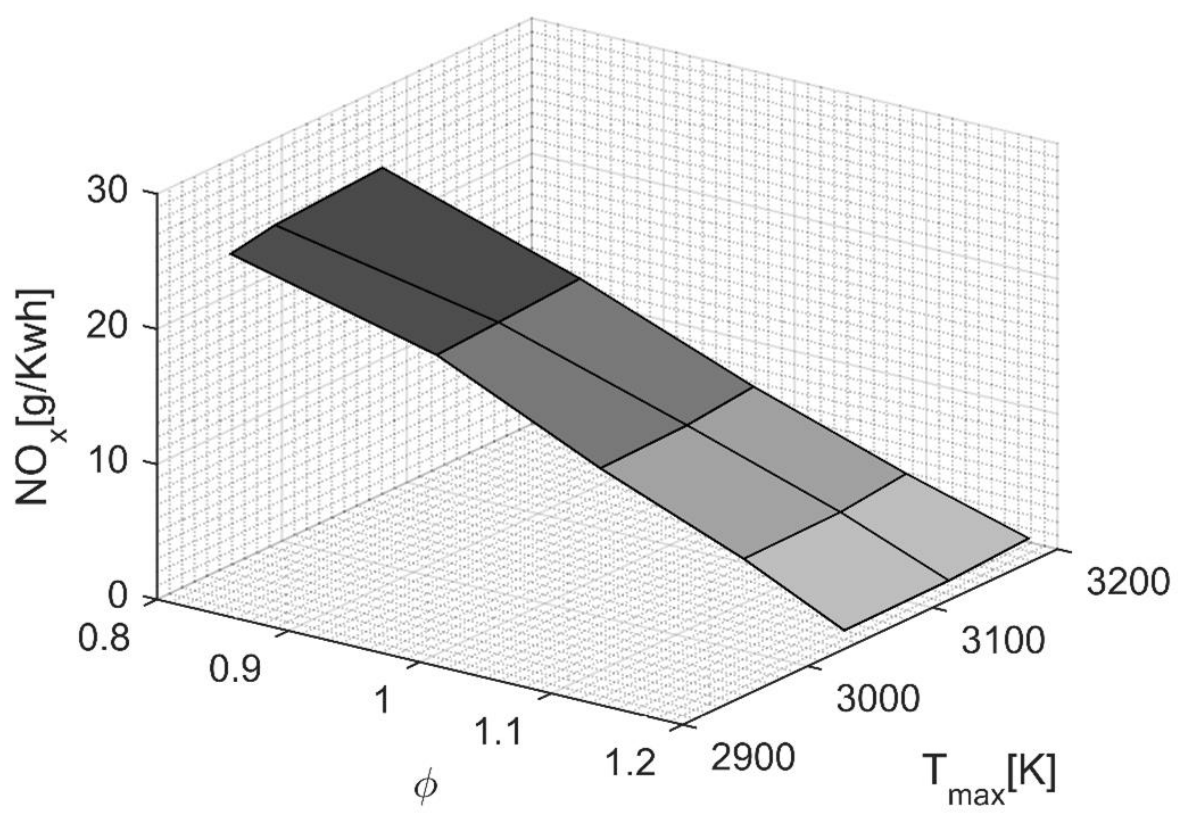

Figure 74 NOx variations with the maximum peak temperature. 


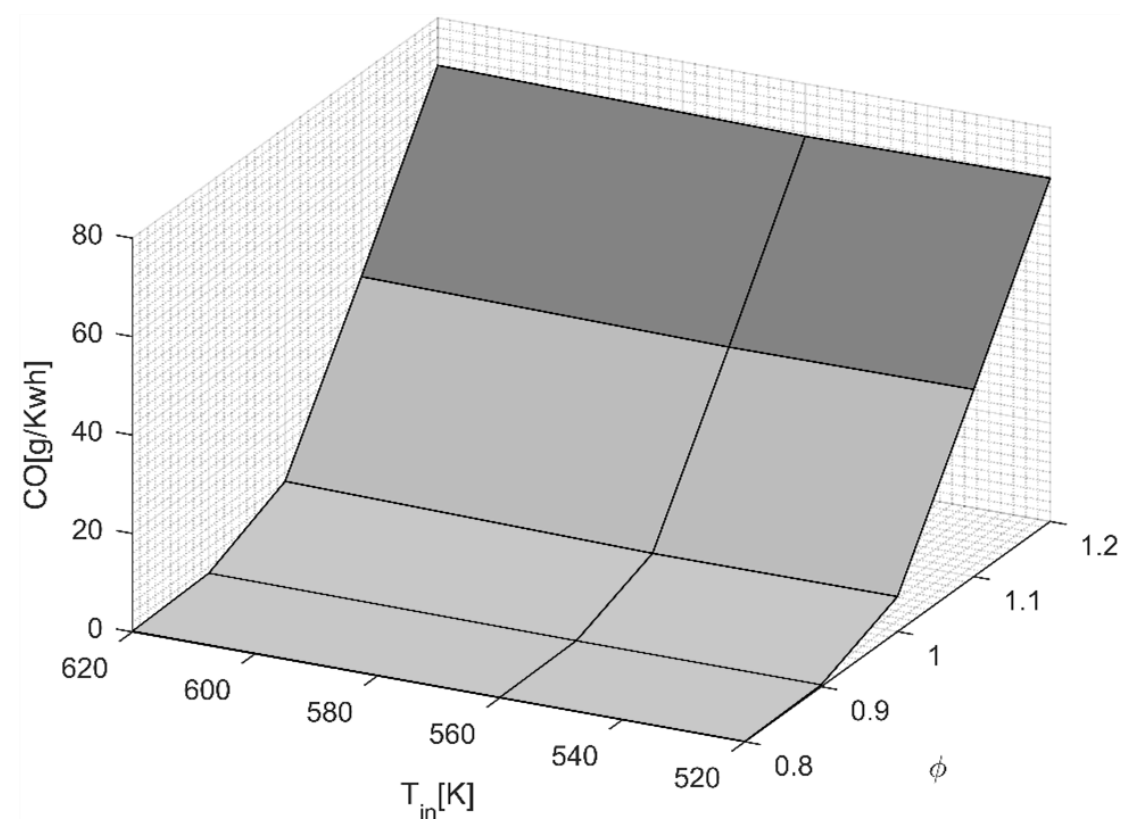

Figure $75 \mathrm{CO}$ emissions at different intake temperatures.

\subsection{Ultra-lean combustion:}

In the current research, a basic study in the effect of implementing ultra-lean mixtures $(\phi \leq 0.5)$ in the free-piston operation has been performed. Evaluating the effect of the ultra-lean combustion on the dynamics and the in-cylinder thermodynamic process is the main target of the study. All of the condition and parameters have been summarized in Table 6 but with different equivalence ratios.

Starting with the ignition delay and the HRR, Figure 76 represents the HRR produced by the freepiston engine at different low equivalence ratios. Within the ultra-lean range $(\phi \leq 0.5)$, the ignition delay is decreasing as the equivalence ratio decreases which is expected for lean combustion. The difference in the case of the ultra-lean combustion is the burning duration. This duration is increasing noticeably as lower equivalence ratios are implemented. Typically, the slower oxidation rate of the fuel molecules by the intake air is the main reason of this increase in the burning duration. In fact, the excessive air quantities in the ultra-lean charges act as diluters for the whole fuel-air charge, this dilution effect will decrease the reactions speed dramatically. In advanced cases, a full suppression of those reactions can happen leading to misfire in the combustion chamber. Finally, Figure 76 indicates very low HRR values when extra low equivalence ratios are applied. The limited fuel molecules compared to the air molecules is the reason for such low HRR values. 


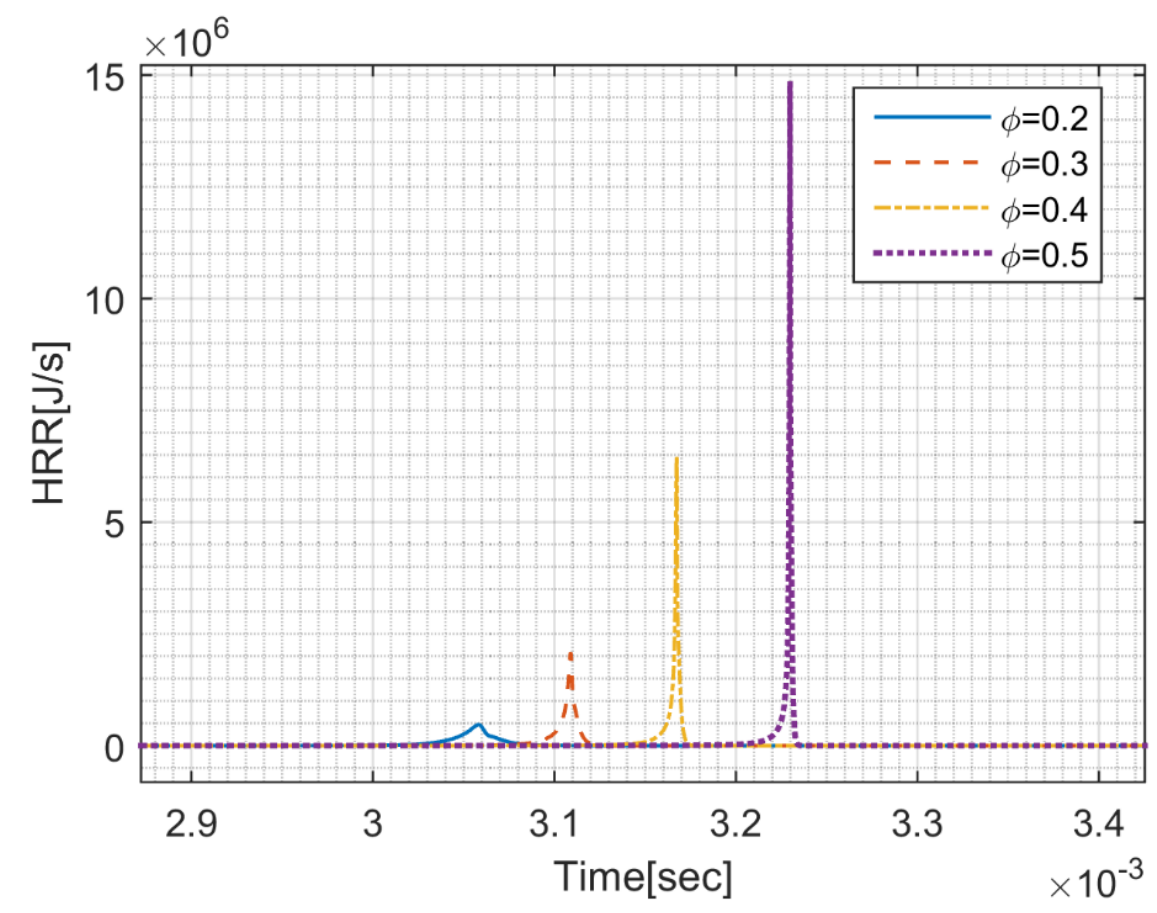

Figure $76 \mathrm{HRR}$ at different equivalence ratios (ultra-lean).

The in-cylinder pressure of the free-piston engine is presented in Figure 77 for different ultra-lean mixtures. It is noticed that, a slight decrease in the peak pressure is obtained as very low equivalence ratios are used. The ignition delay and the combustion duration are also identified for each equivalence ratio. The combustion event appears in the case of the extra low equivalence ratio as a gradual increasing in the pressure; in the other side, a sharp and sudden event is observed at higher equivalence ratios. The reason of that is the variations in the reactions rate between the fuel and air molecules due to the dilution effects. Theoretically, the small difference in the peak pressure will enhance the thermal efficiency of the engine by keeping the same combustion pressure but with less energy consumption. 


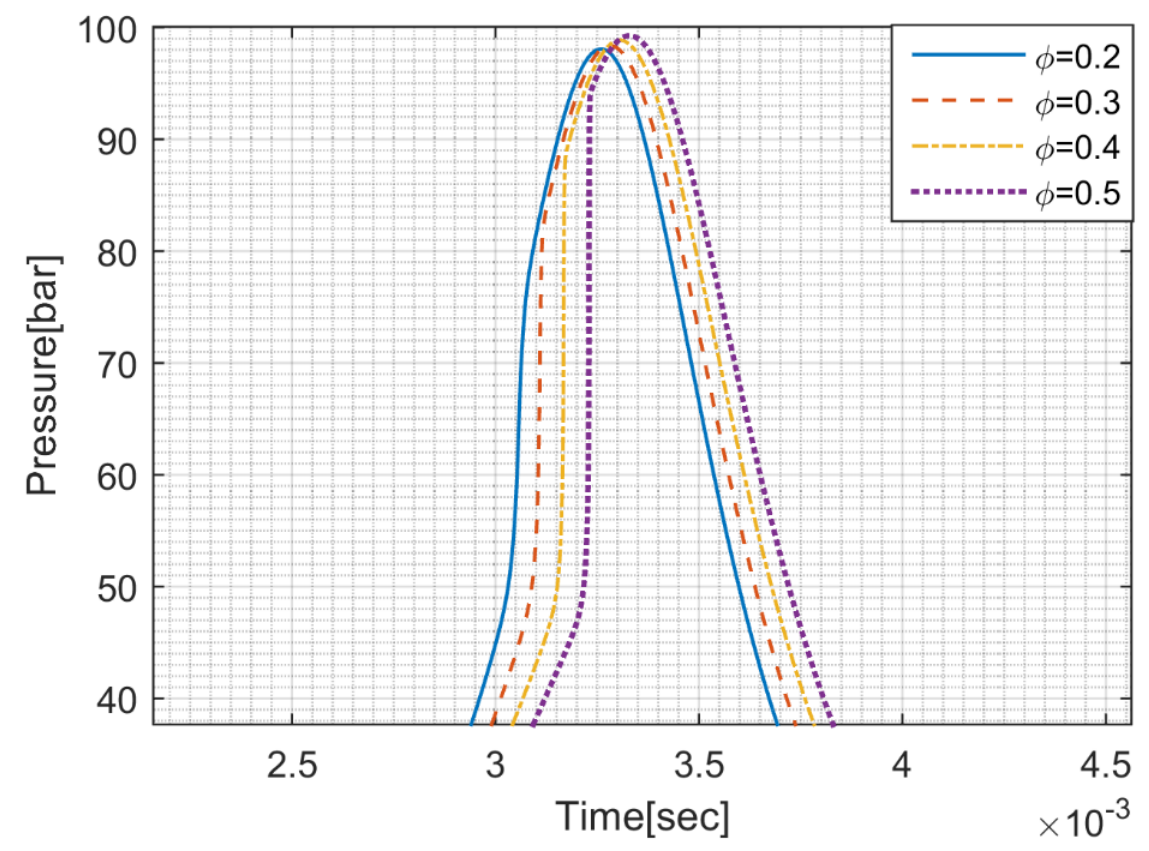

Figure 77 In-cylinder pressure at different equivalence ratios (ultra-lean).

Extra details can be obtained from Figure 78 where the in-cylinder pressure is presented as a function of the of the piston position. The difference in the burning duration is shown clearly in the figure. In the case of the extra low equivalence ratios, the ignition event is slower and it occurs smoothly at the end of the compression stroke. In the other side, a sharp and sudden pressure rise coupled with a short burning time are observed at higher equivalence ratios. Figure 78 demonstrates an important observation as well by showing the significant variations in the adverse work area. The adverse work becomes greater as the fuel-air mixture becomes leaner; this indicates that the piston has higher kinetics energy compared to the energy that is delivered to the alternator. At the same time, the springs store the same energy regardless the mixture case since they have constant stiffness values. Such increasing in the kinetics energy refers to the disability of the engine to absorb the piston energy completely during the expansion stroke. The early ignition events and the reduction in the applied load explain the changes in the adverse work values. In fact, the operation stability requires the system to satisfy the initial conditions to end of the cycle. The controller changes the load based on the available piston energy; but at the same time, it works on achieving the cycle stability by seeking the same initial conditions at the end of cycle. The variations in the adverse work require extra control; this makes the use of very ultra-lean mixtures impractical in many cases due to these difficulties.

In Figure 79, the compression ratio for different ultra-lean mixtures is presented, the compression ratio is decreasing dramatically as the mixture becomes far from the ultra-lean region. This reduction in the compression ratio shows the ability of the ultra-lean mixtures in extending the compression ratio range within the $\mathrm{HCCl}$ operation. The chemical composition of the ultra-lean mixtures will assist the rise in the compression ratio by the producing a diluted mixture using the excess air content. The resulted charges will show a high reduction in the reactions rate. As a result, the mixture will require higher 
compression to reach the ignition point. The self-ignition temperature will also be higher due to this high compression ratio.

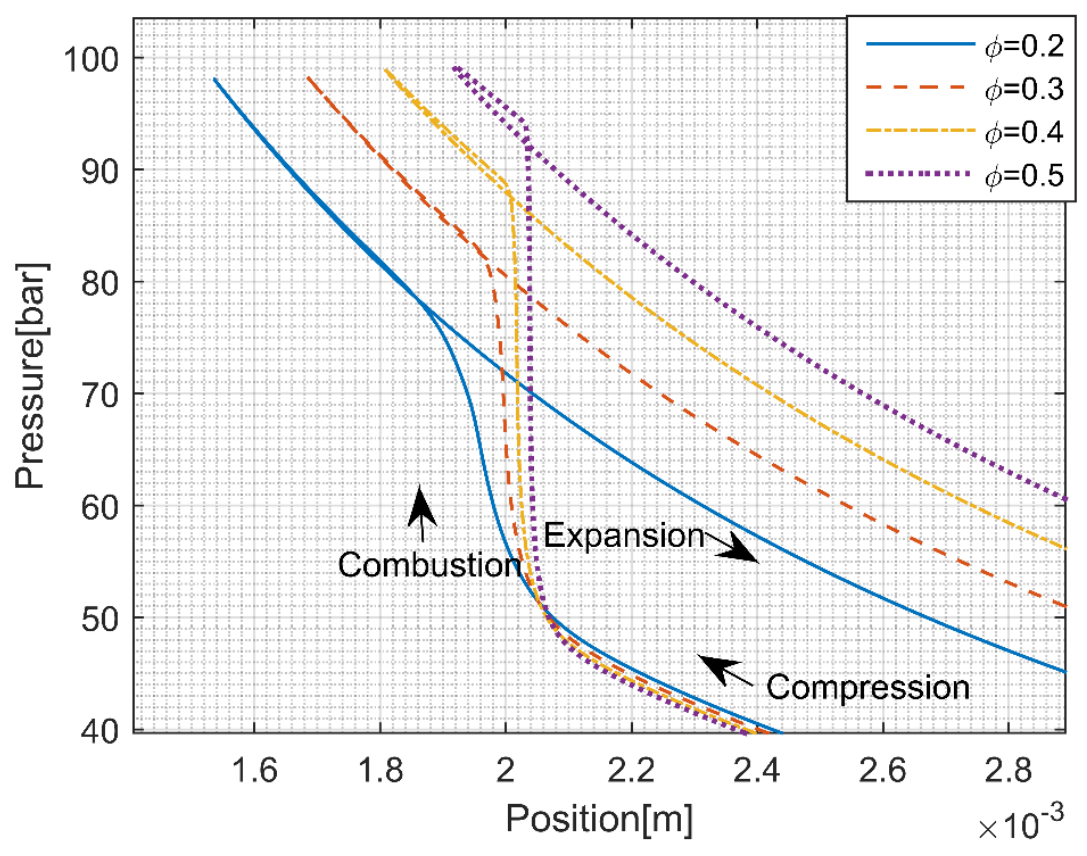

Figure 78 Pressure-position diagram at different equivalence ratios (ultra-lean).

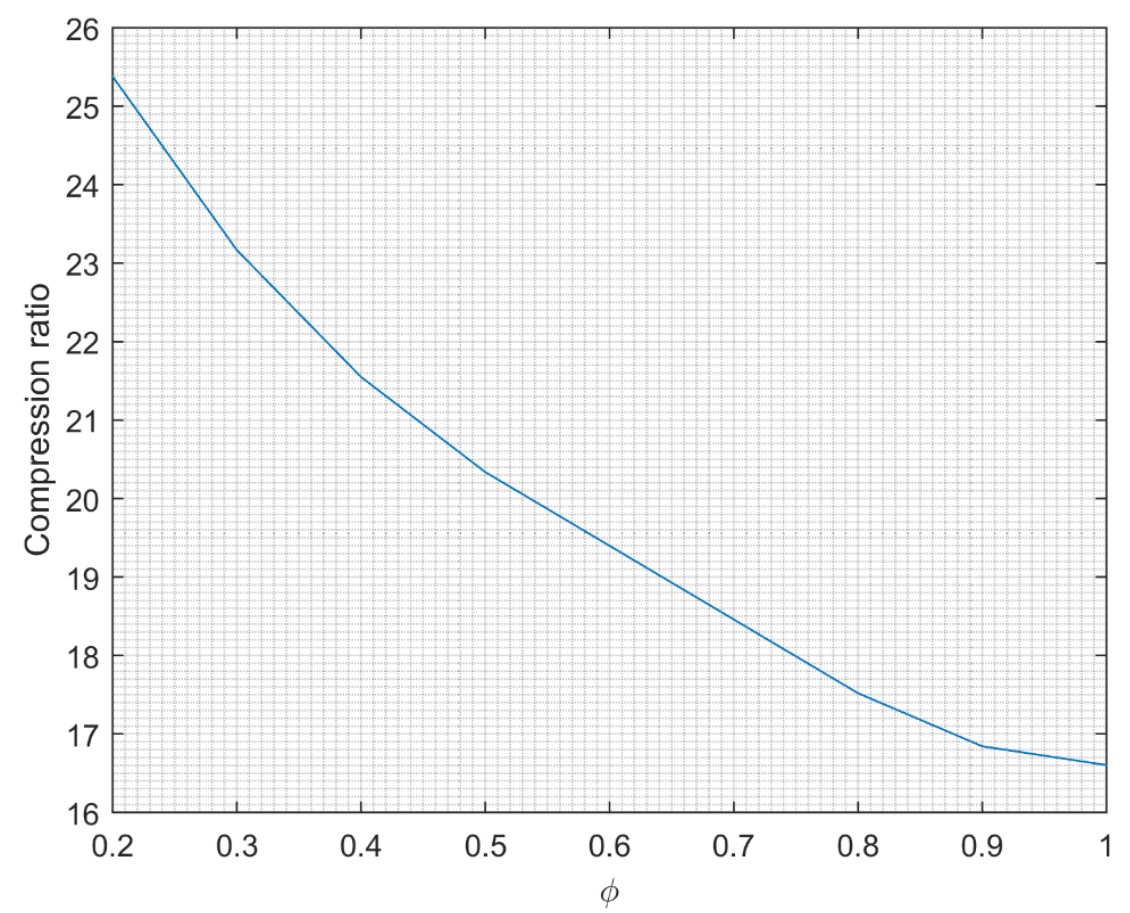

Figure 79 Compression ratio at different equivalence ratios (ultra-lean). 
The in-cylinder pressure variations are always associated with the variations in the work output ratio. Figure 80 describes the alternator work output of the free-piston engine as a function of the equivalence ratio. The figure indicates that the alternator is more efficient when ultra-lean mixtures are implemented. However, low equivalence ratios $(\phi<0.3)$ will show a reduction in the work output ratio. Moderate ultralean mixtures $(0.3<\phi<0.5)$ have the optimal work percentages while mixtures that exceeded that limit show lower work outputs. Although the ultra-lean charges appear more efficient, but still their load range is limited within the light range. Technically, the alternator determines the transferring of the combustion energy to a useful work. Therefore, since ultra-lean mixtures have great adverse work, this will limit their operation to be only within the light load applications.

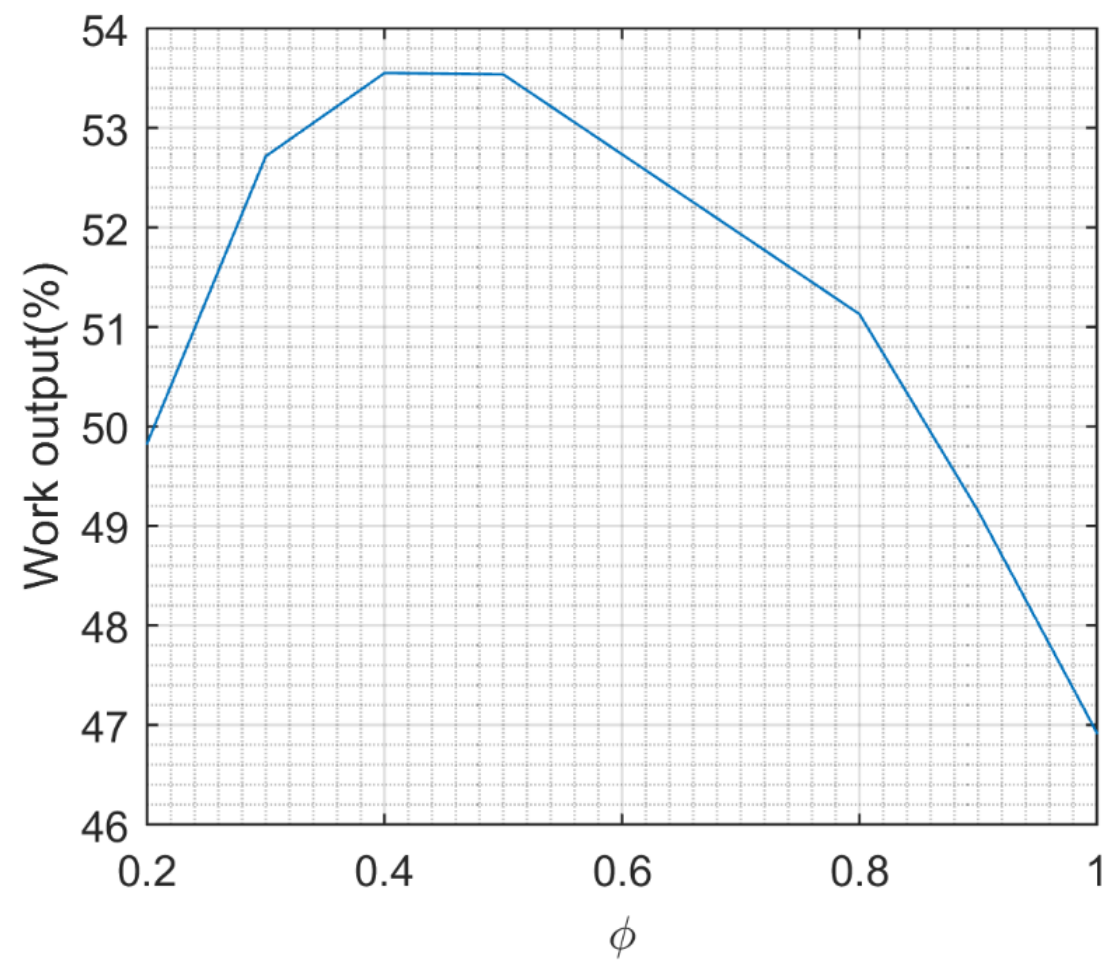

Figure 80 Work output ratio vs. equivalence ratio (ultra-lean).

By considering the effect of the ultra-lean implementation on the combustion temperature, it was found that the peak temperature is decreasing significantly, as the mixture becomes leaner. These results are illustrated in Figure 81 clearly. The obtained reduction in the HRR, which is observed at low equivalence ratios, explains the low combustion peak temperatures. In addition, the excess air within the intake charge is acting like a coolant during the fuel-air burning. The overall effect will reduce the species reactions speed and their burning time. Usually, at similar conditions high heat losing rates are observed due to the plenty of time that is available for the gradual heat transfer process. Emissions like $\mathrm{NO}_{x}$ will have some variations in their formation rate with the combustion temperatures. Low peak temperatures will indicate low $\mathrm{NO}_{x}$ emissions outcomes and high peak temperatures refer to high NOx formation. In general, the results show that a significant peak temperature reduction is necessary to decrease the $\mathrm{NO}_{x}$ formation rate in a practical manner. 


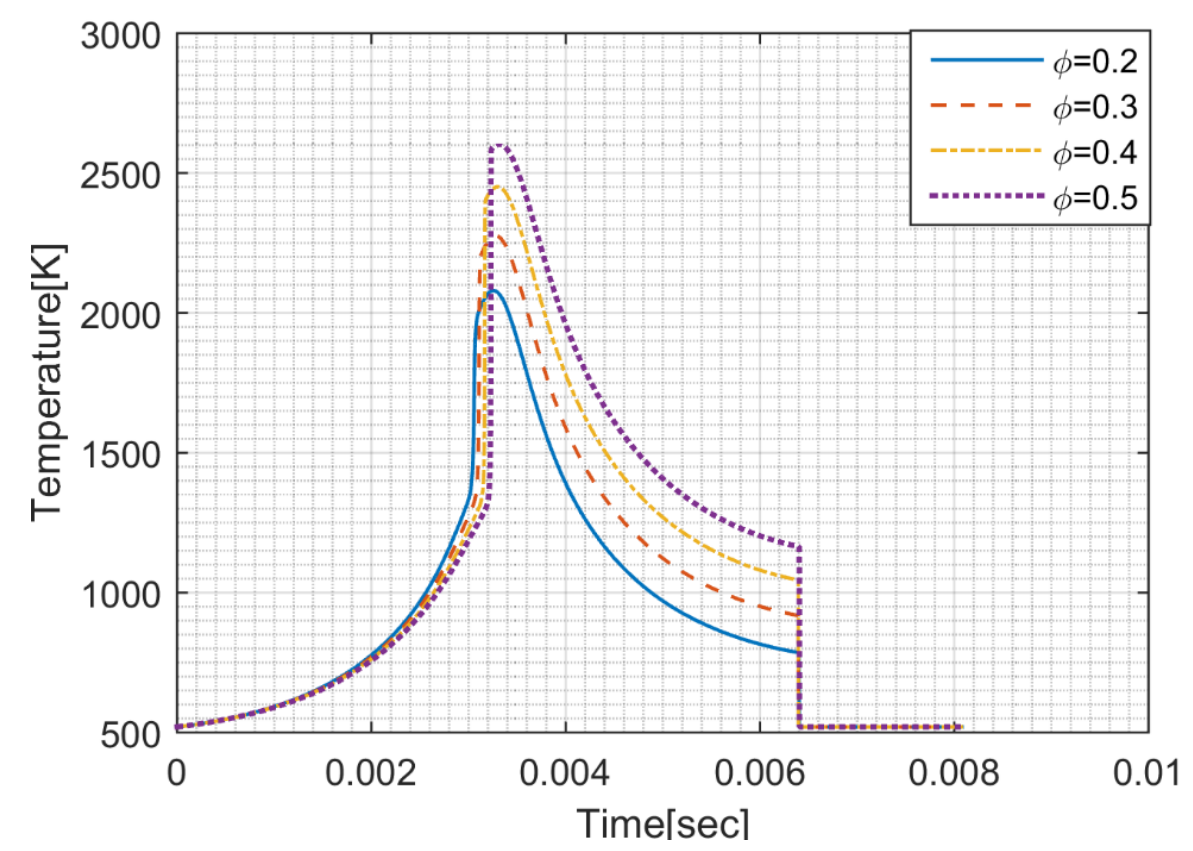

Figure 81 In-cylinder temperature at different equivalence ratios (ultra-lean).

Lean mixtures that are close to the stoichiometric conditions have high $\mathrm{NO}_{x}$ emissions. The complete burning of the intake charges provides the heat necessary to form the $\mathrm{NO}_{\mathrm{x}}$ molecules; in addition, the excess $\mathrm{O}_{2}$ content at that level will also assist the oxidation of any $\mathrm{N}$ atoms later on. In the case of the ultra-lean mixtures, a very sharp reduction in the $\mathrm{NO}_{x}$ formation is achieved by reducing the combustion peak temperature dramatically. $\mathrm{N}_{2}$ and $\mathrm{O}_{2}$ molecules reactions will be limited when relatively low temperatures are presented. Figure 82 demonstrates this fact clearly, where the $\mathrm{NO}_{\mathrm{x}}$ is presented at different low equivalence ratios. Figure 82 includes the lowest $\mathrm{NO}_{x}$ level that is obtained at rich conditions for comparison purposes. A sharp drop in the $\mathrm{NO}_{x}$ formation rate is observed as extra low equivalence ratios are applied, low values like $(\phi=0.3)$ results with similar formation rate to this found in the case of the rich mixture ( $\phi=1.2)$. Furthermore, less equivalence ratios will show near zero $\mathrm{NO}_{x}$ emission levels. Such results reflect the effectiveness of using ultra-lean combustion in $\mathrm{NO}_{x}$ emissions reduction. However, the mentioned difficulties in controlling the system performance will limit the use of the ultra-lean combustion.

$\mathrm{CO}$ emissions under the effect of the ultra-lean combustion have been also investigated. Figure 83 presents the $\mathrm{CO}$ emissions formation rate at different ultra-lean values. As indicated in the figure, the $\mathrm{CO}$ formation rate is almost zero during the cycle except at ignition event. The absence of the adequate fuel molecules and the availability of excess air will restrict any incomplete burning process; therefore, no chances to produce CO molecules are presented. In similar way, the UHC emissions will be in the minimum levels since low CO levels indicate low UHC usually as the last are strongly defined by the CO formation factors. 


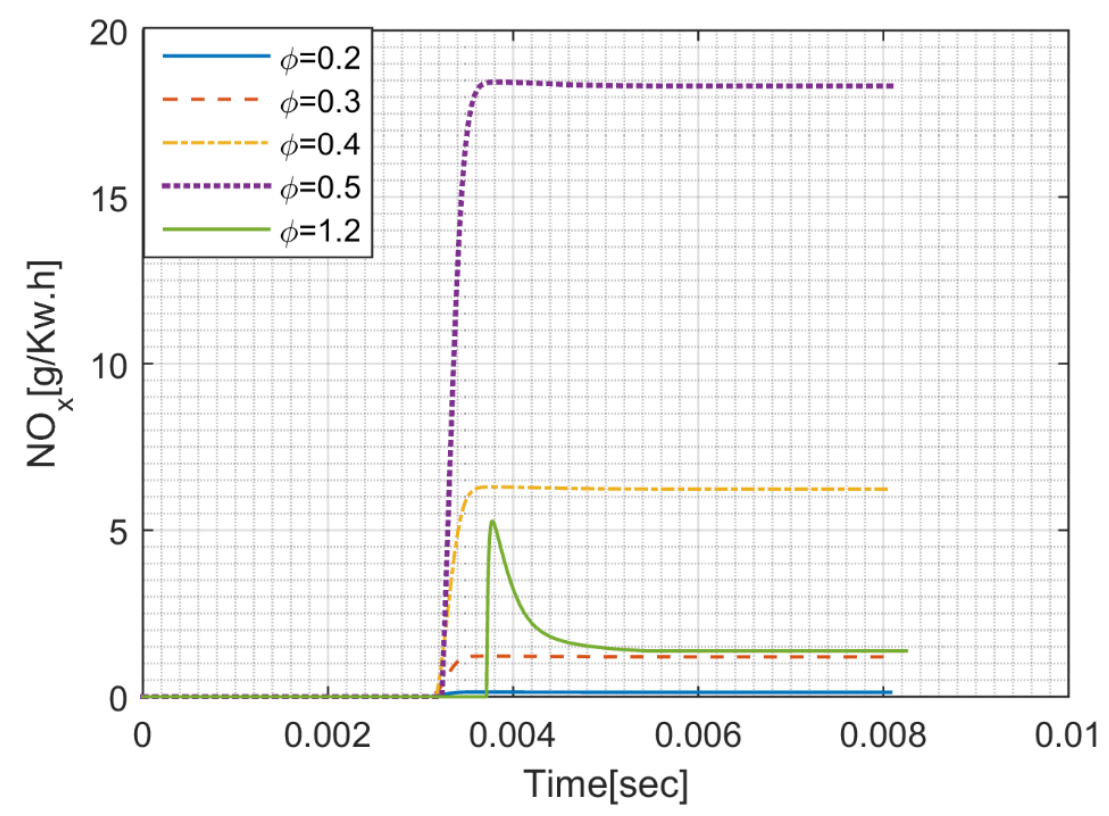

Figure $82 \mathrm{NO}_{\mathrm{x}}$ emissions at different equivalence ratios (ultra-lean).

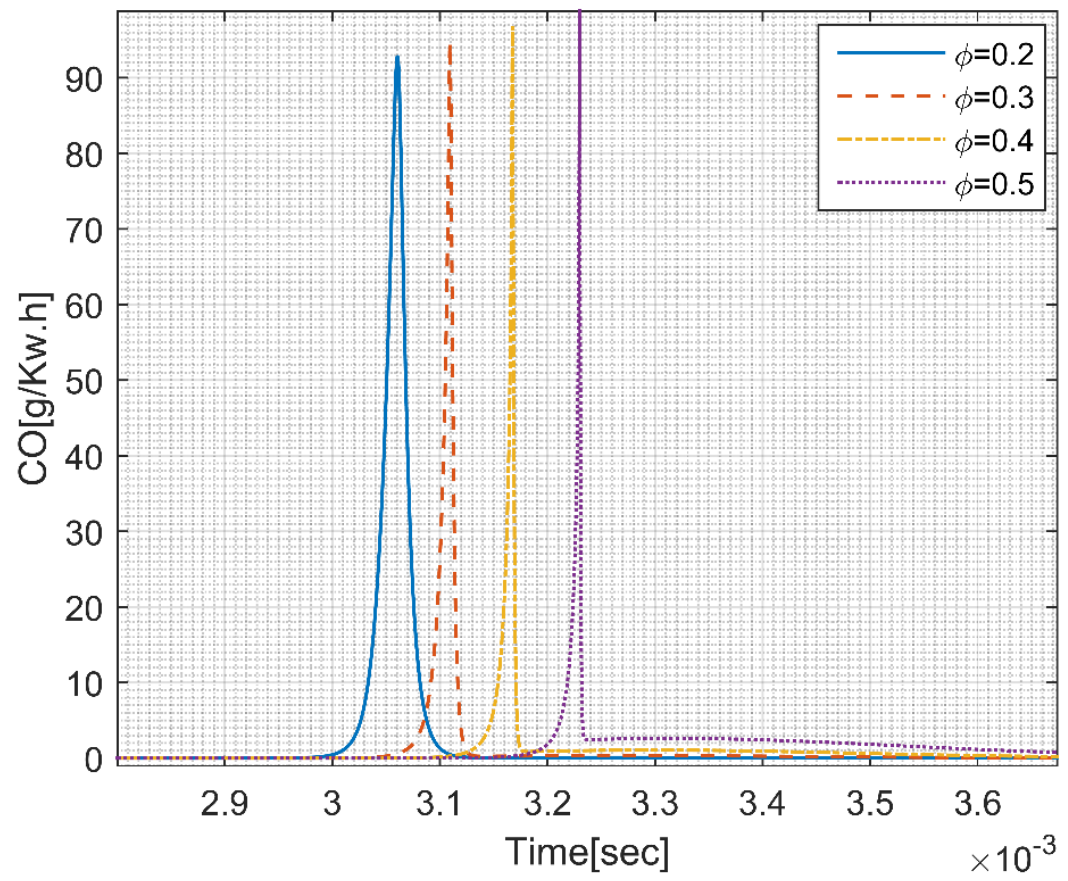

Figure $83 \mathrm{CO}$ emissions at different equivalence ratios (ultra-lean). 


\section{Chapter 7. Fuels and fuels mechanisms}

Piston dynamics and the in-cylinder thermodynamics are very sensitive to HRR where any changes within the free-piston engine cycle can affect the overall performance. This fact introduced the need of including the fuel type under the study scoop. In addition, evaluating the engine performance under the influence of different types of fuel will define the best suitable conditions for the engine. The importance of the fuel investigation is not limited by the heating value only, it includes the durability and the reliability of the engine performance as well [97].

\subsection{Fuel variations effect}

In order to investigate the performance of the free-piston engine model under the influence of different fuels, the fuels listed in Table 7 have been applied under the specified conditions where the fuels have been applied under the same masses and intake conditions.

Table 7 Fuel types and initial conditions.

\begin{tabular}{cccccc}
\hline Fuel & Air to fuel & Air-fuel mass $\mathbf{( g )}$ & $\boldsymbol{\phi}$ & $\mathbf{T}_{\mathbf{0}}(\mathrm{K})$ & $\mathbf{P}_{\mathbf{0}}(\mathrm{Kpa})$ \\
\hline Methane (CH4) & 17.25 & 0.0156 & 1 & 560 & 101 \\
\hline Ethanol (C2H6O) & 9.00 & 0.0174 & 1 & 560 & 101 \\
\hline Propane (C3H8) & 15.68 & 0.0174 & 1 & 560 & 101 \\
\hline DME (CH3OCH3) & 9.00 & 0.0174 & 1 & 560 & 101 \\
\hline n-heptane (C7H16) & 15.18 & 0.0180 & 1 & 560 & 101
\end{tabular}

Starting with the heat release rate, Figure 84 illustrates the HRR of Methane, Ethanol, Propane, DME and $\mathrm{n}$-Heptane combustion process. Those fuels have been discussed within the literature review chapter. Two main features can be concluded out of Figure 84, the ignition delay and the HRR value. In the case of the ignition delay, each fuel shows a unique ignition where some fuels as DME ignites very early while others as Methane has a late ignition event. Fuels like Propane and $\mathrm{n}$-heptane shows intermediate ignition delay. Obviously, the variations in the ignition delay refers to the unique structure of each fuel. In addition, the nature of the free-piston engine assists the uniqueness of the fuel ignition by considering the moving of the piston under the chemical reactions solely. The resistance of the fuel to knock was discussed before by introducing the Methane number. This number is an index for each fuel ignition resistance based on its content of Hydrogen and Carbon. For the mentioned fuels, DME has the lowest Methane number then Ethanol and of course, Methane will have the highest value. The second feature is the HRR value; in this case, $n$-heptane has the highest HRR while Ethanol has the lowest value. The nature of the exothermic and endothermic reactions and the difference in the enthalpy of formation in those reactions are the main reasons for such variations in HRR. 


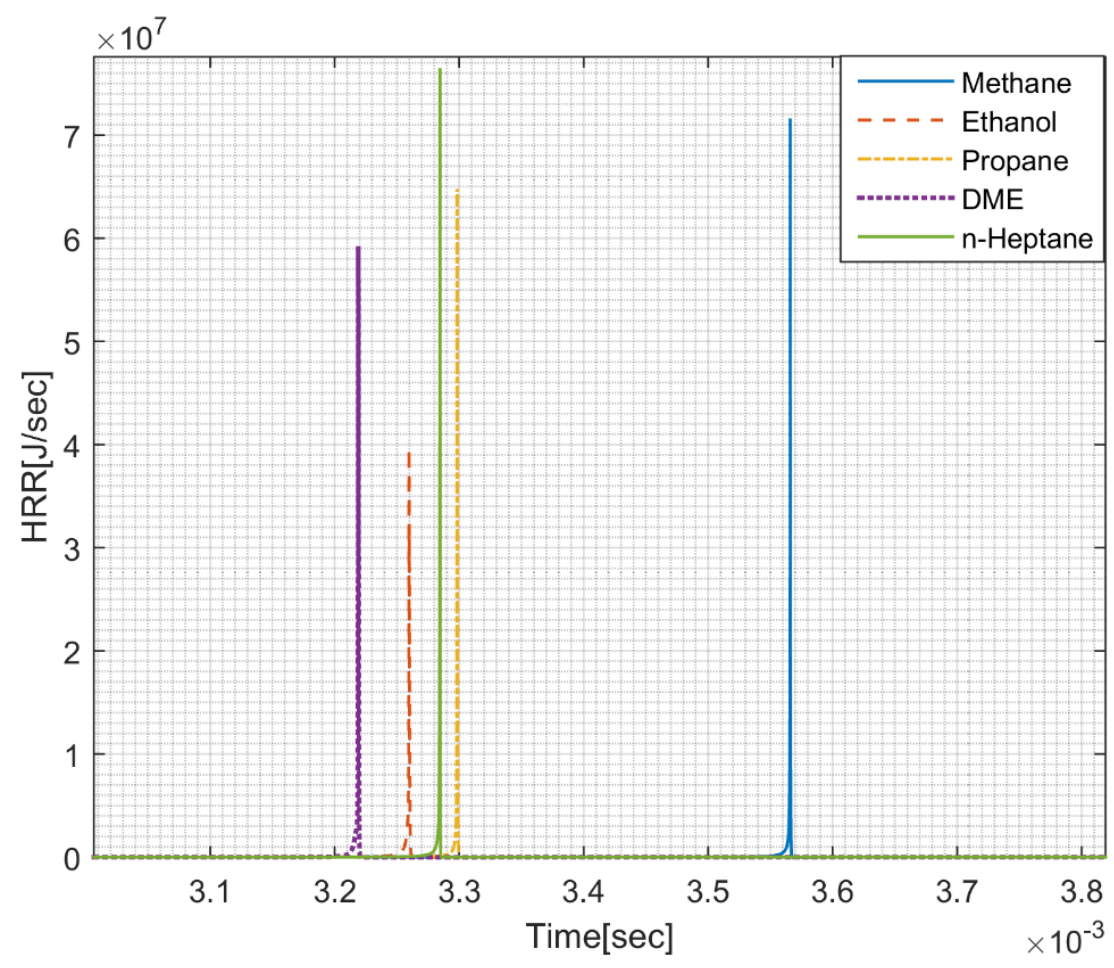

Figure 84 HRR for different fuel types.

The in-cylinder pressure for each fuel is demonstrated in Figure 85. The figure presents the ignition event as a sharp and sudden rise in the pressure at the end of the compression stroke. Fuels which have low ignition delay like DME ignites first; however, fuels like Methane will stay longer until ignition process is happen. The peak pressure of combustion varies from fuel to fuel as well. n-heptane shows the highest peak pressure value while all of the remaining fuels have very close values. It is known that the pressure diagram will give an estimation about the system efficiency. The relation between the pressure and the piston dynamics as it is shown in Figure 86 describes the in-cylinder pressure as a function of the piston position. Figure 86 was zoomed near the TDC region for the importance of combustion process. Low knock resistance fuel will have shorter compression stroke in general. However, fuels like $n$-heptane show longer strokes; this reflects the independency between the ignition delay and the ignition location. All of the mentioned fuel except Methane ignited at the TDC position. Methane has the ignition after the TDC position because of its high knock resistance. The fuels that have TDC ignition events show smooth transfer from the compression stage to the combustion process. The combustion process cannot be defined specifically for each fuel. The difference in the TDC location leads to the conclusion that the ignition in the free-piston engine is unique for each fuel. This fact differentiates the free-piston engine from the conventional one where the ignition can be controlled to a specific time. The variations in the combustion process are reflected also in the adverse work area for each fuel cycle. Fuels like Ethanol show large adverse work areas while Methane for example does not show completely. It was found that the system control requires the treatment of the adverse work area in order to achieve the stability. Less intake temperatures and more near stoichiometric burning conditions were among the techniques to reduce this work area. 


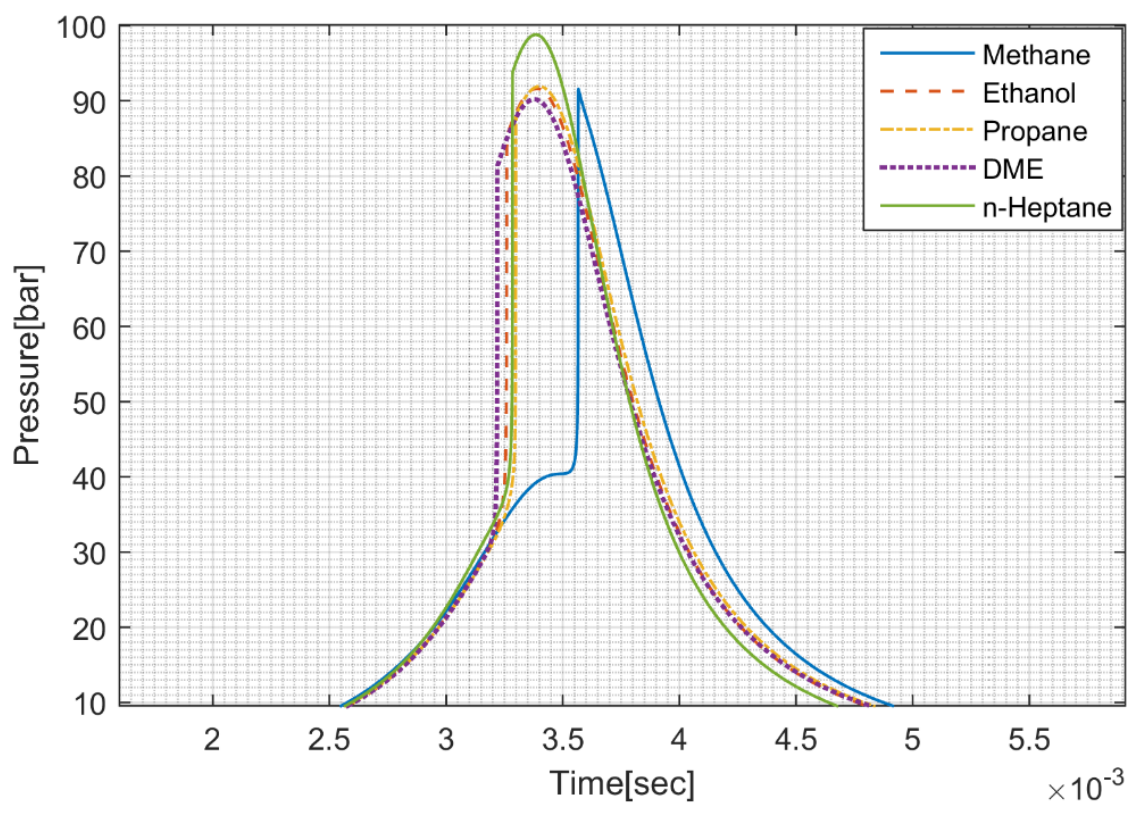

Figure 85 In-cylinder pressure for different fuels.

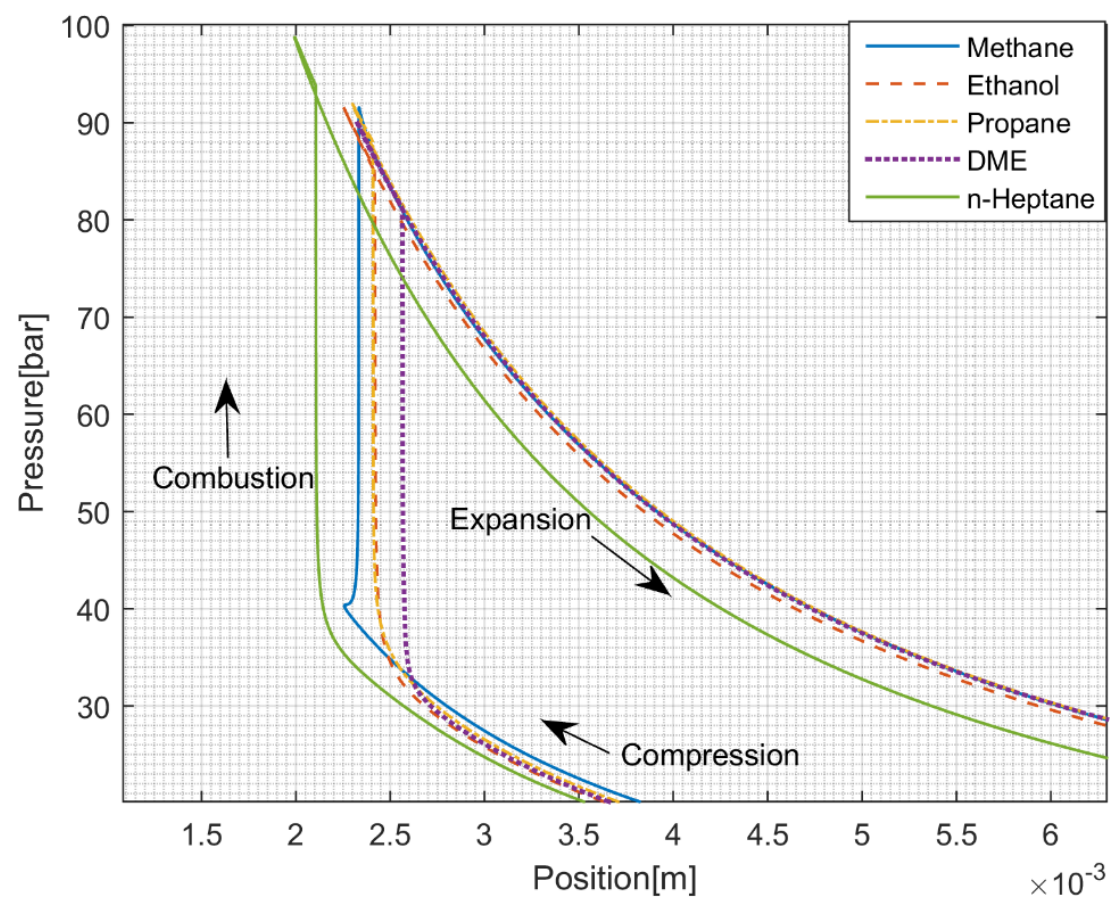

Figure 86 Pressure-Position diagram for different fuel types.

Changing the fuel can affect the engine compression ratio as shown in Figure 87. The compression ratio is very close for all of the fuels except for $n$-heptane where it is greater. The trend shown in the figurer seems to be similar to the peak pressure trend. In many other cases like the EGR and equivalence ratio, no similarities in the behavior were found. 


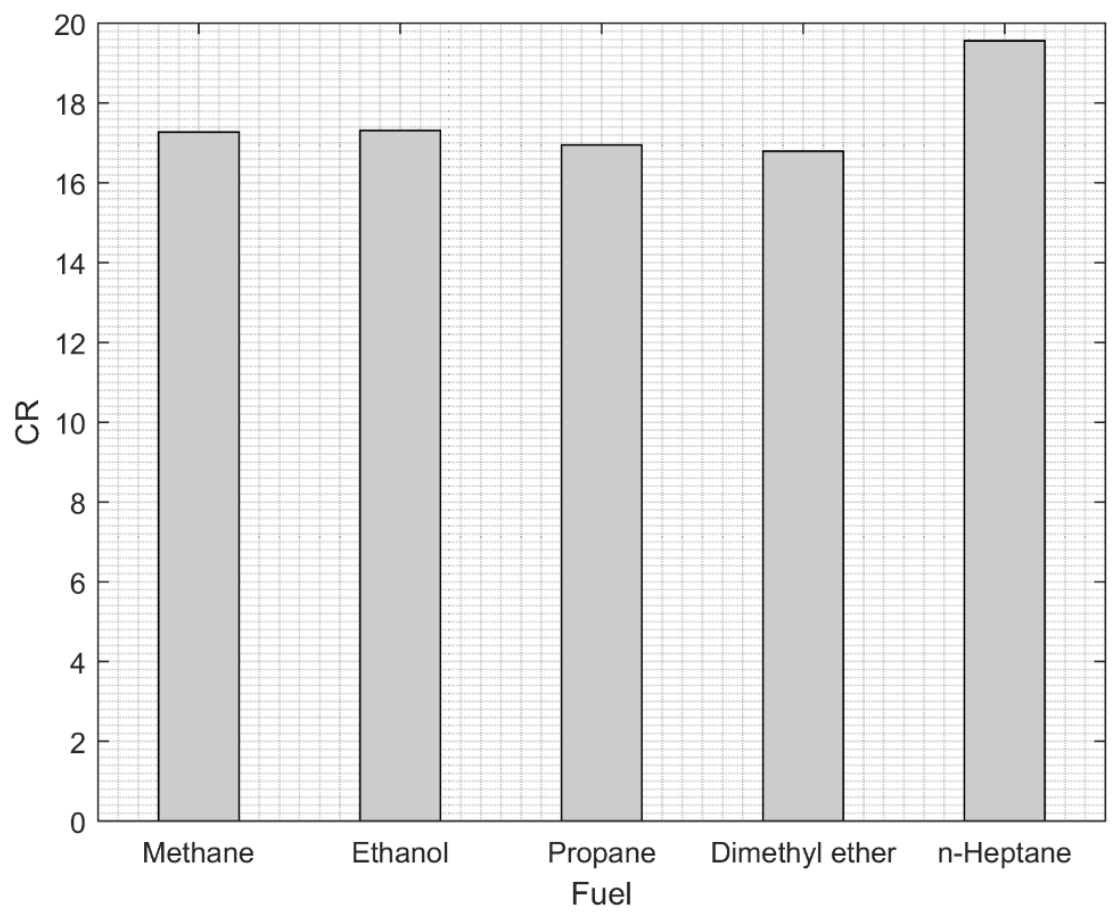

Figure 87 Compression ratio for different fuel types

Figure 88 represents the in-cylinder temperature of the free-piston engine under the influence of different fuel types. Starting with the self-ignition temperature of each fuel, this temperature is the highest for Methane while all the other fuels have less self-ignition temperature. In the case of the peak temperature, $\mathrm{n}$-heptane shows the lowest peak temperature among all the presented fuels, which share a close peak temperature values. Typically, the difference in the peak temperature refers to many reasons. The self-ignition temperature and the burning duration are the most effective factors. Fuels like $n$-heptane for example show low ignition temperature and short burning time compared to fuels like Methane.

Many of the mentioned parameters showed a sensitivity to applied fuel. As a result, the free-piston engine work output will also have some variations. In Figure 89, the work output ratio of the free-piston engine is presented. There is a slight difference in the work output ratio as different fuel is applied. The results that indicate that Methane is the most efficient fuel show also close work output ratio for the other fuels. Similar work outputs support that ability of the engine model to work under the influence of different fuels. In such cases, the converting from using conventional fuels to more environmental ones will be an efficient way to reduce the emissions as well. 


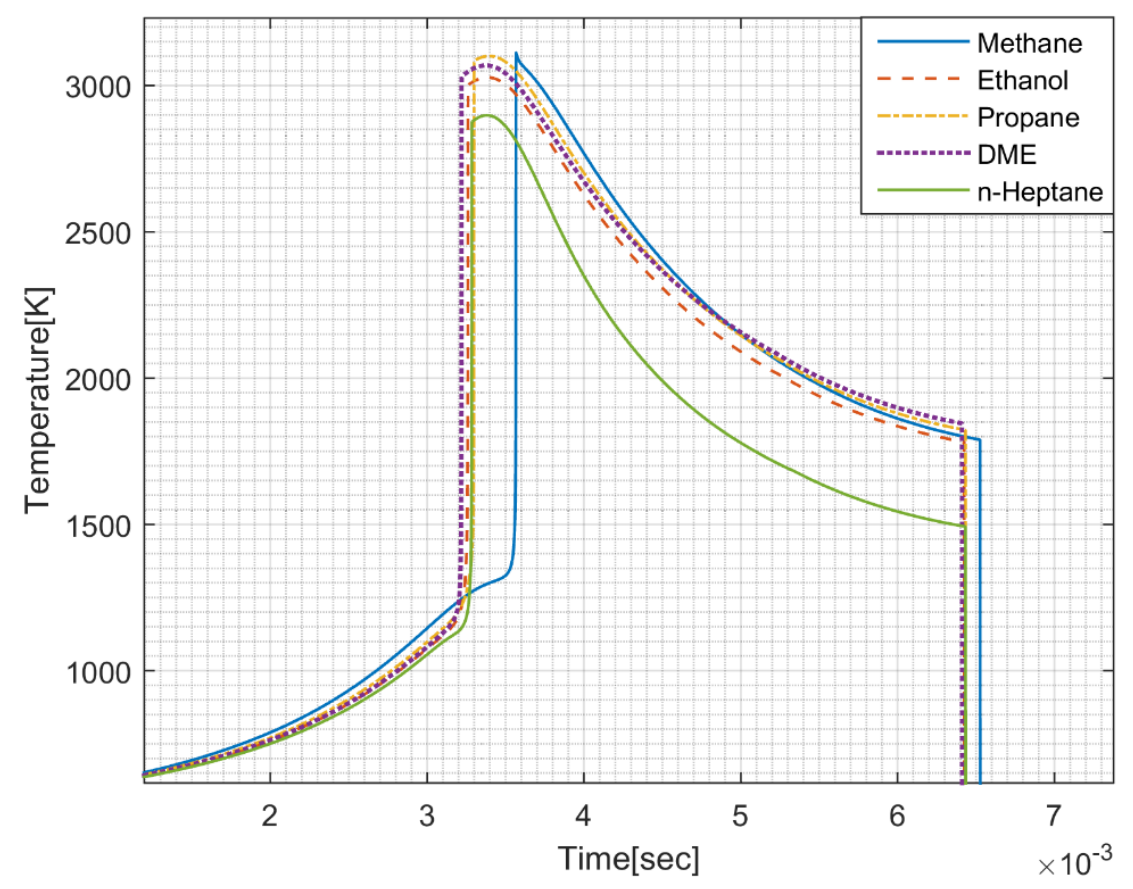

Figure 88 In-cylinder temperature for different fuel types.

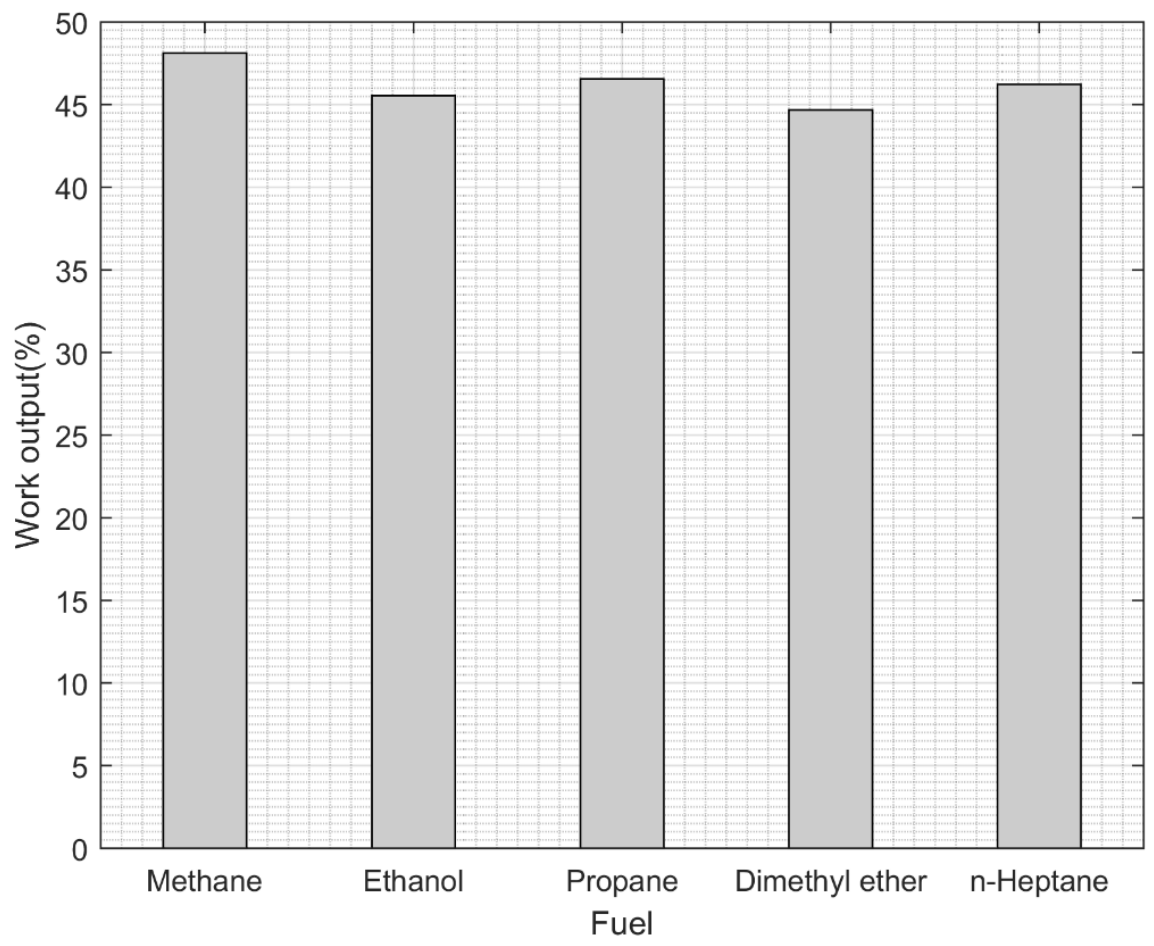

Figure 89 Work output ratio for different fuel types. 


\subsection{Fuel mechanism}

In the case of the free-piston engine where the thermodynamics and the dynamics are coupled tightly, it is expected to have performance sensitivity toward the fuel mechanism. The unconstrained nature of the free-piston engine in addition to the $\mathrm{HCCl}$ nature will be the main factors that should be considered if a reduced mechanism will be implemented. In this study, a quick evaluating of the free-piston model has been performed under the effect of different detailed and reduced mechanisms. Three main fuels have been implemented in both aspects (detailed and reduced). Methane is presented by two mechanisms, GRI3.0 [113](53 species and 325 reactions) as the full detailed mechanism and a reduced mechanism by Lu and Law [137] (36 species and 222 reactions). Ethanol is used as well, a mechanism by Marinov [138] (61 species and 396 reactions) represents the detailed mechanism while the reduced one is by Zhang et al [139] ( 40 species and 241 reactions). Finally, $n$-heptane is introduced by a full mechanism (556 species and 2540 reactions) done by Curran et al [140]. In the other side a reduced mechanism is implemented as well, the mechanism (159 species and 770 reactions) was developed by Seiser et al [141]. Based on their developers, the reduced mechanisms had been approved for $\mathrm{HCCl}$ combustion process where the ignition delay and combustion phase are the main concerns of those mechanisms. In the other side, the reduced mechanism does not include emissions calculations, so no emissions comparison is considered in this section. The selected fuels mechanisms are listed in Table 8 where each mechanism species and reactions numbers are listed by including the detailed and the reduced numbers.

The mechanisms have been applied to the free-piston engine model of this research and their results are shown in the figures below. Starting with the ignition delay, the HRR is illustrated Figure 90; as the figure shows, there is a slight difference between the ignition delay in the case of the reduced mechanisms. It is seen that the fuel-air mixture is taking more time to ignite in the case of the reduced mechanism for all of the presented fuels. The difference in the ignition delay can be explained by the reactions, which were reduced in the reduction process of the mechanism. The nature of the exothermic reactions plays a significant role in detraining the ignition temperature, in the case of the reduced mechanisms, many reactions from the detailed mechanism were combined and replaced with less accurate ones. Depending on the nature of those reactions and the number of the reduction steps, the change in the ignition delay will vary.

In this section, different fuel mechanisms have been applied to the engine model in order to predict the impact of the species and reactions reduction on the overall performance. The fuel mechanisms illustrated in Table 8 have been used under the listed conditions.

Table 8 Detailed and reduced mechanisms of some famous fuels.

\begin{tabular}{|c|c|c|c|c|c|c|c|c|c|}
\hline \multirow[b]{2}{*}{ Fuel } & \multicolumn{2}{|c|}{ Detailed Mechanism } & \multicolumn{2}{|c|}{ Reduced Mechanism } & \multirow[b]{2}{*}{ Air to fuel } & \multirow[b]{2}{*}{$\begin{array}{l}\text { Air-fuel } \\
\text { mass (g) }\end{array}$} & \multirow[b]{2}{*}{$\phi$} & \multirow[b]{2}{*}{$\mathrm{T}_{0}(\mathrm{~K})$} & \multirow[b]{2}{*}{$\mathrm{P}_{0}(\mathrm{Kpa})$} \\
\hline & species & reactions & Species & reactions & & & & & \\
\hline Methane & 53 & 325 & 36 & 222 & 17.25 & 0.0156 & 1 & 560 & 101 \\
\hline Ethanol & 61 & 396 & 40 & 241 & 9.00 & 0.0172 & 1 & 560 & 101 \\
\hline n-heptane & 556 & 2540 & 159 & 770 & 15.18 & 0.0180 & 1 & 560 & 101 \\
\hline
\end{tabular}




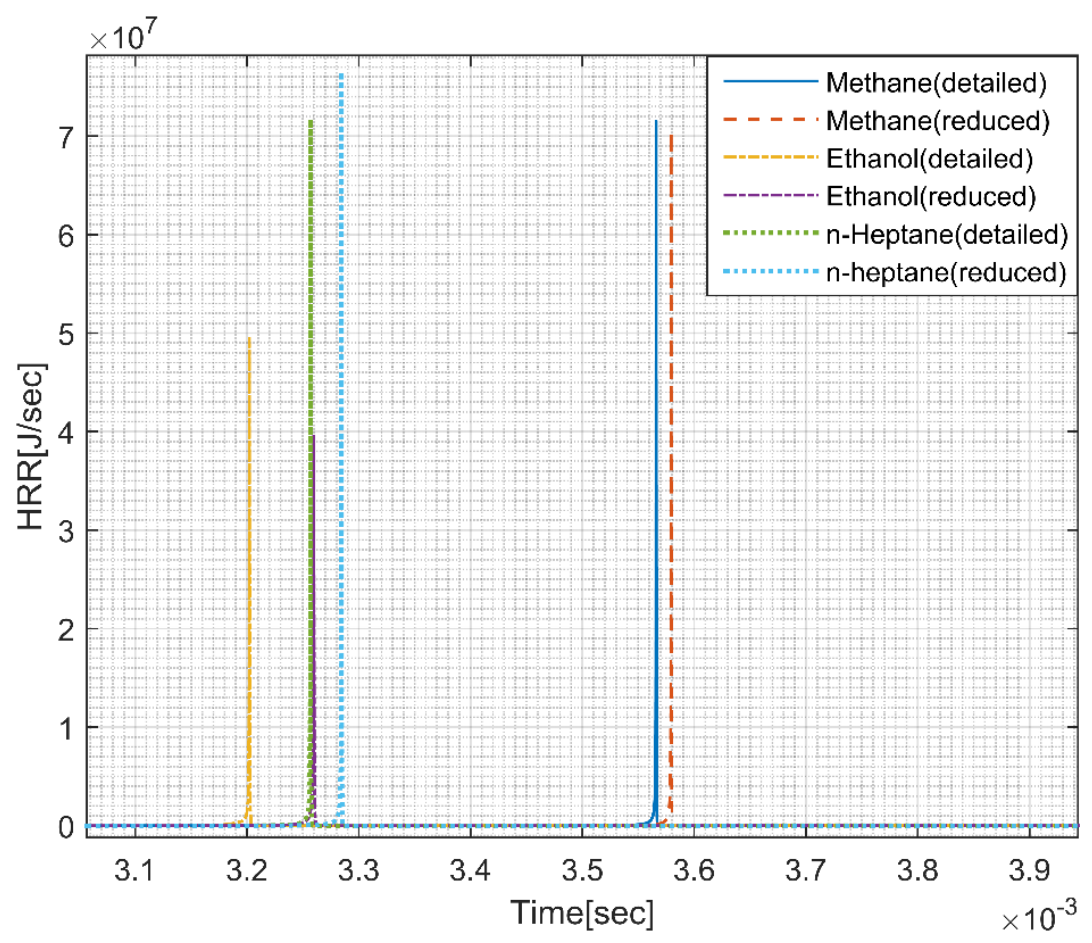

Figure 90 HRR for detailed and reduced mechanisms.

Figure 91 explains the effect of the mechanism reduction on the in-cylinder pressure. The pressure profiles are typically similar for each fuel but the difference is in the ignition pressure (ignition delay) and the peak pressure value. The variations in the ignition pressure can be explained by the same reasons, which were mentioned before to explain the variations in the ignition delay. The reduction process of some species and their reactions will change the ignition time and HRR values; therefore, the combustion pressure will vary as a result. In addition, the peak pressure differs as the detailed mechanism replaced by the reduced one. The difference in the peak pressure seems to be strongly coupled with the reduction steps, the difference is low for Methane and relatively high for $n$-heptane. 


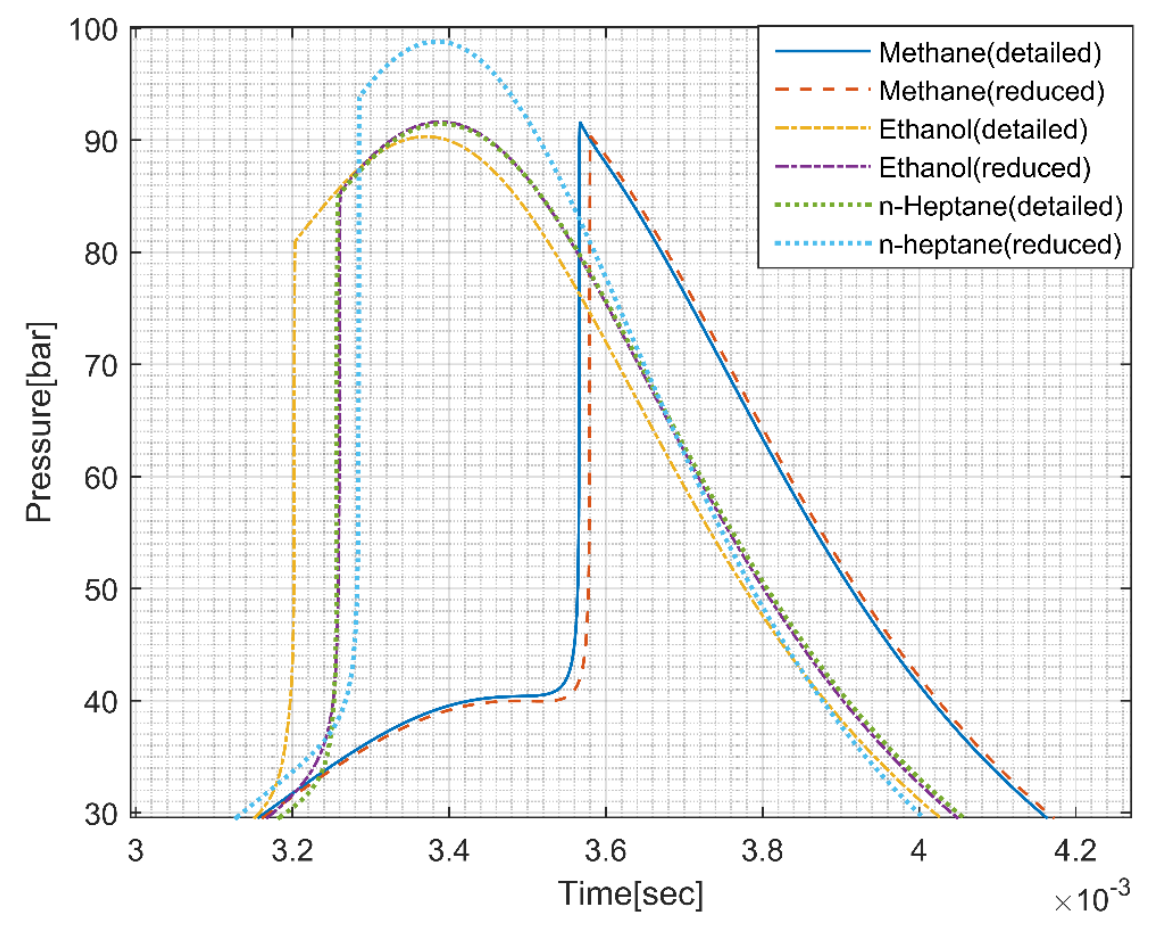

Figure 91 In-cylinder pressure (detailed vs. reduced mechanisms).

In Figure 92, the in-cylinder pressure is presented as a function of the piston position. The ignition location is clearly figured for each fuel mechanism (reduced \& detailed). Variations in the TDC locations are presented for each fuel, some fuels ignite after the TDC point while other ignite at the TDC. The mechanism reduction affects the location of TDC by changing the location of the TDC. Fuels that ignite at TDC have a closer TDC to the cylinder head when reduced mechanisms are applied. Typically, Figure 92 indicates less clearance volume for fuels that are represented by reduced mechanisms but with low knock resistance. However, this fact is not valid with high knock resistance fuels like Methane where the combustion occurs after the TDC location. Another observation is the significant difference between the TDC locations in the case of Ethanol and n-heptane while this difference is small for Methane. Such observation leads to the conclusion that the reduction steps and the nature of the reduced reactions have a strong effect on the ignition process. The Adverse work seems to be affected by the mechanism reduction as well. This work is less in the case of the reduced mechanisms for those fuels with short ignition delay. The earlier ignition events assist the increasing of the in-cylinder pressure since it will happen during the compression stroke. The increasing pressure will be met with slower expansion stroke, which will leave the piston with higher kinetic energy.

Next, the variations in the compression ratio due the mechanism reduction is illustrated in Figure 93. The compression ratio is almost the same for the detailed and the reduced mechanisms expect for $n$ heptane where the reduced mechanism results with higher compression ratio. The large number of reduction steps done on $n$-heptane mechanism is the reason for this noticeable difference in the compression ratio. 


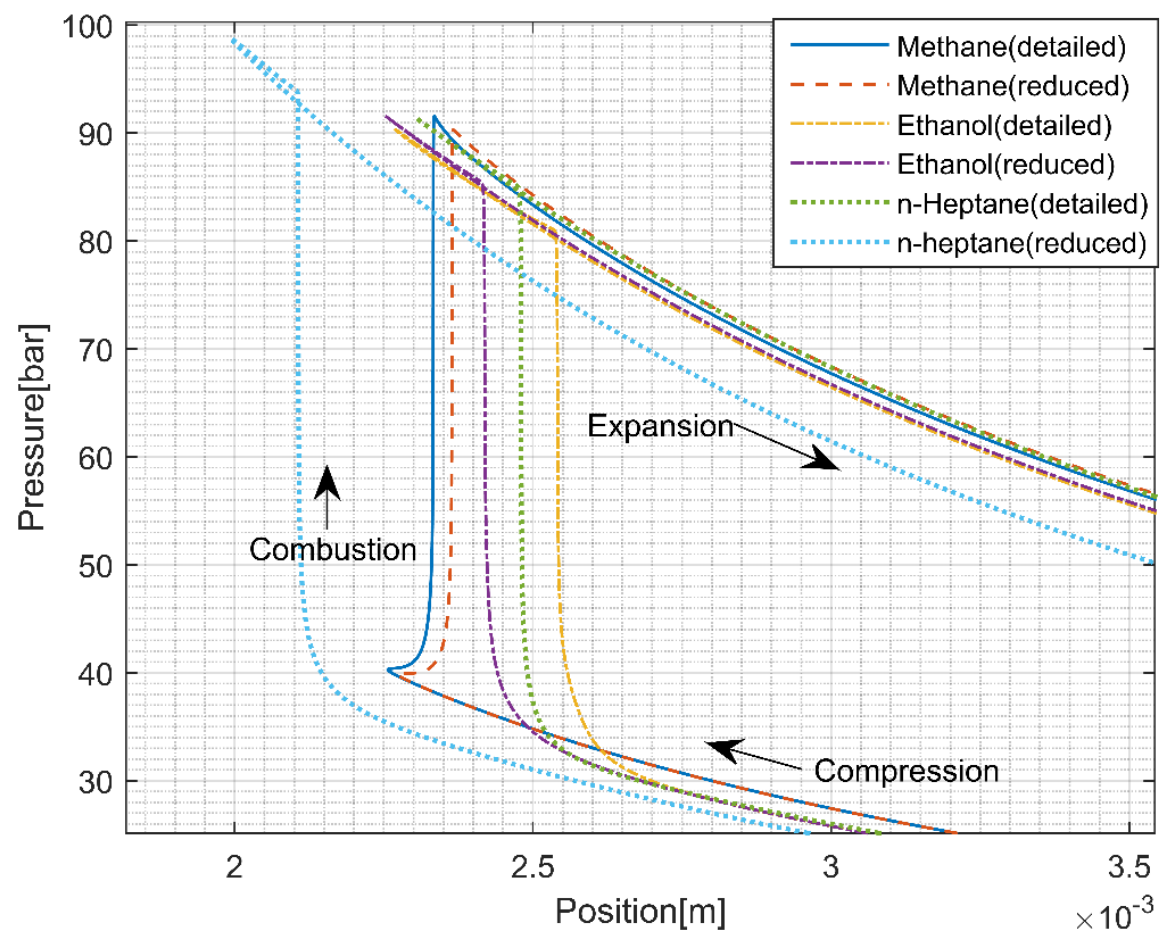

Figure 92 Pressure-position diagram (detailed vs. reduced mechanisms).

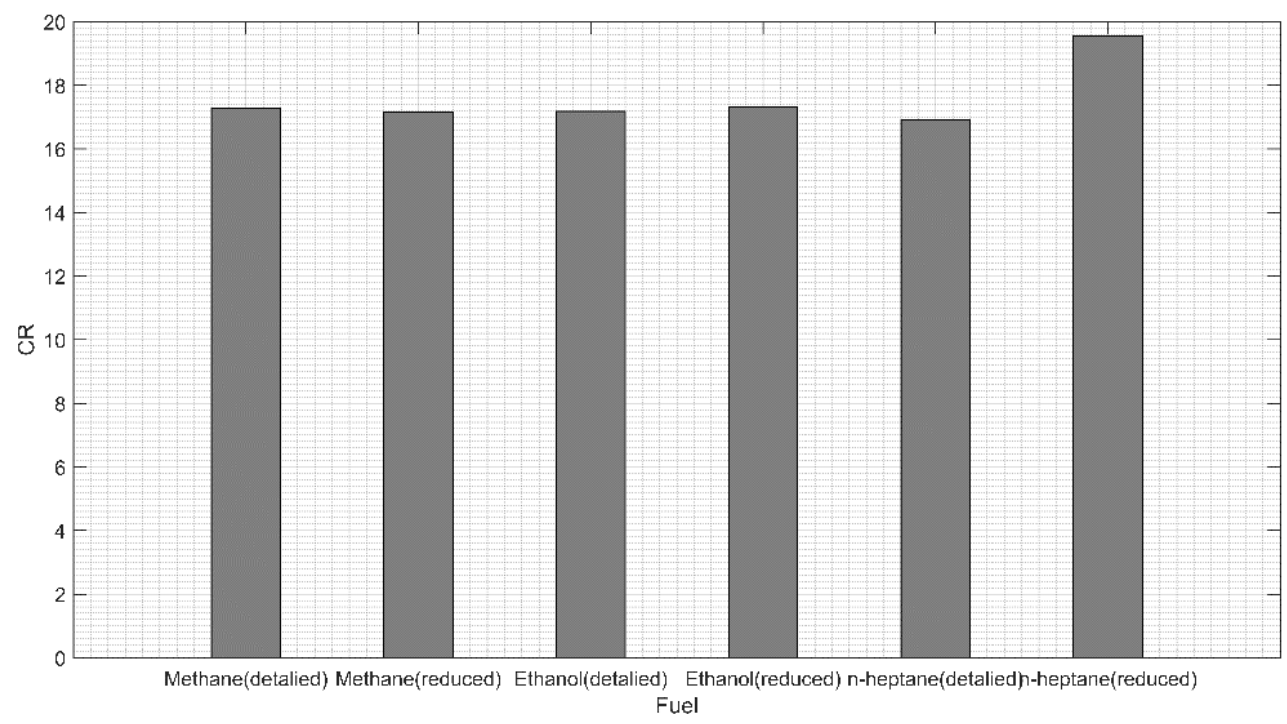

Figure 93 Compression ratio (detailed vs. reduced mechanism). 
As another comparison, the in-cylinder temperature variations is demonstrated in Figure 94 in order to investigate the effect of the mechanism reduction on the in cylinder temperature. The variations in the ignition delay are translated to variations in the self-ignition and the peak temperatures. In the case of the self-ignition temperature, Figure 94 indicates a slight difference in this temperature between the values presented by the detailed and the reduced mechanisms. In addition, the peak temperatures vary between the detailed and the reduced mechanism in more aggressive manner. This variation is slight for Methane and Ethanol fuels while it is significant for $n$-heptane. Such difference in the peak temperature can be explained by the difference in the HHR. The reduction process will affect many of the exothermic reaction that will assist increasing the HRR. Temperature investigations can be useful in estimation the $\mathrm{NO}_{\mathrm{x}}$ emissions since high peak temperatures refer usually to high $\mathrm{NO}_{x}$ formations. In fact, this section has been introduced to show the effect of the mechanism reduction on the free-piston performance specifically. However, many mechanisms are designed for emissions examinations by introducing extra reactions to represent the emissions formation.

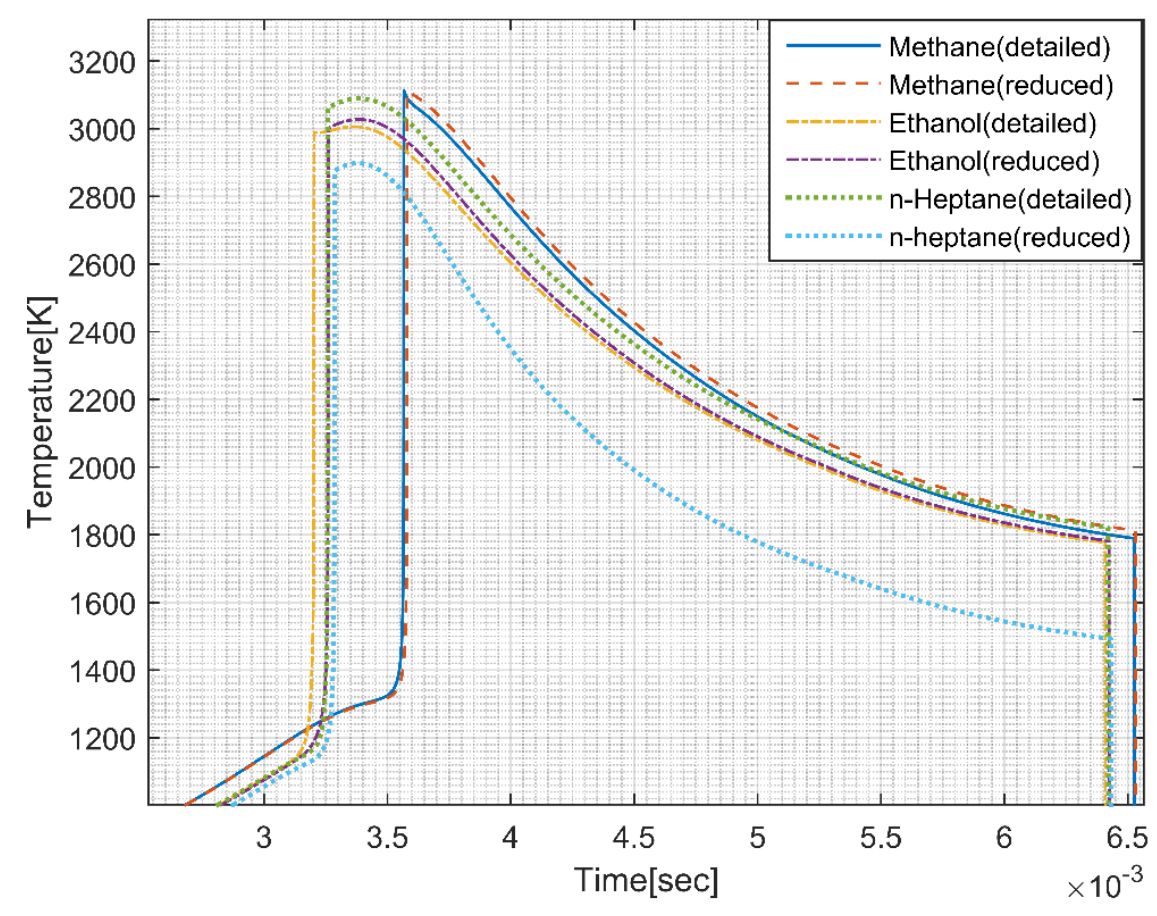

Figure 94 In-cylinder temperature (detailed vs. reduced). 
Lastly, the effect of the mechanism reduction on the overall work output ratio has been investigated as in Figure 95. The figure indicates that there is a slight difference in the overall work output ratio between the detailed and the reduced mechanisms. Such observations show the ability of the reduced mechanisms to replace the detailed ones with less computational costs. In general, mechanisms reduction is a good tool to cut the calculation time and investigate specific parameters within the engine system.

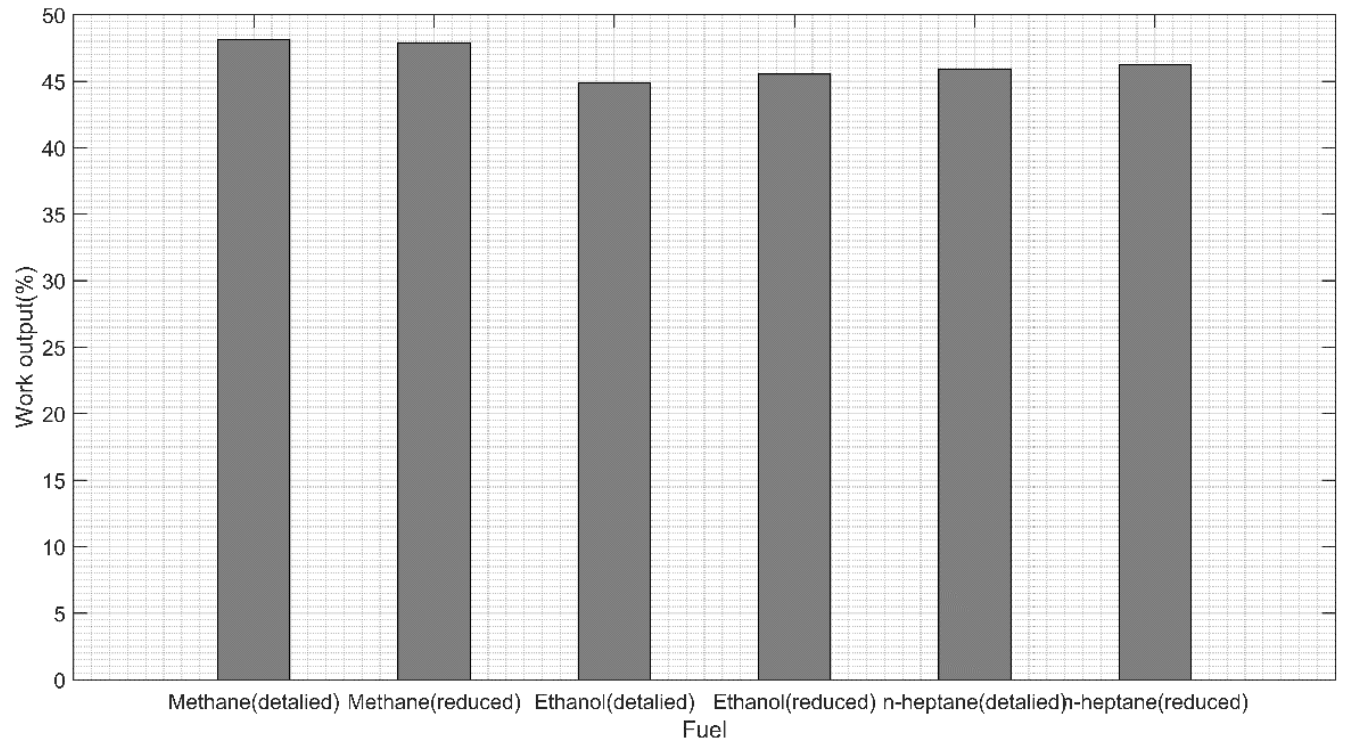

Figure 95 Work output ratio (detailed vs. reduced). 


\section{Chapter 8. Summary and conclusions}

Free-piston engines show their ability to replace the conventional slider-crank engines. The simple design and the high efficiency are among the features of the free-piston devices. Such features motivate the series of the researches and developments that have been introduced in this dissertation. Many topics have been covered within the dissertation body, starting with introducing the chemical kinetics approach, and ending with building a numerical model and examining its parameters.

The contributions of the dissertation can be summarized in the following points:

- Introducing the chemical kinetics approach in the free-piston engine field as a more accurate tool than the empirical approach in predicting the HRR.

- Study effect of the HRR on the piston motion, which assists in enhancing the overall system by understanding the relation between the dynamics and the thermodynamics of the free-piston engine.

- Study the effect of the different combustion phasing parameters on the system performance by studying parameters like the EGR, equivalence ratio and the intake temperature.

- Study the possibility of introducing a proper controlling technique that is able to drive the engine operation and eliminate the cyclic variations.

- Evaluating the model performance from emissions perception by considering main engine emissions like NOx and CO.

By introducing the chemical kinetics approach, the ability of truly examining the start of combustion and the HRR is more realistic than using the empirical approaches. In addition to their accuracy, chemical kinetics offers many ways to investigate the engine performance. Testing different fuels and fuel compositions, in addition to examining the system emissions are examples of the chemical kinetics abilities.

The contents of the dissertation cover three main parts, model building and simulation, model parameters examination and the model control. The basic model consists of two main sub-models, the dynamic sub-model and the in-cylinder thermodynamic one. In the case of the dynamic sub-model, a mathematical model has been presented based on Newton's second law by balancing the forces over the engine piston. The combustion pressure force demonstrates the gate between the two sub-models by connecting the fuel chemistry and the piston motion. The fuel-air mixture reactions have been calculated to obtain the HRR and therefore the in-cylinder pressure profile. Cantera toolbox presents the chemistry calculations in the study, in addition to $\mathrm{MATLAB}^{\circledR}$ as the main platform for all calculations.

The model describes fuel combustion in a single cylinder, two stroke free-piston engine under the $\mathrm{HCCl}$ combustion conditions. The system includes springs to support high frequencies and assist the piston during the compression stroke to avoid misfiring events. A single zone, zero-dimensional approach has been applied to simulate the fuel-air burning in PSR reactor that ensures a perfect and ideal gas mixing. In this approach, the fuel is assumed to be ignited homogeneously due to the increasing in its temperature and pressure solely, which simply presents the $\mathrm{HCCl}$ ignition mode. The nature of the fuel-air species reactions defines the whole aspects of the $\mathrm{HCCl}$ combustion. Cantera represents those reactions by analyzing the fuel mechanism and feeding the main code with the reactions rate. The species reaction equations, the first law of thermodynamics and the ideal gas law are solved as a system using stiff ODE solver. The resulting pressure and temperature data feed the dynamic sub-model in a cyclic manner until a certain tolerance is satisfied. The same simulation procedure is used when cases like EGR and 
equivalence ratio are applied. To demonstrate the engine operation, a multi-cycle simulation has been performed using different controlling techniques that consider the $\mathrm{HCCl}$ operation. The overall system efficiency has been evaluated based on the net delivered work to the electrical alternator.

The results of the simulations have been distributed along the dissertation chapters. At first, a multicycle simulation has been used to simulate the engine operation. Thereafter, the examining the effect of the system parameters on the overall performance has been done. Finally, investigations of the main engine emissions have been performed.

In the case of the multi-cycle simulation, the results describe the operation of the free-piston engine by simulating 100 cycles under the stoichiometric conditions. The cycles have an average frequency of $125 \mathrm{~Hz}$ at near-steady state conditions. The resulting electrical alternator converts $47 \%$ of the total chemical energy to electrical work. The stability of the engine strongly depends on the alternator load; this load is the core of the engine control scheme. It has been shown that $\mathrm{HCCl}$ ignition strongly affects the performance of the engine. Early ignition (just before TDC) events encourages better engine stability and performance. The transition from cycle to cycle is achieved with the use of a PD controller unit which adjust the alternator load based on the cyclic change in the piston bottom dead center position. The PD controller shows some limitations in performing a full steady state operation, however; it has the potential to guide the operation to desired number of cycles. Another try to push the model toward the steady state operation has been applied under the influence of a PI controller. The PI controller aims to reach a certain compression ratio by applying an alternator load, which is proportional to the piston velocity profile. The results of the PI controller indicate some unique performance after 15 cycles of operation where the engine starts to work between two compression ratios in a steady manner. The complexity of defining the exact relation between the fuel chemistry and the piston motion restricts the full understating of $\mathrm{PI}$ controller contribution.

At the same time, a study of many system parameters has been performed. One of the basic studies is to evaluate the effect of using some of the exhaust gases as one of the combustion reactants. The EGR impact on the combustion process and the formation of $\mathrm{NO}_{x}$ has been simulated and examined. The simulation results indicate an increase in the produced alternator work as the amount of EGR reach 30\% of the total charge mixture. The increasing in the compression stroke length and therefore the increasing in the in-cylinder pressure are the main explanations for the increase in the alternator work. The application of EGR shows the ability to significantly decrease the bulk peak temperature and therefore reduce the formation of the $\mathrm{NO}_{\mathrm{x}}$ emissions. In the other side, EGR decrease both of $\mathrm{CO}$ and $\mathrm{UHC}$ emissions, mainly methane molecules due to the low available temperatures for $\mathrm{CO}_{2}$ decomposition. The impact of EGR on the combustion and emissions is due to the reduced fuel- $\mathrm{O}_{2}$ concentrations within the reactants and the thermal and dilution effects of the $\mathrm{CO}_{2}$ and $\mathrm{H}_{2} \mathrm{O}$ molecules that found in EGR gases. Hot and cooled EGR charges have been applied as well; the two charges differ noticeably in their effect on the system parameters but not significantly in their effect on engine emissions. The nature of the emissions reactions depends potentially on the mixture composition rather than the mixture bulk temperature.

In addition to EGR, the effect of the intake temperature and the equivalence ratio have been examined. By evaluating the intake temperature effect, the results indicate that, the ignition event starts to happen earlier (before TDC) as the intake temperature increases. The HRR increases within the low and the intermediate temperature values of the applied temperatures range. A drop in the HHR is figured after that as relatively high intake temperatures are reached. The adverse work within the cycle is also affected 
by the intake temperature; it is observed that the area of this work is growing as the charge temperature increases. The net delivered work to the alternator increases as the intake temperature increases but not at high temperature values. It is shown that moderate intake temperatures have the optimal work output ratio since the combustion process is occurring at high compression ratios. In the emissions side, it is noticed that, the intake temperature has no effect on the emissions formation. In fact, the intake temperature accelerates the formation process, but it has no effect on the emissions quantities. Typically, emissions production is much controlled by the mixture composition than the mixture temperature where the nature of the involved species is the dominance.

Fuel-air equivalence ratio has been also examined within the dissertation; the impact of using rich and lean mixtures on the system performance is the target of this examination. It is found that, at constant intake temperature (540K); the HRR is less for lean mixtures $(\phi<1)$ than the rich mixtures $(\phi>1)$, while the in-cylinder peak pressure is higher for the lean mixtures. The peak temperature that is obtained when lean mixtures is burning is relatively low when compared with the one at rich or stoichiometric combustion. The difference in the ignition time and the HRR explain the previous observations. The effect of the equivalence ratio on the alternator work is found to be aggressive. Lean mixtures show much higher work output ratios than the rich ones in terms of the total fuel energy input. Emissions have been evaluated under this task as well, $\mathrm{NO}_{x}$ is found to be high in lean charges combustion due to the excessive $\mathrm{O}_{2}$ concentrations and the relatively high bulk temperatures. However, $\mathrm{CO}$ and $\mathrm{UHC}$ are much less for lean combustion since the possibility of having incomplete combustion is almost not available due to the plenty of air in those mixtures. For more examination, the intake temperature has been considered as a second factor beside the equivalence ratio. Relatively cold charges with lean properties show the most efficient performance as long as the lowest peak temperatures. In the other side, rich mixtures with moderate intake temperature values show in-cylinder pressure levels similar to those found in lean combustion process. In terms of emissions, no effect is obtained for the different intake temperature values for both of the rich and lean combustion.

The dissertation includes a specific study of the ultra-lean mixtures as well. The aim of including the ultra-lean concept is to show the ability of the lean mixtures in reducing $\mathrm{NO}_{\mathrm{x}}$ emissions. The results indicate $\mathrm{NO}_{\mathrm{x}}$ levels as low as those levels found in the case of the rich mixtures burning. However, the alternator work output starts to drop while the mixture goes to extreme lean values. The main reason behind the ability of the ultra-lean mixture to reduce $\mathrm{NO}_{x}$ formation is the great drop in the combustion peak temperatures. The dilution effects done by the excessive air molecules in the combustion chamber explain such drop in the bulk temperature. In the other side, the power output is reduced because of the less introduced fuel into the cylinder and also due to the poor combustion efficiency which cause an expected unburned fuel in the exhaust products.

Finally, the fuel type and the fuel mechanism have been investigated to obtain the sensitivity of the engine model to those factors. When different fuels have been tested, the variation of the performance seems obviously. Fuels like DME ignite early where fuels like Methane take longer time. The unrestricted nature of the free-piston engine allows the full comparison between the different fuels. In general, the model shows the ability of the engine to work with different fuels. In the case of the fuel mechanism, the results indicate close performance between the full and reduced mechanisms while some differences are observed in some parameters like the mixture pressure and temperature. 


\section{Chapter 9. References}

[1] M. Prados, Towards a Linear Engine, PhD Dissertation, Stanford, CA, 2002.

[2] G. Shaver, "Physics-Based Modeling and Control of Residual-Affected HCCl Engines Using Variable Valve Actuation," PhD Dissertation, Stanford, CA, 2005.

[3] A. Charalambides, "Homogeneous Charge Compression Ignition (HCCl) Engines," in Advances in Internal Combustion Engines and Fuel Technologies, 2013.

[4] H. Bendu and M. S. , "Homogeneous Charge Compression Ignition (HCCl) Combustion: Mixture Preparation and Control Strategies in Diesel Engines," Renewable and Sustainable Energy Reviews, vol. 38, pp. 732-746, 2014.

[5] J. Griffiths and J. Barnard, Flame and Combustion, CRC Press, Inc, 1995.

[6] J. Heywood, Internal Combustion Engine Fundamentals, McGraw-Hill Education, 1988.

[7] R. Stone, Introduction to Internal Combustion Engines, 1992.

[8] Z. Peng, H. Zhao and N. Ladommatos, "Visualization of the homogeneous charge compression ignition/controlled autoignition combustion process using two dimensional planar laser-induced fluorescence image of formaldehyde," Proc Instn Mech Engrs, no. 217, pp. 1125-1134, 2003.

[9] G. Aleiferis, G. Charalambides, Y. Hardalupas, P. Taylor and Y. Urata, "Modelling and Experiments of HCCI Engine Combustion with Charge Stratification and Internal EGR," SAE paper, no. 2005-013725, 2005.

[10] A. Hultqvist, M. Christensen, B. Johansson, M. Richter, J. Nygren, J. Hult and M. Alden, "The HCCI Combustion Process in a Single Cycle- Speed Fuel Tracer LIF and hemi-luminescence Imaging," SAE Paper, no. 2002-01-0424, 2002.

[11] D. Bradley, C. Morley, J. Gu and R. Emerson, "Amplified pressure waves during autoignition: relevance to CAI engines," SAE paper, no. 2002-01-2868, 2002.

[12] R. Tominaga, S. Morimoto, Y. Kawabata, S. Matsuo and T. Amano, "Effects of heterogeneous EGR on the natural gas fueled $\mathrm{HCCl}$ engine using experiments," CFD and detailed kinetics, SAE paper, no. 2004-01-0945, 2004.

[13] J. Hyvönen, G. Haraldsson and B. Johansson, "Supercharging $\mathrm{HCCl}$ to Extend the Operating Range in a Multi-Cylinder VCR-HCCI Engine," SAE paper, no. 2003-01-3214, 2003.

[14] R. Pescara, "Motor compressor apparatus," US Patent 1,657,641, 1928.

[15] H. Farmer, "Free-piston compressor-engines," Proceedings of the Institution of Mechanical Engineers: Automobile Division, vol. 156, pp. 253-271., 1947. 
[16] T. Callahan and S. Ingram, "Free Piston Engine Linear Generator for Hybrid vehicles Modeling Study," Center for Electro-mechanics at The University of Texas, US Army, Austin, TX, 1995.

[17] P. Van Blarigan, N. Paradiso and S. Goldsborough, "Homogeneous Charge Compression Ignition with a Free Piston; A New Approach to Ideal Otto Cycle Performance," 1998.

[18] S. Goldsborough and P. Van Blarigan, "A Numerical Study of a Free Piston IC Engine Operating on Homogeneous Charge Compression Ignition Combustion," SAE paper, 1999.

[19] P. Van Blarigan, "Advanced Internal Combustion Electrical Generator," in in Proceedings of the 2001 DOE Hydrogen Program Review, Livermore, CA, 2001.

[20] S. Goldsborough and P. Van Blarigan, "Optimizing the Scavenging System for a Two-Stroke Cycle, Free Piston Engine for High Efficiency and Low Emissions: A Computational Approach," SAE paper, no. 2003-01-0001, 2003.

[21] P. Koning, "Free-Piston Engines: A Possible Route to Hybrid-Electric Vehicles," 26 March 2014. [Online]. Available: http://crf.sandia.gov/free-piston-engines-a-possible-route-to-hybrid-electricvehicle/.

[22] N. Clark, S. Nandkumar, C. Atkinson, R. Atkinson and T. P. S. McDaniel, "Experimental Examination of a Small Bore Linear Two-Stroke Engine," in ASME Internal Combustion Engine Fall Conference, Clymer, NY, pp. 33-42, 1998.

[23] C. Atkinson, S. Petreanu, C. Clark, R. Atkinson, T. McDaniel and S. Nandkumar, "Numerical Simulation of a Two-Stroke Linear Engine-Alternator Combination," SAE paper, no. 1999-01-0921, 1999.

[24] E. Shoukry, S. Taylor, N. Clark and P. Famouri, "Numerical Simulation for Parametric Study of a TwoStroke Direct Injection Linear Engine," SAE paper, no. 2002-01-1739, 2002.

[25] C. Tóth-Nagy, Linear Engine Development for Series Hybrid Electric Vehicles, Ph.D. Dissertation, West Virginia University, 2004.

[26] R. Mikalsen and A. Roskilly, "A Review of Free-Piston Engine History and Applications," Applied Thermal Engineering, vol. 27, pp. 2339-2352, 2007.

[27] R. Mikalsen and A. Roskilly, "Performance Simulation of a Spark Ignited Free-Piston Engine GeneratoR," Applied Thermal Engineering, vol. 28, pp. 1726-1733, 2008.

[28] R. Mikalsen and A. Roskilly, "The Design and Simulation of a Two-Stroke Free-Piston ompression Ignition Engine for Electrical Power Generation," Applied Thermal Engineering, vol. 28, pp. 589600, 2008.

[29] R. Mikalsen and A. Roskilly, "A Computational Study of Free-Piston Diesel Engine Combustion," Applied Energy, vol. 86, pp. 1136-1143, 2009. 
[30] R. Mikalsen and A. Roskilly, "The Control of a Free-Piston Engine Generator. Part 1: Fundamental Analyses," Applied Energy, vol. 87, pp. 1273-1280, 2010.

[31] R. Mikalsen and A. Roskilly, "The Control of a Free-Piston Engine Generator. Part 2: Engine Dynamics and Piston Motion Control," Applied Energy, vol. 87, no. 4, pp. 1281-1287, 2010.

[32] M. Hanipah, R. Mikalsen and A. Roskilly, "Recent Commercial Free-Piston Engine Developments for Automotive Applications," Applied Thermal Engineering, vol. 75, pp. 493-503, 2015.

[33] J. Fredriksson and I. Denbratt, "Simulation of a Two-Stroke Free Piston Engine," SAE paper, no. 2004-01-1871, 2004.

[34] D. Carter and E. Wechner, "The Free Piston Power Pack: Sustainable Power for Hybrid Electric Vehicles," SAE paper, no. 2003-01-3277, 2003.

[35] A. Kleemann, J. Dabadie and S. Henriot, "Computational Design Studies for a High Efficiency and Low-Emissions Free Piston Engine Prototype," SAE paper, no. 200401-2928, 2004.

[36] S. Ashley, "Free-Piston IC Generator Developed for Range-Extender Hybrids," Automotive Engineering Magazine, 2013. [Online]. Available: http://articles.sae.org/12284/.

[37] H. Kosaka, K. Akita, K. Moriya, S. Goto, Y. Hotta and T. Umeno, "Development of Free Piston Engine Linear Generator System Part 1 - Investigation of Fundamental Characteristics," SAE paper, no. 2014-01-1203, 2014.

[38] S. Goto, K. Moriya, H. Kosaka, T. Akita, Y. Hotta and T. Umeno, "Development of Free Piston Engine Linear Generator System Part 2- Investigation of Control System for Generator," SAE paper, no. 2014-01-1193, 2014.

[39] N. Hung, O. Lim and N. lida, "The Effects of Key Parameters on the Transition from SI Combustion to $\mathrm{HCCl}$ Combustion in a Two-Stroke Free Piston Linear Engine," Applied Energy, vol. 137, pp. 385401, 2015.

[40] M. Robinson and N. Clark, "Fundamental Analysis of Spring-Varied, Free Piston, Otto Engine Device," SAE International Journal of Engines, vol. 7, 2014.

[41] M. Robinson and N. Clark, "Fundamental Explorations of Spring-Varied, Free Piston Linear Engine Devices," Journal of Engineering for Gas Turbines and Power, vol. 137, 2015.

[42] S. Aceves, D. Flowers, F. Loza and J. Frias, "Piston-Liner Crevice Geometry Effect on $\mathrm{HCCl}$ Combustion by Multi-Zone Analysis," SAE paper, no. 2002-01-2869, 2002.

[43] Y. Wong and G. Karim, "A kinetic examination of the effects of recycled exhaust gases on the autoignition of homogeneous n-heptane-air mixtures in engines," SAE paper, no. 2000-01-2037, 2000. 
[44] Y. Yamasaki and N. lida, "Numerical simulation of auto-ignition and combustion of $\mathrm{n}$-butane and air mixtures in a 4-stroke $\mathrm{HCCl}$ engine by using elementary reactions," SAE paper, no. 2000-01$1834,2000$.

[45] S. Fiveland and D. Assanis, "Development and validation of a quasi-dimensional model for $\mathrm{HCCl}$ engine performance and emissions studies under turbocharged conditions," SAE paper, no. 200201-1757, 2002.

[46] W. Easley, A. Agarwal and G. Lavoie, "Modeling of $\mathrm{HCCl}$ combustion and emissions using detailed chemistry," SAE paper, no. 2001-01-1029, 2001.

[47] T. Noda and D. Foster, "A numerical study to control combustion duration of hydrogen-fueled $\mathrm{HCCl}$ by using multi-zone chemical kinetics simulation," SAE paper, no. 2001-01-0250, 2001.

[48] A. Babajimopoulos, D. Assanis and S. Fiveland, "Modeling the effects of gas exchange processes on $\mathrm{HCCl}$ combustion and an evaluation of potential control through variable valve actuation," SAE paper, no. 2002-01-2829, 2002.

[49] J. Kusaka, Y. Mandokoro, S. Kubo and S. Yamazaki, "Predicting homogeneous charge compression ignition characteristics of various hydrocarbons," in Proceedings of the 15th Internal Combustion Engine Symposium (International), Seoul, Korea, 1999.

[50] P. Najt and D. Foster, "Compression-Ignited Homogeneous Charge Combustion," SAE paper, no. 830264, 1983, doi:10.4271/830264, 1983.

[51] Q. Li, J. Xiao and Z. Huang, "Simulation of a Two-Stroke Free-Piston Engine for Electrical Power Generation," Energy and Fuels, vol. 22, no. 5, pp. 3443-3449, 2008.

[52] C. Zhang, K. Li and Z. Sun, "Modeling of Piston Trajectory-Based HCCl Combustion Enabled by a Free Piston Engine," Applied Energy, vol. 139, pp. 313-326, 2015.

[53] F. Taylor and T. Toong, "Heat Transfer in Internal-Combustion Engines," American Society of Mechanical Engineers, no. 57-HT-17, 1957.

[54] W. Annand, "Heat Transfer in the Cylinder and Porting," The Thermodynamics and Gas Dynamics of Internal Combustion Engines, vol. 2., 1986.

[55] W. Annand, "Heat Transfer in the Cylinders of Reciprocating Internal Combustion Engines," Proc. Instn. Mech. Engrs, vol. 177, no. 36, pp. 973-990, 1963.

[56] G. Eichelberg, "Some Investigations on Old Combustion-Engine Problems," Engineering, London, vol. 148, no. 1, 2, pp. 463-466, 547-550, 1939.

[57] G. Woschni, "A Universally Applicable Equation for the Instantaneous Heat Transfer Coefficient in the Internal Combustion engine," SAE Paper, no. 670931, 1967. 
[58] T. LeFeuvre, P. Myers and O. Uyehara, "Experimental Instantaneous Heat Fluxes in a Diesel Engine and Their Correlation," SAE paper, vol. 78, no. 690464, 1969.

[59] J. Dent and S. Suliaman, "Convective and Radiative Heat Transfer in a HighSwirl Direct injection Diesel Engine," SAE paper, vol. 86, no. 770407, 1977.

[60] F. Agrell, H. Angstrom, B. Eriksson, J. Wikander and J. Linderyd, "Transient control of HCCl through combined intake and exhaust valve actuation," SAE paper, no. 2003-01-3172, 2003.

[61] G. Haraldsson, P. Tunestal, B. Johansson and J. Hyvonen, "HCCl combustion phasing with closedloop combustion control using variable compression ratio in a multi cylinder engine," JSAE, no. 20030126, 2003.

[62] J. Olsson, P. Tunestal and B. Johansson, "Closed-loop control of an $\mathrm{HCCl}$ engine," SAE paper, no. 2001-01-1031, 2001.

[63] R. Flores, M. Miyasato, V. McDonell and G. Samuelsen, "Response of a Model Gas Turbine Combustor to Variation in Gaseous Fuel Composition," 2001.

[64] E. Peterson, J. Hall, S. Smith, J. de Vries, A. Amadio and M. Crofton, "Ignition of Lean MethaneBased Fuel Blends at Gas Turbine Pressures," ASME J. Eng. Gas Turbines Power, vol. 129, pp. 937944, 2007.

[65] W. Liss, W. Thrasher, G. Steinmetz, P. Chowdiah and A. Attari, "Variability of Natural Gas Composition in Select Major Metropolitan Areas of the United States," 1992.

[66] L. Spadaccini and M. Colket, "Ignition Delay Characteristics of Methane Fuels," Prog. Energy Combust. Sci, vol. 20, pp. 431-460, 1994.

[67] V. Duggal, "Facsimile form Vinod Duggal, Cummins Engine Co, to Lesley Crowell, ARB," February 26, 2001.

[68] K. Spletter and L. Adair, "US gas-processing profitability statistics begin in this issue", Oil and Gas Journal, 99(21), pp. 542001.

[69] "United States Department of Energy," 2013. [Online]. Available: http://energy.gov/.

[70] P. Anderson, "Algorithm for Methane Number Determination for Natural Gasses," Danish Gas Technology Centre, 1999.

[71] M. Lapuerta, J. Hernandez and F. Gimenez, "Evaluation of exhaust gas recirculation as a technique for reducing Diesel engine NOx emissions," Proc Instn Mech Engrs Part D, J Autom Engng, p. 8593., 2000.

[72] H. Machacon, S. Shiga, T. Karasawa and H. Nakamura, "Simultaneous reduction of soot and NOx by intake gas variation," 6th International Symposium on Marine Engineering, 2000. 
[73] T. Murayama, M. Zheng, T. Chikahisa, Y. Oh, Y. Fujiwara, S. Tosaka and e. al., "Murayama T, Zheng M, Chikahisa T, Oh Y, Simultaneous reductions of smoke and NOx from a DI Diesel engine with EGR and dimethyl carbonate.," SAE Transactions., no. 952518., 1995.

[74] D. Tomazic and A. Pfeifer, "Tomazic D, Pfeifer A. Cooled EGR—a must or an option for 2002/04.," SAE paper, no. 2002-01-0962, 2002.

[75] P. Duret, B. Gatellier, L. Monteiro, M. Miche, P. Zima and D. Maroteaux, "Progress in diesel HCCI combustion within the European SPACE LIGHT project," SAE paper, no. 2004-01-1904, 2004.

[76] M. Sjoberg and J. Dec, "An investigation of the relationship between measured intake temperature, $\mathrm{BDC}$ temperature, and combustion phasing for premixed and $\mathrm{DI} \mathrm{HCCl}$ engines," SAE paper, no. 2004-01-1900, 2004.

[77] G. Hampson, "Heat release design method for $\mathrm{HCCl}$ in diesel engines," SAE paper, no. 2005-01$3728,2005$.

[78] V. Hosseini and M. Checkel, "Using reformer gas to enhance $\mathrm{HCCl}$ combustion of NG in a CFR engine," SAE paper, no. 2006-01-3247, 2006.

[79] S. Gan, H. Ng and K. Pang, "Homogeneous charge compression ignition (HCCl) combustion: implementation and effects on pollutants in direct injection diesel engines," Appl Energy, vol. 88, pp. 559-67, 2011.

[80] H. Zhao, Z. Peng, J. Williams and N. Ladommatos, "Understanding the effects of recycled burnt gases on the controlled autoignition (CAI) combustion in four-stroke gasoline engines," SAE paper, no. 2001-01-3607, 2001.

[81] H. Zhao, Z. Peng and N. Ladommatos, "Understanding of controlled autoignition combustion in a four-stroke gasoline engine," in Proc Inst Mech Eng Part D: J Autom Eng, 2001.

[82] H. Machrafi, S. Cavadias and P. Guibert, "An experimental and numerical investigation on the influence of external gas recirculation on the $\mathrm{HCCl}$ autoignition process in an engine: thermal, diluting, and chemical effects," Combust Flame, vol. 155, p. 476-489., 2008.

[83] Machrafi, S. Cavadias and J. Amouroux, "A parametric study on the emissions from an HCCI alternative combustion engine resulting from the auto-ignition of primary reference fuels," Appl Energy, vol. 85, pp. 755-64, 2008.

[84] Xutao. and Wangyang, "Simulation of a two-stroke dimethyl -ether free piston engine operating on $\mathrm{HCCl}$ combustion," in International Conference on Transportation, Mechanical, and Electrical Engineering (TMEE), Changchun, China, December 16-18,2011.

[85] C. Zhang, J. Pan, J. Tong and J. Li, "Effects of intake temperature and excessive air coefficient on combustion characteristics and emissions of $\mathrm{HCCl}$ combustion," 2nd International Conference on 
Challenges in Environmental Science and Computer, vol. Volume 11, no. PART C, pp. 1119-1127, 2011.

[86] R. Maurya and A. Agarwal, "Experimental study of combustion and emission characteristics of ethanol fuelled port injected homogeneous charge compression ignition ( $\mathrm{HCCl}$ ) combustion engine," Applied Energy, vol. 88, no. 4, pp. 1169-1180, 2011.

[87] M. Lida, M. Hayashi, D. Foster and J. Martin, "Characteristics of homogeneous charge compression ignition $(\mathrm{HCCl}$ ) engine operation for variations in compression ratio, speed, and intake temperature while using n-butane as a fuel," Journal of Engineering for Gas Turbines and Power, vol. 125, pp. 472-478, 2003.

[88] C. Cinar, A. Uyumaz, H. Solmaz, F. Sahin, S. Polat and E. Yilmaz, "Effects of intake air temperature on combustion, performance and emission characteristics of a $\mathrm{HCCl}$ engine fueled with the blends of $20 \%$ n-heptane and $80 \%$ isooctane fuels," Fuel Processing Technology, vol. 130, pp. 275-281, 2015.

[89] J. Ghafouri, S. Shafee and A. Maghbouli, "Investigation on effect of equivalence ratio and engine speed on homogeneous charge compression ignition combustion using chemistry based CFD code," Thermal Science, vol. 18, no. 1, pp. 89-96, 2014.

[90] A. Rahbari, "Effect of inlet temperature and equivalence ratio on $\mathrm{HCCl}$ engine performance fuelled with ethanol: Numerical investigation," Journal of Central South University, Springer, no. (2016) 23: 122-129, 2016.

[91] K. Hamada, K. Nijima and K. Yoshida, "The Effects of the Compression Ratio, Equivalence Ratio, and Intake Air Temperature on Ignition Timing in an HCCI Engine Using DME Fuel," SAE paper, no. 200532-0002, 2005.

[92] D. Rankin, LEAN COMBUSTION : Technology and Control, Academic Press, 2011.

[93] D. Rankin, LEAN COMBUSTION: Fundamentals, Applications, and Prospects, Irvine, California: Academic Press, 2007.

[94] M. Aliramezania, I. Chitsazb and A. Mozafari, "Thermodynamic modeling of partially stratified charge engine characteristics for hydrogen-methane blends at ultra-lean conditions," International Journal of Hydrogen Energy, vol. 38, no. 25, pp. 10640-10647, 2013.

[95] J. Greenwood, P. Erickson, J. Hwang and J. Jordan, "Experimental results of hydrogen enrichment of ethanol in an ultra-lean internal combustion engine," International Journal of Hydrogen Energy, vol. 39, no. 24, pp. 12980-12990, 2014.

[96] S. Kokjohn, "Reactivity Controlled Compression Ignition (RCCl) Combustion," PhD dissertation, University of Wisconsin-Madison, Madison, WI, 2012. 
[97] L. Olivier and L. Khaled, "Natural gas: physical properties and combustion features," www.intechopen.com, Nantes, Cedex 3, France, 2010.

[98] H. Wang, R. Reitz, M. Yao, B. Yang, Q. Jiao and L. Qiu, "Development of an n-heptane-n-butanolPAH mechanism and its application for combustion and soot prediction," Combustion and Flame, vol. 160, no. 3, pp. 504-519, 2013.

[99] C. Jin, M. Yao, H. Liu, C. Lee and J. Ji, "Progress in the production and application of $\mathrm{n}$-butanol as a biofuel," Renewable and Sustainable Energy Reviews, vol. 15, no. 8, pp. 4080-4106, 2011.

[100] C. Arcoumanis, C. Bae, R. Crookes and E. Kinoshita, "The potential of di-methyl ether (DME) as an alternative fuel for compression-ignition engines: A review," Fuel, vol. 87, no. 7, pp. 1014-1030, 2008.

[101] A. Boehman, "Developments in production and utilization of dimethyl ether for fuel applications," Fuel Processing Technology, vol. 89, no. 12, pp. 1243, 2008.

[102] T. Zeuch, G. Moréac, S. Ahmed and F. Mauss, "A comprehensive skeletal mechanism for the oxidation of n-heptane generated by chemistry-guided reduction," Combustion and Flame, vol. 155, no. 4, pp. 651-674, 2008.

[103] F. Williams, Combustion Theory, The Fundamental Theory of Chemically Reacting Systems 2nd ed, Menlo Park, CA : Benjamin Cummings Publishing Co, 1985.

[104] N. Peters, "Reducing Mechanisms, Reduced Kinetic Mechanisms and Asymptotic Approximations for Methane-Air Flames," in Springer-Verlag, Berlin-Heidelberg, 1991.

[105] C. Seigneur, G. Stephanopoulos and J. Carr, "Dynamic Sensitivity Analysis of Chemical Reaction Systems," Chem. Eng. Sci, vol. 17, no. 845, 1982.

[106] J. Carr, D. Peterson and F. Smith, "Flash Photolysis of 1,3-Dichlorotetrafluoroacetone in the Presence of Oxygen, Kinetics netics and Mechanism of the Oxidation of the Chlorodifluoromethyl Radicals," J. of Physical Chemistry, vol. 90, no. 697, 1986.

[107] O. Gautier and J. Carr, "Variational Sensitivity Analysis of a Photochemical Smog Mechanism," Int. J. of Chemical Kinetics, vol. 17, no. 1347, 1985.

[108] H. Kim, S. Cho and K. Min, "Reduced Chemical Kinetic Model of DME for HCCl Combustion," SAE papers, no. 2003-01-1822, 2003.

[109] S. Aceves, J. Frias, D. Flowers and J. J. Smith, "A Computer Generated Reduced Iso-Octane Chemical Kinetic Mechanism Applied to Simulation of HCCl Combustion," SAE papers, no. 2002-01-2870, 2002.

[110] R. Kee, F. Rupley, J. Miller, M. Coltrin, J. Grcar, E. Meeks, H. Moffat, A. Lutz, g. Dixon-Lewis, M. Smooke, J. Warnatz, G. Evans, R. Larson, R. Mitchell, L. Petzold, W. Reynolds, M. Caracotsios, W. 
Stewart, P. Glarborg, C. Wang, C. McLellan, O. Adigun, W. Houf, c. Chou, S. Miller, P. ho, P. Young and D. Young, "CHEMKIN Release 4.0.2," Reaction Design, San Diego, CA, 2005.

[111] J. Duterque, R. Borghi and H. Tichtinsky, "Study of Quasi-Global Schemes for Hydrocarbon Combustion," Combustion Science and Technology, vol. 26, no. 1-2, pp. 1-15, 1981.

[112] W. Jones and R. Lindstedt, "Global Reaction Schemes for Hydrocarbon Combustion," combustion and Flame, vol. 73, pp. 233-249, 1988.

[113] G. Smith, D. Golden, M. Frenklach, N. Moriarty, B. Eiteneer, M. Goldenberg, c. Bowman, R. Hanson, S. song, W. Gardiner, v. Lissianski and Z. Quin, "GRI-MECH 3.0".

[114] V. Ganesan, Internal combustion engines, McGraw-Hill, 2003.

[115] J. Chiang and A. Stefanopoulou, "Sensitivity Analysis of Combustion Timing and Duration of Homogeneous Charge Compression Ignition (HCCl) Engines," in Proceeding of the 2006 American Control Conference, 2006.

[116] K. Sigmon, MATLAB Primer, CRC Press, Inc, 2002.

[117] D. Goodwin, N. Malaya and R. Speth, "Cantera: an object-oriented software for chemical kinetics, thermodynamics and transport processes," [Online]. Available:

https://code.google.com/p/cantera/>.

[118] K. Swan, M. Shahbakhti and C. Koch, "Predicting Start of Combustion Using a Modified Knock Integral Method for an HCCI Engine," SAE 2006 Transactions Journal of Engines, pp. 611-620, 2007.

[119] E. Sher and R. Harari, "A Simple and Realistic Model for the Scavenging Process in a Crankcase Scavenged Two Stroke Cycle Engine," Proceedings of the Institution of Mechanical Engineers, vol. 205, pp. 129- 137, 1991.

[120] N. Clark, S. Nandkumar, C. Atkinson, R. Atkinson, T. McDaniel, S. Petreanu, P. Famouri and w. Cawthorne, "Operation of a Small Bore Two-Stroke Linear Engine," 1998.

[121] R. Kee, M. Coltrin and P. Glarborg, Chemically Reacting Flow: Theory and Practice, Wiley, 2003.

[122] R. Turns, An Introduction to Combustion, McGraw Hill, 2011.

[123] F. Yan and J. Wang, "Engine Cycle-by-Cycle Cylinder Wall Temperature Observer-Based Estimation through Cylinder Pressure Signals," Journal of Dynamic Systems, Measurements, and Control, vol. 134, no. 6, pp. 1-8, 2012.

[124] S. Schneider, F. Rinderknecht and H. Friedrich, "Design of Future Concepts and Variants of the Free Piston Linear Generator," in 9th International Conference in Ecological Vehicles and Renewable Energies, Monte-Carlo, France, 2014. 
[125] Z. Zhao, D. Wu, Z. Zhang, F. Zhang and C. Zhao, "Experimental Investigation of the Cycle-to-Cycle Variations in Combustion Process of a Hydraulic Free-Piston Engine," Energy, vol. 78, pp. 257-265, 2014.

[126] R. Maurya and A. Agarwal, "Experimental Investigation of Cycle-by-Cycle Variations in CAl/HCCl combustion of Gasoline and Methanol Fuelled Engine," 2009.

[127] K. Åström, Control System Design, University of California, 2002.

[128] W. May, "Reduction of Thermal and Prompt NOx In Exhausts of Natural Gas Fueled Boilers," SFA International Technical Article Library, 2012.

[129] F. Xie, X. Li, Y. Su, W. Hong, B. Jiang and L. Han, "Influence of air and EGR dilutions on improving performance of ahigh compression ratio spark-ignition engine fueled with methanol at light load," Applied Thermal Engineering, vol. 94, p. 559-567, 2016.

[130] H. Wei, T. Zhu, G. Shu, L. Tan and Y. Wang, "Gasoline engine exhaust gas recirculation - A review," Applied Energy, vol. 99, pp. 534-544, 2012.

[131] Y. Levendis, I. Pavlatos and R. Abrams, "Control of diesel soot, hydrocarbon and NOx emission with a particulate trap and EGR," SAE paper, no. 940460, 1994.

[132] M. Zheng, G. Reader and G. Hawley, "Diesel engine exhaust gas recirculation-a review on advanced and novel concepts," Energy Conversion and Management, vol. 45, pp. 883-900, 2004.

[133] G. Abd-Alla, "Using exhaust gas recirculation in internal combustion engines: a review," Energy Conversion and Management, vol. 43, pp. 1027-1042, 2002.

[134] M. Fathi, R. Saray and M. Checkel, "The influence of Exhaust Gas Recirculation (EGR) on combustion and emissions of $\mathrm{n}$-heptane/natural gas fueled Homogeneous Charge Compression Ignition $(\mathrm{HCCl})$ engines," Appl Energy, vol. 88, no. 12,pp. 4719-4724, 2011.

[135] A. Deepak, S. Kumar and A. Kumar, "Effect of Exhaust Gas Recirculation (EGR) on performance, emission, deposits and durability of a constant speed compression ignition engine," Appl Energy, vol. 88, pp. 2900-2907, 2011.

[136] V. Khanna, "A Study of the Dynamics of Laminar and Turbulent Fully and Partially Premixed Flames," in Ph.D Dissertation, Virgina, Virgina Polytechnic Institute and State University, 2001.

[137] T. Lu and C. Law, "A criterion based on computational singular perturbation for the identification of quasi steady state species: A reduced mechanism for methane oxidation with NO chemistry," Combustion and Flame, vol. 154, no. 4, pp. 761-774, 2008.

[138] M. Marinov, "A Detailed Chemical Kinetic Model for High Temperature Ethanol Oxidation," Int. J. Chem. Kinet, vol. 31, no. UCRL-JC-131657, pp. 183-220, 1999. 
[139] H. Zhang, E. Hawkes, S. Kook, Z. Luo and T. Lu, " Computational investigations of the effects of thermal stratification in an ethanol-fuelled $\mathrm{HCCl}$ engine," Fuel, vol. submitted.

[140] H. Curran, J. Gaffuri, W. Pitz and C. Westbrook, "A Comprehensive Modeling Study of iso-Octane Oxidation," Combustion and Flame, vol. 129, pp. 253-280, 2002.

[141] H. Seiser, H. Pitsch, K. Seshadri, W. Pitz and H. Curran, "Extinction and Autoignition of n-Heptane in Counterflow Configuration," in Proceedings of the Combustion Institute 28 p. 2029-2037, Lawrence Livermore National Laboratory, Livermore, CA, 2000.

[142] M. Robinson, N. Clark and M. Alrbai, "Linear Power 2015," in Linear e-machine and free piston technology forum, University of Brighton, UK, 2015. 


\section{Chapter 10. Appendix}

\subsection{Functions and main MATLAB ${ }^{\circledR}$ code}

10.1.1 Stoichiometric combustion calculator

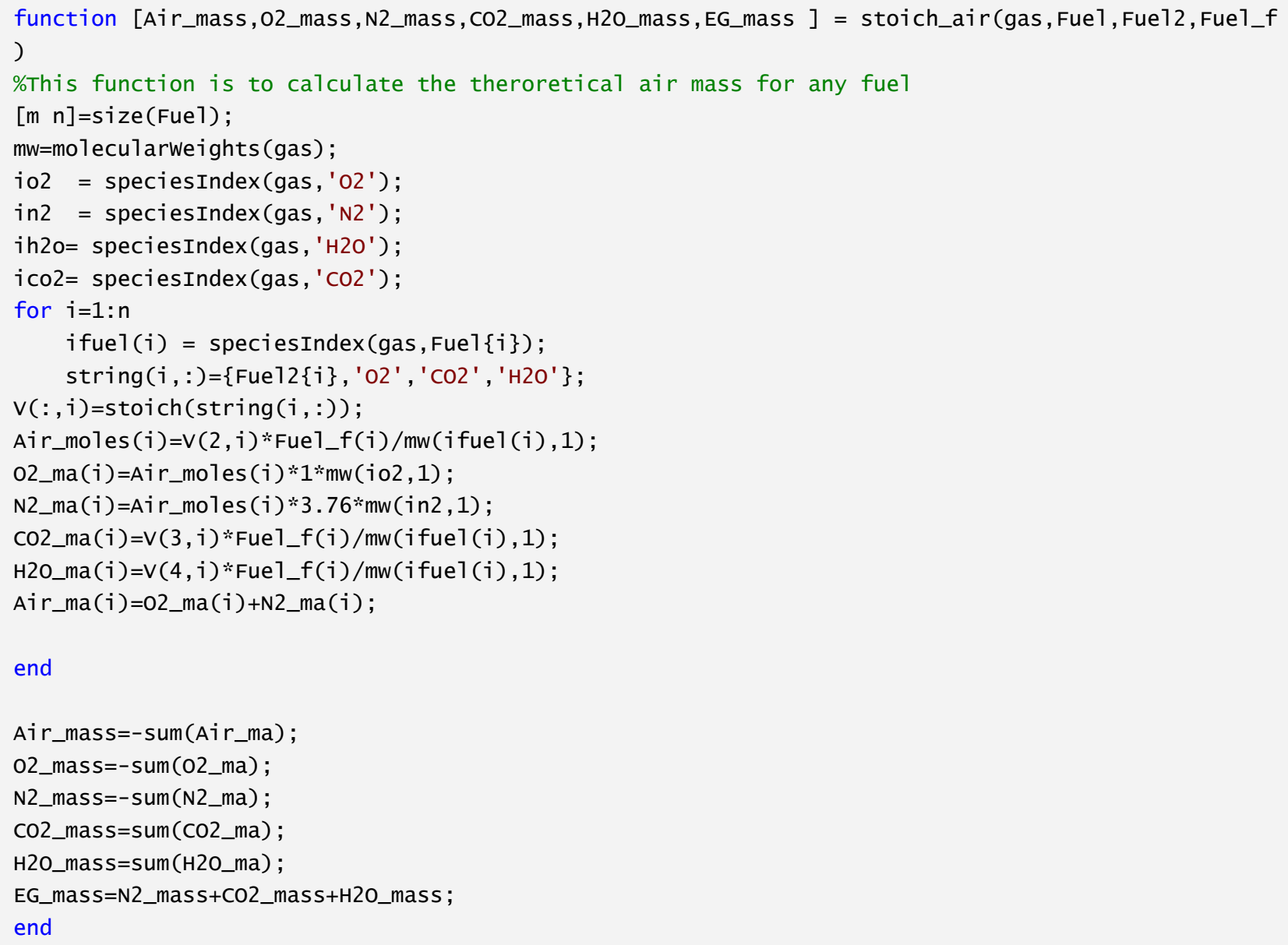


10.1.2 Piston position, velocity and acceleration calculation

function [V, endsim, x, v, a, Fs, Ff, Fw, Fp, Fg ] = Vol_Cr_MRC( P_new, vol, i , dt, x0, v0,a0, v_init )

global dir Smax bore dEP Area mass k kbias fco wa ca grav

if $i==1$

\section{Friction model parameters}

fmep $=150 * 1000 *$ Smax $; \%$ friction mean effective pressure

fco $=$ pi*bore^2/8*fmep;\%friction force coefficient $[\mathrm{N}]$

\%friction correlation as prescribed by Blair in Design and

\%simulation of Two-stroke Engines

Alternator model parameters

\% $A=25 ; \quad$ \%work force amplitude $[\mathrm{N}]$

Model

x_init =vol/Area;\%initial piston position [m]

$\mathrm{x}=$ x_init; \%position [m]

$v=v_{-}$init; $\quad$ \%velocity $[\mathrm{m} / \mathrm{s}]$

$V=p i *$ bore^ $2 / 4 * x$

$\operatorname{dir}=\operatorname{sign}(v)$;

$\mathrm{Fp}=\mathrm{pi} *$ bore ${ }^{\wedge} 2 / 4 * \mathrm{P} \_$new;

$[\mathrm{Ff}]=$ friction $(\mathrm{fco})$;

$[\mathrm{Fs}]=\operatorname{springs}(\mathrm{x}, \mathrm{k}, \mathrm{kbias})$;

$[\mathrm{Fw}]=\operatorname{work}(\mathrm{v}, \mathrm{Wa}, \mathrm{Ca})$;

$[\mathrm{Fg}]=-$ mass*grav;

$a=1 /$ mass $*(F s+F f+F w+F p+F g)$;

endsim $=0$;

$\%$ calculates forces and $a, v, x$

$v=v 0+a 0 * d t$; \%guesses for force calculations

$\mathrm{x}=\mathrm{x} 0+0.5 *(\mathrm{v}+\mathrm{v} 0) * \mathrm{dt}$;

P_mot $=$ P_new; $\%$ to be changed by the ode solver results

$\mathrm{Fp}=\mathrm{pi} *$ bore^2/4*P_mot;

$[\mathrm{Ff}]=\operatorname{friction}(\mathrm{fco})$;

$[\mathrm{Fs}]=\operatorname{springs}(\mathrm{x}, \mathrm{k}, \mathrm{kbias}) ;$

$[\mathrm{Fw}]=\operatorname{work}(\mathrm{v}, \mathrm{Wa}, \mathrm{Ca})$; 


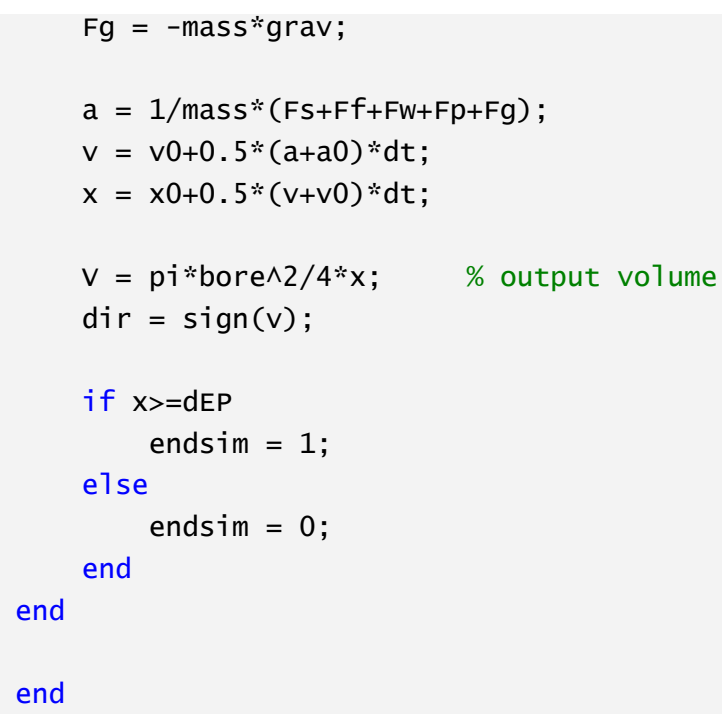


10.1.3 Main MATLAB ${ }^{\circledR}$ code for multi-cycle calculations

\section{Initialization}

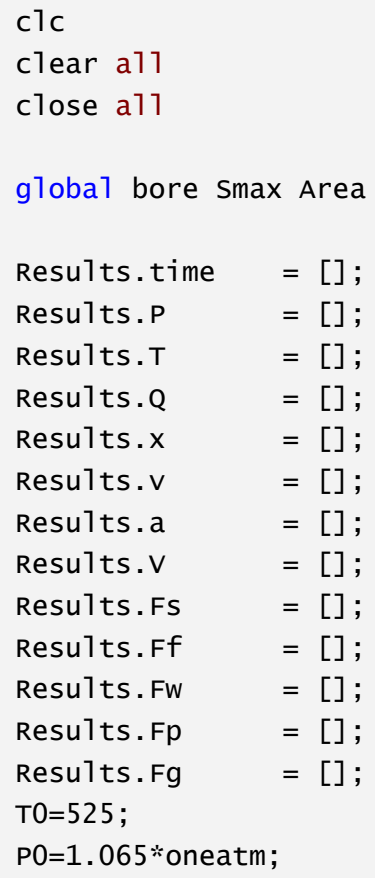

Engine parameters

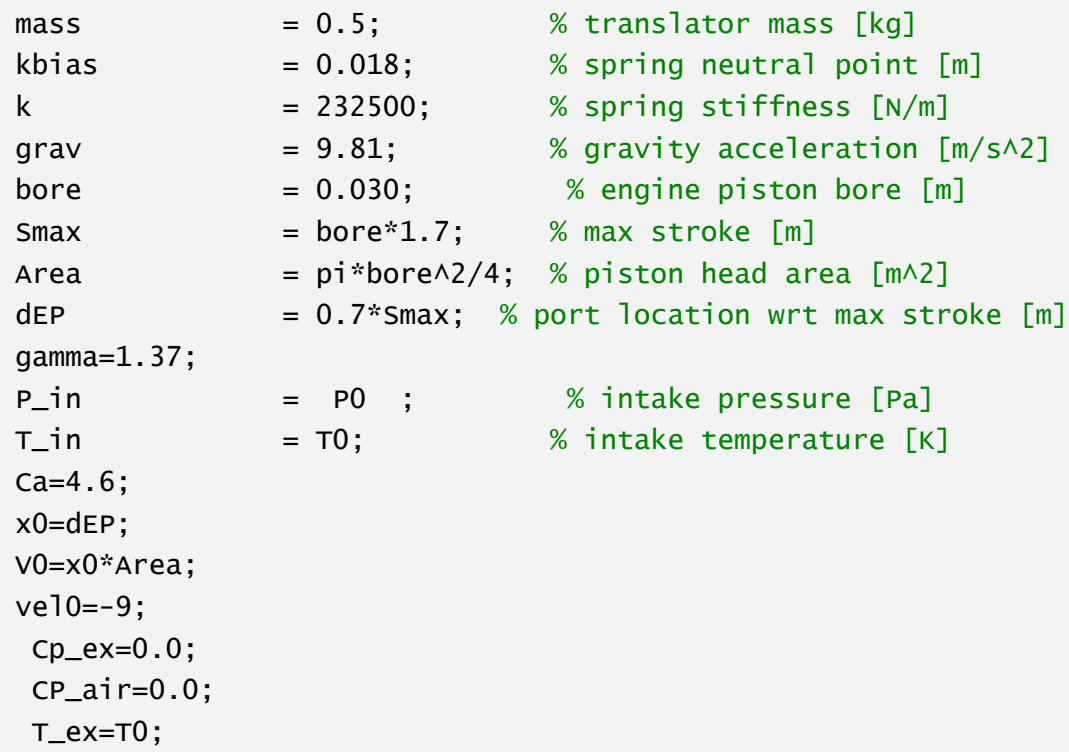




\section{Simulation}

Assumes the cycle begins with velocity $=0$ and $x 0=$ port closed position (dEP) to begin the compression stroke. Below, $\mathrm{xO}$ is parameterized according to the maximum allowable stroke (Smax).

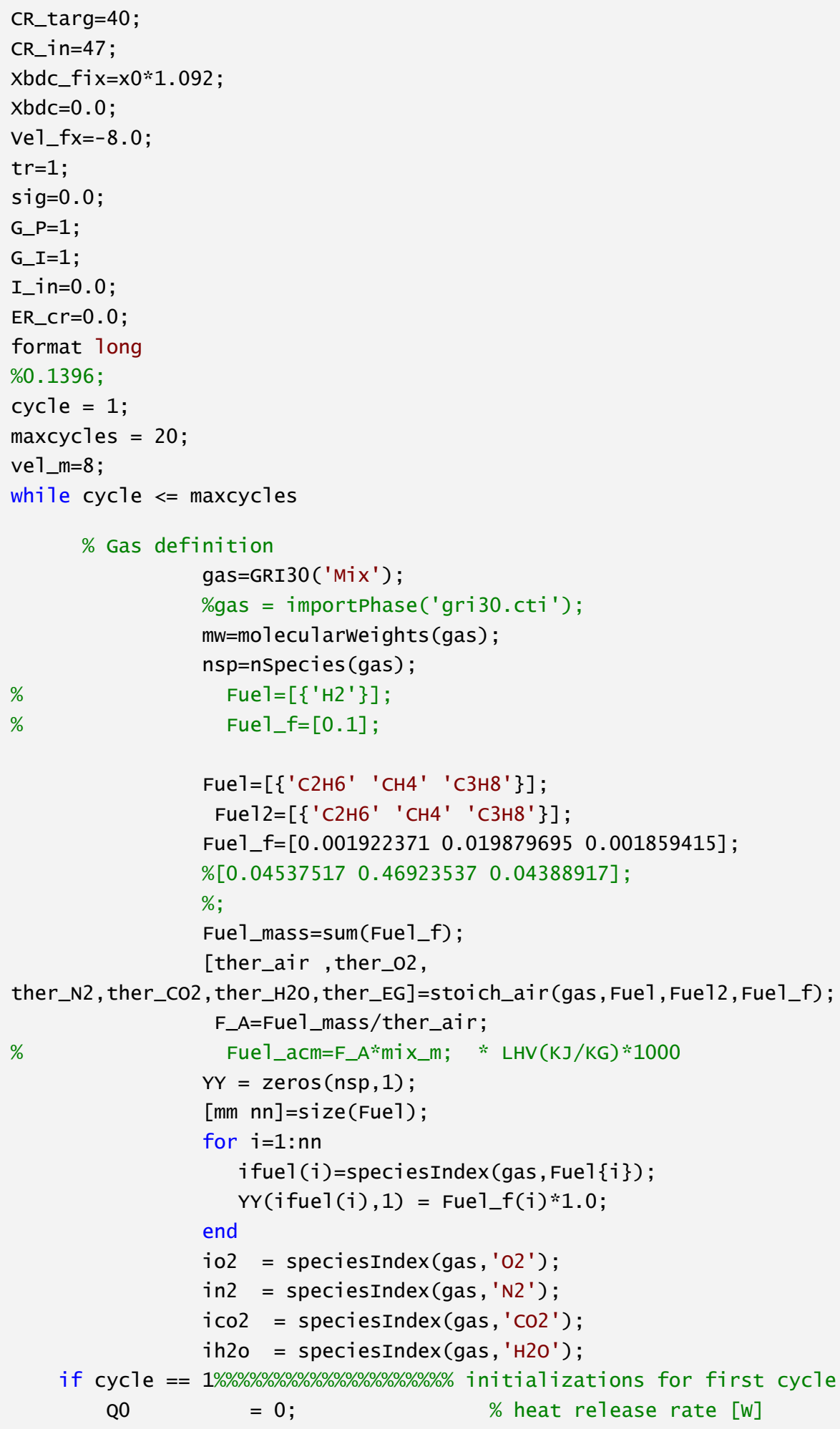




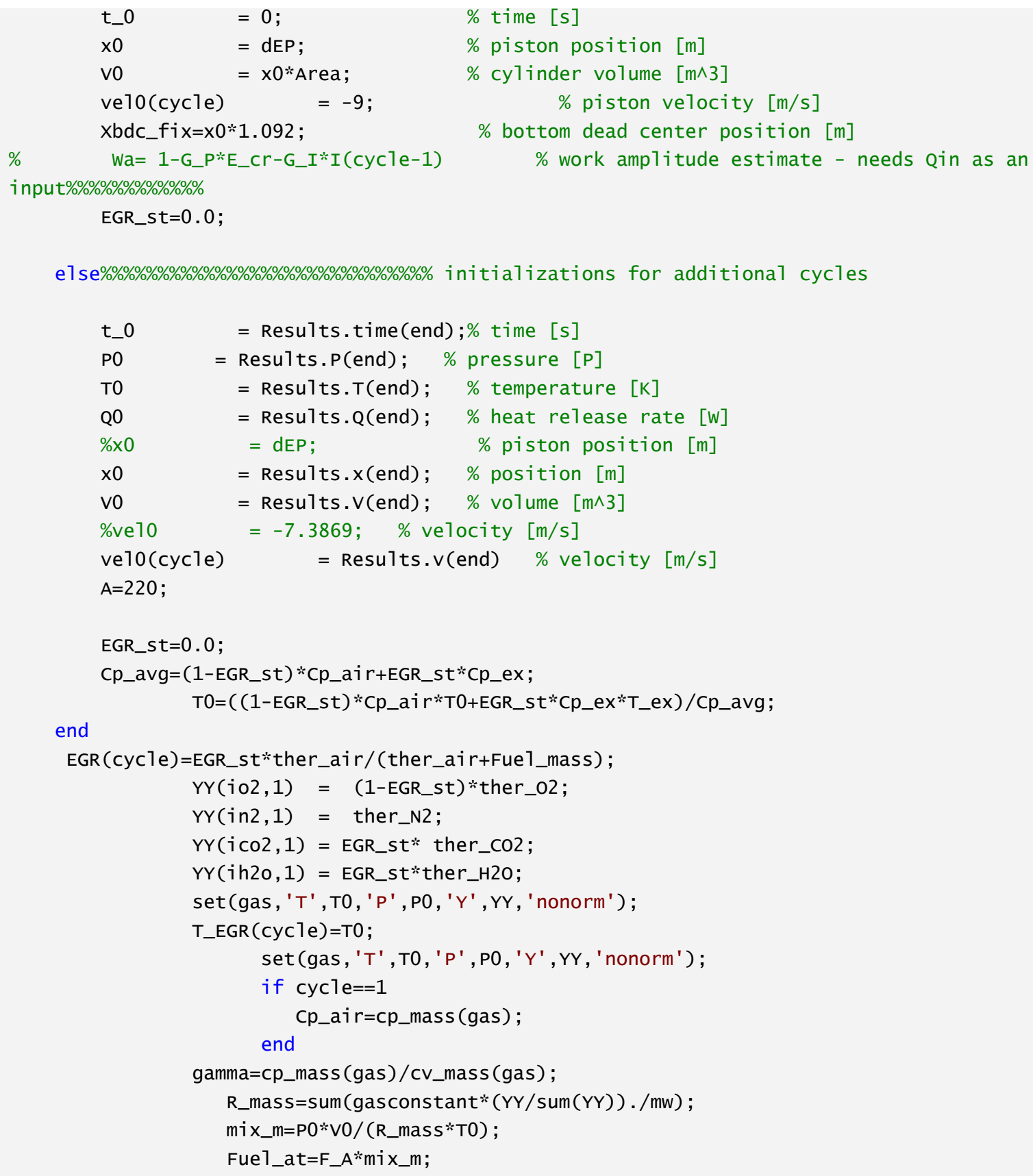

\section{Estimated volume profile}

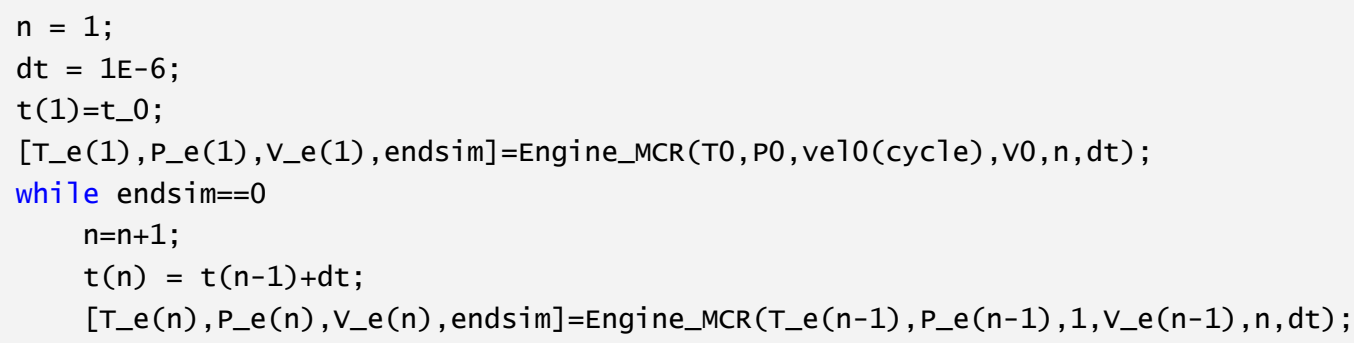




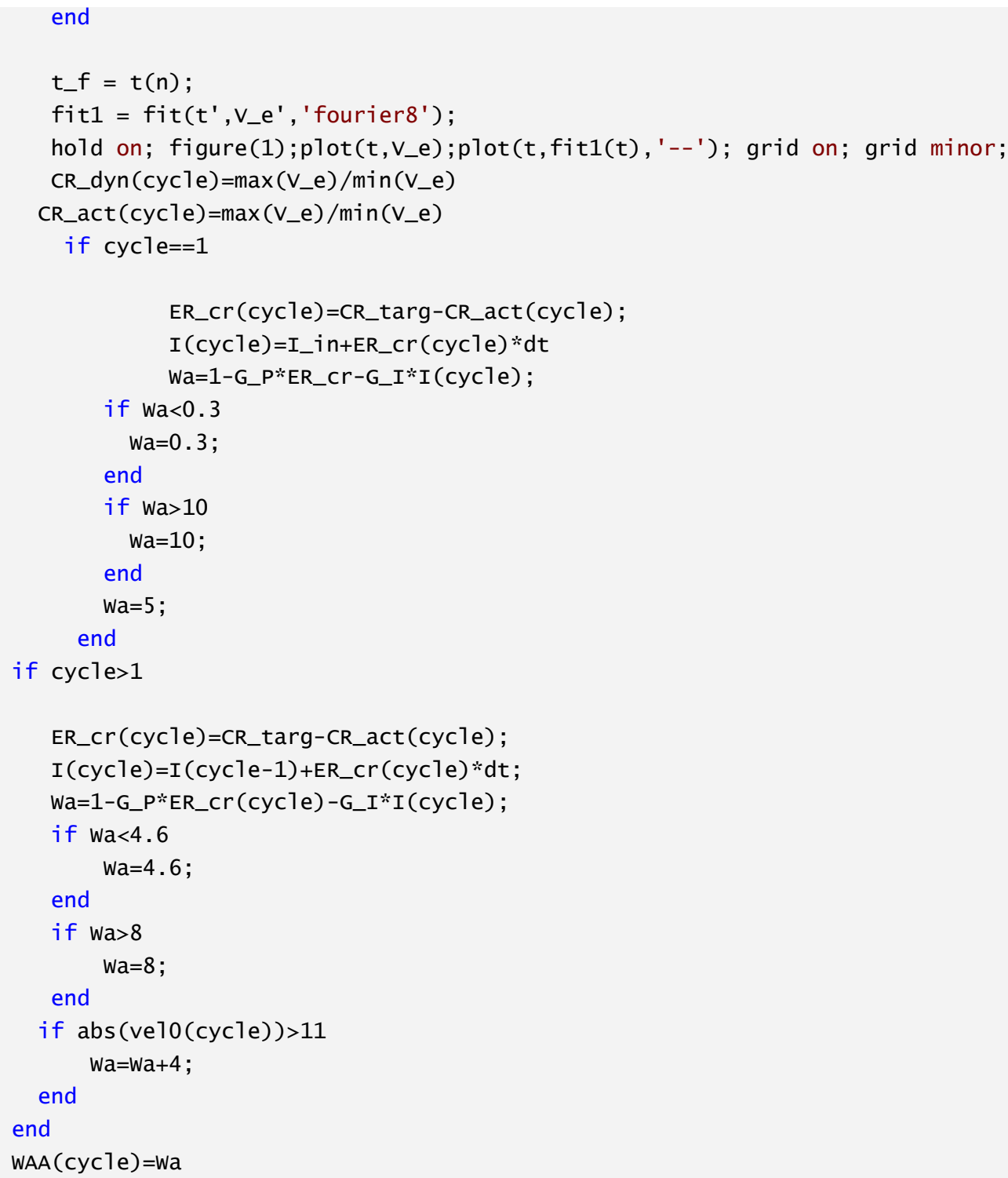

Actual volume profile with

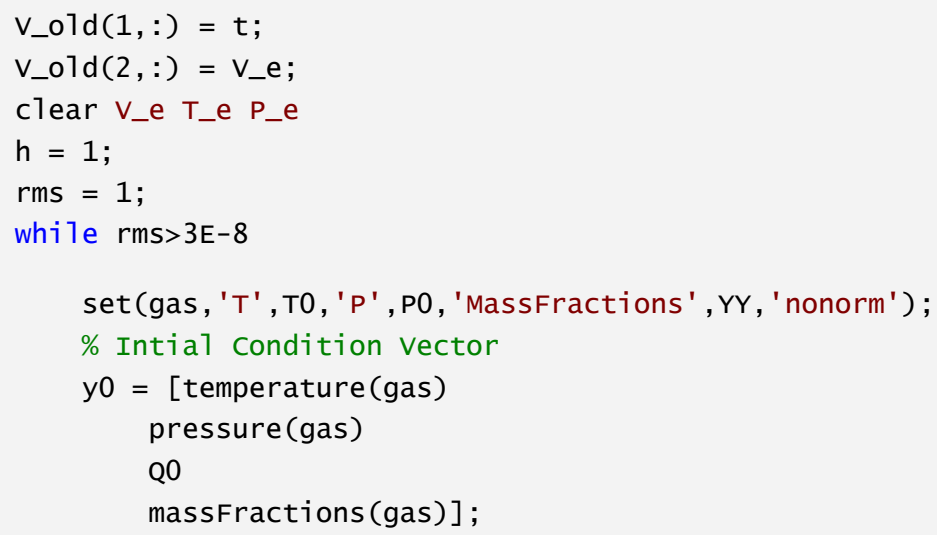




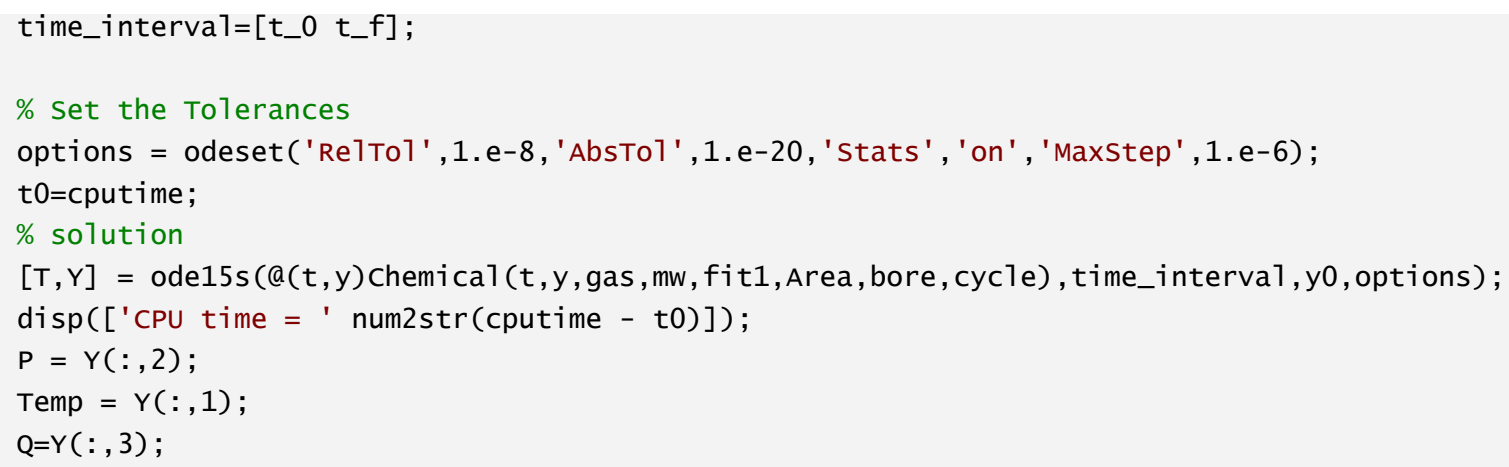

Volume profile correction

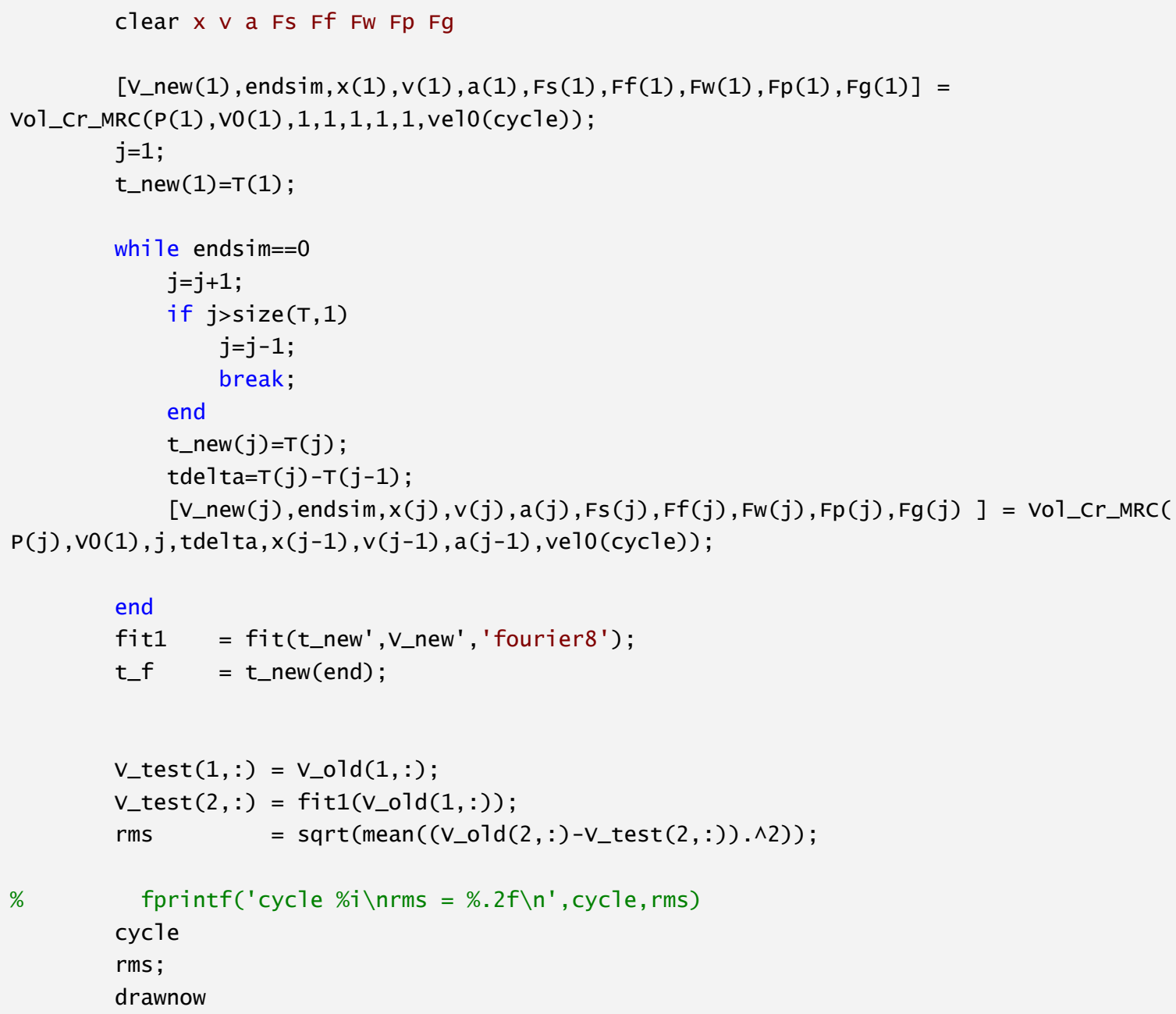

\section{Ending the cycle}

$\begin{array}{ll}\begin{array}{l}\text { \% Xbdc_old } \\ \text { endsim }\end{array} & =0 ; \\ \text { t_neww } & =T(1: j)^{\prime} ;\end{array}$




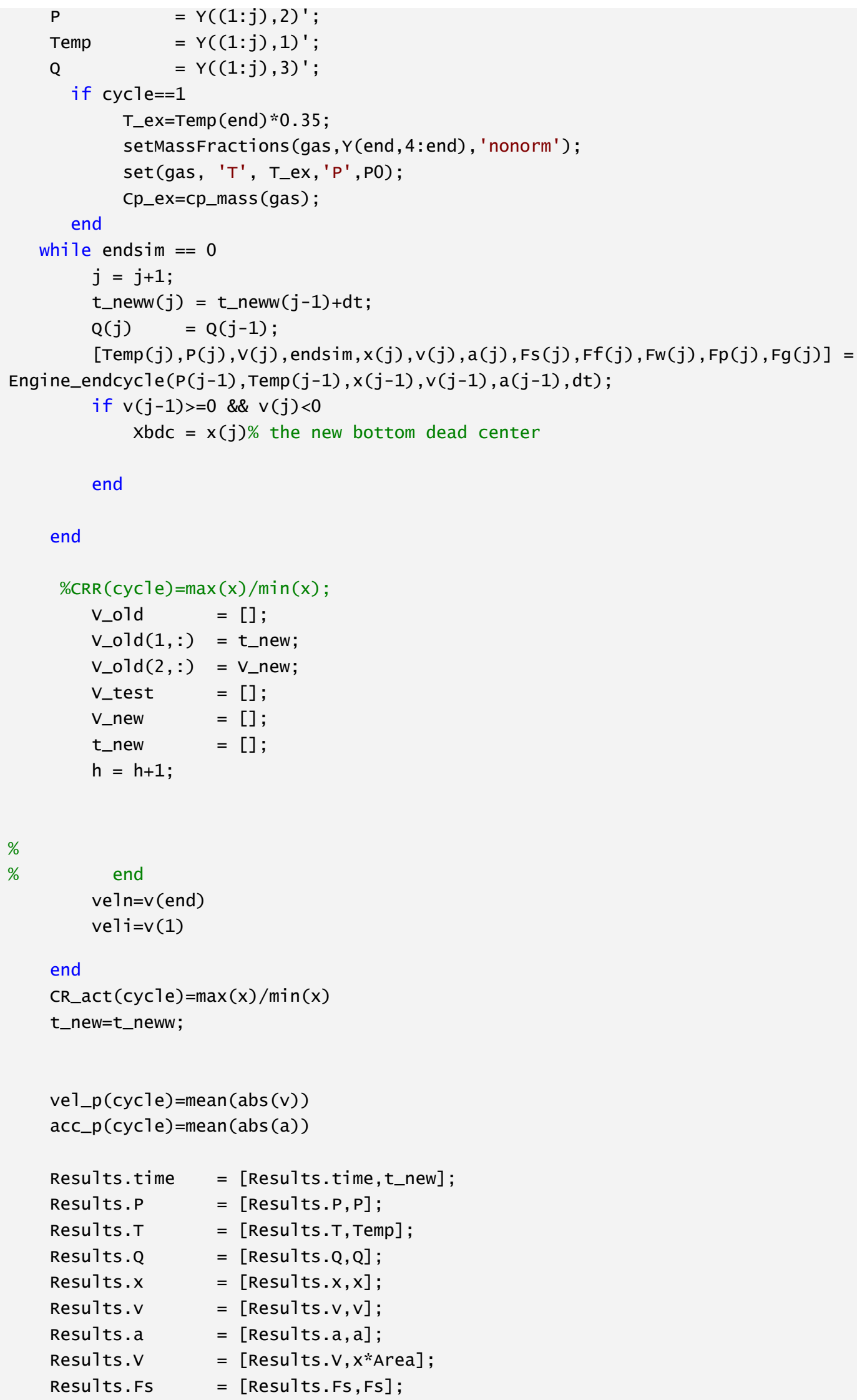




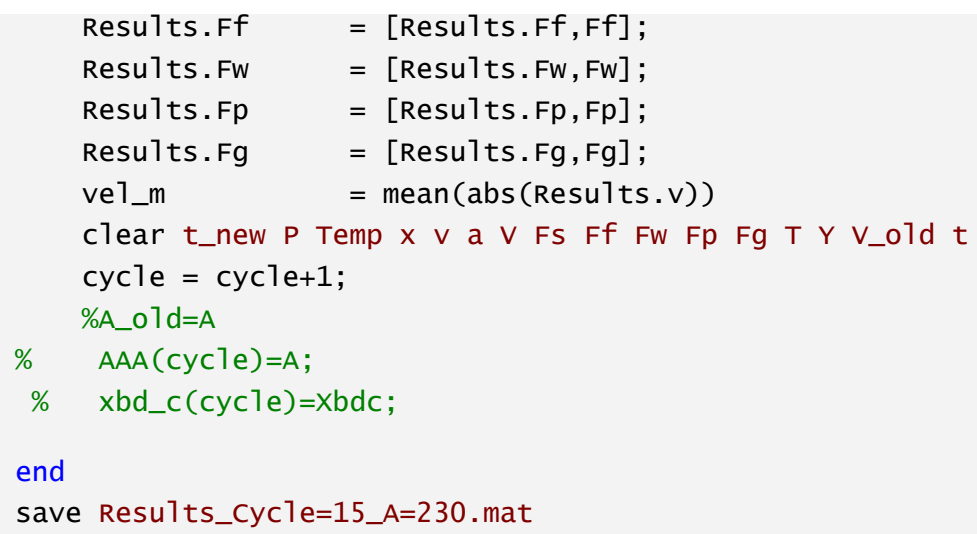

\section{Energy analysis}

$\%$ LHV = fue 1 LHV in $\mathrm{kJ} / \mathrm{kg}$

Energy.Fue $1=1000 *$ Fue 1 _at* $($ cycle -1$) * 47000 ; \% 47000$ is LHV

Energy. HR = Results.Q(end);

Energy.A1t $=$ abs (trapz(Resu1ts.x, Resu1ts.Fw));

Energy. Fric $=\operatorname{abs}(\operatorname{trapz}($ Results.x, Results.Ff $))$;

\section{Plotting}

close a11

figure; plot (Results. $x$, Results.v)

xlabel ('position [m] ')

ylabel ('velocity $[\mathrm{m} / \mathrm{s}]$ ')

grid on

grid minor

figure; plot (Results.v, Results.a)

xlabel ('velocity $[\mathrm{m} / \mathrm{s}]$ ')

ylabel ('acceleration [m/s^2]')

grid on

grid minor

figure (3); hold on

plot (Results.time, Resu1ts.Q)

xlabel ('time [s]')

ylabel ('total heat release [J]')

grid on

grid minor

hold off

figure;

plot ((Results.x), (Results.P))

set (gca, 'yscale', ' log')

xlabel ('position [m] ')

ylabel ('pressure $[\mathrm{Pa}]^{\prime}$ )

grid on 
grid minor

figure

s1=subplot $(3,1,1)$;

plot (Results.time, Resu1ts. $x$ )

ylabel ('position [m]')

grid on

grid minor

s2=subplot $(3,1,2)$;

plot (Results.time, Resu1ts.v)

ylabel ('velocity $[\mathrm{m} / \mathrm{s}]$ ')

grid on

grid minor

s3=subplot $(3,1,3)$;

plot (Results.time, Results.a)

xlabel ('time [s]')

ylabel ('accleration $[\mathrm{m} / \mathrm{s} \wedge 2]$ ')

grid on

grid minor

1inkaxes([s1 s2 s3], 'x')

\section{figure;}

s1=subplot $(2,1,1)$;

plot (Results.time, Results.P)

set (gca, 'yscale', ' log')

ylabel ('pressure [Pa]')

grid on

grid minor

s2=subplot $(2,1,2)$;

plot (Results.time, Results.T)

xlabel ('time [s]')

ylabel ('temperature $[\mathrm{K}]$ ')

grid on

grid minor

1inkaxes ([s1 s2], 'x')

print ('example', '-dpng', '-r1200')

figure;

plot (Results.time, Results. Fw, ...

Resu1ts.time, Resu7ts.Fs, ...

Results.time, Results.Ff, ...

Results.time, Resu1ts.Fp) 
10.2 Modified version of GRI 3.0 mechanism (species reactions)

ELEMENTS

O $\quad$ H $\quad C \quad N \quad A R$

END

SPECIES

$\begin{array}{llllllll}\mathrm{H} 2 & \mathrm{H} & \mathrm{O} & \mathrm{O} 2 & \mathrm{OH} & \mathrm{H} 2 \mathrm{O} & \mathrm{HO} 2 & \mathrm{H} 2 \mathrm{O} 2 \\ \mathrm{C} & \mathrm{CH} & \mathrm{CH} 2 & \mathrm{CH} 2(\mathrm{~S}) & \mathrm{CH} 3 & \mathrm{CH} 4 & \mathrm{CO} & \mathrm{CO} 2 \\ \mathrm{HCO} & \mathrm{CH} 2 \mathrm{O} & \mathrm{CH} 2 \mathrm{OH} & \mathrm{CH} 3 \mathrm{O} & \mathrm{CH} 3 \mathrm{OH} & \mathrm{C} 2 \mathrm{H} & \mathrm{C} 2 \mathrm{H} 2 & \mathrm{C} 2 \mathrm{H} 3 \\ \mathrm{C} 2 \mathrm{H} 4 & \mathrm{C} 2 \mathrm{H} 5 & \mathrm{C} 2 \mathrm{H} 6 & \mathrm{HCCO} & \mathrm{CH} 2 \mathrm{CO} & \mathrm{HCCOH} & \mathrm{N} & \mathrm{NH} \\ \mathrm{NH} 2 & \mathrm{NH} 3 & \mathrm{NNH} & \mathrm{NO} & \mathrm{NO} 2 & \mathrm{~N} 2 \mathrm{O} & \mathrm{HNO} & \mathrm{CN} \\ \mathrm{HCN} & \mathrm{H} 2 \mathrm{CN} & \mathrm{HCNN} & \mathrm{HCNO} & \mathrm{HOCN} & \mathrm{HNCO} & \mathrm{NCO} & \mathrm{N} 2 \\ \mathrm{AR} & \mathrm{C} 3 \mathrm{H} 7 & \mathrm{C} 3 \mathrm{H} 8 & \mathrm{CH} 2 \mathrm{CHO} & \mathrm{CH} 3 \mathrm{CHO} & & & \end{array}$

END

! THERMO

! Insert GRI-Mech thermodynamics here or use in default file

! END

REACTIONS

$2 \mathrm{O}+\mathrm{M}<=>\mathrm{O} 2+\mathrm{M}$

$1.200 \mathrm{E}+17-1.000$

.00

$\mathrm{H} 2 / 2.40 / \mathrm{H} 2 \mathrm{O} / 15.40 / \mathrm{CH} 4 / 2.00 / \mathrm{CO} / 1.75 / \mathrm{CO} 2 / 3.60 / \mathrm{C} 2 \mathrm{H} 6 /$ $3.00 / \mathrm{AR} / .83 /$

$\mathrm{O}+\mathrm{H}+\mathrm{M}<=>\mathrm{OH}+\mathrm{M} \quad 5.000 \mathrm{E}+17 \quad-1.000$

.00

$\mathrm{H} 2 / 2.00 / \mathrm{H} 2 \mathrm{O} / 6.00 / \mathrm{CH} 4 / 2.00 / \mathrm{CO} / 1.50 / \mathrm{CO} 2 / 2.00 / \mathrm{C} 2 \mathrm{H} 6 / 3.00 / \mathrm{AR} /$ $.70 / \mathrm{O}+\mathrm{H} 2<=>\mathrm{H}+\mathrm{OH}$ $3.870 \mathrm{E}+04$

$2.700 \quad 6260.00$

$\mathrm{O}+\mathrm{HO} 2<=>\mathrm{OH}+\mathrm{O} 2$

$2.000 \mathrm{E}+13 \quad .000$

.00

$\mathrm{O}+\mathrm{H} 2 \mathrm{O} 2<=>\mathrm{OH}+\mathrm{HO} 2$

4000.00

$9.630 E+06 \quad 2.000$ 


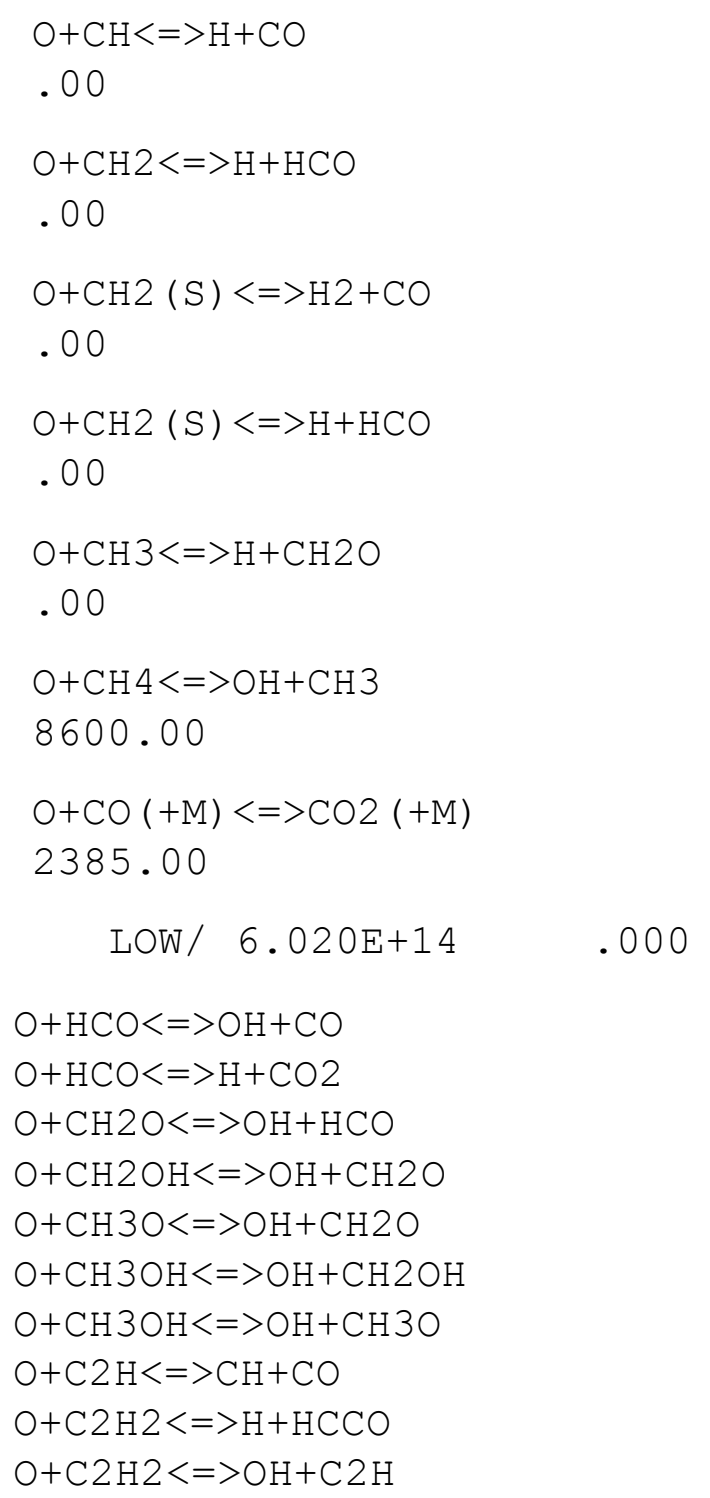

$5.700 E+13 \quad .000$

$8.000 \mathrm{E}+13 \quad .000$

$1.500 \mathrm{E}+13 \quad .000$

$1.500 \mathrm{E}+13 \quad .000$

$5.060 \mathrm{E}+13 \quad .000$

1.020E+09 1.500

$1.800 \mathrm{E}+10 \quad .000$

$\begin{array}{lll}3.000 \mathrm{E}+13 & .000 & .00 \\ 3.000 \mathrm{E}+13 & .000 & .00 \\ 3.900 \mathrm{E}+13 & .000 & 3540.00 \\ 1.000 \mathrm{E}+13 & .000 & .00 \\ 1.000 \mathrm{E}+13 & .000 & .00 \\ 3.880 \mathrm{E}+05 & 2.500 & 3100.00 \\ 1.300 \mathrm{E}+05 & 2.500 & 5000.00 \\ 5.000 \mathrm{E}+13 & .000 & .00 \\ 1.350 \mathrm{E}+07 & 2.000 & 1900.00 \\ 4.600 \mathrm{E}+19 & -1.410 & 28950.00\end{array}$

$\mathrm{CH} 4 / 2.00 / \mathrm{CO} / 1.50 / \mathrm{CO} 2 / 3.50 /$ 


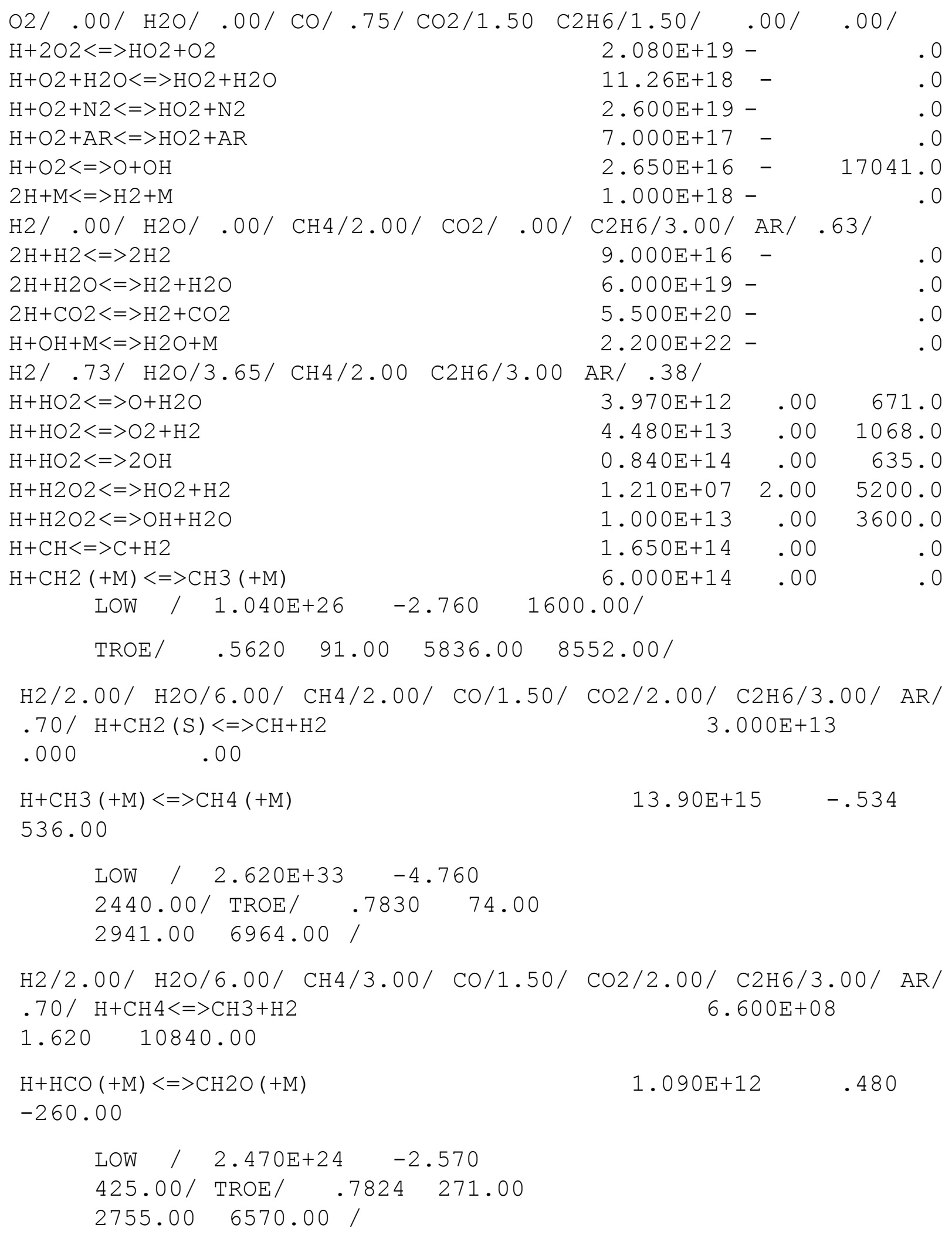


$\mathrm{H} 2 / 2.00 / \mathrm{H} 2 \mathrm{O} / 6.00 / \mathrm{CH} 4 / 2.00 / \mathrm{CO} / 1.50 / \mathrm{CO} 2 / 2.00 / \mathrm{C} 2 \mathrm{H} 6 / 3.00 / \mathrm{AR} /$ $.70 / \mathrm{H}+\mathrm{HCO}<=>\mathrm{H} 2+\mathrm{CO}$ $7.340 \mathrm{E}+13$

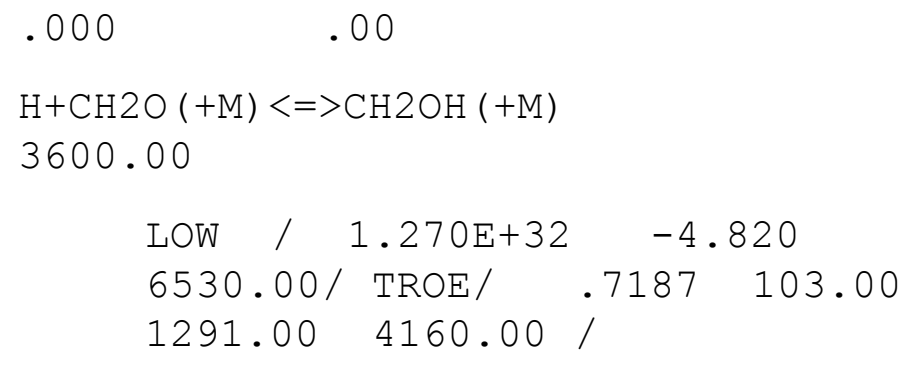




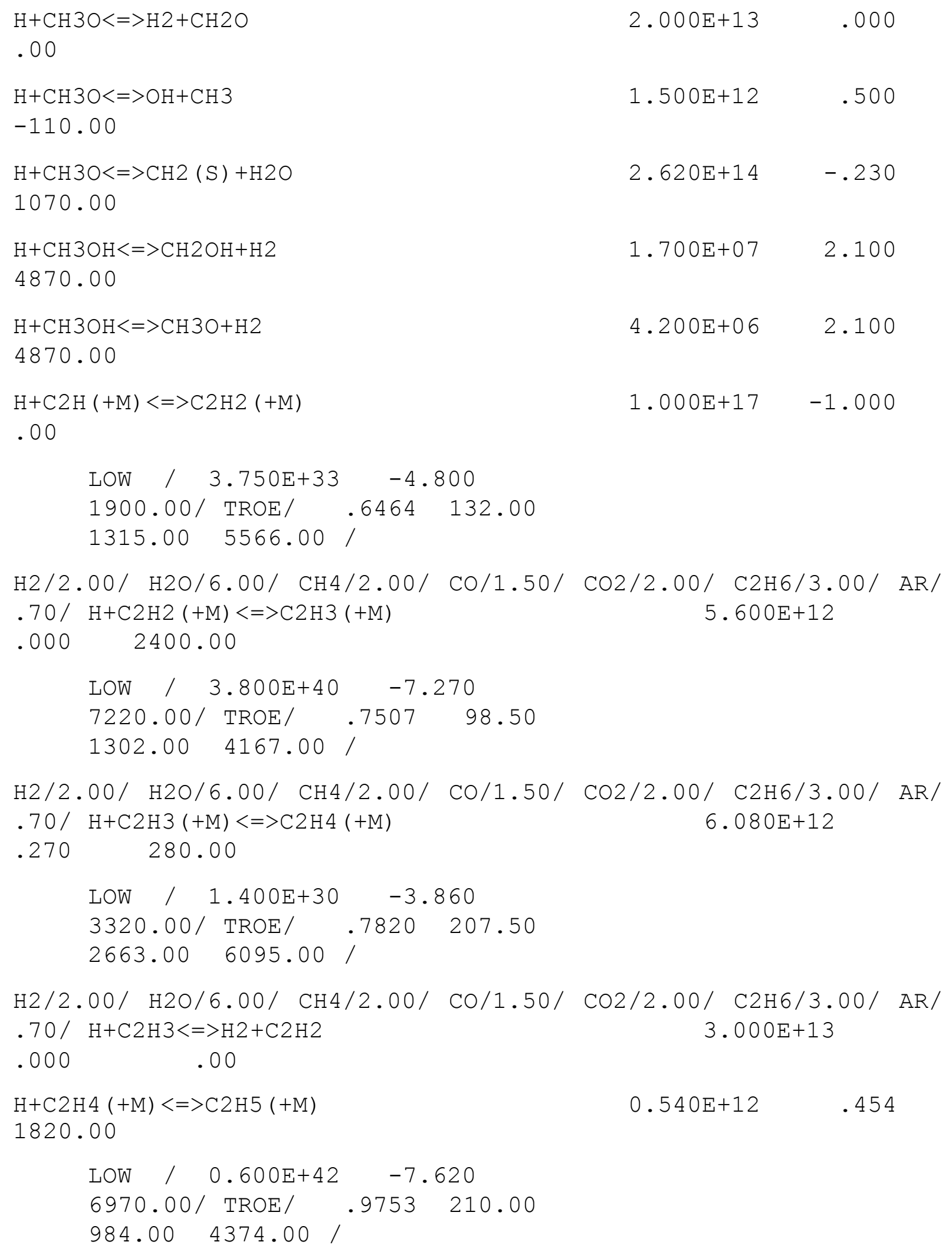


$\mathrm{H} 2 / 2.00 / \mathrm{H} 2 \mathrm{O} / 6.00 / \mathrm{CH} 4 / 2.00 / \mathrm{CO} / 1.50 / \mathrm{CO} 2 / 2.00 / \mathrm{C} 2 \mathrm{H} 6 / 3.00 / \mathrm{AR} /$ $.70 / \mathrm{H}+\mathrm{C} 2 \mathrm{H} 4<=>\mathrm{C} 2 \mathrm{H} 3+\mathrm{H} 2$ $1.325 \mathrm{E}+06$ $2.530 \quad 12240.00$

$\mathrm{H}+\mathrm{C} 2 \mathrm{H} 5(+\mathrm{M})<=>\mathrm{C} 2 \mathrm{H} 6(+\mathrm{M})$ 1580.00

$$
5.210 \mathrm{E}+17-.990
$$

$\begin{array}{llll}\text { LOW / } & 1.990 \mathrm{E}+41 & -7.080 \\ 6685.00 / \mathrm{TROE} / & .8422 & 125.00 \\ 2219.00 & 6882.00 / & \end{array}$

\begin{tabular}{|c|c|}
\hline $\mathrm{H}+\mathrm{C} 2 \mathrm{H} 5<=>\mathrm{H} 2+\mathrm{C} 2 \mathrm{H} 4$ & $2.000 \mathrm{E}$ \\
\hline $\mathrm{H}+\mathrm{C} 2 \mathrm{H} 6<=>\mathrm{C} 2 \mathrm{H} 5+\mathrm{H} 2$ & $1.150 \mathrm{E}$ \\
\hline $\mathrm{H}+\mathrm{HCCO}<=>\mathrm{CH} 2(\mathrm{~S})+\mathrm{CO}$ & 1.000 \\
\hline $\mathrm{H}+\mathrm{CH} 2 \mathrm{CO}<=>\mathrm{HCCO}+\mathrm{H} 2$ & .0 \\
\hline $\mathrm{H}+\mathrm{CH} 2 \mathrm{CO}<=>\mathrm{CH} 3+\mathrm{CO}$ & 1 \\
\hline $\mathrm{H}+\mathrm{HCCOH}<=>\mathrm{H}+\mathrm{CH} 2 \mathrm{CO}$ & \\
\hline $\mathrm{H} 2+\mathrm{CO}(+\mathrm{M})<=>\mathrm{CH} 2 \mathrm{O}(+\mathrm{M})$ & \\
\hline $\begin{array}{l}\mathrm{H} 2 / 2.00 / \mathrm{H} 2 \mathrm{O} / 6.00 / \mathrm{CH} 4 / 2.00 / \\
.70 /\end{array}$ & 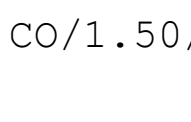 \\
\hline $\begin{array}{l}\text { LOW / } 5.070 E+27,-3.4 \\
84350.00 / \text { TROE/ } .9320 \\
1540.0010300 .00 /\end{array}$ & \\
\hline
\end{tabular}

$\mathrm{H} 2 / 2.00 / \mathrm{H} 2 \mathrm{O} / 6.00 / \mathrm{CH} 4 / 2.00 / \mathrm{CO} / 1.50 / \mathrm{CO} 2 / 2.00 / \mathrm{C} 2 \mathrm{H} 6 / 3.00 / \mathrm{AR} /$ $.70 / \mathrm{OH}+\mathrm{H} 2<=>\mathrm{H}+\mathrm{H} 2 \mathrm{O}$ $2.160 \mathrm{E}+08$ $1.510 \quad 3430.00$ $2 \mathrm{OH}(+\mathrm{M})<=>\mathrm{H} 2 \mathrm{O} 2(+\mathrm{M}) \quad 7.400 \mathrm{E}+13 \quad-.370$ .00

$$
\begin{aligned}
& \text { LOW / } 2.300 \mathrm{E}+18 \quad-.900- \\
& 1700.00 / \text { TROE/ } / .734694 .00 \\
& 1756.00 \text { 5182.00/ }
\end{aligned}
$$

$\mathrm{H} 2 / 2.00 / \mathrm{H} 2 \mathrm{O} / 6.00 / \mathrm{CH} 4 / 2.00 / \mathrm{CO} / 1.50 / \mathrm{CO} 2 / 2.00 / \mathrm{C} 2 \mathrm{H} 6 / 3.00 / \mathrm{AR} /$ $.70 /$
$2 \mathrm{OH}<=>\mathrm{O}+\mathrm{H} 2 \mathrm{O}$
$3.570 \mathrm{E}+04 \quad 2.400$
$-2110.00$
$\mathrm{OH}+\mathrm{HO} 2<=>\mathrm{O} 2+\mathrm{H} 2 \mathrm{O}$
$1.450 \mathrm{E}+13 \quad .000$
$-500.00$
DUPLICATE
$\mathrm{OH}+\mathrm{H} 2 \mathrm{O} 2<=>\mathrm{HO} 2+\mathrm{H} 2 \mathrm{O}$
$2.000 \mathrm{E}+12 \quad .000$
427.00 


\begin{tabular}{|c|c|c|}
\hline $\begin{array}{l}\mathrm{OH}+\mathrm{H} 2 \mathrm{O} 2<=>\mathrm{HO} 2+\mathrm{H} 2 \mathrm{O} \\
29410.00\end{array}$ & $1.700 \mathrm{E}+18$ & .000 \\
\hline DUPLICATE & & \\
\hline $\begin{array}{l}\mathrm{OH}+\mathrm{C}<=>\mathrm{H}+\mathrm{CO} \\
.00\end{array}$ & $5.000 \mathrm{E}+13$ & .000 \\
\hline $\begin{array}{l}\mathrm{OH}+\mathrm{CH}<=>\mathrm{H}+\mathrm{HCO} \\
.00\end{array}$ & $3.000 E+13$ & .000 \\
\hline $\begin{array}{l}\mathrm{OH}+\mathrm{CH} 2<=>\mathrm{H}+\mathrm{CH} 2 \mathrm{O} \\
.00\end{array}$ & $2.000 E+13$ & .000 \\
\hline $\begin{array}{l}\mathrm{OH}+\mathrm{CH} 2<=>\mathrm{CH}+\mathrm{H} 2 \mathrm{O} \\
3000.00\end{array}$ & $1.130 \mathrm{E}+07$ & 2.000 \\
\hline $\begin{array}{l}\mathrm{OH}+\mathrm{CH} 2(\mathrm{~S})<=>\mathrm{H}+\mathrm{CH} 2 \mathrm{O} \\
.00\end{array}$ & $3.000 \mathrm{E}+13$ & .000 \\
\hline $\begin{array}{l}\mathrm{OH}+\mathrm{CH} 3(+\mathrm{M})<=>\mathrm{CH} 3 \mathrm{OH}(+\mathrm{M}) \\
1330.00\end{array}$ & $2.790 \mathrm{E}+18$ & -1.430 \\
\hline $\begin{array}{l}\text { LOW / } / .000 \mathrm{E}+36 \quad-5.920 \\
3140.00 / \text { TROE/ .4120 } 195.0 \\
5900.00 \quad 6394.00 /\end{array}$ & & \\
\hline $\begin{array}{l}\mathrm{H} 2 / 2.00 / \mathrm{H} 2 \mathrm{O} / 6.00 / \mathrm{CH} 4 / 2.00 / \mathrm{CO} / 1.50 / \\
\mathrm{OH}+\mathrm{CH} 3<=>\mathrm{CH} 2+\mathrm{H} 2 \mathrm{O} \\
5420.00\end{array}$ & $\begin{array}{r}\mathrm{CO} 2 / 2.00 / \mathrm{C} 2 \\
5.600 \mathrm{E}+07\end{array}$ & $\begin{array}{l}3.00 / \\
1.600\end{array}$ \\
\hline $\begin{array}{l}\mathrm{OH}+\mathrm{CH} 3<=>\mathrm{CH} 2(\mathrm{~S})+\mathrm{H} 2 \mathrm{O} \\
1417.00\end{array}$ & $6.440 \mathrm{E}+17$ & -1.340 \\
\hline $\begin{array}{l}\mathrm{OH}+\mathrm{CH} 4<=>\mathrm{CH} 3+\mathrm{H} 2 \mathrm{O} \\
3120.00\end{array}$ & $1.000 \mathrm{E}+08$ & 1.600 \\
\hline $\begin{array}{l}\mathrm{OH}+\mathrm{CO}<=>\mathrm{H}+\mathrm{CO} 2 \\
70.00\end{array}$ & $4.760 E+07$ & 1.228 \\
\hline $\begin{array}{l}\mathrm{OH}+\mathrm{HCO}<=>\mathrm{H} 2 \mathrm{O}+\mathrm{CO} \\
.00\end{array}$ & $5.000 \mathrm{E}+13$ & .000 \\
\hline $\begin{array}{l}\mathrm{OH}+\mathrm{CH} 2 \mathrm{O}<=>\mathrm{HCO}+\mathrm{H} 2 \mathrm{O} \\
-447.00\end{array}$ & $3.430 E+09$ & 1.180 \\
\hline $\begin{array}{l}\mathrm{OH}+\mathrm{CH} 2 \mathrm{OH}<=>\mathrm{H} 2 \mathrm{O}+\mathrm{CH} 2 \mathrm{O} \\
.00\end{array}$ & $5.000 \mathrm{E}+12$ & .000 \\
\hline
\end{tabular}




\begin{tabular}{|c|c|c|c|c|}
\hline $\begin{array}{l}\mathrm{OH}+\mathrm{CH} 3 \mathrm{O}<=>\mathrm{H} 2 \mathrm{O}+\mathrm{CH} 2 \mathrm{O} \\
.00\end{array}$ & & \multicolumn{2}{|c|}{$5.000 \mathrm{E}+12$} & .000 \\
\hline $\begin{array}{l}\mathrm{OH}+\mathrm{CH} 3 \mathrm{OH}<=>\mathrm{CH} 2 \mathrm{OH}+\mathrm{H} 2 \mathrm{O} \\
-840.00\end{array}$ & & \multicolumn{2}{|c|}{$1.440 \mathrm{E}+06$} & 2.000 \\
\hline $\begin{array}{l}\mathrm{OH}+\mathrm{CH} 3 \mathrm{OH}<=>\mathrm{CH} 3 \mathrm{O}+\mathrm{H} 2 \mathrm{O} \\
1500.00\end{array}$ & & \multicolumn{2}{|c|}{$6.300 \mathrm{E}+06$} & 2.000 \\
\hline $\begin{array}{l}\mathrm{OH}+\mathrm{C} 2 \mathrm{H}<=>\mathrm{H}+\mathrm{HCCO} \\
.00\end{array}$ & & \multicolumn{2}{|c|}{$2.000 \mathrm{E}+13$} & .000 \\
\hline $\begin{array}{l}\mathrm{OH}+\mathrm{C} 2 \mathrm{H} 2<=>\mathrm{H}+\mathrm{CH} 2 \mathrm{CO} \\
-1000.00\end{array}$ & & \multicolumn{2}{|c|}{$2.180 \mathrm{E}-04$} & 4.500 \\
\hline $\begin{array}{l}\mathrm{OH}+\mathrm{C} 2 \mathrm{H} 2<=>\mathrm{H}+\mathrm{HCCOH} \\
13500.00\end{array}$ & & \multicolumn{2}{|c|}{$5.040 \mathrm{E}+05$} & 2.300 \\
\hline $\begin{array}{l}\mathrm{OH}+\mathrm{C} 2 \mathrm{H} 2<=>\mathrm{C} 2 \mathrm{H}+\mathrm{H} 2 \mathrm{O} \\
14000.00\end{array}$ & & \multicolumn{2}{|c|}{$3.370 \mathrm{E}+07$} & 2.000 \\
\hline $\begin{array}{l}\mathrm{OH}+\mathrm{C} 2 \mathrm{H} 2<=>\mathrm{CH} 3+\mathrm{CO} \\
-2000.00\end{array}$ & & \multicolumn{2}{|c|}{$4.830 \mathrm{E}-04$} & 4.000 \\
\hline $\begin{array}{l}\mathrm{OH}+\mathrm{C} 2 \mathrm{H} 3<=>\mathrm{H} 2 \mathrm{O}+\mathrm{C} 2 \mathrm{H} 2 \\
.00\end{array}$ & & \multicolumn{2}{|c|}{$5.000 \mathrm{E}+12$} & .000 \\
\hline $\begin{array}{l}\mathrm{OH}+\mathrm{C} 2 \mathrm{H} 4<=>\mathrm{C} 2 \mathrm{H} 3+\mathrm{H} 2 \mathrm{O} \\
2500.00\end{array}$ & & \multicolumn{2}{|c|}{$3.600 \mathrm{E}+06$} & 2.000 \\
\hline $\begin{array}{l}\mathrm{OH}+\mathrm{C} 2 \mathrm{H} 6<=>\mathrm{C} 2 \mathrm{H} 5+\mathrm{H} 2 \mathrm{O} \\
870.00\end{array}$ & & \multicolumn{2}{|c|}{$3.540 \mathrm{E}+06$} & 2.120 \\
\hline $\begin{array}{l}\mathrm{OH}+\mathrm{CH} 2 \mathrm{CO}<=>\mathrm{HCCO}+\mathrm{H} 2 \mathrm{O} \\
2000.00\end{array}$ & & \multicolumn{2}{|c|}{$7.500 \mathrm{E}+12$} & .000 \\
\hline $\begin{array}{l}2 \mathrm{HO} 2<=>02+\mathrm{H} 2 \mathrm{O} 2 \\
1630.00\end{array}$ & & \multicolumn{2}{|c|}{$1.300 \mathrm{E}+11$} & .000 \\
\hline DUPLICATE & & & & \\
\hline $\begin{array}{l}2 \mathrm{HO} 2<=>02+\mathrm{H} 2 \mathrm{O} 2 \\
12000.00\end{array}$ & & \multicolumn{2}{|c|}{$4.200 \mathrm{E}+14$} & .000 \\
\hline $\begin{array}{l}\mathrm{HO} 2+\mathrm{CH} 2<=>\mathrm{OH}+\mathrm{CH} 2 \mathrm{O} \\
\mathrm{HO} 2+\mathrm{CH} 3<=>\mathrm{O} 2+\mathrm{CH} 4 \\
\mathrm{HO} 2+\mathrm{CH} 3<=>\mathrm{OH}+\mathrm{CH} 3 \mathrm{O}\end{array}$ & $\begin{array}{l}2.000 \mathrm{E}+13 \\
1.000 \mathrm{E}+12 \\
3.780 \mathrm{E}+13\end{array}$ & $\begin{array}{l}.000 \\
.000 \\
.000\end{array}$ & $\begin{array}{l}.00 \\
.00 \\
.00\end{array}$ & \\
\hline DUPLICATE & & & & \\
\hline $\mathrm{HO} 2+\mathrm{CO}<=>\mathrm{OH}+\mathrm{CO} 2$ & & $500 \mathrm{E}+14$ & .000 & 2360 \\
\hline
\end{tabular}




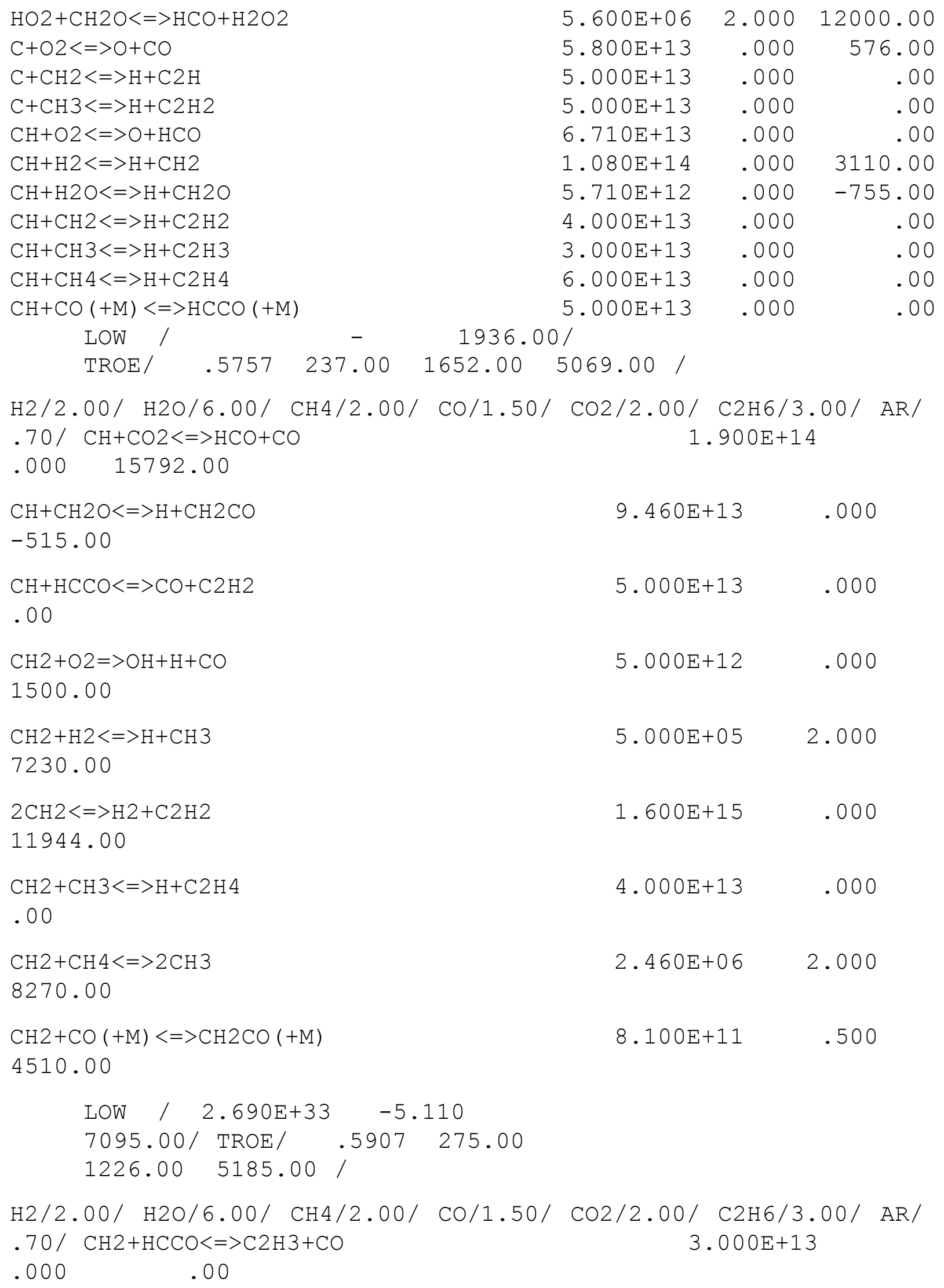




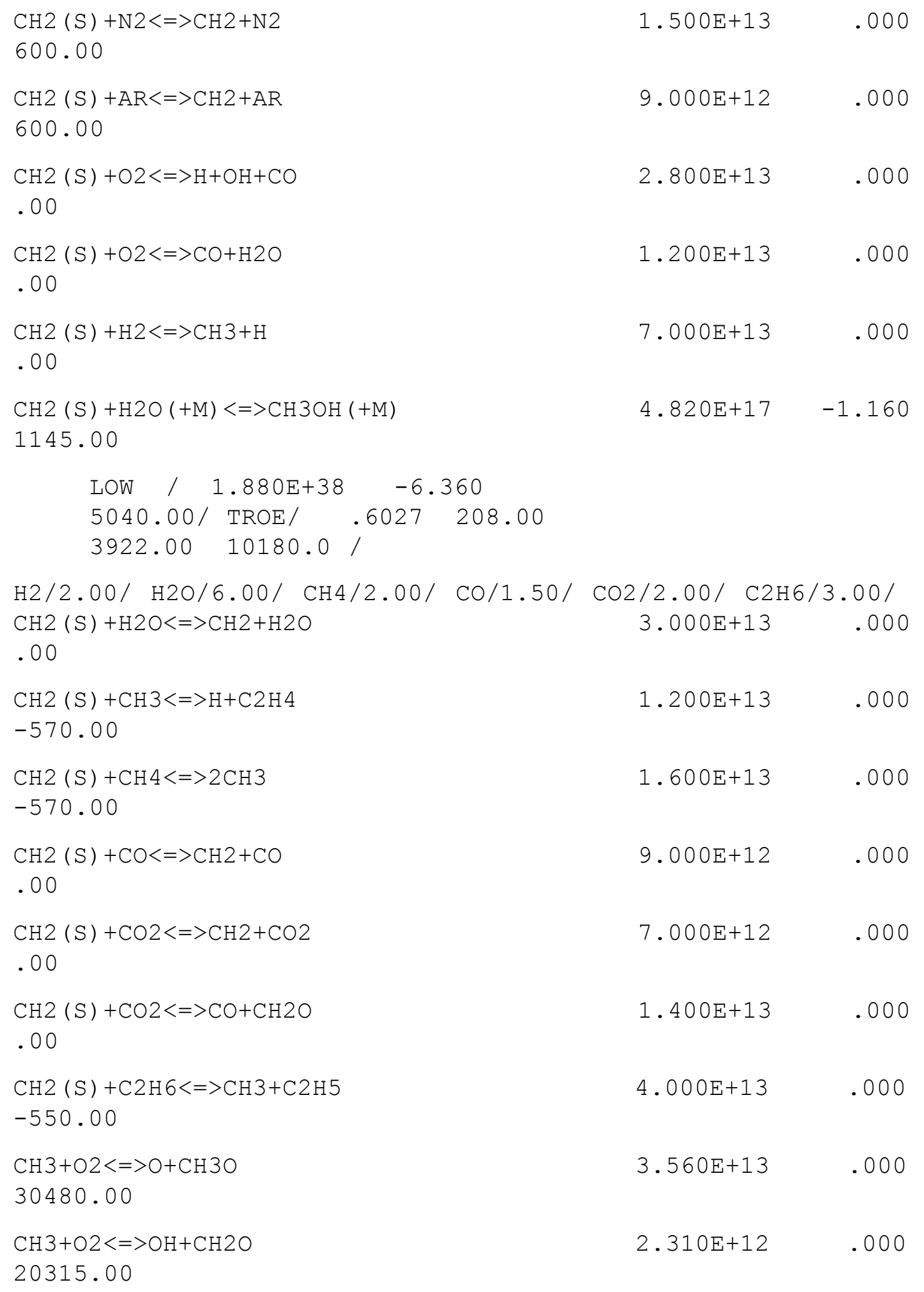




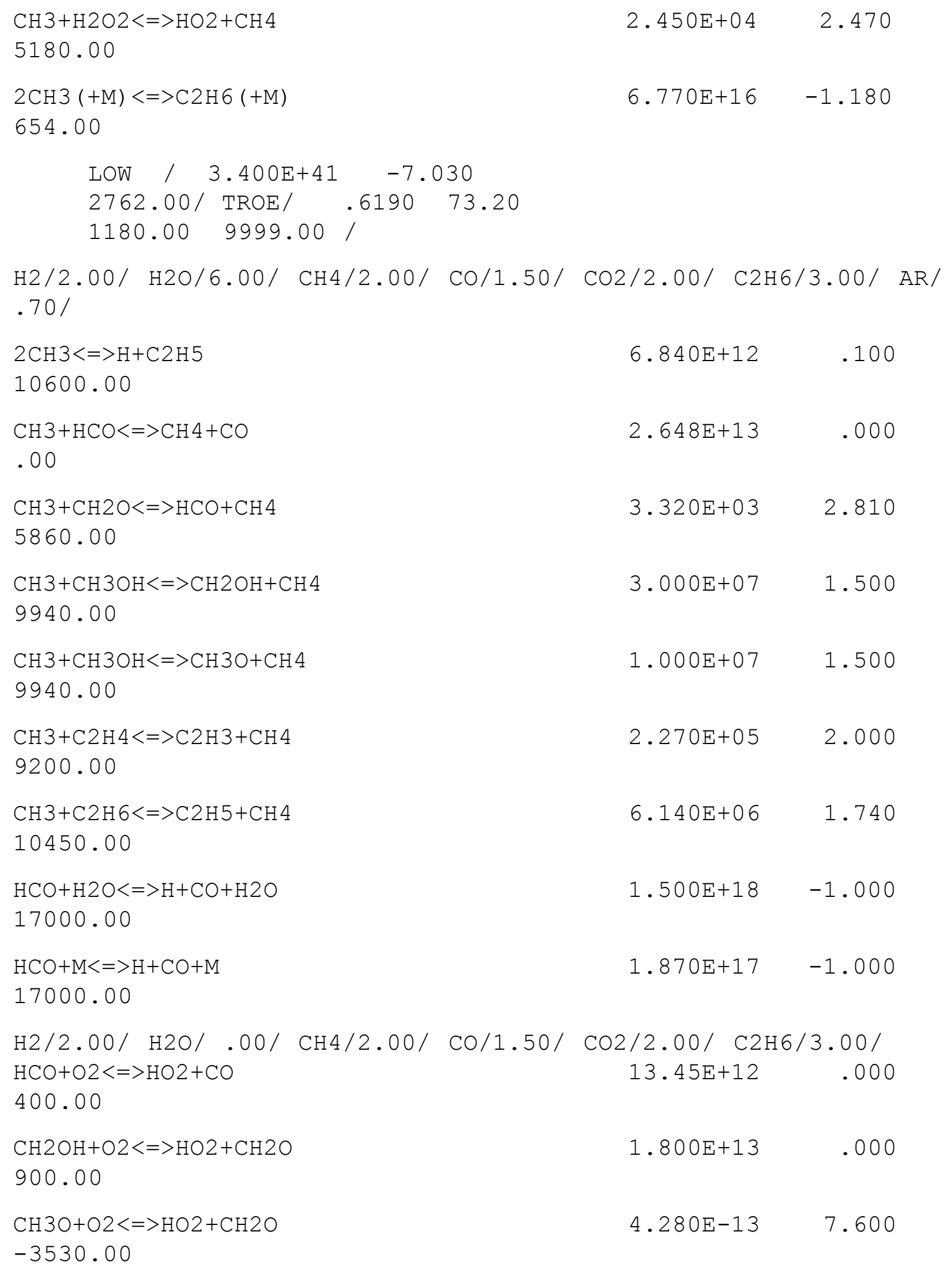




\begin{tabular}{|c|c|c|c|}
\hline $\begin{array}{l}\mathrm{C} 2 \mathrm{H}+\mathrm{O} 2<=>\mathrm{HCO}+\mathrm{CO} \\
-755.00\end{array}$ & \multicolumn{2}{|c|}{$1.000 \mathrm{E}+13$} & .000 \\
\hline $\begin{array}{l}\mathrm{C} 2 \mathrm{H}+\mathrm{H} 2<=>\mathrm{H}+\mathrm{C} 2 \mathrm{H} 2 \\
1993.00\end{array}$ & \multicolumn{2}{|c|}{$5.680 E+10$} & 0.900 \\
\hline $\begin{array}{l}\mathrm{C} 2 \mathrm{H} 3+\mathrm{O} 2<=>\mathrm{HCO}+\mathrm{CH} 2 \mathrm{O} \\
1015.00\end{array}$ & \multicolumn{2}{|c|}{$4.580 E+16$} & -1.390 \\
\hline $\begin{array}{l}\mathrm{C} 2 \mathrm{H} 4(+\mathrm{M})<=>\mathrm{H} 2+\mathrm{C} 2 \mathrm{H} 2(+\mathrm{M}) \\
86770.00\end{array}$ & \multicolumn{2}{|c|}{$8.000 \mathrm{E}+12$} & .440 \\
\hline $\begin{array}{l}\text { LOW / } 1.580 \mathrm{E}+51 \quad-9.300 \\
97800.00 / \mathrm{TROE} / \quad .7345 \quad 180.00 \\
1035.005417 .00 /\end{array}$ & & & \\
\hline $\mathrm{C} 2 \mathrm{H} 5+\mathrm{O} 2<=>\mathrm{HO} 2+\mathrm{C} 2 \mathrm{H} 4$ & $8.400 E+11$ & .000 & 3875.00 \\
\hline $\mathrm{HCCO}+\mathrm{O} 2<=>\mathrm{OH}+2 \mathrm{CO}$ & $3.200 E+12$ & .000 & 854.00 \\
\hline $2 \mathrm{HCCO}<=>2 \mathrm{CO}+\mathrm{C} 2 \mathrm{H} 2$ & $1.000 E+13$ & .000 & .00 \\
\hline $\mathrm{N}+\mathrm{NO}<=>\mathrm{N} 2+\mathrm{O}$ & $2.700 \mathrm{E}+13$ & .000 & 355.00 \\
\hline $\mathrm{N}+\mathrm{O} 2<=>\mathrm{NO}+\mathrm{O}$ & $9.000 E+09$ & 1.000 & 6500.00 \\
\hline $\mathrm{N}+\mathrm{OH}<=>\mathrm{NO}+\mathrm{H}$ & $3.360 E+13$ & .000 & 385.00 \\
\hline $\mathrm{N} 2 \mathrm{O}+\mathrm{O}<=>\mathrm{N} 2+\mathrm{O} 2$ & 1. $400 \mathrm{E}+12$ & .000 & 10810.00 \\
\hline $\mathrm{N} 2 \mathrm{O}+\mathrm{O}<=>2 \mathrm{NO}$ & $2.900 E+13$ & .000 & 23150.00 \\
\hline $\mathrm{N} 2 \mathrm{O}+\mathrm{H}<=>\mathrm{N} 2+\mathrm{OH}$ & $3.870 \mathrm{E}+14$ & .000 & 18880.00 \\
\hline $\mathrm{N} 2 \mathrm{O}+\mathrm{OH}<=>\mathrm{N} 2+\mathrm{HO} 2$ & $2.000 E+12$ & .000 & 21060.00 \\
\hline $\begin{array}{c}\mathrm{N} 2 \mathrm{O}(+\mathrm{M})<=>\mathrm{N} 2+\mathrm{O}(+\mathrm{M}) \\
\mathrm{LOW} / \quad 6.370 \mathrm{E}+14 \quad .000 \quad 56640.00\end{array}$ & $7.910 E+10$ & .000 & 56020.00 \\
\hline $\begin{array}{l}\mathrm{H} 2 / 2.00 / \mathrm{H} 2 \mathrm{O} / 6.00 / \mathrm{CH} 4 / 2.00 / \mathrm{CO} / 1.50 / \\
\mathrm{HO} 2+\mathrm{NO}<=>\mathrm{NO} 2+\mathrm{OH} \\
\mathrm{NO}+\mathrm{O}+\mathrm{M}<=>\mathrm{NO} 2+\mathrm{M}\end{array}$ & $\begin{array}{l}\mathrm{CO} 2 / 2.00 / \\
2.110 \mathrm{E}+12 \\
1.060 \mathrm{E}+20\end{array}$ & $\begin{array}{r}\mathrm{C} 2 \mathrm{H} 6 / 3 \\
.000 \\
-1.410\end{array}$ & $\begin{array}{r}.00 / \mathrm{AR} / \\
-480.00 \\
.00\end{array}$ \\
\hline $\begin{array}{l}\mathrm{H} 2 / 2.00 / \mathrm{H} 2 \mathrm{O} / 6.00 / \mathrm{CH} 4 / 2.00 / \mathrm{CO} / 1.50 / \\
\mathrm{NO} 2+\mathrm{O}<=>\mathrm{NO}+\mathrm{O} 2\end{array}$ & $\begin{array}{l}\mathrm{CO} 2 / 2.00 / \\
3.900 \mathrm{E}+12\end{array}$ & $\begin{array}{r}\mathrm{C} 2 \mathrm{H} 6 / 3 \\
.000\end{array}$ & $\begin{array}{l}.00 / \mathrm{AR} / \\
-240.00\end{array}$ \\
\hline $\mathrm{NO} 2+\mathrm{H}<=>\mathrm{NO}+\mathrm{OH}$ & $1.320 \mathrm{E}+14$ & .000 & 360.00 \\
\hline $\mathrm{NH}+\mathrm{O}<=>\mathrm{NO}+\mathrm{H}$ & $4.000 E+13$ & .000 & .00 \\
\hline $\mathrm{NH}+\mathrm{H}<=>\mathrm{N}+\mathrm{H} 2$ & $3.200 E+13$ & .000 & 330.00 \\
\hline $\mathrm{NH}+\mathrm{OH}<=>\mathrm{HNO}+\mathrm{H}$ & $2.000 \mathrm{E}+13$ & .000 & .00 \\
\hline $\mathrm{NH}+\mathrm{OH}<=>\mathrm{N}+\mathrm{H} 2 \mathrm{O}$ & $2.000 E+09$ & 1.200 & .00 \\
\hline $\mathrm{NH}+\mathrm{O} 2<=>\mathrm{HNO}+\mathrm{O}$ & $4.610 E+05$ & 2.000 & 6500.00 \\
\hline $\mathrm{NH}+\mathrm{O} 2<=>\mathrm{NO}+\mathrm{OH}$ & $1.280 E+06$ & 1.500 & 100.00 \\
\hline $\mathrm{NH}+\mathrm{N}<=>\mathrm{N} 2+\mathrm{H}$ & $1.500 \mathrm{E}+13$ & .000 & .00 \\
\hline $\mathrm{NH}+\mathrm{H} 2 \mathrm{O}<=>\mathrm{HNO}+\mathrm{H} 2$ & $2.000 E+13$ & .000 & 13850.00 \\
\hline $\mathrm{NH}+\mathrm{NO}<=>\mathrm{N} 2+\mathrm{OH}$ & $2.160 E+13$ & -.230 & .00 \\
\hline $\mathrm{NH}+\mathrm{NO}<=>\mathrm{N} 2 \mathrm{O}+\mathrm{H}$ & $3.650 \mathrm{E}+14$ & -.450 & .00 \\
\hline $\mathrm{NH} 2+\mathrm{O}<=>\mathrm{OH}+\mathrm{NH}$ & $3.000 \mathrm{E}+12$ & .000 & .00 \\
\hline
\end{tabular}


$\mathrm{H} 2 / 2.00 / \mathrm{H} 2 \mathrm{O} / 6.00 / \mathrm{CH} 4 / 2.00 / \mathrm{CO} / 1.50 / \mathrm{CO} 2 / 2.00 / \mathrm{C} 2 \mathrm{H} 6 / 3.00 / \mathrm{AR} /$ $.70 /$

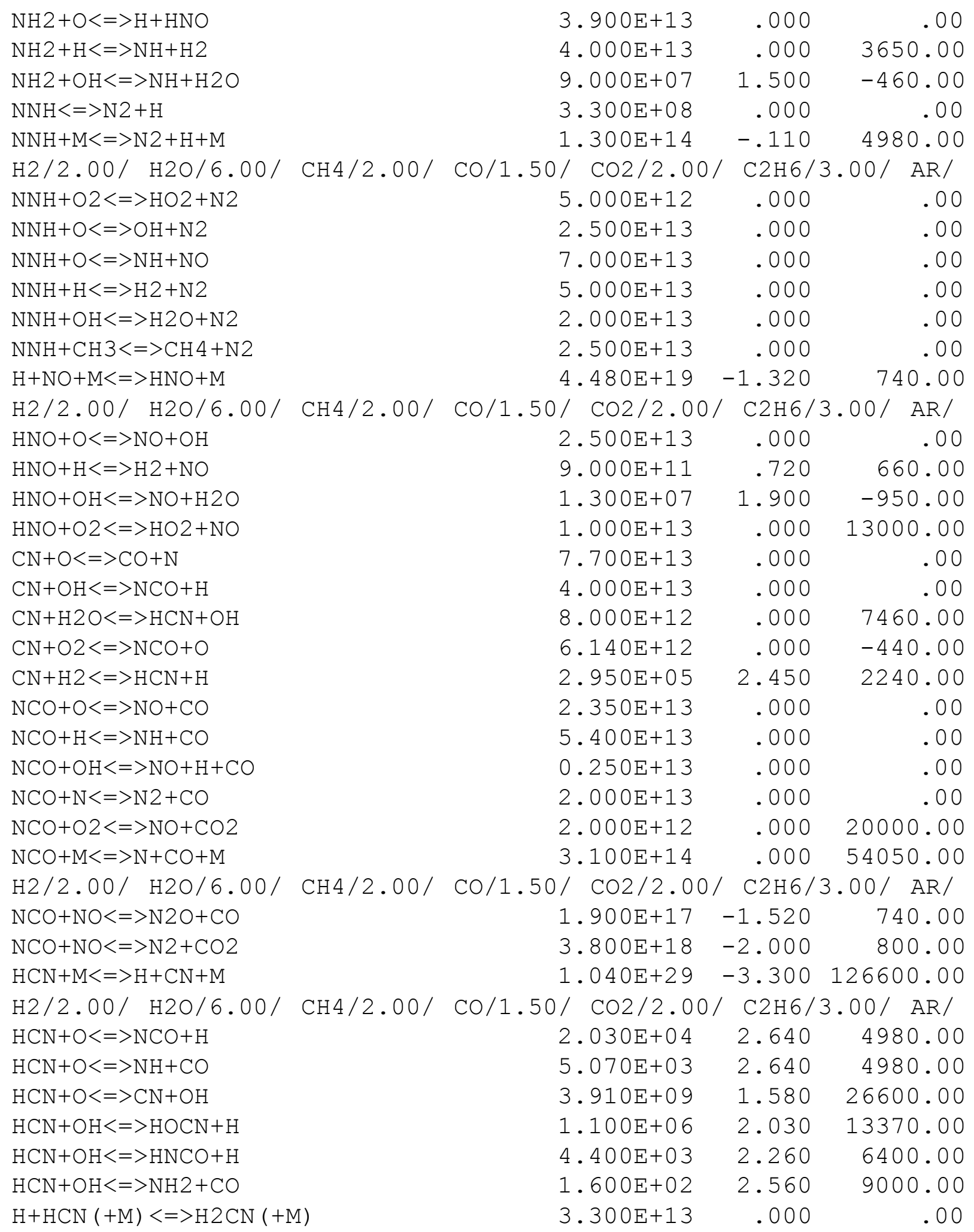


LOW /

$\mathrm{H} 2 / 2.00 / \mathrm{H} 2 \mathrm{O} / 6.00 /$ $\mathrm{H} 2 \mathrm{CN}+\mathrm{N}<=>\mathrm{N} 2+\mathrm{CH} 2$

$\mathrm{C}+\mathrm{N} 2<=>\mathrm{CN}+\mathrm{N}$

$\mathrm{CH}+\mathrm{N} 2<=>\mathrm{HCN}+\mathrm{N}$

$\mathrm{CH}+\mathrm{N} 2(+\mathrm{M})<=>\mathrm{HCNN}(+\mathrm{M})$

LOW /

TROE /

.6670
$-$

$\mathrm{CH} 4 / 2.00 /$

$1900.00 \%$

$\mathrm{CO} / 1.50 / \mathrm{CO} 2 / 2.00 /$

$6.000 \mathrm{E}+13$

$6.300 \mathrm{E}+13$

$3.120 \mathrm{E}+09$

3. $100 \mathrm{E}+12$

- $\quad 740.00 /$

$235.00-2117.00-4536.00 /$

$\mathrm{H} 2 / 2.00 / \mathrm{H} 2 \mathrm{O} / 6.00 / \mathrm{CH} 4 / 2.00 / \mathrm{CO} / 1.50 / \mathrm{CO} 2 / 2.00 / \mathrm{C} 2 \mathrm{H} 6 / 3.00 / \mathrm{AR} /$ $1.0 /$

$\mathrm{CH} 2+\mathrm{N} 2<=>\mathrm{HCN}+\mathrm{NH}$

$\mathrm{CH} 2(\mathrm{~S})+\mathrm{N} 2<=>\mathrm{NH}+\mathrm{HCN}$

$\mathrm{C}+\mathrm{NO}<=>\mathrm{CN}+\mathrm{O}$

$\mathrm{C}+\mathrm{NO}<=>\mathrm{CO}+\mathrm{N}$

$\mathrm{CH}+\mathrm{NO}<=>\mathrm{HCN}+\mathrm{O}$

$\mathrm{CH}+\mathrm{NO}<=>\mathrm{H}+\mathrm{NCO}$

$\mathrm{CH}+\mathrm{NO}<=>\mathrm{N}+\mathrm{HCO}$

$\mathrm{CH} 2+\mathrm{NO}<=>\mathrm{H}+\mathrm{HNCO}$

$\mathrm{CH} 2+\mathrm{NO}<=>\mathrm{OH}+\mathrm{HCN}$

$\mathrm{CH} 2+\mathrm{NO}<=>\mathrm{H}+\mathrm{HCNO}$

$\mathrm{CH} 2(\mathrm{~S})+\mathrm{NO}<=>\mathrm{H}+\mathrm{HNCO}$

$\mathrm{CH} 2(\mathrm{~S})+\mathrm{NO}<=>\mathrm{OH}+\mathrm{HCN}$

$\mathrm{CH} 2(\mathrm{~S})+\mathrm{NO}<=>\mathrm{H}+\mathrm{HCNO}$

$\mathrm{CH} 3+\mathrm{NO}<=>\mathrm{HCN}+\mathrm{H} 2 \mathrm{O}$

$\mathrm{CH} 3+\mathrm{NO}<=>\mathrm{H} 2 \mathrm{CN}+\mathrm{OH}$

$\mathrm{HCNN}+\mathrm{O}<=>\mathrm{CO}+\mathrm{H}+\mathrm{N} 2$

$\mathrm{HCNN}+\mathrm{O}<=>\mathrm{HCN}+\mathrm{NO}$

$\mathrm{HCNN}+\mathrm{O} 2<=>\mathrm{O}+\mathrm{HCO}+\mathrm{N} 2$

$\mathrm{HCNN}+\mathrm{OH}<=>\mathrm{H}+\mathrm{HCO}+\mathrm{N} 2$

$\mathrm{HCNN}+\mathrm{H}<=>\mathrm{CH} 2+\mathrm{N} 2$

$\mathrm{HNCO}+\mathrm{O}<=>\mathrm{NH}+\mathrm{CO} 2$

$\mathrm{HNCO}+\mathrm{O}<=>\mathrm{HNO}+\mathrm{CO}$

$\mathrm{HNCO}+\mathrm{O}<=>\mathrm{NCO}+\mathrm{OH}$

$\mathrm{HNCO}+\mathrm{H}<=>\mathrm{NH} 2+\mathrm{CO}$

$\mathrm{HNCO}+\mathrm{H}<=>\mathrm{H} 2+\mathrm{NCO}$

$\mathrm{HNCO}+\mathrm{OH}<=>\mathrm{NCO}+\mathrm{H} 2 \mathrm{O}$

$\mathrm{HNCO}+\mathrm{OH}<=>\mathrm{NH} 2+\mathrm{CO} 2$

$\mathrm{HNCO}+\mathrm{M}<=>\mathrm{NH}+\mathrm{CO}+\mathrm{M}$

$\mathrm{H} 2 / 2.00 / \mathrm{H} 2 \mathrm{O} / 6.00 /$

$\mathrm{HCNO}+\mathrm{H}<=>\mathrm{H}+\mathrm{HNCO}$

$\mathrm{HCNO}+\mathrm{H}<=>\mathrm{OH}+\mathrm{HCN}$

$\mathrm{HCNO}+\mathrm{H}<=>\mathrm{NH} 2+\mathrm{CO}$

$\mathrm{HOCN}+\mathrm{H}<=>\mathrm{H}+\mathrm{HNCO}$

$\begin{array}{lrr}1.000 \mathrm{E}+13 & .000 & 74000.00 \\ 1.000 \mathrm{E}+11 & .000 & 65000.00 \\ 1.900 \mathrm{E}+13 & .000 & .00 \\ 2.900 \mathrm{E}+13 & .000 & .00 \\ 4.100 \mathrm{E}+13 & .000 & .00 \\ 1.620 \mathrm{E}+13 & .000 & .00 \\ 2.460 \mathrm{E}+13 & .000 & .00 \\ 3.100 \mathrm{E}+17 & -1.380 & 1270.00 \\ 2.900 \mathrm{E}+14 & -.690 & 760.00 \\ 3.800 \mathrm{E}+13 & -.360 & 580.00 \\ 3.100 \mathrm{E}+17 & -1.380 & 1270.00 \\ 2.900 \mathrm{E}+14 & -.690 & 760.00 \\ 3.800 \mathrm{E}+13 & -.360 & 580.00 \\ 9.600 \mathrm{E}+13 & .000 & 28800.00 \\ 1.000 \mathrm{E}+12 & .000 & 21750.00 \\ 2.200 \mathrm{E}+13 & .000 & .00 \\ 2.000 \mathrm{E}+12 & .000 & .00 \\ 1.200 \mathrm{E}+13 & .000 & .00 \\ 1.200 \mathrm{E}+13 & .000 & .00 \\ 1.000 \mathrm{E}+14 & .000 & .00 \\ 9.800 \mathrm{E}+07 & 1.410 & 8500.00 \\ 1.500 \mathrm{E}+08 & 1.570 & 44000.00 \\ 2.200 \mathrm{E}+06 & 2.110 & 11400.00 \\ 2.250 \mathrm{E}+07 & 1.700 & 3800.00 \\ 1.050 \mathrm{E}+05 & 2.500 & 13300.00 \\ 3.300 \mathrm{E}+07 & 1.500 & 3600.00 \\ 3.300 \mathrm{E}+06 & 1.500 & 3600.00 \\ 1.180 \mathrm{E}+16 & .000 & 84720.00 \\ 1.50 / \mathrm{C} 02 / 2.00 / .02 \mathrm{H} / 3.00 / .8 R / \\ 2.100 \mathrm{E}+15 & -.690 & 2850.00 \\ 2.700 \mathrm{E}+11 & .180 & 2120.00 \\ 1.700 \mathrm{E}+14 & -.750 & 2890.00 \\ 2.000 \mathrm{E}+07 & 2.000 & 2000.00 \\ & & \\ 1.00 & \end{array}$




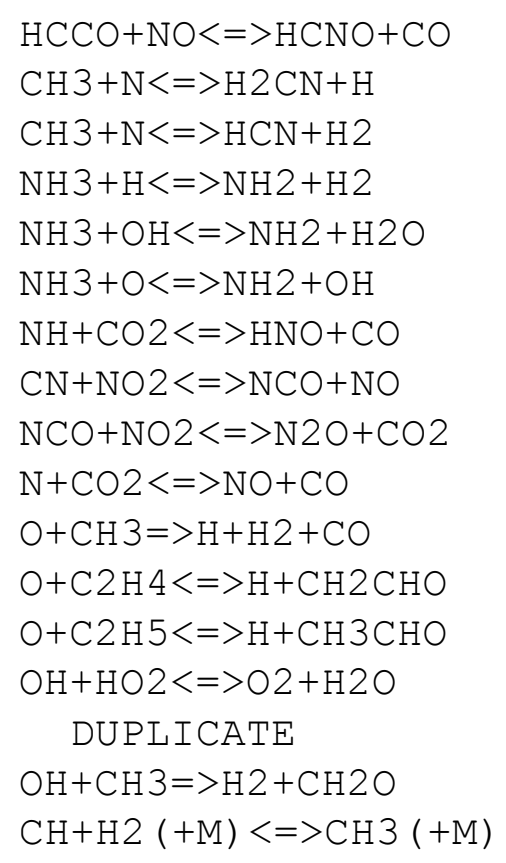

$\begin{array}{rrr}0.900 \mathrm{E}+13 & .000 & .00 \\ 6.100 \mathrm{E}+14 & -.310 & 290.00 \\ 3.700 \mathrm{E}+12 & .150 & -90.00 \\ 5.400 \mathrm{E}+05 & 2.400 & 9915.00 \\ 5.000 \mathrm{E}+07 & 1.600 & 955.00 \\ 9.400 \mathrm{E}+06 & 1.940 & 6460.00 \\ 1.000 \mathrm{E}+13 & .000 & 14350.00 \\ 6.160 \mathrm{E}+15 & -0.752 & 345.00 \\ 3.250 \mathrm{E}+12 & .000 & -705.00 \\ 3.000 \mathrm{E}+12 & .000 & 11300.00 \\ 3.370 \mathrm{E}+13 & .000 & .00 \\ 6.700 \mathrm{E}+06 & 1.830 & 220.00 \\ 1.096 \mathrm{E}+14 & .000 & .00 \\ 0.500 \mathrm{E}+16 & .000 & 17330.00 \\ 8.000 \mathrm{E}+09 & .500 & -1755.00 \\ 1.970 \mathrm{E}+12 & .430 & -370.00\end{array}$




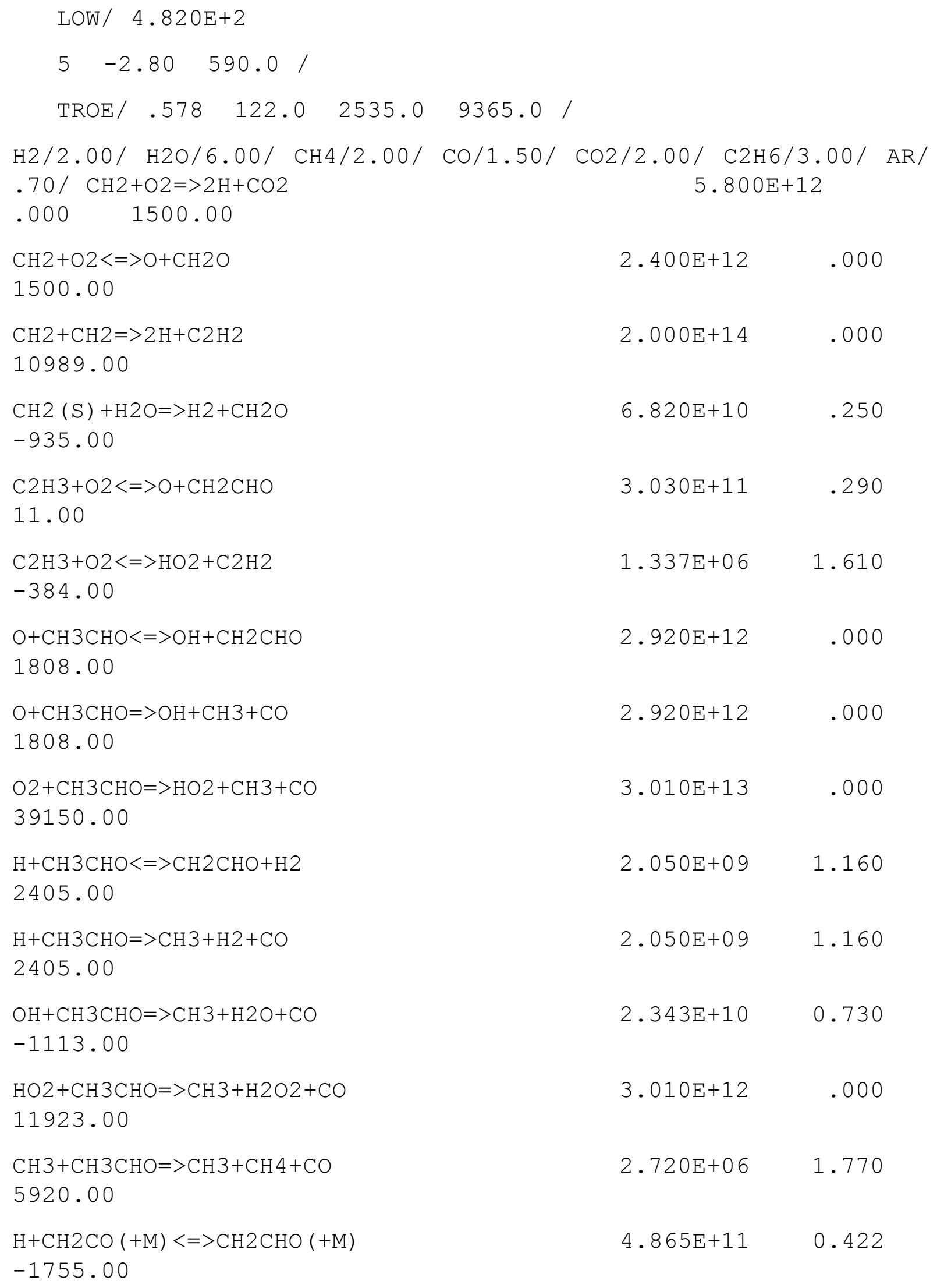




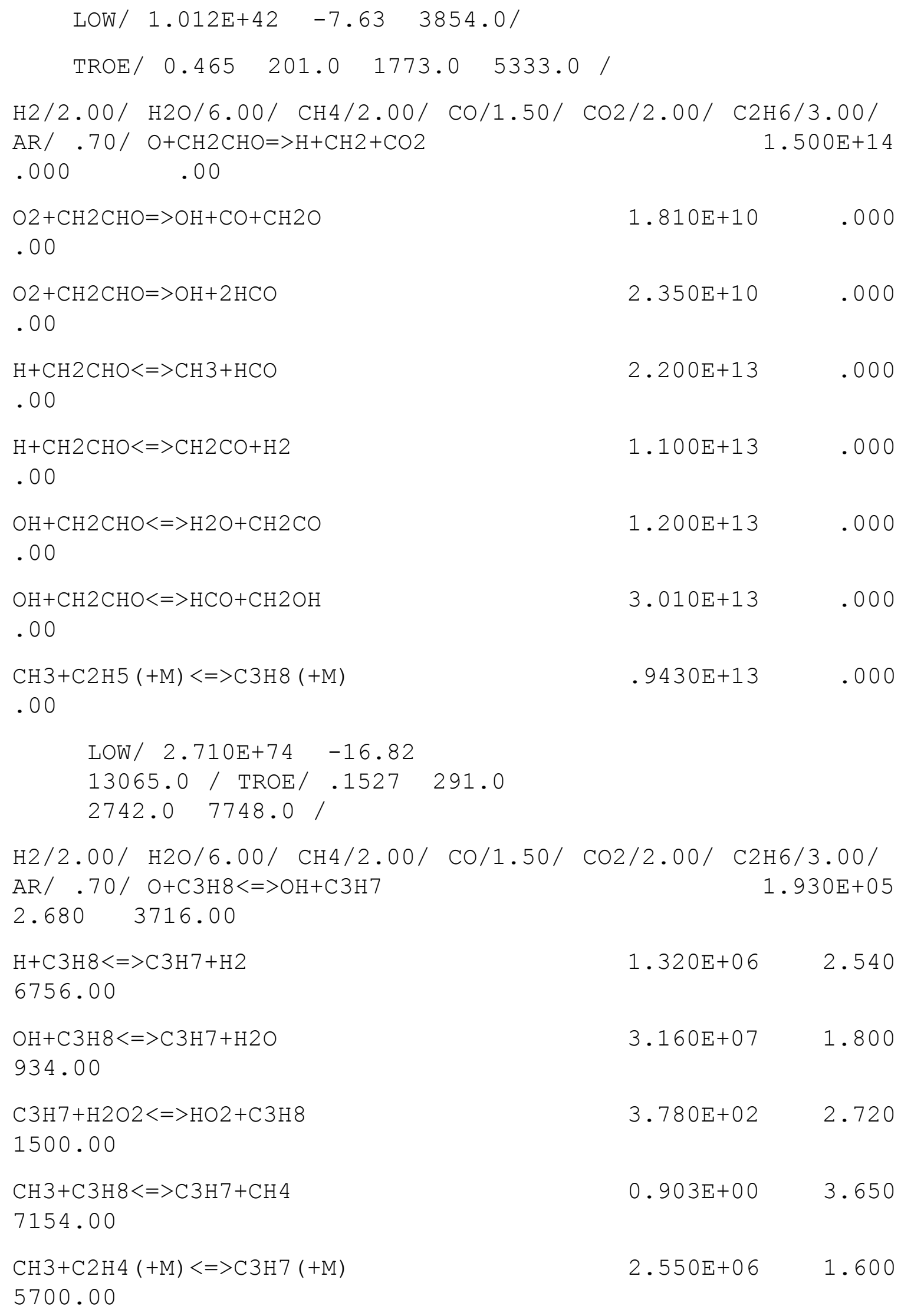




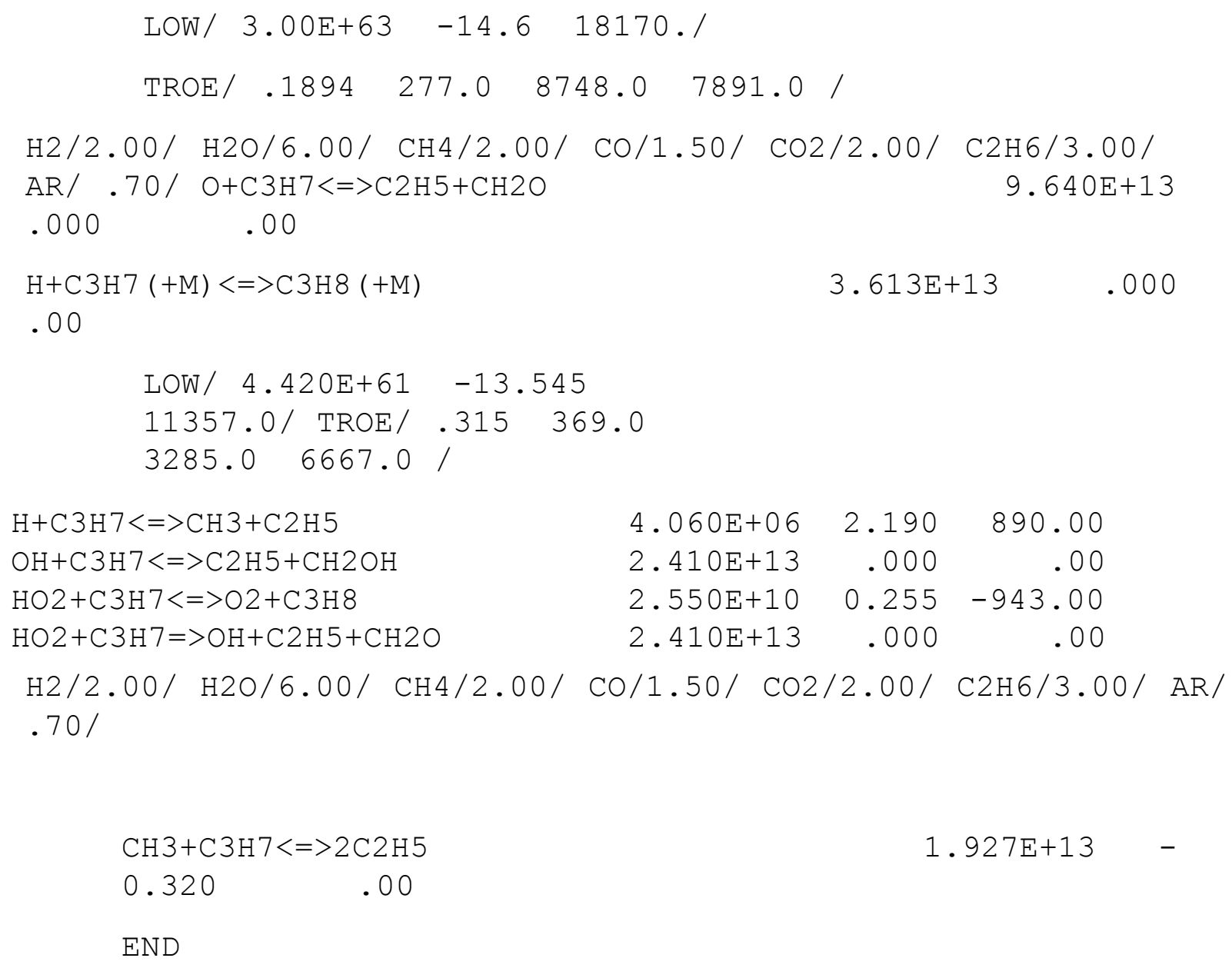

END 